Japan. J. Math.

Vol. 25, No. 1, 1999

\title{
Invariants des systèmes d'opérateurs différentiels et sommes formelles asymptotiques
}

\author{
By Jean Vaillant \\ (Received July 22, 1997) \\ (from Osaka Journal of Mathematics)
}

Table des Matières

Introduction

0. Enoncé des résultats concernant les conditions $\mathrm{L},(\mathrm{LG})_{d}$ et les réductions [22] [25]. Forme des sommes asymptotiques. Notations

§1. Étude du type (5) 16

$\S 2$. Étude du type $(4,1) \quad 48$

§3. Étude du type $(3,2) \quad 64$

$\S 4 . \quad$ Étude du type $(3,1,1) \quad 105$

$\S 5 . \quad$ Étude du type $(2,2,1) \quad 118$

$\S 6$. Étude du type $(2,1,1,1) \quad 142$

$\S 7$. Étude du type $(1,1,1,1) \quad 147$

§8. Applications 147

Références 151

\section{Introduction}

Nous avons présenté précédemment des conditions invariantes sur les systèmes et résumé leurs applications à la caractérisation des problèmes de Cauchy bien posés en $\mathcal{C}^{\infty}$ et dans les classes de Gevrey [26], [23], [24], [25]. Nous nous proposons de donner la rédaction détaillée de cette théorie. Un article concernera la définition des conditions $\mathrm{L},(\mathrm{LG})_{d}$, la réduction des systèmes et l'expression des conditions sur les systèmes réduits. Le présent article expose le coeur du problème. On précise les sommes formelles asymptotiques associées à chaque type de système et chaque cas défini par les conditions $\mathrm{L}$ ou $(\mathrm{LG})_{d}$. Ces développements permettent immédiatement la démonstration de la condition nécessaire pour que le problème de Cauchy soit bien posé en $\mathcal{C}^{\infty}$. Ils sont explicités pour la multiplicité 5 .

Ces sommes formelles aysmptotiques permettent de construire les paramétrixes en $\mathcal{C}^{\infty}$ et en Gevrey par prolongement des inégalités comme dans [11], [12], [13]. La 
nécessité en Gevrey est en préparation. Enfin un article montrera que les conditions $\mathrm{L}$ s'expriment aussi en terme de décomposition du diagonalisé de l'opérateur, et donnera l'exquisse de la preuve de la condition nécessaire et suffisante en $\mathcal{C}^{\infty}$ dans le cas général.

Dans un article [20] avec G. Taglialatela, nous avions par la méthode de changement d'ordre de W. Matsumoto [11], démontré que les conditions $\mathrm{L}$ sont nécessaires et suffisantes pour que le problème de Cauchy soit bien posé en $\mathcal{C}^{\infty}$. Les conditions L étaient explicitées microlocalement alors avec quelque restriction $\left(\mathbb{R}^{2}\right.$ au lieu de $\mathbb{R}^{n}$ ); elles sont ici données sous leur forme générale et des coefficients numériques sont précisés ou corrigés (conditions $\mathrm{L}_{4}, \mathrm{~L}_{5}$ du cas $(3,2)$ ). Nous avions donné une bibliographie assez complète alors [20] (cf. aussi [22]). Nous n'en reprendrons ici qu'une partie.

0. Enoncé des résultats concernant les conditions L, $(\mathbf{L G})_{d}$ et les réductions [22] [25]. Forme des sommes asymptotiques. Notations

0.1. $x \in \Omega, \Omega$ voisinage ouvert de 0 dans $\mathbb{R}^{n+1}$ (resp. $\mathbb{C}^{n+1}$ ); $h$ est un opérateur différentiel linéaire d'ordre $t$, matriciel $m \times m$ à coefficients analytiques. On note $a$ sa partie principale d'ordre $t$ et $b$ sa partie d'ordre $\leq t-1$, de sorte que: $h=a+b$.

On note $\xi$ la variable duale de $x$ et on considère le déterminant caractéristique: dét $a(x, \xi)$; on peut décomposer dét $a$ en facteurs irréductibles dans $\mathcal{O}[\xi]$, anneau des polynômes en $\xi$ à coefficients les germes de fonctions analytiques à l'origine; pour simplifier les notations, nous supposerons qu'il n'y a qu'un facteur multiple $H$, de multiplicité $m_{1}$, de sorte que:

$$
\text { dét } a=H^{m_{1}} K \text {. }
$$

Pour un opérateur différentiel ou pseudo-différentiel analytique classique (développable en symboles homogènes), matriciel ou scalaire $\Lambda^{\prime}(x, D)$ d'ordre $\leq \mu$, on notera: $\Lambda(x, \xi)=\sigma_{\mu}\left(\Lambda^{\prime}\right)$ le symbole d'ordre $\mu$ égal à la partie principale de $\Lambda^{\prime}$ si celle-ci est d'ordre $\mu$, à 0 sinon. Inversement à une matrice $\Lambda(x, \xi)$ de polynômes ou de symboles homogènes d'ordre $\mu$, on associera des opérateurs matriciels notés $\Lambda^{\prime}(x, D)$, de sorte que: $\sigma_{\mu}\left(\Lambda^{\prime}\right)=\Lambda$. On posera: $s=$ degré de $h, \chi=$ degré de $k$. Ainsi on notera: $H^{\prime}(x, D)$ tel que: $\sigma_{s}\left(H^{\prime}\right)=H I, I$ matrice unité de dimension $m$ et $K^{\prime}(x, D)$ tel que: $\sigma_{\mu}\left(K^{\prime}\right)=K$.

Nous nous proposons de définir des conditions sur l'opérateur $h$ qui expriment son comportement relatif à $H$.

On note $A$ la matrice des cofacteurs de $a$ de sorte que:

$$
a A=A a=\operatorname{dét} a I=H^{m_{1}} K I \text {. }
$$


On considère l'anneau localisé [22] de l'anneau $\mathcal{O}[\xi]$ par rapport à l'idéal premier défini par $H$; cet anneau est principal et dans cet anneau $a$ est équivalent à la matrice diagonale:

$$
\operatorname{diag}\left[H^{p}, H^{q_{1}}, \ldots, H^{q_{\ell}}, 1, \ldots, 1\right]
$$

où les entiers $p, q_{1}, \ldots, q_{\ell}$ sont tels que:

$$
p \geq q_{1} \geq \cdots \geq q_{\ell}>0 ; \quad p+q=m_{1},
$$

où l'on a posé: $q=q_{1}+\cdots+q_{\ell}$.

DÉfinition 1. On appelle type de l'opérateur la suite:

$$
\left(p, q_{1}, \ldots, q_{\ell}\right)
$$

A est divisible par $H^{q}$ dans $\mathcal{O}[\xi]$; on pose:

$$
\mathcal{A}=\frac{A}{H^{q}}
$$

de sorte que:

$$
a \mathcal{A}=\mathcal{A} a=H^{p} K I
$$

On note $\gamma=s+\chi-1, \mu_{0}=p s+\chi-1=$ degré de $\mathcal{A}+t-1$

$$
\begin{array}{r}
\mu_{j}=\mu_{0}+j \gamma+\left(\sum_{1 \leq k \leq j} q_{k}-j\right) s, \text { pour: } 0 \leq j \leq \ell, \\
\mu_{j}=\mu_{0}+j \gamma+(q-\ell) s, \text { pour: } \ell+1 \leq j .
\end{array}
$$

Définition 2. Conditions $\mathbf{L}$ - On les définit par récurrence.

Condition $\mathrm{L}_{1}$ : Il existe des opérateurs différentiels $\mathcal{A}^{\prime}, H^{\prime}, K^{\prime}$ et un polynôme $\Lambda_{1}(x, \xi)$ homogène en $\xi$ à coefficients matriciels de degré $\mu_{1}$, ou nul tels que:

$$
S_{0} \equiv \mathcal{A} \sigma_{\mu_{0}}\left(h \mathcal{A}^{\prime}-{H^{\prime}}^{p} K^{\prime}\right)=H^{p-q_{1}} \Lambda_{1}
$$

On a alors:

$$
a \Lambda_{1}=H^{q_{1}} K \sigma_{\mu_{0}}\left(h \mathcal{A}^{\prime}-H^{\prime p} K^{\prime}\right) .
$$

On suppose $\mathrm{L}_{1}$ réalisée, $\mathcal{A}^{\prime}, H^{\prime}, K^{\prime}$ et $\Lambda_{1}$ choisis. 
Condition $\mathrm{L}_{2}$ : Il existe un opérateur différentiel $\Lambda_{1}^{\prime}$ et un polynôme $\Lambda_{2}$ homogène en $\xi$ de degré $\mu_{2}$ ou nul, tels que:

$$
S_{1} \equiv \mathcal{A} \sigma_{\mu_{1}}\left(h \Lambda_{1}^{\prime}-h \mathcal{A}^{\prime} H^{\prime q_{1}} K^{\prime}+H^{\prime p} K^{\prime} H^{\prime q_{1}} K^{\prime}\right)=H^{p-q_{2}} \Lambda_{2}
$$

Condition $\mathrm{L}_{\ell}$ : Il existe un opérateur différentiel $\Lambda_{\ell-1}^{\prime}$ et un polynôme $\Lambda_{\ell}$ tels que:

$$
\begin{aligned}
S_{\ell-1} \equiv & \mathcal{A} \sigma_{\mu_{\ell-1}}\left(h \Lambda_{\ell-1}^{\prime}-h \Lambda_{\ell-2}^{\prime} H^{\prime q_{\ell}-1} K^{\prime}+\cdots\right. \\
& +(-1)^{\ell-1} h \mathcal{A}^{\prime} H^{\prime q_{1}} K^{\prime} H^{\prime q_{2}} K^{\prime} \ldots H^{\prime q_{\ell-1}} K^{\prime} \\
& \left.+(-1)^{\ell} H^{\prime p} K^{\prime} H^{\prime q_{1}} K^{\prime} \ldots H^{\prime q_{\ell}-1} K^{\prime}\right)=H^{p-q_{\ell}} \Lambda_{\ell} .
\end{aligned}
$$

Condition $\mathrm{L}_{\ell+1}$ : Il existe $\Lambda_{\ell}^{\prime}$ et $\Lambda_{\ell+1}$ tels que:

$$
\begin{aligned}
S_{\ell} \equiv & \mathcal{A} \sigma_{\mu_{\ell}}\left(h \Lambda_{\ell}^{\prime}-h \Lambda_{\ell-1}^{\prime} H^{\prime q_{\ell}} K^{\prime}+\cdots+(-1)^{\ell} h \mathcal{A}^{\prime} H^{\prime q_{1}} K^{\prime} \ldots H^{\prime q_{\ell}} K^{\prime}\right. \\
& \left.+(-1)^{\ell+1} H^{\prime p} K^{\prime} . H^{\prime q_{1}} K^{\prime} \ldots H^{\prime q_{\ell}} K^{\prime}\right)=H^{p-1} \Lambda_{\ell+1} .
\end{aligned}
$$

Condition $\mathrm{L}_{m_{1}^{\prime}}$ : Il existe $\Lambda_{m_{1}^{\prime}-1}^{\prime}$ et $\Lambda_{m_{1}^{\prime}}$ tels que:

$$
\begin{aligned}
S_{m_{1}^{\prime}-1} \equiv & \mathcal{A} \sigma_{\mu_{m_{1}^{\prime}-1}}\left(h \Lambda_{m_{1}^{\prime}-1}^{\prime}-h \Lambda_{m_{1}^{\prime}-2}^{\prime} H^{\prime} K^{\prime}+\cdots\right. \\
& +(-1)^{m_{1}^{\prime}-1} h \mathcal{A}^{\prime} H^{\prime q_{1}} K^{\prime} \ldots H^{\prime q_{\ell}} K^{\prime}\left(H^{\prime} K^{\prime}\right)^{m_{1}-\ell-1} \\
& \left.+(-1)^{m_{1}^{\prime}} H^{\prime p} K^{\prime} H^{\prime q_{1}} K^{\prime} \ldots H^{\prime q_{\ell}} K^{\prime}\left(H^{\prime} K\right)^{m_{1}-\ell-1}\right) \\
= & H^{p-1} \Lambda_{m_{1}^{\prime}}^{\prime} .
\end{aligned}
$$

DÉFINITION 3. $m_{1}^{\prime}$ est le plus petit entier tel que toutes les conditions $\mathrm{L}_{m_{1}^{\prime \prime}}$, $m_{1}^{\prime \prime}>m_{1}^{\prime}$, soient des conséquences de $\mathrm{L}_{1}, \ldots, \mathrm{L}_{m_{1}^{\prime}}$.

REMARQUE. Les conditions L sont évidemment invariantes.

Proposition 1. Les conditions $\mathrm{L}$ ne dépendent pas du choix des opérateurs $H^{\prime}, K^{\prime}, \mathcal{A}^{\prime}, \ldots, \Lambda_{m_{1}^{\prime}-1}^{\prime}$.

ConsÉquence. On peut les écrire explicitement à l'aide des coefficients de $h$.

On étudiera désormais le cas hyperbolique. $h$ est donc un opérateur différentiel dans $\Omega \subset \mathbb{R}^{n+1}$; on suppose que $H K$ est strictement hyperbolique par rapport à

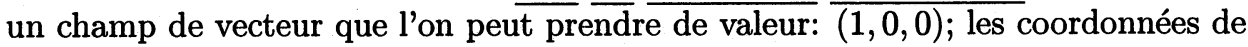
$x$ sont:

$$
x=\left(x_{0}, x^{\prime}\right)=\left(x_{0}, x_{1}, \ldots, x_{n}\right)
$$


$\xi=\left(\xi_{0}, \xi^{\prime}\right)=\left(\xi_{0}, \xi_{1}, \ldots, \xi_{n}\right) ;$ l'hyperbolicité exprime que $H K\left(x, \xi_{0}, \xi^{\prime}\right)=0$ a $s+\chi$ zéros réels distincts en $\xi_{0}, \forall x, \xi^{\prime} \neq 0$; les hyperplans $x_{0}=\underline{x}_{0}$ ne sont caractéristiques en aucun point; $h$ est donc hyperbolique à multiplicité constante; on suppose aussi pour simplifier que $h$ est de degré 1 .

Dans chaque cas de multiplicité 5 , on calculera $m_{1}^{\prime}$. Les conditions $\mathrm{L}$ s'expriment alors par l'annulation de $N\left(m_{1}^{\prime}\right)$ germes à l'origine de fonctions analytiques.

DÉfinition 4. On note $\mathcal{N}=\mathcal{N}\left(m_{1}, p, \ell\right)$ l'ensemble des points $\left(n_{1}, n_{2}\right)$ de coordonnées entières du quart de plan $\left(\mathbb{R}^{+}\right)^{2}$, tels que:

$$
0<n_{1} \leq m_{1} ; 0<n_{2} \leq r ; p n_{2} \leq(p-1) n_{1} \text {. }
$$

On a posé: $r=m_{1}-\ell-1$;

On note $c=c\left(m_{1}, p, \ell\right)$ le nombre de ces points.

Proposition 2. Pour $m_{1} \leq 5, N\left(m_{1}^{\prime}\right)=c$.

Remarque. Pour $m_{1}$ quelconque, l'évaluation: $N\left(m_{1}^{\prime}\right) \leq c$, généralise la précédente; elle sera expliquée dans une autre publication [27].

0.2. Pour l'étude du cas Gevrey, les conditions $L$ doivent être détaillées dans le sens suivant: on a vu qu'elles expriment l'annulation de $N\left(m_{1}^{\prime}\right)$ germes de fonctions analytiques à l'origine. Nous allons donner dans chaque cas: $m_{1}=5$, les conditions invariantes qui expriment l'annulation de chacun de ces germes ou de plusieurs d'entre eux; chaque condition $\mathrm{L}_{k}$ sera la réunion de telles conditions.

Définition 5. 1) Type (5). $\mathrm{L}_{1}$ s'écrit:

$$
S_{0}=H^{4} \Lambda_{1}
$$

On définit les conditions partielles:

$$
\mathrm{L}_{11}: S_{0}=H \Lambda_{11} ; \quad \mathrm{L}_{12}: S_{0}=H^{2} \Lambda_{12} ; \quad \mathrm{L}_{13}: S_{0}=H^{3} \Lambda_{13}
$$

on a alors à partir de $\mathrm{L}_{13}$ :

$$
H^{2} K \sigma_{\mu_{0}}\left(h A^{\prime}-H^{\prime 5} K^{\prime}\right)=a \Lambda_{13}
$$

on pose: $\mu_{(1)}=\mu_{1}+s$

$$
S_{(1)}=A \sigma_{\mu_{(1)}}\left[h \Lambda_{13}^{\prime}-h A^{\prime}{H^{\prime}}^{2} K^{\prime}+{H^{\prime}}^{5} K^{\prime}{H^{\prime}}^{2} K^{\prime}\right]
$$

$\mathrm{L}_{11}, \mathrm{~L}_{12}, \mathrm{~L}_{13}$ entraînent que $S_{(1)}$ est divisible par $H^{2} \cdot \mathrm{L}_{(2) 3}$ exprime que: $S_{(1)}=$ $H^{3} \Lambda_{(2) 3}$. L $\mathrm{L}_{14}$ s'écrit: $S_{0}=H^{4} \Lambda_{1}$, soit $\mathrm{L}_{1}$. 
L L $_{2}$ 'écrit: $S_{1}=H^{4} \Lambda_{2} ; \mathrm{L}_{1}$ entraîne que $S_{1}$ est divisible par $H$; la condition $\mathrm{L}_{22}: S_{1}=H^{2} \Lambda_{22}$ est équivalente à $\mathrm{L}_{(2) 3}$; la condition $\mathrm{L}_{23}$ s'écrit: $S_{1}=H^{3} \Lambda_{23}$ on en déduit:

$$
a \Lambda_{23}=H^{2} K \sigma_{\mu_{1}} h\left[\Lambda_{1}^{\prime}-h A^{\prime} H^{\prime} K^{\prime}+H^{\prime 5} K^{\prime} H^{\prime} K^{\prime}\right]
$$

On définit $\mu_{(2)}=\mu_{2}+s$

$$
S_{(2)}=A \sigma_{\mu_{(2)}}\left[h \Lambda_{23}^{\prime}-h \Lambda_{1}^{\prime}{H^{\prime}}^{2} K^{\prime}+h A^{\prime} H^{\prime} K^{\prime}{H^{\prime}}^{2} K^{\prime}-{H^{\prime}}^{5} K^{\prime} H^{\prime} K^{\prime}{H^{\prime}}^{2} K^{\prime}\right],
$$

la divisibilité de $S_{(2)}$ par $H^{3}$ est impliqué par les conditions précédentes. On note $\mathrm{L}_{(3) 4}$ la condition: $S_{(2)}=H^{4} \Lambda_{(3) 4} . \mathrm{L}_{24}$ s'écrit $S_{1}=H^{4} \Lambda_{2}$ soit $\mathrm{L}_{2}$.

$\mathrm{L}_{3}$ s'écrit: $S_{2}=H^{4} \Lambda_{3} ; \mathrm{L}_{1}, \mathrm{~L}_{2}$ entraînent que $S_{2}$ est divisible par $H^{2}$; la condition $\mathrm{L}_{33}: S_{2}=H^{3} \Lambda_{3,3}$ est équivalente à: $\mathrm{L}_{(3) 4}$.

$\mathrm{L}_{34}$ s'écrit: $S_{2}=H^{4} \Lambda_{3}$ soit $\mathrm{L}_{3}$.

$\mathrm{L}_{4}$ s'écrit: $S_{4}=H^{4} \Lambda_{3} ; \mathrm{L}_{1}, \mathrm{~L}_{2}, \mathrm{~L}_{3}$ impliquent la divisibilité de $S_{4}$ par $H^{3}$; $\mathrm{L}_{44}: S_{4}=H^{4} \Lambda_{3}$ est $\mathrm{L}_{4}$.

2) Type (4,1). $\mathrm{L}_{1}$ s'écrit: $S_{0}=H^{3} \Lambda_{1}$. On définit les conditions partielles:

$$
\mathrm{L}_{11}: S_{0}=H \Lambda_{11}, \quad \mathrm{~L}_{12}: S_{0}=H^{2} \Lambda_{12}
$$

si $\mathrm{L}_{12}$ est vérifiée, on a:

$$
a \Lambda_{12}=H^{2} K \sigma_{\mu_{0}}\left[h \mathcal{A}^{\prime}-H^{\prime 4} K^{\prime}\right]
$$

on définit $\mathrm{L}_{(2) 2}$ :

$$
\mathcal{A} \sigma_{\mu_{(1)}}\left[h \Lambda_{12}^{\prime}-h \mathcal{A}^{\prime}{H^{\prime}}^{2} K^{\prime}+{H^{\prime}}^{4} K^{\prime}{H^{\prime}}^{2} K^{\prime}\right]=H^{2} \Lambda_{(2) 2}
$$

$\mu_{(1)}=\mu_{1}+s$. La divisibilité par $H$ est une conséquence de $\mathrm{L}_{12} ; \mathrm{L}_{13}$ est la condition $\mathrm{L}_{1}$.

$\mathrm{L}_{2}$ s'écrit: $S_{1}=H^{3} \Lambda_{2}$. On définit les conditions:

$\mathrm{L}_{21}: S_{1}=H \Lambda_{21} ;$ si $\mathrm{L}_{1}$ est vérifiée, $\mathrm{L}_{21}$ équivaut à $\mathrm{L}_{(2) 2} . \mathrm{L}_{22}: S_{1}=H^{2} \Lambda_{2,2} ;$ si $\mathrm{L}_{22}$ est vérifiée, on a:

$$
a \Lambda_{2,2}=H^{2} K \sigma_{\mu_{1}}\left[h \Lambda_{1}^{\prime}-h \mathcal{A}^{\prime} H^{\prime} K^{\prime}+H^{\prime 4} K^{\prime} H^{\prime} K^{\prime}\right]
$$

on définit:

$$
\begin{aligned}
\mathrm{L}_{(3) 3}: \mathcal{A} \sigma_{\mu_{(1)}}\left[h \Lambda_{22}^{\prime}-h \Lambda_{1}^{\prime}{H^{\prime}}^{2} K^{\prime}+\right. & h \mathcal{A}^{\prime} H^{\prime} K^{\prime}{H^{\prime}}^{2} K^{\prime} \\
& \left.-H^{\prime}{ }^{4} K^{\prime} H^{\prime} K^{\prime} H^{\prime 2} K^{\prime}\right]=H^{3} \Lambda_{(3) 3}
\end{aligned}
$$


$\mu_{(2)}=\mu_{2}+s$. La divisibilité par $H^{2}$ est une conséquence de $\mathrm{L}_{22}$.

$\mathrm{L}_{23}$ est la condition $\mathrm{L}_{2}$.

$\mathrm{L}_{3}$ s'écrit: $S_{2}=H^{3} \Lambda_{3}$; la divisibilité par $H$ est une conséquence de $\mathrm{L}_{2}$;

$\mathrm{L}_{32}: S_{2}=H^{2} \Lambda_{32}$ équivaut à $\mathrm{L}_{(3) 3} . \mathrm{L}_{33}$ est $\mathrm{L}_{3}$.

$\mathrm{L}_{4}$ s'écrit: $S_{3}=H^{3} \Lambda_{4}$. Si $\mathrm{L}_{3}$ est vérifiée, la condition $\mathrm{L}_{42}: S_{3}=H^{2} \Lambda_{42}$ est vérifiée. Il reste donc:

$$
\mathrm{L}_{4} \equiv \mathrm{L}_{43}
$$

qui exprime la divisibilité de $S_{3}$ par $H^{3}$.

3) Type $(3,2) . \quad \mathrm{L}_{1}$ s'écrit: $S_{0}=H \Lambda_{1}$.

On définit la condition: $\mathrm{L}_{12}: S_{0}=H^{2} \Lambda_{12}$.

$\mathrm{L}_{2}$ s'écrit: $S_{1}=H^{2} \Lambda_{2}$, on définit: $\mathrm{L}_{21}: S_{1}=H \Lambda_{21}$.

La condition $\mathrm{L}_{22}: S_{1}=H^{2} \Lambda_{2}$ est la condition $\mathrm{L}_{2} ; \mathrm{L}_{12}$ implique $\mathrm{L}_{1}$ et $\mathrm{L}_{2}$.

$\mathrm{L}_{3}$ s'écrit: $S_{2}: H^{2} \Lambda_{3}$. On définit: $\mathrm{L}_{31}: S_{2}=H \Lambda_{31} ; \mathrm{L}_{31}$ est une conséquence de $\mathrm{L}_{1}, \mathrm{~L}_{2}$; on a:

$$
a \Lambda_{31}=H^{2} K \sigma_{\mu_{2}}\left[h \Lambda_{2}^{\prime}-h \Lambda_{1}^{\prime} H^{\prime} K^{\prime}+h \mathcal{A}^{\prime}{H^{\prime}}^{2} K^{\prime} H^{\prime} K^{\prime}-{H^{\prime}}^{3} K^{\prime}{H^{\prime}}^{2} K^{\prime} H^{\prime} K^{\prime}\right]
$$

on définit: $\mu_{(3)}=\mu_{3}+s$

$$
\begin{aligned}
S_{(3)}= & \mathcal{A} \sigma_{\mu_{(3)}}\left(h \Lambda_{31}^{\prime}-h \Lambda_{2}^{\prime}{H^{\prime}}^{2} K^{\prime}+h \Lambda_{1}^{\prime} H^{\prime} K^{\prime} H^{\prime 2} K^{\prime}\right. \\
& \left.-h \mathcal{A}^{\prime} H^{\prime 2} K^{\prime} H^{\prime} K^{\prime} H^{\prime 2} K^{\prime}+H^{\prime 3} K^{\prime} H^{\prime 2} K^{\prime} H^{\prime} K^{\prime} H^{\prime} K^{\prime}\right) .
\end{aligned}
$$

$S_{(3)}$ est divisible par $H$ si $\mathrm{L}_{1}, \mathrm{~L}_{2}$ sont vérifiées.

$\mathrm{L}_{(4) 2}$ exprime que: $S_{(3)}$ est divisible par $H^{2}$.

$\mathrm{L}_{32}: S_{2}=H^{2} \Lambda_{3}$ est la condition $\mathrm{L}_{3}$; si $\mathrm{L}_{12}$ est vérifiée, $\mathrm{L}_{3}$ implique $\mathrm{L}_{(4) 2}$.

$\mathrm{L}_{4}$ s'écrit: $S_{3}=H^{2} \Lambda_{4}$; on définit: $\mathrm{L}_{41}: S_{3}=H \Lambda_{41}$, si $\mathrm{L}_{1}, \mathrm{~L}_{2}, \mathrm{~L}_{3}$ sont vérifiées, $\mathrm{L}_{41}$ est vérifiée.

$\mathrm{L}_{5}$ s'écrit $S_{4}=H^{2} \Lambda_{5}$; la divisibilité par $H$ est impliqué par $\mathrm{L}_{1}, \mathrm{~L}_{2}, \mathrm{~L}_{3}, \mathrm{~L}_{4}$.

Si $\mathrm{L}_{12}, \mathrm{~L}_{3}, \mathrm{~L}_{4}$ sont satisfaites, $\mathrm{L}_{5}$ est satisfaite.

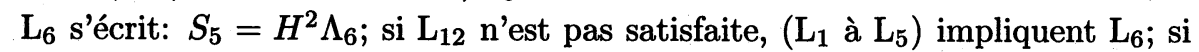
$\mathrm{L}_{12}$ est satisfaite, la divisibilité par $H$ est impliqué par $\mathrm{L}_{1}, \mathrm{~L}_{2}, \mathrm{~L}_{3}, \mathrm{~L}_{4}, \mathrm{~L}_{5}$.

$\mathrm{L}_{7}$ s'écrit: $S_{6}=H^{2} \Lambda_{7}$; si $\mathrm{L}_{12}$ n'est pas satisfaite $\left(\mathrm{L}_{1}\right.$ à $\left.\mathrm{L}_{5}\right)$ impliquent $\mathrm{L}_{7}$; si $\mathrm{L}_{12}, \mathrm{~L}_{3}, \mathrm{~L}_{4}, \mathrm{~L}_{6}$ sont satisfaites, $\mathrm{L}_{7}$ est satisfaite.

$\mathrm{L}_{8}$ s'écrit: $S_{7}=H^{2} \Lambda_{8} ;$ si $\mathrm{L}_{12}$ n'est pas satisfaite $\left(\mathrm{L}_{1}\right.$ à $\left.\mathrm{L}_{5}\right)$ impliquent $\mathrm{L}_{8}$; si $\mathrm{L}_{12}, \mathrm{~L}_{3}, \mathrm{~L}_{4}, \mathrm{~L}_{6}$ sont vérifiées, $S_{7}$ est divisible par $H$.

4) Type $(3,1,1) . \quad \mathrm{L}_{1}$ s'écrit: $S_{0}=H^{2} \Lambda_{1}$; on définit $\mathrm{L}_{11}: S_{0}=H \Lambda_{11} ; \mathrm{L}_{12}$ est alors évidemment $\mathrm{L}_{1}$.

$\mathrm{L}_{2}$ s'écrit: $S_{1}=H^{2} \Lambda_{2}$; on définit $\mathrm{L}_{21}: S_{1}=H \Lambda_{21} ;$ si $\mathrm{L}_{21}$ est vérifiée, on a:

$$
a \Lambda_{21}=H^{2} K \sigma_{\mu_{1}}\left[h \Lambda_{1}^{\prime}-h \mathcal{A}^{\prime} H^{\prime} K^{\prime}+{H^{\prime}}^{3} K^{\prime} H^{\prime} K^{\prime}\right]
$$


on définit: $\mu_{(2)}=\mu_{2}+s$

$$
\begin{aligned}
\mathrm{L}_{(3) 2}: \mathcal{A} \sigma_{\mu_{(2)}} h\left[\Lambda_{21}^{\prime}-h \Lambda_{1}^{\prime}{H^{\prime}}^{2} K^{\prime}+\right. & h \mathcal{A}^{\prime} H^{\prime} K^{\prime}{H^{\prime}}^{2} K^{\prime} \\
& \left.-H^{\prime 3} K^{\prime} H^{\prime} K^{\prime} H^{\prime 2} K^{\prime}\right]=H^{2} \Lambda_{32}
\end{aligned}
$$

la divisibilité par $H$ est une conséquence de $\mathrm{L}_{1}, \mathrm{~L}_{21} . \mathrm{L}_{22}$ est évidemment $\mathrm{L}_{2}$.

$\mathrm{L}_{3}$ s'écrit: $S_{2}=H^{2} \Lambda_{3}$; on définit $\mathrm{L}_{31}: S_{2}=H \Lambda_{31} ;$ si $\mathrm{L}_{2}$ est vérifiée, $\mathrm{L}_{31}$ équivaut à $\mathrm{L}_{(3) 2} ; \mathrm{L}_{32}$ est évidemment $\mathrm{L}_{3}$.

$\mathrm{L}_{4}$ s'écrit: $S_{3}=H^{2} \Lambda_{4}$; la divisibilité par $H$ est une conséquence de $\mathrm{L}_{1}, \mathrm{~L}_{2}, \mathrm{~L}_{3}$.

5) Type $(2,2,1)$. La première condition $\mathrm{L}$ est $\mathrm{L}_{2}: S_{1}=H \Lambda_{2}$; on définit:

$$
\mathrm{L}_{11}: S_{0} \equiv \Lambda_{1}=H \Lambda_{11}
$$

Si $\mathrm{L}_{2}$ est vérifiée, on pose: $S_{2}=\Lambda_{30}$; on a:

$$
a \Lambda_{30}=H^{2} K \sigma_{\mu_{2}}\left[h \Lambda_{2}^{\prime}-h \Lambda_{1}^{\prime} H^{\prime} K^{\prime}+h \mathcal{A}^{\prime} H^{2} K^{\prime} H^{\prime} K^{\prime}-{H^{\prime}}^{2} K^{\prime} H^{\prime} K^{\prime} H K^{\prime}\right]
$$

La condition $\mathrm{L}_{(4) 1}$ s'écrit:

$$
\begin{aligned}
\mathcal{A} \sigma_{\mu_{(3)}}\left[h \Lambda_{30}^{\prime}-h \Lambda_{2}^{\prime}{H^{\prime}}^{2} K^{\prime}+h \Lambda_{1}^{\prime} H^{\prime} K^{\prime}{H^{\prime}}^{2} K^{\prime}-h \mathcal{A}^{\prime}{H^{\prime}}^{2} K^{\prime} H^{\prime} K^{\prime}{H^{\prime}}^{2} K^{\prime}\right. \\
\left.+{H^{\prime}}^{2} K^{\prime} H^{\prime} K^{\prime} H^{\prime} K^{\prime}{H^{\prime}}^{2} K^{\prime}\right]=H \Lambda_{(4) 1}
\end{aligned}
$$

$\mu_{(3)}=\mu_{3}+s$.

Si $L_{11}$ est vérifiée, $L_{(4) 1}$ est vérifiée.

$\mathrm{L}_{3}$ s'écrit $S_{2}=H \Lambda_{3}$. Si $\mathrm{L}_{3}$ est vérifiée, alors $\mathrm{L}_{(4) 1}$ est vérifiée.

Si $L_{11}$ est vérifiée, alors $L_{3}$ est vérifiée.

$\mathrm{L}_{4}$ s'écrit: $S_{3}=H \Lambda_{4}$.

$\mathrm{L}_{5}$ s'écrit: $S_{4}=H \Lambda_{5}$.

$\mathrm{L}_{6}$ s'écrit: $S_{5}=H \Lambda_{6}$. Si $\mathrm{L}_{11}$ n'est pas vérifiée, $\mathrm{L}_{1}, \mathrm{~L}_{2}, \mathrm{~L}_{3}, \mathrm{~L}_{4}, \mathrm{~L}_{5}$ impliquent $\mathrm{L}_{6}$.

6) Type $(2,1,1,1) . \quad \mathrm{L}_{1}$ s'écrit: $S_{0}=H \Lambda_{1} ; \mathrm{L}_{2}: S_{1}=H \Lambda_{2} ; \mathrm{L}_{3}: S_{2}=H \Lambda_{3}$; $\mathrm{L}_{4}: S_{3}=H \Lambda_{4}$.

Lemme 1. Les conditions précédentes ne dépendent pas du choix des opérateurs $H^{\prime}, K^{\prime}, \mathcal{A}^{\prime}, \Lambda_{1}^{\prime}, \ldots$

0.3. Pour définir les conditions $(\mathrm{LG})_{d}, d>1$, qui permettront d'obtenir les conditions nécessaires et suffisantes pour que le problème de Cauchy soit bien posé dans les classes de Gevrey $\gamma^{d}$, nous étudierons les diagrammes $\mathcal{N}$ de chaque type.

DÉfinition 6. Soit un sous-ensemble $E$ de $\mathcal{N}$, l'enveloppe convexe de la réunion de 0 et du sous-ensemble $E$ par le haut a pour frontière des segments 
de droite non verticaux. On appellera diagramme de Newton $N$ du sous-ensemble $E$ la réunion de 0 et des points du sous-ensemble qui sont sur ces segments de droite.

1) Type (5). $m_{1}=5, p=5 ; r=4 . \mathcal{N}$ est tel que: $0<n_{1} \leq 5 ; 0<n_{2} \leq 4$; $5 n_{2} \leq 4 n_{1}$.

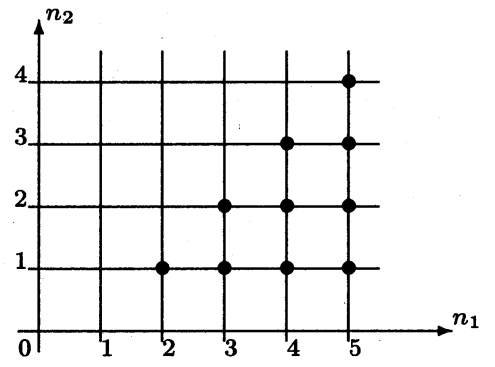

1) Le sous-ensemble $E$ est $\mathcal{N}$ lui même. $N$ est formé de 0 et du point $(5,4)$. La pente du 1 er segment est $\frac{4}{5}$. On définit $(L G)_{5 / 4}: L_{11}$ n'est pas vérifiée. La classe de Gevrey correspondant est $\gamma^{d}: d=\frac{p}{p-1}=\frac{5}{4} ; d$ est l'inverse de la pente du premier segment en partant de la gauche.

2) On supprime le point $(5,4) . N$ est formé de 0 et des points: $(4,3)$ et $(4,4)$. La pente du 1er segment est $\frac{3}{4}$. On définit: $(L G)_{4 / 3}: L_{11}$ est vérifiée, $L_{12}$ n'est pas vérifiée; comme précédemment, on obtient: $d=\frac{4}{3}$.

3) On supprime le point $(4,3) . N$ est formé des points $0,(3,2),(5,3)$. La pente du 1er segment est $\frac{2}{3}$. On définit $(L G)_{3 / 2} ; L_{12}$ est vérifiée, $L_{13}$ n'est pas vérifiée: $d=\frac{3}{2}$.

4) On supprime le point $(3,2)$. $N$ est formé des points 0 et $(5,3)$. La pente du 1er segment est $\frac{3}{5}$. $(\mathrm{LG})_{5 / 3}: \mathrm{L}_{13}$ est vérifiée; $\mathrm{L}_{(2) 3}$ n'est pas vérifiée: $d=\frac{5}{3}$.

5) On supprime le point $(5,3)$. $N$ est formé des 4 points: $0,(2,1),(4,2),(5,2)$. La pente du 1er segment est $\frac{1}{2}$. On définit $(L G)_{2}: L_{13}$ est vérifiée, $L_{(2) 3}$ est vérifiée; de plus ou bien ( $\mathrm{L}_{1}$ n'est pas vérifiée) ou bien ( $\mathrm{L}_{23}$ n'est pas vérifiée), $d=2$.

6) On supprime les points $(2,1)$ et $(4,2)$. $N$ est formé des points 0 et $(5,2)$. La pente du 1er segment est $\frac{2}{5}$. On définit $(L G)_{5 / 2}: L_{1}$ est vérifiée, $L_{23}$ est vérifiée, $\mathrm{L}_{(3) 4}$ n'est pas vérifiée: $d=\frac{5}{2}$.

7) On supprime le point $(5,2)$. $N$ est formé des points $0,(3,1),(4,1),(5,1)$. La pente du 1er segment est $\frac{1}{3}$. On définit $(\mathrm{LG})_{3}: \mathrm{L}_{1}, \mathrm{~L}_{23}, \mathrm{~L}_{(3) 4}$ sont vérifiées, $\mathrm{L}_{2}$ n'est pas vérifiée: $d=3$.

8) On supprime le point $(3,1) . N$ est formé de $0,(4,1),(5,1)$. La pente du $1 \mathrm{er}$ segment est $\frac{1}{4}$. On définit: $(L G)_{4}: L_{1}, L_{2}, L_{33}$ sont vérifiées, $L_{3}$ n'est pas vérifiée: $d=4$. 
9) On supprime le point $(4,1) . N$ est formé de 0 et de $(5,1)$. La pente du 1er segment est $\frac{1}{5}$. On définit $(\mathrm{LG})_{5}: \mathrm{L}_{1}, \mathrm{~L}_{2}, \mathrm{~L}_{3}$ sont vérifiées, $\mathrm{L}_{4}$ n'est pas vérifiée $d=5$.

2) Type $(4,1) . \quad m_{1}=5 ; p=4 ; r=3 . \mathcal{N}$ est tel que: $0<n_{1} \leq 5$, $0<n_{2} \leq 3,4 n_{2} \leq 3 n_{1} ; c=9$.

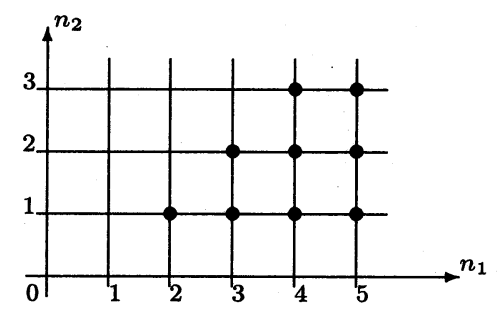

1) Le sous-ensemble de $\mathcal{N}$ est $\mathcal{N}$ lui-même. $N$ est formé de $0,(4,3)$ et de $(5,3)$. La pente du 1 er segment est $\frac{3}{4}$. On définit $(L G)_{4 / 3}: L_{11}$ n'est pas vérifiée: $d=\frac{p}{p-1}=\frac{4}{3}$.

2) On supprime le point $(4,3) . N$ est formé de $0,(3,2)$ et $(5,3)$. La pente du 1er segment est $\frac{2}{3}$. On définit $(L G)_{3 / 2}: L_{11}$ est vérifiée $L_{12}$ n'est pas vérifiée: $d=\frac{3}{2}$.

3) On supprime le point: $(3,2)$. $N$ est formé des points $0,(5,3)$. La pente du 1er segment est $\frac{3}{5}$. On définit $(L G)_{5 / 3}: L_{12}$ est vérifiée, $L_{(2) 2}$ n'est pas vérifiée: $d=\frac{5}{3}$.

4) On supprime le point $(5,3) . N$ est formé des points: $0,(2,1),(4,2),(5,2)$. La pente du 1er segment est $\frac{1}{2}$. On définit $(\mathrm{LG})_{2}:\left(\mathrm{L}_{12}\right.$ et $\mathrm{L}_{(2) 2}$ sont vérifiées, $\mathrm{L}_{1}$ n'est pas vérifiée) ou ( $\mathrm{L}_{1}, \mathrm{~L}_{21}$ sont vérifiées, $\mathrm{L}_{22}$ n'est pas vérifiée). $d=2$.

5) On supprime les points $(2,1)$ et $(4,2)$. $N$ est formé des points 0 et $(5,2)$. La pente du ler segment est $\frac{2}{5}$. On définit $(L G)_{5 / 2}: L_{1}, L_{2}$ sont vérifiées, $L_{(3) 3}$ n'est pas vérifiée: $d=\frac{5}{2}$.

6) On supprime le point $(5,2)$. $N$ est formé des points $0,(3,1),(4,1)$ et $(5,1)$. La pente du 1er segment est $\frac{1}{3}$. On définit $(\mathrm{LG})_{3}: \mathrm{L}_{11}, \mathrm{~L}_{22}, \mathrm{~L}_{(3) 3}$ sont vérifiées, $\mathrm{L}_{2}$ n'est pas vérifiée: $d=3$.

7) On supprime le point $(3,1) . \quad N$ est formé des points $0,(4,1)$ et $(5,1)$. La pente du ler segment est $\frac{1}{4}$. On définit $(L G)_{4}: L_{1}, L_{2}, L_{32}$ sont vérifiées, $L_{3}$ n'est pas vérifiée. $d=4$.

8) On supprime le point $(4,1) . N$ est formé des points 0 et $(5,1)$. La pente du 1er segment est $\frac{1}{5}$. On définit $(L G)_{5}: L_{1}, L_{2}, L_{3}$ sont vérifiées, $L_{4}$ n'est pas vérifiée. $d=5$.

3) Type (3,2). $\quad m_{1}=5 ; p=3 ; r=3 . \quad \mathcal{N}$ est tel que: $0<n_{1} \leq 5$, $0<n_{2} \leq 3 ; 3 n_{2} \leq 2 n_{1} ; c=8$. 


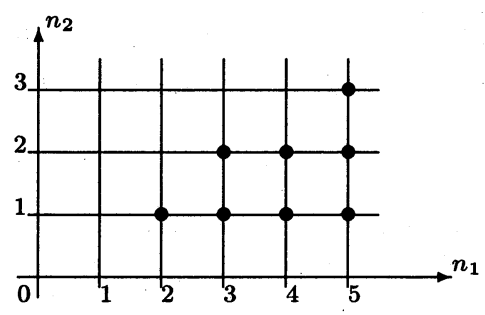

1) Le sous-ensemble de $\mathcal{N}$ est $\mathcal{N}$ lui-même. $N$ est formé de 0 et $(3,2)$ et de $(5,3)$. La pente du 1er segment est $\frac{2}{3}$. On définit: $(L G)_{3 / 2}: L_{1}$ n'est pas vérifiée: $d=\frac{p}{p-1}=\frac{3}{2}$.

2) On supprime le point $(3,2)$. $N$ est formé de 0 et de $(5,3)$. La pente du 1er segment est $\frac{3}{5}$. On définit $(\mathrm{LG})_{5 / 3}: \mathrm{L}_{1}$ est vérifiée, $\mathrm{L}_{21}$ n'est pas vérifiée: $d=\frac{5}{3}$.

3) On supprime le point $(5,3) . \quad N$ est formé de $0,(2,1),(4,2)$ et $(5,2)$. La pente du ler segment est $\frac{1}{2}$. On définit $(L G)_{2}:\left(L_{1}, L_{21}\right.$ sont vérifiées et $L_{2}$ ne l'est pas) ou ( $\mathrm{L}_{12}$ est vérifiée, $\mathrm{L}_{3}$ ne l'est pas). $d=2$.

4) On supprime les points $(2,1)$ et $(4,2) . N$ est formé de 0 et $(5,2)$. La pente du 1er segment est $\frac{2}{5}$. On définit $(\mathrm{LG})_{5 / 2}:\left(\mathrm{L}_{1}, \mathrm{~L}_{2}\right)$ sont vérifiées, $\mathrm{L}_{12}$ et $\mathrm{L}_{(4) 2}$ ne sont pas vérifiées; $d=\frac{5}{2}$.

5) On supprime le point $(5,2)$. $\quad N$ est formé de $0,(3,1),(4,1)$ et $(5,1)$. La pente du 1er segment est $\frac{1}{3}$. On définit: $(L G)_{3}:\left(L_{12}\right.$ n'est pas vérifiée; $L_{1}$, $\mathrm{L}_{2}, \mathrm{~L}_{(4) 2}$ sont vérifiées, $\mathrm{L}_{3}$ n'est pas vérifiée) ou $\left(\mathrm{L}_{12}, \mathrm{~L}_{3}\right.$ sont vérifiées, $\mathrm{L}_{4}$ ne l'est pas). $d=3$.

6) On supprime le point $(3,1) . N$ est formé de $0,(4,1)$ et $(5,1)$. La pente du ler segment est $\frac{1}{4}$. On définit $(L G)_{4}:\left(L_{12}\right.$ n'est pas vérifiée; $L_{1}, L_{2}, L_{3}$ sont vérifiées, $\mathrm{L}_{4}$ ne l'est pas) ou ( $\mathrm{L}_{12}, \mathrm{~L}_{3}, \mathrm{~L}_{4}$ sont vérifiées, $\mathrm{L}_{6}$ ne l'est pas). $d=4$.

7) On supprime le point $(4,1) . N$ est formé de 0 et de $(5,1)$. La pente du ler segment est $\frac{1}{5}$. On définit $(L G)_{5}:\left(L_{12}\right.$ n'est pas vérifiées, $L_{1}, L_{2}, L_{3}, L_{4}$ sont vérifiées, $\mathrm{L}_{5}$ ne l'est pas) ou $\left(\mathrm{L}_{12}, \mathrm{~L}_{3}, \mathrm{~L}_{4}, \mathrm{~L}_{6}\right.$ sont vérifiées, $\mathrm{L}_{8}$ n'est pas vérifiée). $d=5$.

4) Type $(3,1,1) . \quad m_{1}=5 ; p=3 ; r=2 ; \mathcal{N}$ est tel que: $0<n_{1} \leq 5$; $0<n_{2} \leq 2 ; 3 n_{2} \leq 2 n_{1} ; c=7$.

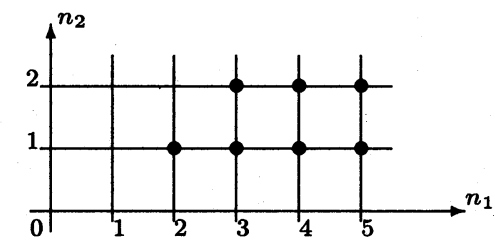


1) Le sous-ensemble de $\mathcal{N}$ est $\mathcal{N}$ lui-même. $N$ est formé de $0,(3,2),(4,2)$ et $(5,2)$. La pente du ler segment est $\frac{2}{3}$. On définit $(L G)_{3 / 2}: L_{11}$ n'est pas vérifiée. $d=\frac{p}{p-1}=\frac{3}{2}$.

2) On supprime le point: $(3,2) . \quad N$ est formé de $0,(2,1),(4,2)$ et $(5,2)$. La pente du ler segment est $\frac{1}{2}$. On définit: $(L G)_{2}:\left(L_{11}\right.$ est vérifiée, $L_{1}$ n'est pas vérifiée) ou ( $\mathrm{L}_{1}$ est vérifiée, $\mathrm{L}_{21}$ n'est pas vérifiée): $d=2$.

3) On supprime les points: $(2,1)$ et $(4,2) . N$ est formé de 0 et $(5,2)$. La pente du 1er segment est $\frac{2}{5}$. On définit $(\mathrm{LG})_{5 / 2}: \mathrm{L}_{1}, \mathrm{~L}_{21}$ sont vérifiées, $\mathrm{L}_{(3) 2}$ n'est pas vérifiée: $d=5 / 2$.

4) On supprime le point $(5,2)$. $\quad N$ est formé de $0,(3,1),(4,1)$ et $(5,1)$. La pente du 1 er segment est $\frac{1}{3}$. On définit $(L G)_{3}: L_{1}, L_{21}, L_{(3) 2}$ sont vérifiées, $\mathrm{L}_{2}$ ne l'est pas: $d=3$.

5) On supprime le point $(3,1) . N$ est formé de $0,(4,1)$ et $(5,1)$. La pente du 1er segment est $\frac{1}{4}$. On définit $(L G)_{4}: L_{1}, L_{2}, L_{31}$ sont vérifiées, $L_{3} n$ 'est pas vérifiée: $d=4$.

6) On supprime le point $(4,1)$. $N$ est formé de 0 et $(5,1)$. La pente du 1er segment est $\frac{1}{5}$. On définit $(\mathrm{LG})_{5} ; \mathrm{L}_{1}, \mathrm{~L}_{2}, \mathrm{~L}_{3}$ sont vérifiées, $\mathrm{L}_{4}$ ne l'est pas: $d=5$.

5) Type (2,2,1). $\quad m_{1}=5 ; p=2 ; r=2 ; \mathcal{N}$ est tel que: $0<n_{1} \leq 5$; $0<n_{2} \leq 2 ; 2 n_{2} \leq n_{1} ; c=6$.

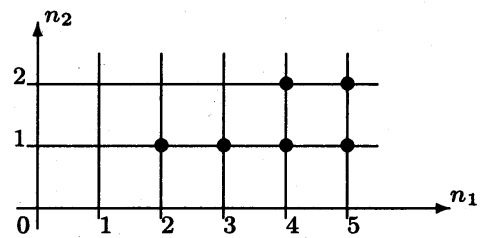

1) Le sous-ensemble de $\mathcal{N}$ est $\mathcal{N}$ lui-même. $N$ est formé de $0,(2,1),(4,2)$ et $(5,2)$. La pente du ler segment est $\frac{1}{2}$. On définit $(L G)_{2}: L_{2}$ n'est pas vérifiée: $d=\frac{p}{p-1}=2$.

2) On supprime les points $(2,1)$ et $(4,2) . N$ est formé de 0 et $(5,2)$. La pente du 1er segment est $\frac{2}{5}$. On définit $(L G)_{5 / 2}: L_{2}$ est vérifiée, $L_{(4) 1}$ n'est pas vérifiée; $d=5 / 2$.

3) On supprime le point: $(5,2)$. $N$ est formé de $0,(3,1),(4,1)$ et $(5,1)$. La pente du 1er segment est $\frac{1}{3}$. On définit $(\mathrm{LG})_{3}:\left(\mathrm{L}_{2}, \mathrm{~L}_{(4) 1}\right.$ sont vérifiées, $\mathrm{L}_{3} \mathrm{n}$ 'est pas vérifiée) ou ( $\mathrm{L}_{11}$ est vérifiée, $\mathrm{L}_{4} \mathrm{n}$ 'est pas vérifiée): $d=3$.

4) On supprime le point: $(3,1)$. $N$ est formé de $0,(4,1)$ et $(5,1)$. La pente du 1er segment est $\frac{1}{4}$. On définit $(L G)_{4}:\left(L_{2}, L_{3}\right.$ sont vérifiées, $L_{11}, L_{4}$ ne sont pas vérifiées) ou ( $\mathrm{L}_{11}, \mathrm{~L}_{4}$ sont vérifiées, $\mathrm{L}_{5}$ n'est pas vérifiée): $d=4$. 
5) On supprime le point $(4,1)$. $N$ est formé de 0 et $(5,1)$. La pente du 1er segment est $\frac{1}{5}$. On définit $(\mathrm{LG})_{5}:\left(\mathrm{L}_{2}, \mathrm{~L}_{3}, \mathrm{~L}_{4}\right.$ sont vérifiées, $\mathrm{L}_{11}, \mathrm{~L}_{5}$ ne sont pas vérifiées) ou ( $\mathrm{L}_{11}, \mathrm{~L}_{4}, \mathrm{~L}_{5}$ sont vérifiées, $\mathrm{L}_{6}$ n'est pas vérifiée): $d=5$.

6) Type $(2,1,1,1) . \quad m_{1}=5 ; p=2, r=1 ; \mathcal{N}$ est tel que: $0<n_{1} \leq 5$; $0<n_{2} \leq 1 ; 2 n_{2} \leq n_{1} ; c=4$.

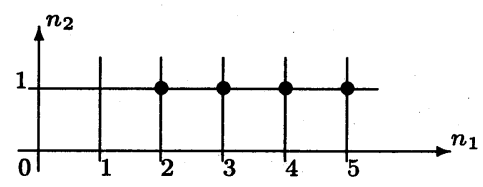

1) Le sous-ensemble $\mathcal{N}$ est $\mathcal{N}$ lui-même. $N$ est formé de $0,(2,1),(3,1),(4,1)$ et $(5,1)$. La pente du 1er segment est $\frac{1}{2}$. On définit $(L G)_{2}: L_{1}$ n'est pas vérifiée: $d=\frac{p}{p-1}=2$.

2) On supprime le point $(2,1) . \quad N$ est formé de $0,(3,1),(4,1)$ et $(5,1)$. La pente du ler segment est $\frac{1}{3}$. On définit $(L G)_{3}: L_{1}$ est vérifiée, $L_{2} n$ 'est pas vérifiée: $d=3$

3) On supprime le point $(3,1), N$ est formé de $0,(4,1)$ et $(5,1)$. La pente du 1er segment est $\frac{1}{4}$. On définit $(\mathrm{LG})_{4}: \mathrm{L}_{1}, \mathrm{~L}_{2}$ sont vérifiées, $\mathrm{L}_{3}$ ne l'est pas: $d=4$.

4) On supprime le point $(4,1), N$ est formé de 0 et de $(5,1)$. La pente du 1er segment est $\frac{1}{5}$. On définit $(\mathrm{LG})_{5}: \mathrm{L}_{1}, \mathrm{~L}_{2}, \mathrm{~L}_{3}$ sont vérifiées; $\mathrm{L}_{4}$ ne l'est pas: $d=5$.

Remarque. Les conditions $(\mathrm{LG})_{d}$ sont évidemment invariantes.

Proposition 3. Les conditions (LG) de dépendent pas du choix des opérateurs $H^{\prime}, K^{\prime}, \mathcal{A}, \Lambda_{1}, \ldots$. $h$.

Conséquence. On peut les écrire explicitement à l'aide des coefficients de

Remarque. Lorsque la 1ère condition $\mathrm{L}$ n'est pas vérifiée, on démontre que dans tous les types, la classe de Gevrey convenable est $\gamma^{p / p-1}$; ce résultat sera démontré ailleurs. Il est plus difficile de prouver que si on impose aucune condition la classe de Gevrey est encore $\gamma^{p / p-1}$; c'est toutefois prouvé dans cet article pour $m_{1} \leq 5$.

0.4. La réduction de l'opérateur $h$ utilise essentiellement la

Proposition 4. $\Delta$ est un opérateur matriciel pseudodifférentiel analytique d'ordre 0 et inversible; $\tilde{h}=\Delta^{-1} h \Delta . \quad$ Si $h$ vérifie les conditions $\mathrm{L}$ (resp. $\left.(\mathrm{LG})_{d}\right)$ d'un type, $\tilde{h}$ les vérifie aussi et réciproquement.

On utilise des réductions maintenant classiques [3], [7], [11], [15], [22], [25], en particulier, celle d'Arnold Petkov, [17]. On désigne par $\Sigma_{1}$ l'ensemble analy- 
tique de codimension $\geq 1$ de changement de rang [22], c'est le complémentaire de l'ensemble des $\left(x, \xi^{\prime}\right)$ pour lequel le rang généralisé est constant; on désigne par $\Sigma_{2}$ une réunion finie d'ensembles analytiques, de codimensions $\geq 1$ obtenus comme il suit: $r\left(x, \xi^{\prime}\right)$ sera un symbole analytique dans un voisinage conique, construit à partir des coefficients de $\tilde{h}$. On sera amené à distinguer les cas $r\left(x, \xi^{\prime}\right) \neq 0, \forall x, \xi^{\prime} \neq 0$; $r\left(x, \xi^{\prime}\right) \equiv 0$; le cas $r\left(x, \xi^{\prime}\right) \not \equiv 0$ et $r\left(x, \xi^{\prime}\right)$ peut s'annuler définit un sous ensemble analytique de codimension $\geq 1 \Sigma_{2}: r\left(x, \xi^{\prime}\right)=0$. On désigne par $\Sigma$ la réunion de $\Sigma_{1}$ et $\Sigma_{2}$ qui est donc de codimension $\geq 1$; dans chaque voisinage conique considéré le complémentaire de $\Sigma$ sera un ouvert conique analytique partout dense.

On peut encore dire comme [11] que nos calculs portent sur des symboles formels méromorphes.

On obtient donc la forme réduit $\tilde{h}$ de l'opérateur au voisinage conique de chaque point, en dehors de $\Sigma$.

$\tilde{h}$ est un opérateur matriciel $m_{1} \times m_{1}$ pseudodifférentiel analytique d'ordre 1 ;

$$
\tilde{h}=\tilde{a}+\tilde{b}=I D_{0}+\mathrm{a}\left|D^{\prime}\right|+\tilde{b}
$$

a est la matrice formé de blocs de Jordan de dimension $p, q_{1}, \ldots, q_{\ell} ; r$ est le rang générique de $\tilde{a}$ aux points caractéristiques; nous l'expliciterons dans chaque type; $b$ est pseudodifférentiel d'ordre 0 ,

$$
\tilde{b}=\tilde{b}_{0}+\tilde{b}_{1}+\cdots+\tilde{b}_{k}+\cdots,
$$

$\tilde{b}_{k}$ est d'ordre $-k$.

Si $\tilde{h}$ satisfait les conditions L ou (LG) $)_{d}$ du cas considéré $\tilde{h}$ les satisfait aussi; de même si chaque $\tilde{h}$ vérifie ces conditions, $h$ les vérifie.

Nous expliciterons les conditions $\mathrm{L},(\mathrm{LG})_{d}$ de chaque type et dans chaque cas à l'aide des coefficients de $\tilde{h}$ sous la forme réduite.

On a aussi la

Proposition 5. Si $h$ vérifie les conditions $\mathrm{L}$ ou $(\mathrm{LG})_{d}$, le transposé de $h$ les vérifie aussi.

0.5. HK se décompose sous la forme:

$$
H K=\left(\xi_{0}-\lambda_{1}\right) \cdots\left(\xi_{0}-\lambda_{s}\right)\left(\xi_{0}-\lambda_{s+1}\right) \cdots\left(\xi_{0}-\lambda_{s+\chi}\right)
$$

On cherche une paramétrixe $F$ qui devra vérifier dans les cas $(\mathrm{LG})_{d}$ ou L les relations:

$$
\begin{cases}h \circ F=0 & \text { modulo un opérateur d'ordre }-\infty \\ \left.F\right|_{x_{0}=\underline{x}_{0}}=I & \text { modulo un opérateur d'ordre }-\infty\end{cases}
$$


Comme usuellement, [1], [7], [18] on se ramène à la construction de paramétrixes $F_{j}$ relatives à chaque racine caractéristique $\lambda_{j}$, l'identité $I$ se décompose à l'aide d'un déterminant de Vandermonde des $\lambda_{j}$ et donne les conditions initiales $I_{j}$ pour chaque paramétrixe $F_{j}$.

$F_{j}$ sera cherchée de la forme:

$$
F_{j} u(x)=\frac{1}{(2 \pi)^{n}} \int e^{\varphi_{j}\left(x, \eta^{\prime}\right)} f_{j}\left(x, \eta^{\prime}\right) \hat{u}\left(\underline{x}_{0}, \eta^{\prime}\right) d \eta^{\prime}
$$

où: $\varphi_{j}$ vérifie: $D_{0} \varphi_{j}-\lambda_{j}\left(x, D^{\prime} \varphi_{j}\right)=0, \varphi_{j}\left(\underline{x}_{0}, x^{\prime}, \eta^{\prime}\right)=x^{\prime} \cdot \eta^{\prime} ; \hat{u}\left(\underline{x}_{0}, \eta^{\prime}\right)$ est la transformée de Fourier de $u\left(\underline{x}_{0}, y^{\prime}\right)$ en $y^{\prime}$; après les réductions, on est ramené à chercher des paramétrixes microlocales de la forme:

$$
\tilde{F}_{j} v(\tilde{x})=\frac{1}{(2 \pi)^{n}} \int e^{i x^{\prime} \xi^{\prime}} \tilde{f}_{j}\left(\tilde{x}, \eta^{\prime}\right) \hat{v}\left(\underline{x}_{0}, \eta^{\prime}\right) d \eta^{\prime}
$$

où: $\tilde{f}_{j}\left(\underline{x}_{0}, \tilde{x}, \eta^{\prime}\right)$ est une somme asymptotique formelle "méromorphe", $\tilde{f}_{j}$ est une somme finie de termes de la forme i) ou ii) (en omettant de réécrire ${ }^{\sim}$ et $j$ ):

$$
\text { i) } f_{1}=e^{\psi\left(x, \eta^{\prime}\right)} Y\left(x, \eta^{\prime}\right)
$$

$\psi\left(x, y^{\prime}\right)$ est une somme finie de symboles analytiques d'ordre fractionnaire:

$$
\psi=\psi_{1}\left(x, \eta^{\prime}\right)+\psi_{2}+\cdots
$$

$\psi_{1}$ est d'ordre $1 / d$ en $\eta^{\prime}, d>1 ; \psi_{2}, \ldots$ sont d'ordre strictement inférieurs; $\psi\left(\underline{x}_{0}, y^{\prime}, \eta^{\prime}\right)=0$

$$
Y=Y_{0}+\cdots+Y_{k}+\cdots
$$

les symboles analytiques matriciels $m_{1} \times m, Y_{k}$ sont d'ordres fractionnaires décroissants qui seront précisés dans chaque cas.

$$
\text { ii) } f_{2}=Z\left(x, \eta^{\prime}\right)
$$

$Z=Z_{0}+\cdots+Z_{k}+\cdots ;$ les $Z_{k}$ sont des symboles analytiques matriciels $m_{1} \times m_{1}$ d'ordres fractionnaires décroissants. On aura de plus des conditions initiales de la forme: $f\left(\underline{x}_{0}, x^{\prime}, \eta^{\prime}\right)=I_{m_{1}}, I_{m_{1}}$ matrice $m_{1} \times m_{1}$.

On utilisera la formule de composition des opérateurs: si $b$ et $\mathcal{Y}$ sont des sommes asymptotiques formelles, le composé $b \circ \mathcal{Y}$ est la somme: $\Sigma r_{j}$, où:

$$
r_{j}\left(x, \eta^{\prime}\right)=\sum_{\left|\alpha^{\prime}\right|+k+\ell=j}\left(\alpha^{\prime} !\right)^{-1} \partial_{\alpha^{\prime}} b_{k}\left(x, \eta^{\prime}\right) D^{\alpha^{\prime}} \mathcal{Y}_{\ell}\left(x, \eta^{\prime}\right)
$$


on a posé comme usuellement:

$$
\begin{gathered}
D^{\alpha^{\prime}}=D_{1}^{\alpha_{1}} \ldots D_{n}^{\alpha_{n}}, \quad D_{j}=D_{x_{j}}, \quad D_{x^{\prime}}=\left(D_{1}, D_{j}, \ldots, D_{n}\right) \\
\partial^{j}=\frac{\partial}{\partial \xi_{j}}, \quad \partial_{\xi^{\prime}}=\left(\partial^{1}, \ldots, \partial^{n}\right) \\
\partial_{\alpha^{\prime}}=\left(\partial^{1}\right)^{\alpha_{1}} \ldots\left(\partial^{n}\right)^{\alpha_{n}}, \quad D_{0}=\frac{\partial}{\partial x_{0}}
\end{gathered}
$$

Nous donnerons dans les paragraphes suivants le calcul de coefficients $Y_{k}$ et $Z_{k}$ et par suite les expressions formelles des paramétrixes. On posera aussi pour simplifier les notations dans ces paragraphes:

$$
\left|\eta^{\prime}\right|=|\eta| ; \quad \frac{\xi^{\prime}}{|\xi|} \cdot D_{x^{\prime}}=D_{*} ; \quad \sum_{1 \leq j \leq n} \frac{\partial b_{k}}{\partial \xi_{j}^{\prime}} D_{x j}=\left(D b_{k}\right)_{*} .
$$

On notera simplement $\tilde{h}=h$. On notera: $W\left(\psi_{1}, \psi_{2}, \ldots ; \sigma\right)$, des termes polynômiaux en dérivées de $\psi_{1}, \psi_{2}, \ldots$, d'ordre $\sigma$, que l'on ne détaillera pas.

0.6. Dans le dernier paragraphe, on énoncera les applications des calculs précédents. On montrera que ces calculs peuvent s'interpréter en terme de développements asymptotiques d'où l'on déduit que les conditions $\mathrm{L}$ sont nécessaires pour que le problème de Cauchy soit bien posé en $\mathcal{C}^{\infty}$. Ils permettent aussi d'obtenir les conditions suffisantes en $\mathcal{C}^{\infty}$ et en Gevrey. La nécessité des conditions $(\mathrm{LG})_{d}$ pour que le problème de Cauchy soit bien posé en Gevrey sera faite dans une autre publication.

\section{§1. Étude du type (5)}

$$
h=\left(\begin{array}{ccccc}
D_{0} & \left|D^{\prime}\right| & 0 & 0 & 0 \\
0 & D_{0} & \left|D^{\prime}\right| & 0 & 0 \\
0 & 0 & D_{0} & \left|D^{\prime}\right| & 0 \\
0 & 0 & 0 & D_{0} & \left|D^{\prime}\right| \\
b_{1}^{5} & b_{2}^{5} & b_{3}^{5} & b_{4}^{5} & D_{0}
\end{array}\right)
$$

Les conditions $(\mathrm{LG})_{d}$ s'écrivent:

$$
\begin{aligned}
& (\mathrm{LG})_{5 / 4}: b_{01}^{5} \neq 0 \\
& (\mathrm{LG})_{4 / 3}: b_{01}^{5}=0, \quad b_{02}^{5} \neq 0 \\
& (\mathrm{LG})_{3 / 2}: b_{01}^{5}=b_{02}^{5}=0, \quad b_{03}^{5} \neq 0 \\
& (\mathrm{LG})_{5 / 3}: b_{01}^{5}=b_{02}^{5}=b_{03}^{5}=0, \quad b_{11}^{5} \neq 0
\end{aligned}
$$




$$
\begin{aligned}
&(\mathrm{LG})_{2}: b_{01}^{5}=b_{02}^{5}=b_{03}^{5}=b_{11}^{5}=0, \quad b_{04}^{5} \neq 0 \text { ou } b_{12}^{5} \neq 0 \\
&(\mathrm{LG})_{5 / 2}: b_{01}^{5}=b_{02}^{5}=b_{03}^{5}=b_{04}^{5}=b_{11}^{5}=b_{12}^{5}=0, \quad b_{21}^{5} \neq 0 \\
&(\mathrm{LG})_{3}: b_{01}^{5}=b_{02}^{5}=b_{03}^{5}=b_{04}^{5}=b_{11}^{5}=b_{12}^{5}=b_{21}^{5}=0, \quad b_{13}^{5} \neq 0 \\
&(\mathrm{LG})_{4}: b_{01}^{5}=b_{02}^{5}=b_{03}^{5}=b_{04}^{5}=b_{11}^{5}=b_{12}^{5}=b_{13}^{5}=b_{21}^{5}=0, \quad b_{22}^{5} \neq 0 \\
&(\mathrm{LG})_{5}: b_{01}^{5}=b_{02}^{5}=b_{03}^{5}=b_{04}^{5}=b_{11}^{5}=b_{12}^{5}=b_{13}^{5}=b_{21}^{5}=b_{22}^{5}=0, \quad b_{31}^{5} \neq 0
\end{aligned}
$$

Les conditions L s'écrivent:

$$
b_{01}^{5}=b_{02}^{5}=b_{03}^{5}=b_{04}^{5}=b_{11}^{5}=b_{12}^{5}=b_{13}^{5}=b_{21}^{5}=b_{22}^{5}=b_{31}^{5}=0 .
$$

1.1. Cas $(\mathbf{L G})_{5 / 4}$. On cherche $f$ sous la forme:

$$
\begin{aligned}
f & =\sum_{1 \leq t \leq 5} f^{t} \\
f^{t} & =e^{\psi_{1}^{t}+\psi_{2}^{t}+\psi_{3}^{t}+\psi_{4}^{t}}\left(Y_{0}^{t}+\cdots+Y_{k}^{t}+\cdots\right) .
\end{aligned}
$$

Les 5 phases complexes distinctes $\psi_{1}^{t}, 1 \leq t \leq 5$ vérifient:

$$
\left(D_{0} \psi_{1}^{t}\right)^{5}=-b_{01}^{5}|\eta|^{4} \neq 0 ;\left.\quad \psi_{1}^{t}\right|_{x_{0}=\underline{x}_{0}}=0 ;
$$

chaque $\psi_{1}^{t}$ est d'ordre: $4 / 5$.

Les 5 phases $\psi_{2}^{t}$ vérifient:

$$
D_{0} \psi_{2}^{t}=\frac{4}{5|\eta|} D_{0} \psi_{1}^{t} D_{*} \psi_{1}^{t}-\frac{b_{02}^{5}\left(D_{0} \psi_{1}\right)^{2}}{5 b_{01}^{5}|\eta|}-\frac{\left(D b_{01}^{5}\right)_{*}\left(\psi_{1}\right) D_{0} \psi_{1}}{5 b_{01}^{5}} ;\left.\quad \psi_{2}^{t}\right|_{x_{0}=\underline{x}_{0}}=0
$$

chaque $\psi_{2}^{t}$ est d'ordre: $3 / 5$.

On définit d'une façon analogue les $\psi_{3}^{t}$ et les $\psi_{4}^{t}$ d'ordres $2 / 5$ et $1 / 5$; leur choix sera expliqué par la suite.

$Y_{k}^{t}$ est d'ordre: $\frac{4-k}{5}$.

Pour que $h \circ f=0$, modulo un opérateur d'ordre $-\infty$, il suffit que pour chaque $t$ le système différentiel suivant en $Y_{0}^{t}, \ldots, Y_{k}^{t}, \ldots$, obtenu en annulant les parties homogènes des symboles, soit vérifié. [Pour simplifier la typographie, nous omettons l'indice $t$ dans l'écriture et l'étude du système] [les $c_{\alpha}$ désigneront des coefficients binômiaux qu'il est inutile de préciser.]

(0) $\mathrm{a} Y_{0}=0$;

(1) $|\eta| \mathrm{a} Y_{1}+D_{0} \psi_{1} Y_{0}=0$

(2) $|\eta| \mathrm{a} Y_{2}+D_{0} \psi_{1} Y_{1}+\mathrm{a} D_{*} \psi_{1} Y_{1}+D_{0} \psi_{2} Y_{0}=0$; 
(3) $|\eta| \mathrm{a} Y_{3}+D_{0} \psi_{1} Y_{2}+\mathrm{a} D_{*} \psi_{1} Y_{2}+D_{0} \psi_{2} Y_{1}+\mathrm{a} D_{*} \psi_{2} Y_{1}+D_{0} \psi_{3} Y_{0}$

$$
+\mathrm{a} \sum_{\left|\alpha^{\prime}\right|=2} c_{\alpha^{\prime}} \partial_{\alpha^{\prime}}|\eta| D_{\alpha_{1}^{\prime}} \psi_{1} D_{\alpha_{2}^{\prime}} \psi_{1} Y_{1}=0
$$

(4) $|\eta| \mathrm{a} Y_{4}+D_{0} \psi_{1} Y_{3}+\mathrm{a} D_{*} \psi_{1} Y_{3}+D_{0} \psi_{2} Y_{2}+\mathrm{a} D_{*} \psi_{2} Y_{2}+D_{0} \psi_{3} Y_{1}$

$$
\begin{aligned}
& +\mathrm{a} D_{*} \psi_{3} Y_{1}+D_{0} \psi_{4} Y_{0}+\mathrm{a} \sum_{\left|\alpha^{\prime}\right|=2} c_{\alpha^{\prime}} \partial_{\alpha^{\prime}}|\eta| D_{\alpha_{1}^{\prime}} \psi_{1} D_{\alpha_{2}^{\prime}} \psi_{1} Y_{2} \\
& +\mathrm{a} \sum_{\left|\alpha^{\prime}\right|=2} c_{\alpha^{\prime}} \partial_{\alpha^{\prime}}|\eta| D_{\alpha_{1}^{\prime}} \psi_{1} D_{\alpha_{2}^{\prime}} \psi_{2} Y_{1}=0
\end{aligned}
$$

(5) $|\eta| \mathrm{a} Y_{5}+D_{0} \psi_{1} Y_{4}+\mathrm{a} D_{*} \psi_{1} Y_{4}+D_{0} \psi_{2} Y_{3}+\mathrm{a} D_{*} \psi_{2} Y_{3}+D_{0} \psi_{3} Y_{2}$

$$
\begin{aligned}
& +\mathrm{a} D_{*} \psi_{3} Y_{2}+D_{0} \psi_{4} Y_{1}+\mathrm{a} D_{*} \psi_{4} Y_{1}+D_{0} Y_{0}+b_{0} Y_{0} \\
& +\mathrm{a} \sum_{\left|\alpha^{\prime}\right|=2} c_{\alpha} D_{\alpha^{\prime}}|\eta| D_{\alpha_{1}^{\prime}} \psi_{1} D_{\alpha_{2}^{\prime}} \psi_{1} Y_{3}+\mathrm{a} \sum_{\left|\alpha^{\prime}\right|=2} c_{\alpha^{\prime}} \partial_{\alpha^{\prime}}|\eta| D_{\alpha_{1}^{\prime}} \psi_{1} D_{\alpha_{2}^{\prime}} \psi_{2} Y_{2} \\
& +\mathrm{a} \sum_{\left|\alpha^{\prime}\right|=2} c_{\alpha^{\prime}} D_{\alpha^{\prime}}|\eta| D_{\alpha_{1}^{\prime}} \psi_{1} D_{\alpha_{2}^{\prime}} \psi_{3} Y_{1}+\mathrm{a} \sum_{\left|\alpha^{\prime}\right|=2} c_{\alpha^{\prime}} \partial_{\alpha^{\prime}}|\eta| D_{\alpha_{1}^{\prime}} \psi_{2} D_{\alpha_{2}^{\prime}} \psi_{2} Y_{1} \\
& =0 .
\end{aligned}
$$

Chaque étape s'obtient en augmentant les indices des $Y$ de l'étape précédente d'une unité et en ajoutant les termes nouveaux. Pour alléger l'écriture, nous désignerons par des pointillés les termes suivants le premier terme et déduits par récurrence.

(6) $|\eta| \mathrm{a} Y_{6}+\cdots+\left(D b_{0}\right)_{*}\left(\psi_{1}\right) Y_{0}+\mathrm{a} \sum_{\left|\alpha^{\prime}\right|=2} c_{\alpha^{\prime}} \partial_{\alpha^{\prime}}|\eta| D_{\alpha_{1}^{\prime}} \psi_{1} D_{\alpha_{2}^{\prime}} \psi_{4} Y_{1}$

$$
+\mathrm{a} \sum_{\left|\alpha^{\prime}\right|=2} c_{\alpha^{\prime}} \partial_{\alpha^{\prime}}|\eta| D_{\alpha_{1}^{\prime}} \psi_{2} D_{\alpha_{2}^{\prime}} \psi_{3} Y_{1}=0
$$

(7) $|\eta| \mathrm{a} Y_{7}+\cdots+\left(D b_{0}\right)_{*}\left(\psi_{2}\right) Y_{0}+\sum_{\left|\alpha^{\prime}\right|=2} c_{\alpha^{\prime}} \partial_{\alpha^{\prime}} b_{0} D_{\alpha_{1}^{\prime}} \psi_{1} D_{\alpha_{2}^{\prime}} \psi_{1} Y_{0}$

$$
+\mathrm{a} \sum_{\left|\alpha^{\prime}\right|=2} c_{\alpha^{\prime}} \partial_{\alpha^{\prime}}|\eta| D_{\alpha_{1}^{\prime}} \psi_{2} D_{\alpha_{2}^{\prime}} \psi_{4} Y_{1}+\mathrm{a} \sum_{\left|\alpha^{\prime}\right|=2} c_{\alpha^{\prime}} \partial_{\alpha^{\prime}}|\eta| D_{\alpha_{1}^{\prime}} \psi_{1} D_{\alpha_{2}^{\prime}} Y_{1}=0
$$

(8) $|\eta| \mathrm{a} Y_{8}+\cdots+\left(D b_{0}\right)_{*}\left(\psi_{3}\right) Y_{0}+\sum_{\left|\alpha^{\prime}\right|=2} c_{\alpha^{\prime}} \partial_{\alpha^{\prime}} b_{0} D_{\alpha_{1}^{\prime}} \psi_{1} D_{\alpha_{2}^{\prime}} \psi_{2} Y_{0}$

$$
\begin{aligned}
& +\sum_{\left|\alpha^{\prime}\right|=3} c_{\alpha^{\prime}} \partial_{\alpha^{\prime}} b_{0} D_{\alpha_{1}^{\prime}} \psi_{1} D_{\alpha_{2}^{\prime}} \psi_{1} D_{\alpha_{3}^{\prime}} \psi_{1} Y_{0} \\
& +\mathrm{a} \sum_{\left|\alpha^{\prime}\right|=2} c_{\alpha^{\prime}} \partial_{\alpha^{\prime}}|\eta| D_{\alpha_{1}^{\prime}} \psi_{3} D_{\alpha_{2}^{\prime}} \psi_{4} Y_{1}+\mathrm{a} \sum_{\left|\alpha^{\prime}\right|=2} c_{\alpha^{\prime}} \partial_{\alpha^{\prime}}|\eta| D_{\alpha_{1}^{\prime}} \psi_{2} D_{\alpha_{2}^{\prime}} Y_{1}=0
\end{aligned}
$$

(9) $|\eta| \mathrm{a} Y_{9}+\cdots+\left(\right.$ des termes en $Y_{0}$ et a $Y_{1}$ qu'il sera inutile de préciser $)$ $=0$.

On obtient explicitant chaque étape: 
(0) $Y_{0}^{2}=Y_{0}^{3}=Y_{0}^{4}=Y_{0}^{5}=0$;

(1) $|\eta| Y_{1}^{2}+D_{0} \psi_{1} Y_{0}^{1}=0, \quad Y_{1}^{3}=Y_{1}^{4}=Y_{1}^{5}=0$;

(2) $|\eta| Y_{2}^{2}+\cdots+\left(-\frac{D_{*} \psi_{1} D_{0} \psi_{1}}{|\eta|}+D_{0} \psi_{2}\right) Y_{0}^{1}=0$; $|\eta| Y_{2}^{3}-\frac{\left(D_{0} \psi_{1}\right)^{2}}{|\eta|} Y_{0}^{1}=0, \quad Y_{4}^{2}=Y_{2}^{5}=0$

(3) $|\eta| Y_{3}^{2}+\cdots+\left[\frac{\left(D_{*} \psi_{1}\right)^{2} D_{0} \psi_{1}}{|\eta|^{2}}-\frac{D_{*} \psi_{1} D_{0} \psi_{2}}{|\eta|}-\frac{D_{*} \psi_{2} D_{0} \psi_{1}}{|\eta|}+D_{0} \psi_{3}\right.$ $\left.-\sum_{\left|\alpha^{\prime}\right|=2} c_{\alpha^{\prime}} \partial_{\alpha^{\prime}}|\eta| D_{\alpha_{1}^{\prime}} \psi_{1} D_{\alpha_{2}^{\prime}} \psi_{1} \frac{D_{0} \psi_{1}}{|\eta|}\right] Y_{0}^{1}=0$ $|\eta| Y_{3}^{3}+\cdots+\left(\frac{2\left(D_{0} \psi_{1}\right)^{2} D_{*} \psi_{1}}{|\eta|^{2}}-\frac{2 D_{0} \psi_{1} D_{0} \psi_{2}}{|\eta|}\right) Y_{0}^{1}=0$ $|\eta| Y_{3}^{4}+\frac{\left(D_{0} \psi_{1}\right)^{3}}{|\eta|^{2}} Y_{0}^{1}=0, \quad Y_{3}^{5}=0 ;$

(4) $|\eta| Y_{4}^{2}+\cdots+\left[W\left(\psi_{1}, \psi_{2}, \psi_{3} ; \frac{1}{5}\right)+D_{0} \psi_{4}\right] Y_{0}^{1}=0$.

Notations. $W\left(\psi_{1}, \psi_{2}, \psi_{3} ; \frac{1}{5}\right)$ désigne des termes polynômiaux en $D_{0} \psi_{1}$, $D^{\alpha^{\prime}} \psi_{1}, D_{0} \psi_{2}, D^{\alpha^{\prime}} \psi_{2}, D_{0} \psi_{3}, D^{\alpha^{\prime}} \psi_{3}$ d'ordre $\frac{1}{5}$

$$
\begin{aligned}
& |\eta| Y_{4}^{3}+\cdots+\left[W\left(\psi_{1}, \psi_{2} ; \frac{1}{5}\right)-\frac{2 D_{0} \psi_{3} D_{0} \psi_{1}}{|\eta|}\right] Y_{0}^{1}=0 \\
& |\eta| Y_{4}^{4}+\cdots+\left[\frac{-3\left(D_{0} \psi_{1}\right)^{3} D_{*} \psi_{1}}{|\eta|^{3}}+\frac{3\left(D_{0} \psi_{1}\right)^{2} D_{0} \psi_{2}}{|\eta|^{2}}\right] Y_{0}^{1}=0 \\
& |\eta| Y_{4}^{5}-\frac{\left(D_{0} \psi_{1}\right)^{4}}{|\eta|^{3}} Y_{0}^{1}=0
\end{aligned}
$$

(5) La $5^{\text {ème }}$ ligne donne:

$$
\left[\left(D_{0} \psi_{1}\right)^{5}+b_{01}^{5}|\eta|^{4}\right] Y_{0}^{1}=0
$$

compte tenu du choix de $D_{0} \psi_{1}$, elle est vérifiée sans condition sur $Y_{0}^{1}$

$$
\begin{aligned}
& |\eta| Y_{5}^{2}+\cdots+D_{0} Y_{0}^{1}+W\left(\psi_{1}, \psi_{2}, \psi_{3}, \psi_{4} ; 0\right) Y_{0}^{1}=0 \\
& |\eta| Y_{5}^{3}+\cdots+\left[\frac{-D_{0} \psi_{4} D_{0} \psi_{1}}{|\eta|}+W\left(\psi_{1}, \psi_{2}, \psi_{3} ; 0\right)\right] Y_{0}^{1}=0 \\
& |\eta| Y_{5}^{4}+\cdots+\left[\frac{3\left(D_{0} \psi_{1}\right)^{2} D_{0} \psi_{3}}{|\eta|^{2}}+W\left(\psi_{1}, \psi_{2} ; 0\right)\right] Y_{0}^{1}=0 \\
& |\eta| Y_{5}^{5}+\cdots+\left(\frac{-4\left(D_{0} \psi_{1}\right)^{3} D_{0} \psi_{2}}{|\eta|^{3}}+\frac{4\left(D_{0} \psi_{1}\right)^{4} D_{*} \psi_{1}}{|\eta|^{4}}\right) Y_{0}^{1}=0 .
\end{aligned}
$$


(6) La $5^{\text {ème }}$ ligne donne

$$
\left[5\left(D_{0} \psi_{1}\right)^{4} D_{0} \psi_{2}|\eta|-4\left(D_{0} \psi_{1}\right)^{5} D_{*} \psi_{1}-b_{02}^{5}|\eta|^{4} D_{0} \psi_{1}+|\eta|^{5}\left(D b_{01}^{5}\right)_{*}\left(\psi_{1}\right)\right] Y_{0}^{1}=0 ;
$$

compte tenu du choix de $D_{0} \psi_{2}$, elle est vérifiée sans conditions sur $Y_{0}^{1}$.

$$
\begin{aligned}
& |\eta| Y_{6}^{3}+\cdots+\frac{-2 D_{0} \psi_{1}}{|\eta|} D_{0} Y_{0}^{1}+W\left(\psi_{1}, \psi_{2}, \psi_{3}, \psi_{4} ;-\frac{1}{5}\right) Y_{0}^{1}=0 \\
& |\eta| Y_{6}^{4}+\cdots+\left[\frac{2 D_{0} \psi_{4}\left(D_{0} \psi_{1}\right)^{2}}{|\eta|^{2}}+W\left(\psi_{1}, \psi_{2}, \psi_{3} ;-\frac{1}{5}\right)\right] Y_{0}^{1}=0 \\
& |\eta| Y_{6}^{5}+\cdots+\left[\frac{-4 D_{0} \psi_{3}\left(D_{0} \psi_{1}\right)^{3}}{|\eta|^{3}}+W\left(\psi_{1}, \psi_{2} ;-\frac{1}{5}\right)\right] Y_{0}^{1}=0
\end{aligned}
$$

(7) La $5^{\text {ème }}$ ligne est vérifiée sans condition sur $Y_{0}^{1}$ en choisissant convenablement $D_{0} \psi_{3}$ :

$$
\begin{aligned}
& |\eta| Y_{7}^{4}+\cdots+\frac{3\left(D_{0} \psi_{1}\right)^{2}}{|\eta|^{2}}+D_{0} Y_{0}^{1}+W\left(\psi_{1}, \psi_{2}, \psi_{3}, \psi_{4} ;-\frac{2}{5}\right) Y_{0}^{1}=0 \\
& |\eta| Y_{7}^{5}+\cdots+\left[\frac{-3 D_{0} \psi_{4}\left(D_{0} \psi_{1}\right)^{3}}{|\eta|^{3}}+W\left(\psi_{1}, \psi_{2}, \psi_{3} ;-\frac{2}{5}\right)\right] Y_{0}^{1}=0 .
\end{aligned}
$$

(8) La $5^{\text {ème }}$ ligne est vérifiée sans condition sur $Y_{0}^{1}$ en choisissant convenablement $D_{0} \psi_{4}$

$$
|\eta| Y_{8}^{5}+\cdots-\frac{4\left(D_{0} \psi_{1}\right)^{3}}{|\eta|^{3}} D_{0} Y_{0}^{1}+W\left(\psi_{1}, \psi_{2}, \psi_{3}, \psi_{4} ;-\frac{3}{5}\right) Y_{0}^{1}=0 .
$$

(9) La $5^{\text {ème }}$ ligne donne l'équation de propagation le long des bicaractéristiques:

$$
\frac{5\left(D_{0} \psi_{1}\right)^{4}}{|\eta|^{4}} D_{0} Y_{0}^{1}+W\left(\psi_{1}, \psi_{2}, \psi_{3}, \psi_{4} ;-\frac{4}{5}\right) Y_{0}^{1}=0
$$

L'étude des conditions initiales déterminera complètement $Y_{0}^{1}$ par intégration de l'équation différentielle en $x_{0}$ précédente; on a alors aussi déterminé $Y_{1}^{2}$. L'étape suivante (10) déterminera de même $Y_{1}^{1}$ et ainsi tous les $Y_{1}$ sont déterminés. Par récurrence on obtient de même tous les $Y_{k}$, c'est-à-dire tous les $Y_{k}^{t}$. On impose les conditions initiales:
(0) $\left.\sum_{t} Y_{0}^{t}\right|_{x_{0}=\underline{x}_{0}}=0$
(1) $\left.\sum_{t} Y_{1}^{t}\right|_{x_{0}=\underline{x}_{0}}=0$
(2) $\left.\sum_{t} Y_{2}^{t}\right|_{x_{0}=\underline{x}_{0}}=0$
(3) $\left.\sum_{t} Y_{3}^{t}\right|_{x_{0}=\underline{x}_{0}}=0$
(4) $\left.\sum_{t} Y_{4}^{t}\right|_{x_{0}=\underline{x}_{0}}=I$
(5) $\left.\sum_{t} Y_{5}^{t}\right|_{x_{0}=\underline{x}_{0}}=0, \ldots$ 
En tenant compte de l'étude précédente du système différentiel et des relations entre les $Y_{k}^{t}$, on obtient:
(0) $\left.\sum_{t}\left(Y_{0}^{t}\right)^{1}\right|_{x_{0}=\underline{x}_{0}}=0$
(1) $\left.\sum_{t} D_{0} \psi_{1}^{t}\left(Y_{0}^{t}\right)^{1}\right|_{x_{0}=\underline{x}_{0}}=0$
(2) $\left.\sum_{t}\left(D_{0} \psi_{1}^{t}\right)^{2}\left(Y_{0}^{t}\right)^{1}\right|_{x_{0}=\underline{x}_{0}}=0$
(3) $\left.\sum_{t}\left(D_{0} \psi_{1}^{t}\right)^{3}\left(Y_{0}^{t}\right)^{1}\right|_{x_{0}=\underline{x}_{0}}=0$
(4) $\left.\sum_{t}\left(D_{0} \psi_{0}^{t}\right)^{4}\left(Y_{0}^{t}\right)^{1}\right|_{x_{0}=\underline{x}_{0}}=|\eta|^{4} I^{5}$

On a un système de 5 équations linéaires d'inconnues les 5 symboles $\left.\left(Y_{0}^{t}\right)^{1}\right|_{x_{0}=\underline{x}_{0}}$; le déterminant du système est de Vandermonde non nul; on obtient les données $\left.\left(Y_{0}^{t}\right)^{1}\right|_{x_{0}=\underline{x}_{0}}$ et ainsi de suite tous les symboles analytiques $Y_{\tilde{f}^{t}}^{t}$ avec les majorations usuelles convenables. On a donc finalement les $\tilde{f}_{j}$, les $\tilde{F}_{j}$ et la paramétrixe $F$.

1.2. Cas $(\mathbf{L G})_{4 / 3}$. On cherche $f$ sous la forme:

$$
\begin{aligned}
f & =\sum_{1 \leq t \leq 4} f_{1}^{t}+f_{2}, \\
f_{1}^{t} & =e^{\psi_{1}^{t}+\psi_{2}^{t}+\psi_{3}^{t}}\left(Y_{0}^{t}+\cdots+Y_{k}^{t}+\cdots\right)
\end{aligned}
$$

les 4 phases complexes distinctes $\psi_{1}^{t}, 1 \leq t \leq 4$, vérifient:

$$
\left(D_{0} \psi_{1}^{t}\right)^{4}=-b_{02}^{5}|\eta|^{3} \neq 0 ;\left.\quad \psi_{1}^{t}\right|_{x_{0}=\underline{x}_{0}}=0 ;
$$

chaque $\psi_{1}^{t}$ est d'ordre: $3 / 4$; les 4 phases $\psi_{2}^{t}$ sont d'ordre $1 / 2$ et les 4 phases $\psi_{3}^{t}$ sont d'ordre $1 / 4$; elles sont déterminées comme dans le cas précédent. $Y_{k}^{t}$ est d'ordre $\frac{4-k}{4}$

$$
f_{2}=Z_{0}+\cdots+Z_{k}+\cdots
$$

$Z_{k}$ est d'ordre: $\frac{4-k}{4} . \quad f_{2}$ s'interprète comme un terme complémentaire dû au deuxième segment horizontal du diagramme $N$, comme dans le cas des opérateurs à coefficients constants.

Pour réaliser $h \circ f=0$, on annule d'abord la partie de $f$ dûe à chaque $f_{1}^{t}$. On obtient comme précédemment un système différentiel pour les $Y_{k}^{t}$ :

(0) $\quad \mathrm{a} Y_{0}=0$

(1) $|\eta| \mathrm{a} Y_{1}+D_{0} \psi_{1} Y_{0}=0$

(2) $|\eta| \mathrm{a} Y_{2}+\cdots+\mathrm{a} D_{*} \psi_{1} Y_{1}+D_{0} \psi_{2} Y_{0}=0$ 
(3) $|\eta| \mathrm{a} Y_{3}+\cdots+D_{0} \psi_{3} Y_{0}+\mathrm{a} W\left(\psi_{1} ; \frac{1}{2}\right) Y_{1}=0$

$W$ est polynomial en $D_{0} \psi_{1}$ d'ordre $1 / 2$

(4) $|\eta| \mathrm{a} Y_{4}+\cdots+D_{0} Y_{0}+b_{0} Y_{0}+\mathrm{a} W\left(\psi_{1}, \psi_{2} ; \frac{1}{4}\right) Y_{1}=0$

(5) $|\eta| \mathrm{a} Y_{5}+\cdots+\left(D b_{0}\right)_{*}\left(\psi_{1}\right) Y_{0}+\mathrm{a} W\left(\psi_{1}, \psi_{2}, \psi_{3} ; 0\right) Y_{1}=0$

(6) $|\eta| \mathrm{a} Y_{6}+\cdots+\left(D b_{0}\right)_{*}\left(\psi_{2}\right) Y_{0}+W\left(\psi_{1},-\frac{1}{2}\right) Y_{0}+\mathrm{a} W\left(\psi_{1} ; \frac{1}{4}\right) D_{*} Y_{1}=0$

(7) $|\eta| \mathrm{a} Y_{7}+\cdots+\left(D b_{0}\right)_{*}\left(\psi_{3}\right) Y_{0}+W\left(\psi_{1}, \psi_{2} ;-\frac{3}{4}\right) Y_{0}$

$$
+\mathrm{aW}\left(\psi_{1}, \psi_{2} ;-\frac{1}{2}\right) D_{*} Y_{1}=0
$$

(8) $|\eta| \mathrm{a} Y_{8}+\cdots+\left(D b_{0}\right)_{*}(Y)_{0}+b_{1} Y_{0}+W\left(\psi_{1}, \psi_{2}, \psi_{3} ;-1\right) Y_{0}$

$$
+\mathrm{aW}\left(\psi_{1}, \psi_{2}, \psi_{3} ;-\frac{3}{4}\right) D_{*} Y_{1}=0
$$

Les $W$ commencent par des termes en $\partial_{\alpha^{\prime}} b_{0},\left|\alpha^{\prime}\right|=2$. On obtient, en n'indiquant que les termes les plus significatifs:

(0) $Y_{0}^{2}=Y_{0}^{3}=Y_{0}^{4}=Y_{0}^{5}=0$;

(1) $|\eta| Y_{1}^{2}+D_{0} \psi_{1} Y_{0}^{1}=0, \quad Y_{1}^{3}=Y_{1}^{4}=Y_{1}^{5}=0$;

(2) $|\eta| Y_{2}^{3}-\frac{\left(D_{0} \psi_{1}\right)^{2}}{|\eta|} Y_{0}^{1}=0, \quad Y_{2}^{4}=Y_{2}^{5}=0$

(3) $|\eta| Y_{3}^{4}+\frac{\left(D_{0} \psi_{1}\right)^{3}}{|\eta|} Y_{0}^{1}=0, \quad Y_{3}^{5}=0$;

(4) $|\eta| Y_{4}^{2}+\cdots+D_{0} Y_{0}^{1}+W\left(\psi_{1}, \psi_{2} ; 0\right) Y_{0}^{1}=0 ; \quad|\eta| Y_{4}^{5}-\frac{\left(D_{0} \psi_{1}\right)^{4}}{|\eta|} Y_{0}^{1}=0$.

(5) La $5^{\text {ème }}$ ligne est réalisée, sans condition sur $Y_{0}^{1}$, compte tenu du choix de $\psi_{1}$ :

$$
\begin{gathered}
{\left[\left(D_{0} \psi_{1}\right)^{5}-b_{02}^{5}|\eta|^{3} D_{0} \psi_{1}\right] Y_{0}^{1}=0} \\
|\eta| Y_{5}^{3}+\cdots-2 \frac{D_{0} \psi_{1}}{|\eta|} D_{0} Y_{0}^{1}+W\left(\psi_{1}, \psi_{2}, \psi_{3} ;-\frac{1}{4}\right) Y_{0}^{1}=0
\end{gathered}
$$

(6) La $5^{\text {ème }}$ ligne est réalisée, sans condition sur $Y_{0}^{1}$, compte tenu du choix de $\psi_{2}$ :

$$
\begin{gathered}
{\left[\left(5\left(D_{0} \psi_{1}\right)^{4}-b_{02}^{5}|\eta|^{3}\right) D_{0} \psi_{2}+W\left(\psi_{1} ; \frac{7}{2}\right)\right] Y_{0}^{1}=0} \\
|\eta| Y_{6}^{4}+\cdots+\frac{3\left(D_{0} \psi_{1}\right)^{2}}{|\eta|^{2}} D_{0} Y_{0}^{1}+W\left(\psi_{1}, \psi_{2}, \psi_{3} ;-\frac{1}{2}\right) Y_{0}^{1}=0
\end{gathered}
$$


(7) La $5^{\text {ème }}$ ligne est réalisée, sans condition sur $Y_{0}^{1}$, compte tenu du choix de $\psi_{3}$ :

$$
|\eta| Y_{7}^{5}-4 \frac{\left(D_{0} \psi_{1}\right)^{3}}{|\eta|^{3}} D_{0} Y_{0}^{1}+W\left(\psi_{1}, \psi_{2}, \psi_{3} ;-\frac{3}{4}\right) Y_{0}^{1}=0
$$

(8) La $5^{\text {ème }}$ ligne donne:

$$
4\left(D_{0} \psi_{1}\right)^{4} D_{0} Y_{0}^{1}+W\left(\psi_{1}, \psi_{2}, \psi_{3} ; 3\right) Y_{0}^{1}=0
$$

L'étude des conditions initiales déterminera les $\left.Y_{0}^{1}\right|_{x_{0}=\underline{x}_{0}}$, et par suite les $Y_{0}^{1}$ et tous les $Y_{k}$.

On annule le terme en $f_{2}$; on obtient le système différentiel:
(0) $\mathrm{a} Z_{0}=0$
(1) $\mathrm{a} Z_{1}=0$;
(2) $a Z_{2}=0$;
(3) $\mathrm{a} Z_{3}=0$
(4) $|\eta| \mathrm{a} Z_{4}+D_{0} Z_{0}+b_{0} Z_{0}=0$
(5) $|\eta| \mathrm{a} Z_{5}+D_{0} Z_{1}+b_{0} Z_{1}=0$;
(6) $|\eta| \mathrm{a} Z_{6}+D_{0} Z_{2}+b_{0} Z_{2}=0$
(7) $|\eta| \mathrm{a} Z_{7}+D_{0} Z_{3}+b_{0} Z_{3}=0$;
(8) $|\eta| \mathrm{a} Z_{8}+D_{0} Z_{4}+\mathrm{a} D_{*} Z_{4}+b_{0} Z_{4}+b_{1} Z_{0}=0$.

On obtient:
(0) $Z_{0}^{2}=Z_{0}^{3}=Z_{0}^{4}=Z_{0}^{5}=0$;
(1) $Z_{1}^{2}=Z_{1}^{3}=Z_{1}^{4}=Z_{1}^{5}=0$;
(2) $Z_{2}^{2}=Z_{2}^{3}=Z_{2}^{4}=Z_{2}^{5}=0$;
(3) $Z_{3}^{2}=Z_{3}^{3}=Z_{3}^{4}=Z_{3}^{5}=0$;
(4) $|\eta| Z_{4}^{2}+D_{0} Z_{0}^{1}=0 ; \quad Z_{4}^{2}=Z_{4}^{4}=Z_{4}^{5}=0$
(5) (6) (7).

(8) La $5^{\text {ème }}$ ligne donne:

$$
-b_{02}^{5} D_{0} Z_{0}^{1}+|\eta| b_{11}^{5} Z_{0}^{1}=0 .
$$

L'étude des conditions initiales déterminera $\left.Z_{0}^{1}\right|_{x_{0}=\underline{x}_{0}}$, et par suite $Z_{0}^{1}$ et tous les $Z_{k}$.

On impose les conditions initiales:
(0) $\sum_{t} Y_{0}^{t}\left|+Z_{0}\right|=0$;
(1) $\sum_{t} Y_{1}^{t}\left|+Z_{1}\right|=0$
(2) $\sum_{t} Y_{2}^{t}\left|+Z_{2}\right|=0$
(3) $\sum_{t} Y_{3}^{t}\left|+Z_{3}\right|=0$
(4) $\sum_{t} Y_{4}^{t}\left|+Z_{4}\right|=I$

Notations. Pour simplifier les notations on a remplacé $\left.\right|_{x_{0}=\underline{x}_{0}}$ par $\mid$; on fera de même dans la suite. 
On en déduit:
(0) $\sum_{t} Y_{0}^{t 1}\left|+Z_{0}^{1}\right|=0$;
(1) $\sum_{t} D_{0} \psi_{1} Y_{0}^{t 1} \mid=0$
(2) $\sum_{t}\left(D_{0} \psi_{1}^{t}\right)^{2} Y_{0}^{t 1} \mid=0$;
(3) $\sum_{t}\left(D_{0} \psi_{1}\right)^{2} Y_{0}^{t 1} \mid=0$;
(4) $-\frac{b_{02}^{5}}{|\eta|} \sum_{t} Y_{0}^{t 1} \mid=I^{5}$;

on obtient donc les $Y_{0}^{t 1} \mid$ et $Z_{0}^{1} \mid$, d'où tous les coefficients.

1.3. Cas $(\mathbf{L G})_{3 / 2}$. On cherche $f$ sous la forme

$$
\begin{aligned}
f & =\sum_{1 \leq t \leq 3} f_{1}^{t}+f_{2}, \\
f_{1}^{t} & =e^{\psi_{1}^{t}+\psi_{2}^{t}}\left(Y_{0}^{t}+\cdots+Y_{k}^{t}+\cdots\right) ;
\end{aligned}
$$

Les 3 phases complexes distinctes $\psi_{1}^{t}, 1 \leq t \leq 3$, vérifient:

$$
\left(D_{0} \psi_{1}^{t}\right)^{3}=-|\eta|^{2} b_{03}^{5} \neq 0 ; \quad \psi_{1}^{t} \mid=0 ;
$$

chaque $\psi_{1}^{t}$ est d'ordre $2 / 3$; les 3 phases $\psi_{2}^{t}$ sont d'ordre $1 / 3$ et sont déterminées comme précédemment. Le diagramme $N$ est formé des points: $0,(3,2),(5,3)$. Les termes en $f_{1}^{t}$ correspondent au premier segment: $0,(3,2)$.

a) $\quad b_{11}^{5} \neq 0 . \quad Y_{k}^{t}$ est d'ordre $\frac{8-k}{6} . f_{2}$ sera cherché de la forme:

avec:

$$
f_{2}=\sum_{1 \leq t^{\prime} \leq 2} f_{2}^{t^{\prime}}
$$

$$
f_{2}^{t^{\prime}}=e^{\chi^{t^{\prime}}}\left[Z_{0}^{t^{\prime}}+\cdots+Z_{0}^{t^{\prime}}+\cdots\right]
$$

les 2 phases complexes distinctes $\chi^{t^{\prime}} ; 1 \leq t^{\prime} \leq 2$ vérifient:

$$
\left(D_{0} \chi^{t^{\prime}}\right)^{2}=-\frac{b_{11}^{5}|\eta|^{2}}{b_{03}^{5}} ; \quad \chi^{t^{\prime}} \mid=0 ;
$$

chaque $\chi^{t^{\prime}}$ est d'ordre: $1 / 2 ; Z_{k}^{t^{\prime}}$ est d'ordre $\frac{9-k}{6}$.

Ces termes supplémentaires correspondent au 2ème segment de $N$, défini par les points $(3,2)$ et $(5,3)$, de pente: $1 / 2$.

b) $\quad b_{11}^{5}=0$. On supprime le point $(5,3)$ dans $N$ et le diagramme $N$ se réduit à $0,(3,2),(4,2),(5,2)$; le deuxième segment contenant les points: $(3,2),(4,2),(5,2)$ est horizontal; $Y_{k}^{t}$ est d'ordre: $\frac{4-k}{3} . f_{2}$ sera cherché de la forme:

$$
f_{2}=Z_{0}+\cdots+Z_{k}+\cdots
$$


$Z_{k}$ est d'ordre: $\frac{6-k}{3}$.

On étudie le cas a).

Le système en $Y_{k}^{t}$ s'écrit:
(0) $\mathrm{a} Y_{0}=0$
(1) $\mathrm{a} Y_{1}=0$
(2) $|\eta| \mathrm{a} Y_{2}+D_{0} \psi_{1} Y_{0}=0$
(3) $|\eta| \mathrm{a} Y_{3}+\cdots=0$
(4) $|\eta| \mathrm{a} Y_{4}+\cdots+D_{0} \psi_{2} Y_{0}=0$
(5) $|\eta| \mathrm{a} Y_{5}+\cdots=0$
(6) $|\eta| \mathrm{a} Y_{6}+\cdots+D_{0} Y_{0}+b_{0} Y_{0}+\mathrm{a} W\left(\psi_{1} ; \frac{1}{3}\right) Y_{2}=0$;
(7) $|\eta| \mathrm{a} Y_{7}+\cdots+\mathrm{a} W\left(\psi_{1}, \psi_{2} ;-\frac{1}{6}\right) Y_{2}=0$.

Ensuite à chaque étape, on obtient les termes de récurrence et des termes supplémentaires; nous indiquerons seulement les termes les plus significatifs et les équations utiles pour démarrer la récurrence:

(8) $|\eta| \mathrm{a} Y_{8}+\cdots+\mathrm{a} D_{*} Y_{2}+\left(D b_{0}\right)_{*}\left(\psi_{1}\right) Y_{0}=0$;

(9) $|\eta| \mathrm{a} Y_{9}+\cdots=0 ; \quad$ (10) $|\eta| \mathrm{a} Y_{10}+\cdots+\left(D b_{0}\right)_{*}\left(\psi_{2}\right) Y_{0}=0$;

(11) $|\eta| \mathrm{a} Y_{11}+\cdots=0$;

(12) $|\eta| \mathrm{a} Y_{12}+\cdots+b_{1} Y_{0}+\left(D b_{0}\right)_{*}\left(Y_{0}\right)=0$;

(13) $|\eta| \mathrm{a} Y_{13}+\cdots=0$;

(14) $|\eta| \mathrm{a} Y_{14}+\cdots=0$.

Les calculs sont analogues à ceux du cas précédent
(0) $Y_{0}^{2}=Y_{0}^{3}=Y_{0}^{4}=Y_{0}^{5}=0 ; \quad \ldots$;
(2) $|\eta| Y_{2}^{2}+D_{0} \psi_{1} Y_{0}^{1}=0 ; \quad \ldots$;
(4) $|\eta| Y_{4}^{3}-\frac{\left(D_{0} \psi_{1}\right)^{2}}{|\eta|} Y_{0}^{1}=0 ; \quad \ldots$;
(6) $|\eta| Y_{6}^{4}+\frac{\left(D_{0} \psi_{1}\right)^{3}}{|\eta|^{2}} Y_{0}^{1}=0 ; \quad \ldots$;
(8) $|\eta| Y_{8}^{3}+\cdots-\frac{D_{0} \psi_{1}}{|\eta|} D_{0} Y_{0}^{1}+\cdots=0$;
$|\eta| Y_{8}^{5}-\frac{\left(D_{0} \psi_{1}\right)^{4}}{|\eta|^{3}} Y_{0}^{1}=0 ; \quad \ldots$

A l'étape (10), à la $5^{\text {ème }}$ ligne on obtient:

$$
\left[\frac{\left(D_{0} \psi_{1}\right)^{5}}{|\eta|^{4}}+b_{03}^{5} \frac{\left(D_{0} \psi_{1}\right)^{2}}{|\eta|^{2}}\right] Y_{0}^{3}=0
$$

et:

$$
|\eta| Y_{10}^{4}+\cdots+2 \frac{\left(D_{0} \psi_{1}\right)^{2}}{|\eta|^{2}} D_{0} Y_{0}^{1}+W\left(\psi_{1}, \psi_{2} ;-\frac{2}{3}\right) Y_{0}^{1}=0 ; \quad \ldots
$$

à l'étape (12), compte tenu du choix de $\psi_{2}$, la $5^{\text {ème }}$ ligne est réalisée sous condition sur $Y_{0}^{1}$; on a aussi

$$
|\eta| Y_{12}^{5} \cdots+3 \frac{\left(D_{0} \psi_{1}\right)^{3}}{|\eta|^{3}} D_{0} Y_{0}^{1}+W\left(\psi_{1}, \psi_{2},-1\right) Y_{0}^{1}=0
$$


à l'étape (14), la $5^{\text {ème }}$ ligne donne:

$$
3\left(D_{0} \psi_{1}\right)^{4} D_{0} Y_{0}^{1}+W\left(\psi_{1}, \psi_{2} ; \frac{8}{3}\right) Y_{0}^{1}=0
$$

Il suffira donc de connaître les valeurs des $\left.\left(Y_{0}^{t}\right)^{1}\right|_{x_{0}=\underline{x}_{0}}$. On a aussi:

(0) (1) (2) $\quad \mathrm{a} Z_{0}=\mathrm{a} Z_{1}=\mathrm{a} Z_{2}=0$

(3) $|\eta| \mathrm{a} Z_{3}+D_{0} \chi Z_{0}=0$

(4) $|\eta| \mathrm{a} Z_{4}+D_{0} \chi Z_{1}=0$

(5) $|\eta| \mathrm{a} Z_{5}+D_{0} \chi Z_{2}=0$

(6) $|\eta| \mathrm{a} Z_{6}+D_{0} \chi Z_{3}+\mathrm{a} D_{*} \chi Z_{3}+D_{0} Z_{0}+b_{0} Z_{0}=0$

à l'étape (12) apparaît $b_{1} Z_{0}+\left(b_{0}\right)_{*}\left(Z_{0}\right), \ldots$.

On a:

(0) (1) (2) $Z_{0}^{2}=Z_{0}^{3}=Z_{0}^{4}=Z_{0}^{5}=\cdots=Z_{2}^{2}=\cdots=Z_{2}^{5}=0$;

(3) $|\eta| Z_{3}^{2}+D_{0} \chi Z_{0}^{1}=0 ; \quad \ldots$;

(6) $|\eta| Z_{6}^{2}+\cdots+D_{0} Z_{0}^{1}-\frac{D_{*} \chi D_{0} \chi}{|\eta|} Z_{0}^{1}=0, \quad|\eta| Z_{6}^{3}-\frac{\left(D_{0} \chi\right)^{2}}{|\eta|} Z_{0}^{1}=0 ; \ldots$;

(9) $|\eta| Z_{9}^{3}+\cdots-\frac{2\left(D_{0} \chi\right)^{2}}{|\eta|} D_{0} Z_{0}^{1}+W(\chi ; 0) Z_{0}^{1}=0,|\eta| Z_{9}^{4}+\frac{\left(D_{0} \chi\right)^{3}}{|\eta|^{2}}=0 ; \ldots$;

à la 12 ème étape la $5^{\text {ème }}$ ligne donne:

$$
\left[b_{03}^{5} \frac{\left(D_{0} \chi\right)^{2}}{|\eta|^{2}}+b_{11}^{5}\right] Z_{0}^{1}=0
$$

qui est réalisée sans condition sur $Z_{0}^{1}$ par le choix de la phase secondaire $\chi$; à la $15^{\text {ème }}$ étape, la $5^{\text {ème }}$ ligne donne:

$$
\frac{2 b_{03}^{5}\left(D_{0} \chi\right)^{2}}{|\eta|^{2}} D_{0} Z_{0}^{1}+W(\chi ;-1) Z_{0}^{1}=0
$$

Il suffit donc de connaître les valeurs des $\left.\left(Z_{0}^{t^{\prime}}\right)^{1}\right|_{x_{0}=\underline{x}_{0}}$.

On impose les conditions initiales:
(0) $\quad \sum_{t^{\prime}} Z_{0}^{t^{\prime}} \mid=0$;
(1) $\sum_{t} Y_{0}^{t}\left|+\sum_{t^{\prime}} Z_{1}^{t^{\prime}}\right|=0$
(2) $\quad \sum_{t} Y_{1}^{t}+\sum_{t^{\prime}} Z_{2}^{t^{\prime}} \mid=0$;
(3) $\sum_{t} Y_{2}^{t}\left|+\sum_{t^{\prime}} Z_{3}^{t^{\prime}}\right|=0 ; \quad \ldots$;
(5) $\quad \sum_{t} Y_{4}^{t}\left|+\sum_{t^{\prime}} Z_{5}^{t^{\prime}}\right|=0 ; \quad \ldots$;
(9) $\sum_{t} Y_{8}^{t}\left|+\sum_{t^{\prime}} Z_{9}^{t^{\prime}}\right|=I$
(10) $\quad \sum_{t} Y_{9}^{t}\left|+\sum_{t^{\prime}} Z_{10}^{t^{\prime}}\right|=0$; 
On a:

$$
\text { (0) } \quad \sum_{t^{\prime}} Z_{0}^{t^{\prime} 1} \mid=0
$$

à l'aide de (3):

$$
\sum_{t} D_{0} \psi_{1}^{t} Y_{0}^{t 1}\left|+\sum_{t^{\prime}} D_{0} \chi^{t^{\prime}} Z_{0}^{t^{\prime} 1}\right|=0
$$

à l'aide de (5):

$$
\sum_{t}\left(D_{0} \psi_{1}^{t}\right)^{2} Y_{0}^{t 1} \mid=0
$$

à l'aide de (7): comme $b_{03}^{5} \neq 0$,

$$
\sum_{t} Y_{0}^{t 1} \mid=0
$$

à l'aide de (9), on a

$$
-\frac{b_{03}^{5}}{|\eta|^{2}} \sum_{t} D_{0} \psi_{1}^{t} Y_{0}^{t 1} \mid=I^{5}
$$

On a ainsi par un Vandermonde, les $Y_{0}^{t 1} \mid$; on a aussi:

$$
\sum_{t^{\prime}} D_{0} \chi^{t^{\prime}} Z_{0}^{t^{\prime} 1} \mid=\frac{|\eta|^{2}}{b_{03}^{5} \mid} I^{5}
$$

d'où, à l'aide d'un autre Vandermonde, les $Z_{0}^{t^{\prime} 1} \mid$. Finalement on a tous les $Y$ et $Z$.

b) $\quad b_{11}^{5}=0 . \quad$ Nous ne donnons que les termes les plus significatifs
(0) $\mathrm{a} Y_{0}=0$;
(1) $|\eta| \mathrm{a} Y_{1}+D_{0} \psi_{1} Y_{0}=0$
(2) $|\eta| \mathrm{a} Y_{2}+\cdots+D_{0} \psi_{2} Y_{0}=0$
(3) $|\eta| \mathrm{a} Y_{3}+\cdots+D_{0} Y_{0}+b_{0} Y_{0}=0$;
(4) $|\eta| \mathrm{a} Y_{4}+\cdots=0$
(5) $|\eta| \mathrm{a} Y_{5}+\cdots=0$
(6) $|\eta| \mathrm{a} Y_{6}+\cdots=0$
(7) $|\eta| \mathrm{a} Y_{7}+\cdots=0$.

On a:

(0) $Y_{0}^{2}=Y_{0}^{3}=Y_{0}^{4}=Y_{0}^{5}=0$;

(1) $Y_{1}^{2}+D_{0} \psi_{1} Y_{0}^{1}=0, \quad Y_{1}^{3}=Y_{1}^{4}=Y_{1}^{5}=0$;

(2) $|\eta| Y_{2}^{2}+\left[D_{0} \psi_{2}+W\left(\psi_{1} ; \frac{1}{3}\right)\right] Y_{0}^{1}=0 ;$

$$
|\eta| Y_{2}^{3}-\frac{\left(D_{0} \psi_{1}\right)^{2}}{|\eta|} Y_{0}^{1}=0, \quad Y_{2}^{4}=Y_{2}^{5}=0
$$

(3) $|\eta| Y_{3}^{2}+\cdots+D_{0} Y_{0}^{1}+W\left(\psi_{1}, \psi_{2} ; 0\right) Y_{0}^{1}=0$; 


$$
\begin{aligned}
& |\eta| Y_{3}^{4}+\cdots+\frac{\left(D_{0} \psi_{1}\right)^{3}}{|\eta|^{2}} Y_{0}^{1}=0 ; \quad Y_{3}^{5}=0 ; \\
& |\eta| Y_{4}^{3}+\cdots-\frac{2 D_{0} \psi_{1}}{|\eta|} D_{0} Y_{0}^{1}+W\left(\psi_{1}, \psi_{2} ;-\frac{1}{3}\right) Y_{0}^{1}=0 \\
& |\eta| Y_{4}^{4}+\cdots+\left[\frac{3\left(D_{0} \psi_{1}\right)^{2} D_{0} \psi_{2}}{|\eta|^{2}}\right]+W\left(\psi_{1} ;-\frac{1}{3}\right) Y_{0}^{1}=0 \\
& |\eta| Y_{4}^{5}-\frac{\left(D_{0} \psi_{1}\right)^{4}}{|\eta|^{3}} Y_{0}^{1}=0 .
\end{aligned}
$$

(5) La $5^{\text {ème }}$ ligne laisse $Y_{0}^{1}$ libre compte tenu du choix de $\psi_{1}$

$$
\begin{aligned}
& |\eta| Y_{5}^{4}+\cdots+\frac{3\left(D_{0} \psi_{1}\right)^{2}}{|\eta|^{2}} D_{0} Y_{0}^{1}+W\left(\psi_{1}, \psi_{2} ;-\frac{2}{3}\right) Y_{0}^{1}=0 \\
& |\eta| Y_{5}^{5}+\cdots+\left[-\frac{4\left(D_{0} \psi_{1}\right)^{3} b_{0} \psi_{2}}{|\eta|^{3}}+W\left(\psi_{1} ;-\frac{2}{3}\right)\right] Y_{0}^{1}=0 .
\end{aligned}
$$

(6) La $5^{\text {ème }}$ ligne laisse $Y_{0}^{1}$ libre compte tenu du choix de $\psi_{2}$

$$
|\eta| Y_{6}^{5}-4 \frac{\left(D_{0} \psi_{1}\right)^{3}}{|\eta|^{3}} D_{0} Y_{0}^{1}+W\left(\psi_{1}, \psi_{2} ;-1\right) Y_{0}^{1}=0
$$

(7) Le $5^{\text {ème }}$ ligne donne:

$$
3\left(D_{0} \psi_{1}\right)^{3} D_{0} Y_{0}^{1}+W\left(\psi_{1}, \psi_{2} ; 2\right) Y_{0}^{1}=0 .
$$

On a aussi:

(0) $\quad \mathrm{a} Z_{0}=0 ; \quad$ (1) $\quad \mathrm{a} Z_{1}=0 ; \quad$ (2) $\quad \mathrm{a} Z_{2}=0$;

(3) $|\eta| \mathrm{a} Z_{3}+D_{0} Z_{0}+b_{0} Z_{0}=0 ; \quad$ (4) $\ldots ; \quad$ (5) $\quad \ldots$;

(6) $|\eta| \mathrm{a} Z_{6}+\cdots+b_{1} Z_{0}+\left(D b_{0}\right)_{*}\left(Z_{0}\right)=0$;

(7) $\ldots$; (8) $\ldots$; (9) $|\eta| \mathrm{a} Z_{9}+\cdots+b_{2} Z_{0}+\left(D b_{1}\right)_{*}\left(Z_{0}\right)+\cdots=0$.

On a:

(0) $\quad Z_{0}^{2}=Z_{0}^{3}=Z_{0}^{4}=Z_{0}^{5}=0 ; \quad \ldots ; \quad$ (3) $|\eta| Z_{3}^{2}+D_{0} Z_{0}^{1}=0 ; \quad \ldots$;

(6) $|\eta| Z_{6}^{3}-\frac{1}{|\eta|} D_{0}^{2} Z_{0}^{1}=0 ; \quad \ldots$

(9) $\quad b_{03}^{5} D_{0}^{2} Z_{0}^{1}-b_{12}^{5}|\eta| D_{0} Z_{0}^{1}+b_{21}^{5}|\eta|^{2} Z_{0}^{1}=0$.

On impose les conditions initiales:
(0) $Z_{0} \mid=0$;
(1) $Z_{1} \mid=0$
(2) $\quad \sum_{t} Y_{0}^{t}\left|+Z_{2}\right|=0$;
(3) $\sum_{t} Y_{1}^{t}\left|+Z_{3}\right|=0$;
(4) $\ldots$;
(5) $\ldots$;
(6) $\sum_{t} Y_{4}^{t}\left|+Z_{6}\right|=I$; 
On en déduit:
(0) $\quad Z_{0}^{1} \mid=0 ; \quad \ldots$;
(3) $\sum_{t} D_{0} \psi_{1}^{t} Y_{0}^{t 1}\left|+D_{0} Z_{0}^{1}\right|=0$;
(4) $\sum_{t}\left(D_{0} \psi_{1}^{t}\right)^{2} Y_{0}^{t 1} \mid=0$;
(5) $\sum_{t} Y_{0}^{t 1} \mid=0$
(6) $\left.\quad b_{03}^{5} \sum_{t} D_{0} \psi_{1}^{t} Y_{0}^{t 1}|=-| \eta\right|^{2} I^{5}$;

on obtient les $Y_{0}^{t 1}\left|, D_{0} Z_{0}^{1}\right|$ et par suite tous les coefficients.

1.4. Cas $(\mathbf{L G})_{5 / 3}$. On cherche $f$ sous la forme:

$$
\begin{gathered}
f=\sum_{1 \leq t \leq 5} e^{\psi_{1}^{t}+\psi_{2}^{t}+\psi_{3}^{t}}\left(Y_{0}^{t}+\cdots+Y_{k}^{t}+\cdots\right) \\
\left(D_{0} \psi_{1}^{t}\right)^{5}=-|\eta|^{3} b_{11}^{5} \neq 0 ; \quad \psi_{1}^{t} \mid \equiv 0,
\end{gathered}
$$

$\psi_{1}^{t}$ est d'ordre $3 / 5 ; \psi_{2}^{t}$ et $\psi_{3}^{t}$ sont d'ordre $2 / 5$ et $1 / 5$ respectivement et sont déterminés comme précédemment; $Y_{k}^{t}$ est d'ordre $\frac{8-k}{5}$.

On écrit les systèmes en $Y$ :

$$
\text { (0) (1) } \quad \ldots ; \quad(13) ;
$$

on obtient:

$$
\begin{array}{ll}
Y_{0}^{2}=Y_{0}^{3}=Y_{0}^{4}=Y_{0}^{5}=0 ; & |\eta| Y_{2}^{2}+D_{0} \psi_{1} Y_{0}^{1}=0 ; \\
|\eta| Y_{4}^{3}-\frac{\left(D_{0} \psi_{1}\right)^{2}}{|\eta|} Y_{0}^{1}=0 ; & |\eta| Y_{5}^{2}+\cdots+D_{0} Y_{0}^{1}+W\left(\psi_{1}, \psi_{2} ; 0\right) Y_{0}^{1}=0 ; \\
|\eta| Y_{6}^{4}+\frac{\left(D_{0} \psi_{1}\right)^{3}}{|\eta|^{2}} Y_{0}^{1}=0 ; & |\eta| Y_{7}^{3}+\cdots-\frac{2 D_{0} \psi_{1} D_{0} Y_{0}^{1}}{|\eta|}+W\left(\psi_{1}, \psi_{2}, \psi_{3} ;-\frac{2}{5}\right) Y_{0}^{1}=0 \\
\cdots ; & \\
|\eta| Y_{8}^{5}-\frac{\left(D_{0} \psi_{1}\right)^{4}}{|\eta|^{3}} Y_{0}^{1}=0 ; \quad|\eta| Y_{9}^{4}+3 \frac{\left(D_{0} \psi_{1}\right)^{2}}{|\eta|^{2}} D_{0} Y_{0}^{1}+W\left(\psi_{1}, \psi_{2}, \psi_{3} ;-\frac{4}{5}\right) Y_{0}^{1}=0
\end{array}
$$

...; à la 10 ème étape, on a:

$$
\left[\frac{\left(D_{0} \psi_{1}\right)^{5}}{|\eta|^{3}}\right]+b_{11}^{5} Y_{0}^{1}=0
$$

à la 11ème étape:

$$
|\eta| Y_{11}^{5}+\cdots-4 \frac{\left(D_{0} \psi_{1}\right)^{3}}{|\eta|^{3}} D_{0} Y_{0}^{1}+W\left(\psi_{1}, \psi_{2}, \psi_{3} ;-\frac{6}{5}\right) Y_{0}^{1}=0
$$


et on détermine aussi $D_{0} \psi_{2}$;

à la 12ème étape on détermine $D_{0} \psi_{3}$;

à la 13ème étape, on obtient:

$$
5\left(D_{0} \psi_{1}\right)^{4} D_{0} Y_{0}^{1}+W\left(\psi_{1}, \psi_{2}, \psi_{3} ; \frac{12}{5}\right) Y_{0}^{1}=0 .
$$

Il reste à déterminer les $Y_{0}^{1} \mid$; on les obtient aisément en imposant les conditions initiales:

$$
\sum_{t} Y_{0}^{t}\left|=\sum_{t} Y_{1}^{t}\right|=\sum_{t} Y_{2}^{t}\left|=\cdots=\sum_{t} Y_{7}^{t}\right|=0 ; \quad \sum_{t} Y_{8}^{t} \mid=I, \quad \cdots
$$

car on obtient alors:

$$
\begin{aligned}
& \sum_{t} Y_{0}^{t 1}\left|=0 ; \quad \sum_{t} D_{0} \psi_{1}^{t} Y_{0}^{t 1}\right|=0 ; \quad \sum_{t}\left(D_{0} \psi_{1}^{t}\right)^{2} Y_{0}^{t 1} \mid=0 \\
& \sum_{t}\left(D_{0} \psi_{1}^{t}\right)^{3} Y_{0}^{t 1}\left|=0 ; \quad \sum_{t}\left(D_{0} \psi_{1}^{t}\right)^{4} Y_{0}^{t 1}\right|=|\eta|^{4} I^{5}
\end{aligned}
$$

d'où les $Y_{0}^{t 1} \mid$, puis les $Y_{0}^{t 1}$ et tous les $Y_{t}^{k}$.

1.5. Cas $(\mathbf{L G})_{2}$. La pente du 1er segment de $N$ est $\frac{1}{2}$. On distingue des sous cas

1) $b_{12}^{5} \neq 0$. Le 1er segment contient les points $0,(2,1),(4,2)$. Le fait que le 1er segment contient 3 points conduit, comme il est usuel lors de l'étude des diagrammes de Newton à distinguer des cas selon la nullité ou non nullité de discriminants.

1a) $\left(b_{04}^{5}\right)^{2}+4 b_{12}^{5}|\eta| \neq 0$. On cherche $f$ sous la forme

$$
\begin{aligned}
f & =\sum_{1 \leq t \leq 4} f_{1}^{t}+f_{2} ; \\
f_{1}^{t} & =e^{\psi^{t}}\left(Y_{0}^{t}+Y_{k}^{t}+\cdots\right) ;
\end{aligned}
$$

$D_{0} \psi^{t}$ est l'une des quatre racines distinctes non nulles de l'équation:

$$
\left(D_{0} \psi\right)^{4}-b_{04}^{5}|\eta|\left(D_{0} \psi\right)^{2}-b_{12}^{5}|\eta|^{3}=0 ; \quad \psi^{t} \mid=0 ;
$$

chaque $\psi^{t}$ est d'ordre $\frac{1}{2} ; Y_{k}^{t}$ est d'ordre: $\frac{4-k}{2}$;

$$
f_{2}=Z_{0}+\cdots+Z_{k}+\cdots
$$

$Z_{k}$ est d'ordre: $\frac{4-k}{2}$. 
1b) $\left(b_{04}^{5}\right)^{2}+4 b_{12}^{5}|\eta|=0 ; C \equiv \frac{b_{04}^{5} D_{0} b_{04}^{5}+b_{13}^{5} b_{04}^{5}|\eta|+2 b_{21}^{5}|\eta|^{2}}{2|\eta|^{2}} \equiv 0$.

$$
\begin{aligned}
f & =\sum_{1 \leq t \leq 2} f_{1}^{t}+f_{2} \\
f_{1}^{t} & =e^{\psi^{t}}\left(Y_{0}^{t}+\cdots+Y_{k}^{t}+\cdots\right)
\end{aligned}
$$

$D_{0} \psi^{t}$ est l'une des 2 racines distinctes non nulles de

$$
2\left(D_{0} \psi\right)^{2}-b_{04}^{5}|\eta|=0 ; \quad \psi^{t} \mid=0
$$

$\psi^{t}$ est d'ordre $\frac{1}{2} ; Y_{k}^{t}$ est d'ordre: $\frac{5-k}{2}$.

$$
f_{2}=Z_{0}+\cdots+Z_{k}+\cdots
$$

$Z_{k}$ est d'ordre: $\frac{4-k}{2}$.

1c) $\left(b_{04}^{5}\right)^{2}+4 b_{12}^{5}|\eta|=0 ; C \neq 0$.

$$
\begin{gathered}
f=\sum_{\substack{1 \leq t \leq 2 \\
1 \leq t^{\prime} \leq 2}} f_{1}^{t t^{\prime}}+f_{2} \\
f_{1}^{t t^{\prime}}=e^{\psi_{1}^{t}+\psi_{2}^{t t^{\prime}}}\left(Y_{0}^{t t^{\prime}}+\cdots+Y_{k}^{t t^{\prime}}+\cdots\right) ; \\
\left(D_{0} \psi_{1}^{t}\right)^{2}=\frac{b_{04}^{5}}{2}|\eta| \neq 0 ; \quad \psi_{1}^{t} \mid=0 ;
\end{gathered}
$$

$\psi_{1}^{t}$ est d'ordre $\frac{1}{2}$

$$
\left(D_{0} \psi_{2}^{t t^{\prime}}\right)^{2}=-\frac{C D_{0} \psi_{1}^{t}|\eta|^{2}}{b_{04}^{5}} \neq 0 ; \quad \psi_{2}^{t t^{\prime}} \mid=0
$$

les 4 phases $\psi_{2}^{t t^{\prime}}$ sont d'ordre: $\frac{1}{4} ; Y_{k}^{t t^{\prime}}$ est d'ordre: $\frac{9-k}{4}$.

$$
f_{2}=Z_{0}+\cdots+Z_{k}+\cdots
$$

$Z_{k}$ est d'ordre: $\frac{8-k}{4}$.

2) $b_{12}^{5}=0, b_{04}^{5} \neq 0$. On supprime le point $(4,2)$. Le 1er segment de $N$ contient les deux points 0 et $(2,1)$.

2a) $\quad b_{21}^{5} \neq 0$. Le 2ème segment du diagramme est défini par $(2,1)$ et $(5,2)$ de pente $\frac{1}{3}$. 
On cherche $f$ sous la forme:

$$
\begin{gathered}
f=\sum_{1 \leq t \leq 2} f_{1}^{t}+\sum_{1 \leq t^{\prime} \leq 3} f_{2}^{t^{\prime}} ; \\
f_{1}^{t}=e^{\psi^{t}}\left(Y_{0}^{t}+\cdots+Y_{k}^{t}+\cdots\right) \\
\left(D_{0} \psi^{t}\right)^{2}=b_{04}^{5}|\eta| \neq 0 ; \quad \psi^{t} \mid=0 ;
\end{gathered}
$$

$\psi^{t}$ est d'ordre $\frac{1}{2} ; Y_{k}^{t}$ est d'ordre: $\frac{12-k}{6}$;

$$
\begin{gathered}
f_{2}^{t^{\prime}}=e^{\psi^{t^{\prime}}}\left(Z_{0}^{t^{\prime}}+\cdots+Z_{k}^{t^{\prime}} \cdots\right) \\
b_{04}^{5}\left(D_{0} \chi^{t^{\prime}}\right)^{3}=b_{21}^{5}|\eta|^{3} ; \quad \chi^{t^{\prime}} \mid=0
\end{gathered}
$$

$\chi^{t^{\prime}}$ est d'ordre $\frac{1}{3} ; Z_{k}^{t^{\prime}}$ est d'ordre: $\frac{14-k}{6}$.

2b) $\quad b_{21}^{5} \equiv 0$. On supprime le point $(5,2)$. Le 2ème segment du diagramme contient les points $(2,1),(3,1),(4,1),(5,1)$ et est horizontal

$$
\begin{aligned}
& f=\sum_{1 \leq t \leq 2} f_{1}^{t}+f_{2} \\
& f_{1}^{t}=e^{\psi^{t}}\left(Y_{0}^{t}+\cdots+Y_{k}^{t}+\cdots\right) \\
& \left(D_{0} \psi^{t}\right)^{2}=b_{04}^{5}|\eta| \neq 0 ; \quad \psi^{t} \mid=0
\end{aligned}
$$

$\psi^{t}$ est d'ordre $\frac{1}{2} ; Y_{k}^{t}$ est d'ordre: $\frac{4-k}{2}$;

$$
f_{2}=Z_{0}+\cdots+Z_{k}+\cdots
$$

$Z_{k}$ est d'ordre: $\frac{6-k}{2}$.

On étudie ces différents cas

1a) Le système en $Y_{k}^{t}$ s'écrit:

(0) $\quad \mathrm{a} Y_{0}=0 ; \quad$ (1) $|\eta| \mathrm{a} Y_{1}+D_{0} \psi Y_{0}=0$;

(2) $|\eta| \mathrm{a} Y_{2}+\cdots+\mathrm{a} D_{*} \psi Y_{1}+D_{0} Y_{0}+b_{0} Y_{0}=0$;

(3) $|\eta| \mathrm{a} Y_{3}+\cdots+\left(D b_{0}\right)_{*}(\psi) Y_{0}+\mathrm{a} W(\psi ; 0) Y_{1}=0$;

(4) $|\eta| \mathrm{a} Y_{4}+\cdots+b_{1} Y_{0}+\left(D b_{0}\right)_{*}\left(Y_{0}\right)+\sum \mathrm{a} W_{j}\left(\psi ;-\frac{1}{2}\right) D_{j} Y_{1}$

$$
+\mathrm{a} W\left(\psi ;-\frac{1}{2}\right) Y_{1}+W(\psi ;-1) Y_{0}=0 ;
$$

le dernier $W$ est une somme de termes, qui commencent par des dérivées secondes de $b_{0}$ en $\xi$. 
(5) $|\eta| \mathrm{a} Y_{5}+\cdots+\left(D b_{1}\right)_{*}(\psi) Y_{0}+\sum \mathrm{a} W_{j k}(\psi ;-1) D_{j k} Y_{1}$

$$
+\sum \mathrm{a} W_{j}(\psi ;-1) D_{j} Y_{1}+\sum W_{j}\left(\psi ;-\frac{3}{2}\right) D_{j} Y_{0}+W\left(\psi ;-\frac{3}{2}\right) Y_{0}=0
$$

avec les derniers termes en $W$ commençant par des dérivés de $b_{0}$;

(6) $|\eta| \mathrm{a} Y_{6}+\cdots+b_{2} Y_{0}+\left(D b_{1}\right)_{*}\left(Y_{0}\right)+\sum_{j k} W_{j k}(\psi ;-2) D_{j k} Y_{0}$

$$
+\sum \mathrm{a} W_{j k}\left(\psi ;-\frac{3}{2}\right) D_{j k} Y_{1}=0
$$

avec un terme en $W_{j k}$ commençant par des dérivées de $b_{0}$;

(7) $\quad|\eta| \mathrm{a} Y_{8}+\cdots+\left(D b_{2}\right)_{*}(\psi) Y_{0}$

$+($ des termes qu'il ne sera pas nécessaire de préciser $)=0$.

On en déduit:

(0) $Y_{0}^{2}=Y_{0}^{3}=Y_{0}^{4}=Y_{0}^{5}=0$;

(1) $|\eta| Y_{1}^{2}+D_{0} \psi Y_{0}^{1}=0, \quad Y_{1}^{3}=Y_{1}^{4}=Y_{1}^{5}=0$;

(2) $|\eta| Y_{2}^{2}+\cdots+D_{0} Y_{0}^{1}-\frac{D_{0} \psi D_{*} \psi}{|\eta|} Y_{0}^{1}=0$,

$|\eta| Y_{2}^{3}-\frac{\left(D_{0} \psi\right)^{2}}{|\eta|} Y_{0}^{1}=0, \quad Y_{2}^{4}=Y_{2}^{5}=0$,

(3) $|\eta| Y_{3}^{2}+\cdots-\frac{D_{*} \psi}{|\eta|} D_{*} Y_{0}^{1}-\frac{D_{0} \psi}{|\eta|} D_{*} Y_{0}^{1}+W\left(\psi_{1} ;-\frac{1}{2}\right) Y_{0}^{1}=0$,

$|\eta| Y_{3}^{3}+\cdots-\frac{2 D_{0} \psi}{|\eta|} D_{0} Y_{0}^{1}+\left[\frac{2\left(D_{0} \psi\right)^{2} D_{*} \psi}{|\eta|^{2}}-\frac{D_{0}^{2} \psi}{|\eta|}\right] Y_{0}^{1}=0$,

$|\eta| Y_{3}^{4}+\frac{\left(D_{0} \psi\right)^{3}}{|\eta|^{2}} Y_{0}^{1}=0, \quad Y_{3}^{5}=0$

(4) $|\eta| Y_{4}^{3}+\cdots-\frac{D_{0}^{2} Y_{0}^{1}}{|\eta|}+W(\psi ;-1) D_{0} Y_{0}^{1}+\frac{2\left(D_{0} \psi\right)^{2}}{|\eta|^{2}} D_{*} Y_{0}^{1}+W(\psi ;-1) Y_{0}^{1}=0$

$|\eta| Y_{4}^{4}+\cdots+\frac{3\left(D_{0} \psi_{1}\right)^{2}}{|\eta|^{2}} D_{0} Y_{0}^{1}+\left[\frac{-3\left(D_{0} \psi\right)^{3} D_{*} \psi}{|\eta|^{3}}+\frac{3 D_{0} \psi D_{0}^{2} \psi}{|\eta|^{2}}\right] Y_{0}^{1}=0$

$|\eta| Y_{4}^{5}-\frac{\left(D_{0} \psi_{1}\right)^{4}}{|\eta|^{3}} Y_{0}^{1}=0$

(5) On obtient à la $5^{\text {ème }}$ ligne

$$
\left[\frac{\left(D_{0} \psi\right)^{5}}{|\eta|^{4}}-\frac{b_{04}^{5}\left(D_{0} \psi\right)^{3}}{|\eta|^{3}}-\frac{b_{12}^{5} D_{0} \psi}{|\eta|}\right] Y_{0}^{1}=0
$$


qui est réalisée sans restriction sur $Y_{0}^{1}$, par le choix de $D_{0} \psi$; on a aussi:

$$
\begin{aligned}
|\eta| Y_{5}^{4}+\cdots+ & \frac{D_{0} \psi}{|\eta|^{2}} D_{0}^{2} Y_{0}^{1}+W\left(\psi ;-\frac{3}{2}\right) D_{0} Y_{0}^{1} \\
& -\frac{3\left(D_{0} \psi\right)^{3}}{|\eta|^{3}} D_{*} Y_{0}^{1}+W\left(\psi ;-\frac{3}{2}\right) Y_{0}^{1}=0 \\
|\eta| Y_{5}^{5}+\cdots- & \frac{4\left(D_{0} \psi\right)^{3}}{|\eta|^{3}} D_{0} Y_{0}^{1}+\left[\frac{4\left(D_{0} \psi\right)^{4} D_{*} \psi}{|\eta|^{4}}-\frac{6\left(D_{0} \psi\right)^{2} D_{0}^{2} \psi}{|\eta|^{3}}\right] Y_{0}^{1}=0
\end{aligned}
$$

(6) On obtient à la $5^{\text {ème }}$ ligne

$$
\begin{aligned}
& {\left[\frac{5\left(D_{0} \psi\right)^{4}}{|\eta|^{4}}-\frac{3 b_{04}^{5}}{|\eta|^{3}}\left(D_{0} \psi\right)^{2}-\frac{b_{12}^{5}}{|\eta|}\right] D_{0} Y_{0}^{1}+\left[-\frac{4\left(D_{0} \psi\right)^{5} D_{*} \psi}{|\eta|^{5}}\right.} \\
& \quad+10 \frac{\left(D_{0} \psi\right)^{3} D_{0}^{2} \psi}{|\eta|^{4}}+3 b_{04}^{5} \frac{\left(D_{0} \psi\right)^{3} D_{*} \psi}{|\eta|^{4}}-\frac{3 b_{04}^{5} D_{0} \psi D_{0}^{2} \psi}{|\eta|^{3}}+\frac{b_{12}^{5}}{|\eta|^{2}} D_{*} \psi D_{0} \psi \\
& \left.\quad+b_{13}^{5} \frac{\left(D_{0} \psi\right)^{2}}{|\eta|^{2}}-\left(D b_{04}^{5}\right)_{*}(\psi) \frac{\left(D_{0} \psi\right)^{3}}{|\eta|^{3}}-\left(D b_{12}^{5}\right)_{*}(\psi) \frac{D_{0} \psi}{|\eta|}+b_{21}^{5}\right] Y_{0}=0
\end{aligned}
$$

le coefficient de $D_{0} Y_{0}^{1}$ s'écrit aussi:

$$
2\left[2\left(D_{0} \psi\right)^{2}-b_{04}^{5}|\eta|\right] \frac{\left(D_{0} \psi\right)^{2}}{|\eta|^{4}}
$$

et comme le discriminant: $\left[\left(b_{04}^{5}\right)^{2}+4 b_{12}^{5}|\eta|\right]|\eta|^{2}$ n'est pas nul, il n'est pas nul. Il reste à déterminer $\left.Y_{0}^{1}\right|_{x_{0}=\underline{x}_{0}}$.

Le coefficient de $Y_{0}^{1}$ sera utile dans le sous cas suivant. On a aussi:

$$
\begin{aligned}
|\eta| Y_{6}^{5}+\cdots-4 \frac{\left(D_{0} \psi\right)^{2}}{|\eta|^{3}} & D_{0}^{2} Y_{0}^{1}+W(\psi ;-2) D_{0} Y_{0}^{1} \\
& +4 \frac{\left(D_{0} \psi\right)^{4}}{|\eta|^{4}} D_{*} Y_{0}^{1}+W(\psi ;-2) Y_{0}^{1}=0
\end{aligned}
$$

Pour les $Z$, on a:
(0) (1) $\mathrm{a} Z_{0}=\mathrm{a} Z_{1}=0$
(2) $|\eta| \mathrm{a} Z_{2}+D_{0} Z_{0}+b_{0} Z_{0}=0$
(3) $|\eta| \mathrm{a} Z_{3}+\cdots=0$
(4) $|\eta| \mathrm{a} Z_{4}+\cdots+b_{1} Z_{0}+\mathrm{a} D_{*} Z_{2}+\left(D b_{0}\right)_{*}\left(Z_{0}\right)=0$
(5) $|\eta| \mathrm{a} Z_{5}+\cdots=0$
(6) $|\eta| \mathrm{a} Z_{6}+\cdots+b_{2} Z_{0}+\left(D b_{1}\right)_{*}\left(Z_{0}\right)+\sum_{\left|\alpha^{\prime}\right|=2} W_{\alpha^{\prime}}(-2) D^{\alpha^{\prime}} Z_{0}=0$ 
La $5^{\text {ème }}$ ligne des termes en $W$ est nulle. On a donc:

(0) (1) $Z_{0}^{2}=Z_{0}^{3}=Z_{0}^{4}=Z_{0}^{5}=Z_{1}^{2}=Z_{1}^{3}=Z_{1}^{4}=Z_{1}^{5}=0$;

(2) $|\eta| Z_{2}^{2}+D_{0} Z_{0}^{1}=0, \quad Z_{2}^{3}=Z_{2}^{4}=Z_{2}^{5}=0$;

(3) $Z_{3}^{3}=Z_{3}^{4}=Z_{3}^{5}=0$;

(4) $|\eta| Z_{4}^{3}-\frac{D_{0}^{2} Z_{0}^{1}}{|\eta|}=0, \quad Z_{4}^{4}=Z_{4}^{5}=0$;

(5) $Z_{5}^{4}=Z_{5}^{5}=0$;

à l'étape (6), la 5 ème ligne donne:

$$
-b_{12}^{5} D_{0} Z_{0}^{1}+|\eta| b_{21}^{5} Z_{0}^{1}=0 .
$$

Il reste à déterminer $\left.Z_{0}^{1}\right|_{x_{0}=\underline{x}_{0}}$.

On imposera les conditions initiales:

$$
\begin{aligned}
\sum_{t} Y_{0}^{t}\left|+Z_{0}\right| & =0=\sum_{t} Y_{1}^{t}\left|+Z_{1}\right|=\sum_{t} Y_{2}^{t}\left|+Z_{2}\right|=\sum_{t} Y_{3}^{t}\left|+Z_{3}\right| \\
\sum_{t} Y_{4}^{t} \mid+Z_{4} & =I, \quad \cdots
\end{aligned}
$$

on obtient:

$$
\begin{aligned}
& \sum_{t} Y_{0}^{t 1}\left|+Z_{0}^{1}\right|=0=\sum_{t} D_{0} \psi^{t} Y_{0}^{t 1}=0 \\
& \sum_{t}\left(D_{0} \psi^{t}\right)^{2} Y_{0}^{t 1}\left|=0 ; \quad \sum_{t}\left(D_{0} \psi\right)^{3} Y_{0}^{t 1}\right|=0 ;\left.\quad \sum_{t}\left(D_{0} \psi^{t}\right)^{4} Y_{0}^{t 1}|=| \eta\right|^{4} I^{5}
\end{aligned}
$$

la dernière équation s'écrit aussi, compte tenu de ce qui précède

$$
b_{12}^{5} \sum_{t} Y_{0}^{t 1}|=| \eta \mid I^{5}
$$

comme précédemment, on a un déterminant de Vandermonde non nul, puisque les $D_{0} \psi^{t}$ sont distincts et on détermine les quatre $Y_{0}^{t 1} \mid$ d'où les $Y_{0}^{t 1}$; on a aussi $Z_{0}^{1} \mid$, puis $Z_{0}^{1}$ et tous les coefficients.

1b) Les calculs des $Y$ sont semblables jusqu'à l'étape (6) à ceux du cas 1a); à l'étape (6), dans les conditions du $1 \mathrm{~b}$ ), le coefficient de $D_{0} Y_{0}^{1}$ est nul; le coefficient de $Y_{0}^{1}$ est égal à $C$, compte tenu de la nullité du discriminant, et par la suite dans le 1b) il est nul.

A l'étape (7), on obtient à la $5^{\text {ème }}$ ligne:

$$
\frac{8\left(D_{0} \psi\right)^{3}}{|\eta|^{4}}-\frac{b_{04}^{5} D_{0} \psi}{|\eta|^{3}} D_{0}^{2} Y_{0}^{1}+W\left(\psi ;-\frac{5}{2}\right) D_{0} Y_{0}^{1}+W^{\prime}\left(\psi ;-\frac{5}{2}\right) Y_{0}^{1}=0
$$


le coefficient de $D_{0}^{2} Y_{0}^{1}$ est non nul, il reste à déterminer $D_{0} Y_{0}^{1} \mid$ et $Y_{0}^{1} \mid$.

$\operatorname{Par} Z$, comme précédemment, il reste à déterminer $Z_{0}^{1} \mid$. On impose les conditions:

$$
\begin{array}{llll}
\sum_{t} Y_{0}^{t} \mid=0 ; & \sum_{t} Y_{1}^{t}\left|+Z_{0}\right|=0 ; & \sum_{t} Y_{2}^{t}\left|+Z_{1}\right|=0 ; & \sum_{t} Y_{3}^{t}\left|+Z_{2}^{t}\right|=0 ; \\
\sum_{t} Y_{4}^{t}\left|+Z_{3}\right|=0 ; & \sum_{t} Y_{5}^{t}+Z_{4} \mid=I ; & \sum_{t} Y_{6}^{t}+Z_{5} \mid=0 .
\end{array}
$$

On a:

$$
\begin{aligned}
& \sum_{t} Y_{0}^{t 1}\left|=0 ; \quad \sum_{t} Y_{1}^{t 1}\right|+Z_{0}^{1}\left|=0 ; \quad \sum_{t} D_{0} \psi^{t} Y_{0}^{t 1}\right|=0 \\
& \sum_{t} D_{0} \psi^{t} Y_{1}^{t 1}\left|+\sum_{t} D_{0} Y_{0}^{t 1}\right|=0 ; \quad b_{04}^{5}|\eta| \sum_{t} Y_{1}^{t}\left|+4 \sum D_{0} \psi^{t} D_{0} Y_{0}^{t 1}\right|=0 \\
& \sum_{t} D_{0} \psi^{t} Y_{1}^{t 1}\left|+3 \sum_{t} D_{0} \psi^{t} Y_{0}^{t 1}\right|=0 ; \\
& |\eta|\left(b_{04}^{5}\right)^{2} \sum_{t} Y_{1}^{t 1}\left|+8 b_{04}^{5} \sum D_{0} \psi^{t} D_{0} Y_{0}^{t 1}\right|=4|\eta|^{3} I^{5} ; \quad \ldots
\end{aligned}
$$

on en déduit: $Y_{0}^{t 1} \mid=0$, pour chaque $t ; \sum_{t} D_{0} \psi^{t} D_{0} Y_{0}^{t 1}\left|=\frac{|\eta|^{3}}{b_{04}^{5} \mid} I^{5} ; \sum_{t} D_{0} Y_{0}^{t 1}\right|=0$, d'où les restrictions des $D_{0} Y_{0}^{t 1}$ et par suite tous les $Y_{0}^{t}$; on a aussi: $\sum_{t} Y_{1}^{t 1} \mid$ et par suite $Z_{0}^{1} \mid$ et les $Z_{0}$ et tous les coefficients.

1c) On a:

(0) $\quad \mathrm{a} Y_{0}=0 ; \quad(1) \quad \mathrm{a} Y_{1}=0$;

(2) $|\eta| \mathrm{a} Y_{2}+D_{0} \psi_{1} Y_{0}=0 ; \quad$ (3) $\quad|\eta| \mathrm{a} Y_{3}+\cdots+D_{0} \psi_{2} Y_{0}=0$;

(4) $|\eta| \mathrm{a} Y_{4}+\cdots+D_{0} Y_{0}+b_{0} Y_{0}=0 ; \quad$ (5) $\quad|\eta| \mathrm{a} Y_{5}+\cdots=0$

(6) $|\eta| \mathrm{a} Y_{6}+\cdots+W\left(\psi_{1} ; 0\right) \mathrm{a} Y_{2}+\left(D b_{0}\right)_{*}\left(\psi_{1}\right) Y_{0}=0$;

(7) $|\eta| \mathrm{a} Y_{7}+\cdots+W\left(\psi_{1}, \psi_{2} ;-\frac{1}{4}\right) \mathrm{a} Y_{2}+\left(D b_{0}\right)_{*}\left(\psi_{2}\right) Y_{0}=0$;

(8) $|\eta| \mathrm{a} Y_{8}+\cdots+\sum_{j} W_{j}\left(\psi_{1} ;-\frac{1}{2}\right) \mathrm{a} \partial_{j} Y_{2}+W^{\prime}\left(\psi_{2} ; \frac{1}{2}\right) \mathrm{a} Y_{2}$

$$
+b_{1} Y_{0}+\left(D b_{0}\right)_{*}\left(Y_{0}\right)+W\left(\psi_{1} ;-1\right) Y_{0}=0 \text {. }
$$

Le dernier terme commence par une dérivée de $b_{0}$;

(9) $|\eta| \mathrm{a} Y_{9}+\cdots+\sum_{j} W_{j}\left(\psi_{2} ;-\frac{3}{4}\right) \mathrm{a} D_{j} Y_{2}$

$$
+W\left(\psi_{1}, \psi_{2} ;-\frac{3}{4}\right) \mathrm{a} Y_{2}+W\left(\psi_{1}, \psi_{2} ;-\frac{5}{4}\right) Y_{0}=0 .
$$


Le dernier terme commence par une dérivée de $b_{0}$;

$$
\begin{aligned}
& \text { (10) }|\eta| \mathrm{a} Y_{10}+\cdots+\left(D b_{1}\right)_{*}\left(\psi_{1}\right) Y_{0}+\cdots=0 \\
& \text { (11) }|\eta| \mathrm{a} Y_{11}+\cdots+\left(D b_{1}\right)_{*}\left(\psi_{2}\right) Y_{0}+\cdots=0 \\
& \text { (12) }|\eta| \mathrm{a} Y_{12}+\cdots+b_{2} Y_{0}+\left(D b_{1}\right)_{*}\left(Y_{0}\right)+\cdots=0 \\
& \text { (13) }|\eta| \mathrm{a} Y_{13}+\cdots=0
\end{aligned}
$$

dans les équations (10), (11), (12), (13), nous n'avons écrit que quelques termes pour guider le lecteur.

On en déduit, en résumant:

(0) $Y_{0}^{2}=Y_{0}^{3}=Y_{0}^{4}=Y_{0}^{5}=0$;

(1) $Y_{1}^{2}=Y_{1}^{3}=Y_{1}^{4}=Y_{1}^{5}=0$;

(2) $|\eta| Y_{2}^{2}+D_{0} \psi_{1} Y_{0}^{1}=0 ; \quad Y_{2}^{3}=Y_{2}^{4}=Y_{2}^{5}$;

(3) $|\eta| Y_{3}^{2}+\cdots+D_{0} \psi_{2} Y_{0}^{1}=0, \quad Y_{3}^{3}=Y_{3}^{4}=Y_{3}^{5}=0$;

(4) $|\eta| Y_{4}^{2}+\cdots+D_{0} Y_{0}^{1}-\frac{D_{*} \psi_{1} D_{0} \psi_{1}}{|\eta|} Y_{0}^{1}=0$;

$$
|\eta| Y_{4}^{3}-\frac{\left(D_{0} \psi_{1}\right)^{2}}{|\eta|}=0, \quad Y_{4}^{4}=Y_{4}^{5}=0
$$

(5) $|\eta| Y_{5}^{3}+\cdots-\frac{2 D_{0} \psi_{1} D_{0} \psi_{2}}{|\eta|}=0, \quad Y_{5}^{4}=Y_{5}^{5}=0$;

(6) $|\eta| Y_{6}^{4}+\frac{\left(D_{0} \psi_{1}\right)^{3}}{|\eta|^{2}} Y_{0}^{1}=0$,

$$
|\eta| Y_{6}^{3}-\frac{2 D_{0} \psi_{1}}{|\eta|} D_{0} Y_{0}^{1}+\left[-\frac{\left(D_{0} \psi_{2}\right)^{2}}{|\eta|}+W\left(\psi_{1} ;-\frac{1}{2}\right)\right] Y_{0}^{1}=0 ; \quad Y_{6}^{5}=0
$$

(7) $|\eta| Y_{7}^{4}+\cdots+\frac{3\left(D_{0} \varphi_{1}\right)^{2} D_{0} \psi_{2}}{|\eta|^{2}}=0$,

$|\eta| Y_{7}^{3}+\cdots-\frac{2 D_{0} \psi_{2}}{|\eta|} D_{0} Y_{0}^{1}=0, \quad Y_{7}^{5}=0 ;$

(8) $|\eta| Y_{8}^{5}-f\left(\left(D_{0} \psi_{1}\right)^{4} ;|\eta|^{3}\right) Y_{0}^{1}=0$,

$$
|\eta| Y_{8}^{4}+\cdots+3 \frac{\left(D_{0} \psi_{1}\right)^{2}}{|\eta|^{2}} D_{0} Y_{0}^{1}+\frac{3 D_{0} \psi_{1}\left(D_{0} \psi_{2}\right)^{2}}{|\eta|^{2}}+W\left(\psi_{1} ;-1\right) Y_{0}^{1}=0
$$

(9) $|\eta| Y_{9}^{5}+\cdots-\frac{4\left(D_{0} \psi_{1}\right)^{3} D_{0} \psi_{2}}{|\eta|^{2}} Y_{0}^{1}=0$

$$
|\eta| Y_{9}^{4}+\cdots-\frac{6 D_{0} \psi_{1} D_{0} \psi_{2}}{|\eta|^{2}} D_{0} Y_{0}^{1}+W\left(\psi_{1}, \psi_{2} ;-\frac{5}{4}\right) Y_{0}^{1}=0
$$

(10) La $5^{\text {ème }}$ ligne est réalisée sans condition sur $Y_{0}^{1}$, du fait que:

$$
\left(D_{0} \psi_{1}\right)^{4}-b_{04}^{5}|\eta|\left(D_{0} \psi_{1}\right)^{2}-b_{12}^{5}|\eta|^{3}=0 ;
$$


on a aussi:

(11) La $5^{\text {ème }}$ ligne est réalisée sans condition sur $Y_{0}^{1}$ du fait que $D_{0} \psi_{1}$ est racine double.

$$
|\eta| Y_{11}^{5}+\cdots-12 \frac{\left(D_{0} \psi_{1}\right)^{2} D_{0} \psi_{2}}{|\eta|^{3}} D_{0} Y_{0}^{1}+W\left(\psi_{1}, y_{2} ;-\frac{7}{4}\right) Y_{0}^{1}=0
$$

(12) La $5^{\text {ème }}$ ligne est réalisée sans condition sur $Y_{0}^{1}$ du fait du choix de $D_{0} \psi_{2}$.

(13) La $5^{\text {ème }}$ ligne donne:

$$
8\left(D_{0} \psi_{1}\right)^{3} D_{0} \psi_{2} D_{0} Y_{0}^{1}+W\left(\psi_{1}, \psi_{2} ; \frac{7}{4}\right) Y_{0}^{1}=0
$$

il suffit de déterminer $Y_{0}^{1} \mid$.

Pour $Z$, on a:

$$
\begin{aligned}
& \mathrm{a} Z_{0}=\mathrm{a} Z_{1}=\mathrm{a} Z_{2}=\mathrm{a} Z_{3}=0 ; \quad|\eta| \mathrm{a} Z_{4}+D_{0} Z_{0}+b_{0} Z_{0}=0 ; \quad \ldots ; \\
& |\eta| \mathrm{a} Z_{8}+D_{0} Z_{4}+\mathrm{a} D_{*} Z_{4}+b_{0} Z_{4}+b_{1} Z_{0}+\left(D b_{0}\right)_{*} Z_{4}=0 ; \\
& |\eta| \mathrm{a} Z_{12}+\cdots+b_{2} Z_{0}+\left(D b_{1}\right)_{*}\left(Z_{0}\right)+\sum_{|\alpha|=2} \frac{\partial_{\alpha^{\prime}} b_{0}}{a^{\prime} !} D^{\alpha^{\prime}} Z_{0}=0 ; \quad \ldots
\end{aligned}
$$

On en déduit:

$$
\begin{array}{lll}
Z_{0}^{2}=Z_{0}^{3}=Z_{0}^{4}=Z_{0}^{5}=\cdots=Z_{3}^{5}=0 ; & & |\eta| Z_{4}^{2}+D_{0} Z_{0}^{1}=0 ; \\
Z_{4}^{3}=Z_{4}^{4}=Z_{4}^{5}=0 ; & Z_{5}^{3}=Z_{5}^{4}=Z_{5}^{5}=0 ; & Z_{6}^{3}=Z_{6}^{4}=Z_{6}^{5}=0 ; \\
Z_{7}^{3}=Z_{7}^{4}=Z_{7}^{5}=0 ; & |\eta| Z_{8}^{3}-\frac{1}{|\eta|} D_{0}^{2} Z_{0}^{1}=0 ; & Z_{8}^{4}=Z_{8}^{5}=0 ; \\
Z_{9}^{4}=Z_{9}^{5}=0 ; & Z_{10}^{4}=Z_{10}^{5}=0 ; & -b_{12}^{5} D_{0} Z_{0}^{1}+|\eta| b_{21}^{5} Z_{0}^{1}=0 ; \ldots
\end{array}
$$

cette dernière équation détermine $Z_{0}^{1}$ si $\left.Z_{0}^{1}\right|_{x_{0}=\underline{x}_{0}}$ est connu.

On impose les conditions initiales.

$$
\begin{aligned}
& \sum_{t t^{\prime}} Y_{0}^{t t^{\prime}}\left|=0 ; \quad \sum_{t t^{\prime}} Y_{1}^{t t^{\prime}}\right|+Z_{0}=0 ; \quad \sum_{t t^{\prime}} Y_{2}^{t t^{\prime}}\left|+Z_{1}\right|=0 ; \quad \sum_{t t^{\prime}} Y_{3}^{t t^{\prime}}\left|+Z_{2}\right|=0 \\
& \sum_{t t^{\prime}} Y_{4}^{t t^{\prime}}+Z_{3}\left|=0 ; \quad \sum_{t t^{\prime}} Y_{5}^{t t^{\prime}}+Z_{4}\right|=0 ; \quad \sum_{t t^{\prime}} Y_{6}^{t t^{\prime}}+Z_{5}\left|=0 ; \quad \sum_{t t^{\prime}} Y_{7}^{t t^{\prime}}+Z_{6}\right|=0 \\
& \sum_{t t^{\prime}} Y_{8}^{t t^{\prime}}+Z_{7}\left|=0 ; \quad \sum_{t t^{\prime}} Y_{9}^{t t^{\prime}}+Z_{8}\right|=I ; \ldots
\end{aligned}
$$

On en déduit:
(0) $\sum_{t t^{\prime}} Y_{0}^{t t^{\prime} 1}=0$
(1) $\sum_{t t^{\prime}} Y_{1}^{t t^{\prime} 1}\left|+Z_{0}^{1}\right|=0$
(2) $\quad \sum_{t t^{\prime}} D_{0} \psi_{1}^{t} Y_{0}^{t t^{\prime} 1} \mid=0$ 
(3) $\sum_{t t^{\prime}} D_{0} \psi_{1}^{t} Y_{1}^{t t^{\prime} 1}\left|+\sum_{t t^{\prime}} D_{0} \psi_{2}^{t^{\prime}} Y_{0}^{t t^{\prime} 1}\right|=0$;

(5) $|\eta| \frac{b_{04}^{5}}{2} \sum_{t t^{\prime}} Y_{1}^{t t^{\prime} 1}\left|+2 \sum_{t t^{\prime}} D_{0} \psi_{1}^{t} D_{0} \psi_{2}^{t^{\prime}} Y_{0}^{t t^{\prime} 1}\right|=0$;

(7) $\sum_{t t^{\prime}} D_{0} \psi_{1}^{t} Y_{1}^{t t^{\prime} 1}\left|+3 \sum_{t t^{\prime}} D_{0} \psi_{2}^{t^{\prime}} Y_{0}^{t t^{\prime} 1}\right|=0$

(9) $|\eta| \frac{\left(b_{04}^{5}\right)^{2}}{4} \sum_{t t^{\prime}} Y_{1}^{t t^{\prime} 1}\left|+2 b_{04}^{5} \sum_{t t^{\prime}} D_{0} \psi_{1}^{t} D_{0} \psi_{2}^{t^{\prime}} Y_{0}^{t t^{\prime} 1}\right|=|\eta|^{3} I^{5}$

On en déduit:
(0) $\sum_{t t^{\prime}} Y_{0}^{t t^{\prime} 1} \mid=0$;
(2) $\sum_{t t^{\prime}} D_{0} \psi_{1}^{t} Y_{0}^{t t^{\prime} 1} \mid=0$
(3) (7) $\quad \sum_{t t^{\prime}} D_{0} \psi_{2}^{t t^{\prime}} Y_{0}^{t t^{\prime} 1} \mid=0$;
(5) (9) $\sum_{t t^{\prime}} D_{0} \psi_{1}^{t} D_{0} \psi_{2}^{t t^{\prime}} Y_{0}^{t t^{\prime} 1} \mid=\frac{|\eta|^{3} I^{5}}{b_{04}^{5}}$

on déduit de (0) et (2):

$$
Y_{0}^{111}\left|+Y_{0}^{121}\right|=0 ; \quad Y_{0}^{211}\left|+Y_{0}^{221}\right|=0 ;
$$

on déduit de (0) et $(3),(7)$ :

$$
D_{0} \psi_{2}^{11} Y_{0}^{111}\left|+D_{0} \psi_{2}^{21} Y_{0}^{211}\right|=0
$$

on en déduit alors de (5) (9) et $(*)$ :

$$
D_{0} \psi_{1}^{1} D_{0} \psi_{2}^{11} \psi_{0}^{111}\left|+D_{0} \psi_{1}^{2} D_{0} \psi_{2}^{21} Y_{0}^{211}\right|=\frac{|\eta|^{3} I^{5}}{2 b_{04}^{5} \mid}
$$

on obtient les valeurs de: $D_{0} \psi_{2}^{11} Y_{0}^{111} \mid$ et $D_{0} \psi_{2}^{21} Y_{0}^{211} \mid$ et par la suite tous les $Y_{0}^{t t^{\prime} 1} \mid$, donc tous les $Y_{0}^{t t^{\prime} 1}$ et les $Y_{k}^{t t^{\prime}}$; on a aussi $Z_{0}^{1} \mid$ et ensuite $Z_{0}^{1}$ et les $Z_{k}$.

2a) Les calculs ne présentent pas de particularité nouvelle; on a d'abord:
(0) $\mathrm{a} Y_{0}=0$;
(1) $\mathrm{a} Y_{1}=0$;
(2) $\mathrm{a} Y_{2}=0$
(3) $|\eta| \mathrm{a} Y_{3}+D_{0} \psi Y_{0}=0$
(4) $|\eta| \mathrm{a} Y_{4}+\cdots=0$;
(5) $|\eta| \mathrm{a} Y_{5}+\cdots=0$;
(6) $|\eta| \mathrm{a} Y_{6}+\cdots+D_{0} Y_{0}+b_{0} Y_{0}+\mathrm{a} D_{*} \psi Y_{3}=0$;
(7) $|\eta| \mathrm{a} Y_{7}+\cdots=0$;
(8) $|\eta| \mathrm{a} Y_{8}+\cdots=0$;
(9) $|\eta| \mathrm{a} Y_{9}+\cdots+\left(D b_{0}\right)_{*}(\psi) Y_{0}+W(\psi ; 0) \mathrm{a} Y_{3}=0$
(10) $|\eta| \mathrm{a} Y_{10}+\cdots=0$;
(11) $|\eta| \mathrm{a} Y_{11}+\cdots=0$. 
Nous n'indiquons ensuite que quelques termes pour guider le lecteur:

(12) $|\eta| \mathrm{a} Y_{12}+\cdots+b_{1} Y_{0}+\left(D b_{0}\right)_{*}\left(Y_{0}\right)+\cdots=0$

(13) $|\eta| \mathrm{a} Y_{13}+\cdots=0$;

(14) $|\eta| \mathrm{a} Y_{14}+\cdots=0$;

(15) $|\eta| \mathrm{a} Y_{15}+\cdots+\left(D b_{1}\right)_{*}(\psi) Y_{0}+\cdots=0$;

(16) $|\eta| \mathrm{a} Y_{16}+\cdots=0$

(17) $|\eta| \mathrm{a} Y_{17}+\cdots=0$;

(18) $|\eta| \mathrm{a} Y_{18}+\cdots+b_{2} Y_{0}+\left(D b_{1}\right)_{*}\left(Y_{0}\right)+\cdots=0$.

On a en résumé:

(0) $Y_{0}^{2}=Y_{0}^{3}=Y_{0}^{4}=Y_{0}^{5}=0$;

(3) $|\eta| Y_{3}^{2}+D_{0} \psi Y_{0}^{1}=0$

(6) $|\eta| Y_{6}^{2}+\cdots+D_{0} Y_{0}^{1}+W(\psi ; 0) Y_{0}^{1}=0$; $|\eta| Y_{6}^{3}-\frac{\left(D_{0} \psi\right)^{2}}{|\eta|} Y_{0}^{1}=0$;

(9) $|\eta| Y_{9}^{3}-\frac{2 D_{0} \psi}{|\eta|} D_{0} Y_{0}^{1}+W\left(\psi,-\frac{1}{2}\right) Y_{0}^{1}=0$; $|\eta| Y_{9}^{4}+\frac{\left(D_{0} \psi\right)^{3}}{|\eta|^{2}} Y_{0}^{1}=0$ $|\eta| Y_{12}^{4}+3 \frac{\left(D_{0} \psi\right)^{2}}{|\eta|^{2}} D_{0} Y_{0}^{1}+W(\psi ;-1) Y_{0}^{1}=0 ; \quad|\eta| Y_{12}^{5}-\frac{\left(D_{0} \psi\right)^{4}}{|\eta|^{3}} Y_{0}^{1}=0$

à la $15^{\text {ème }}$ étape, la $5^{\text {ème }}$ ligne est réalisée sans condition sur $Y_{0}^{1}$ du fait que: $\left(D_{0} \psi\right)^{2}=b_{04}^{5}|\eta| ;$ on a aussi

$$
|\eta| Y_{15}^{5}-4 \frac{\left(D_{0} \psi\right)^{3}}{|\eta|^{3}} D_{0} Y_{0}^{1}+W\left(\psi ;-\frac{3}{2}\right) Y_{0}^{1}=0 ;
$$

à la $18^{\text {ème }}$ étape, la $5^{\text {ème }}$ ligne donne après simplification:

$$
2 \frac{\left(D_{0} \psi\right)^{4}}{|\eta|^{4}} D_{0} Y_{0}^{1}+W\left(\psi_{2} ;-2\right) Y_{0}^{1}=0
$$

il reste à déterminer $\left.Y_{0}^{1}\right|_{x_{0}=\underline{x}_{0}}$.

Pour les $Z$, on obtient:

(0) (1) (2) (3) $\mathrm{a} Z_{0}=\mathrm{a} Z_{1}=\mathrm{a} Z_{2}=\mathrm{a} Z_{3}=0 ; \quad$ (4) $|\eta| \mathrm{a} Z_{4}+D_{0} \chi Z_{0}=0$;

(5) $|\eta| \mathrm{a} Z_{5}+D_{0} \chi Z_{1}=0 ; \quad$ (6) $|\eta| \mathrm{a} Z_{6}+D_{0} \chi Z_{2}+D_{0} Z_{0}+b_{0} Z_{0}=0$;

à l'étape (12) apparaît $b_{1} Z_{0}$ et à l'étape (18) $b_{2} Z_{0}$; on écrit (19) et (20); on a en résumé:

(0) $Z_{0}^{2}=Z_{0}^{3}=Z_{0}^{4}=Z_{0}^{5}=0$;

(4) $|\eta| Z_{4}^{2}+D_{0} \chi Z_{0}^{1}=0$;

(6) $|\eta| Z_{6}^{2}+\cdots+D_{0} Z_{0}^{1}+W(\chi ; 0) Z_{0}^{1}=0 ;|\eta| Z_{8}^{3}-\frac{\left(D_{0} \chi\right)^{2} Z_{0}^{1}}{|\eta|}=0$; 


$$
\begin{aligned}
& |\eta| Z_{10}^{3}+\cdots-2 \frac{D_{0} \chi}{|\eta|} D_{0} Z_{0}^{1}+W\left(\chi ;-\frac{2}{3}\right) Z_{0}^{1}=0 ; \quad|\eta| Z_{12}^{4}+\frac{\left(D_{0} \chi\right)^{3}}{|\eta|^{2}} Z_{0}^{1}=0 \\
& |\eta| Z_{14}^{4}+\cdots+3 \frac{\left(D_{0} \chi\right)^{2}}{|\eta|^{2}} D_{0} Z_{0}^{1}+W\left(\chi ;-\frac{4}{3}\right) Z_{0}^{1}=0 ; \quad|\eta| Z_{16}^{5}-\frac{\left(D_{0} \chi\right)^{4}}{|\eta|^{3}} Z_{0}^{1}=0
\end{aligned}
$$

à la $18^{\text {ème }}$ étape, on obtient:

$$
\left[-b_{04}^{5} \frac{\left(D_{0} \chi\right)^{3}}{|\eta|^{3}}+b_{21}^{5}\right] Z_{0}^{1}=0
$$

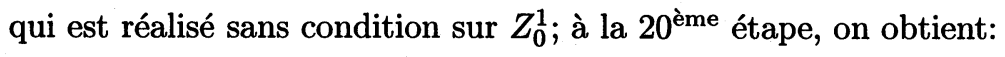

$$
-3 b_{04}^{5} \frac{\left(D_{0} \chi\right)^{2}}{|\eta|^{3}} D_{0} Z_{0}^{1}+W\left(\psi ;-\frac{7}{3}\right) Z_{0}^{1}=0
$$

il reste donc $\left.Z_{0}^{1}\right|_{x_{0}=\underline{x}_{0}}$ à déterminer.

Les conditions initiales imposées sont:

$$
\begin{gathered}
\sum_{t^{\prime}} Z_{0}^{t^{\prime}}\left|=0 ; \quad \sum_{t^{\prime}} Z_{1}^{t^{\prime}}\right|=0 ; \quad \sum_{t} Y_{0}^{t}\left|+\sum_{t^{\prime}} Z_{2}^{t^{\prime}}\right|=0 \\
\ldots ; \quad \sum_{t} Y_{12}^{t}\left|+\sum_{t^{\prime}} Z_{14}^{t^{\prime}}\right|=I ; \quad \ldots
\end{gathered}
$$

d'où:

$$
\begin{aligned}
& \sum_{t^{\prime}} Z_{0}^{t^{\prime} 1}\left|=0 ; \quad \sum_{t^{\prime}} D_{0} \chi^{t^{\prime}} Z_{0}^{t^{\prime} 1}\right|=0 ; \quad b_{04}^{5}|\eta| \sum_{t} Y_{0}^{t 1}\left|+\sum_{t^{\prime}}\left(D_{0} \chi^{t^{\prime}}\right)^{2} Z_{0}^{t^{\prime} 1}\right|=0 \\
& \sum_{t} D_{0} \psi^{t} Y_{0}^{t 1}\left|=0 ; \quad\left(b_{04}^{5}\right)^{2} \sum_{t} Y_{0}^{t 1}\right|=|\eta|^{2} I^{5}
\end{aligned}
$$

on obtient donc les $Y_{0}^{t 1}$ et les $Y_{0}^{t 1}$ et tous les $Y_{k}^{t}$, on a aussi alors les $Z_{0}^{t^{\prime} 1}$, puis les $Z_{0}^{t^{\prime} 1}$ et tous les $Z_{k}^{t^{\prime}}$.

2b) Pour les $Y$, il suffit de reprendre les calculs du cas 1a); à l'étape (5), le choix de $D_{0} \psi$ laisse $Y_{0}^{1}$ libre; à l'étape (6) on obtient:

$$
2 \frac{\left(D_{0} \psi\right)^{4}}{|\eta|^{4}} D_{0} Y_{0}^{1}+W(\psi ;-2) Y_{0}^{1}=0
$$

et il reste $\left.Y_{0}^{1}\right|_{x_{0}=\underline{x}_{0}}$ à déterminer. Pour les $Z$, de même, à l'étape (8), on a:

$$
b_{04}^{5} D_{0}^{3} Z_{0}^{1}+W(0) D_{0}^{2} Z_{0}^{1}+W^{\prime}(0) D_{0} Z_{0}^{1}+W^{\prime \prime}(0) Z_{0}^{1}=0
$$

il reste à déterminer $\left.Z_{0}^{1}\right|_{x_{0}=\underline{x}_{0}},\left.D_{0} Z_{0}^{1}\right|_{x_{0}=\underline{x}_{0}},\left.D_{0}^{2} Z_{0}^{1}\right|_{x_{0}=\underline{x}_{0}}$. 
On impose:

$$
\begin{gathered}
Z_{0}\left|=Z_{1}\right|=0 ; \quad \sum_{t} Y_{0}^{t}+Z_{2}\left|=0 ; \quad \sum_{t} Y_{1}^{t}\right|+Z_{3} \mid=0 \\
\ldots ; \quad \sum_{t} Y_{4}^{t}\left|+Z_{6}\right|=I ; \quad \ldots:
\end{gathered}
$$

d'où:

$$
\begin{gathered}
Z_{0}^{1}\left|=0 ; \quad D_{0} Z_{0}^{1}\right|=0 ; \quad b_{04}^{5}|\eta| \sum_{t} Y_{0}^{t 1}\left|+D_{0}^{2} Z_{0}^{1}\right|=0 \\
\sum_{t} D_{0} \psi Y_{0}^{t 1}\left|=0 ; \quad\left(b_{04}^{5}\right)^{2} \sum_{t} Y_{0}^{t 1}\right|=|\eta|^{2} I^{5} ; \quad \ldots ;
\end{gathered}
$$

on obtient les $Y_{0}^{t 1} \mid$ et tous les $Y$; on obtient $D_{0}^{2} Z_{0}^{1} \mid$ et tous les $Z$.

1.6. Cas $(\mathbf{L G})_{5 / 2}$.

$$
\begin{aligned}
f & =\sum_{1 \leq t \leq 5} e^{\psi_{1}^{t}+\psi_{2}^{t}}\left[Y_{0}^{t}+\cdots+Y_{k}^{t}+\cdots\right] \\
\left(D_{0} \psi_{1}^{t}\right)^{5} & =-|\eta|^{4} b_{21}^{5}
\end{aligned}
$$

$\psi_{1}^{t}$ est d'ordre $\frac{2}{5}$.

On a:

$$
D_{0} \psi_{2}^{t}=-|\eta|^{2} \frac{b_{13}^{5}}{\left(D_{0} \psi_{1}^{t}\right)^{2}}
$$

$\psi_{2}^{t}$ est d'ordre $\frac{1}{5} ; Y_{k}^{t}$ est d'ordre $\frac{12-k}{5}$.

(0) (1) (2) $\mathrm{a} Y_{0}=\mathrm{a} Y_{1}=\mathrm{a} Y_{2}=0$;

(3) $\quad|\eta| \mathrm{a} Y_{3}+D_{0} \psi_{1} Y_{0}=0$

(4) $|\eta| \mathrm{a} Y_{4}+D_{0} \psi_{1} Y_{1}+D_{0} \psi_{2} Y_{0}=0$;

(5) $|\eta| \mathrm{a} Y_{5}+D_{0} \psi_{1} Y_{2}+D_{0} \psi_{2} Y_{1}+D_{0} Y_{0}=0$;

(6) (7) (8) (9);

à l'étape (10) apparaît $b_{1} Y_{0}$; à l'étape (15), $b_{2} Y_{0}+\left(D b_{1}\right)_{*}\left(Y_{0}\right)$; on écrit (16) (17); les suivantes détermineront $Y_{1}^{1}$ et ainsi de suite.

On a, en bref:

$$
\begin{array}{ll}
Y_{0}^{2}=Y_{0}^{3}=Y_{0}^{4}=Y_{0}^{5} ; & |\eta| Y_{3}^{2}+D_{0} \psi_{1} Y_{0}^{1}=0 \\
|\eta| Y_{4}^{2}+\cdots+D_{0} \psi_{2} Y_{0}^{1}=0 ; & |\eta| Y_{5}^{2}+\cdots+D_{0} Y_{0}^{1}=0 \\
|\eta| Y_{6}^{3}-\frac{\left(D_{0} \psi_{1}\right)^{2}}{|\eta|} Y_{0}^{1}=0 ; & |\eta| Y_{7}^{3}+\cdots+\frac{2 D_{0} \psi_{1} D_{0} \psi_{2}}{|\eta|} Y_{0}^{1}=0
\end{array}
$$




$$
\begin{aligned}
& |\eta| Y_{8}^{2}+\cdots+\frac{D_{*} \psi_{1}}{|\eta|} D_{0} Y_{0}^{1}+W\left(\psi_{1}, \psi_{2} ;-\frac{3}{5}\right) Y_{0}^{1}=0 \\
& |\eta| Y_{8}^{3}+\cdots-\frac{2 D_{0} \psi_{1}}{|\eta|} D_{0} Y_{0}^{1}+W\left(\psi_{1} ; \psi_{2} ;-\frac{3}{5}\right) Y_{0}^{1}=0 \\
& |\eta| Y_{9}^{4}+\frac{\left(D_{0} \psi_{1}\right)^{3}}{|\eta|^{2}} Y_{0}^{1}=0 ; \quad|\eta| Y_{10}^{4}+\cdots+\frac{3\left(D_{0} \psi_{1}\right)^{2} D_{0} \psi_{2} Y_{0}^{1}}{|\eta|^{2}}=0 \\
& |\eta| Y_{11}^{4}+\cdots+3 \frac{\left(D_{0} \psi\right)^{2}}{|\eta|^{2}} D_{0} Y_{0}^{1}+W\left(\psi_{1}, \psi_{2} ;-\frac{6}{5}\right) Y_{0}^{1}=0 \\
& |\eta| Y_{11}^{3}+\cdots+3 \frac{D_{*} \psi_{1} D_{0} \psi_{1}}{|\eta|^{2}} D_{0} Y_{0}^{1}=0 ; \quad|\eta| Y_{12}^{5}-\frac{\left(D_{0} \psi_{1}\right)^{4}}{|\eta|^{3}} Y_{0}^{1}=0 \\
& |\eta| Y_{13}^{5}-\frac{4\left(D_{0} \psi_{1}\right)^{3}}{|\eta|^{3}} D_{0} \psi_{2} Y_{0}^{1}=0 ; \\
& |\eta| Y_{14}^{5}+\cdots-\frac{4\left(D_{0} \psi_{1}\right)^{3}}{|\eta|^{3}} D_{0} Y_{0}^{1}+W\left(\psi_{1}, \psi_{2} ;-\frac{3}{5}\right) Y_{0}^{1}=0
\end{aligned}
$$

à la $15^{\text {ème }}$ étape, $Y_{0}^{1}$ reste libre compte tenu du choix de $D_{0} \psi_{1}$; à la $16^{\text {ème }}$ étape, $Y_{0}^{1}$ reste libre grâce au choix de $D_{0} \psi_{2}$; à la $17^{\text {ème }}$ étape, on a:

$$
\frac{5\left(D_{0} \psi_{1}\right)^{4}}{|\eta|^{4}} D_{0} Y_{0}^{1}+W\left(\psi_{1} ; \psi_{2} ;-\frac{12}{5}\right) Y_{0}^{1}=0
$$

il reste à déterminer $\left.Y_{0}^{1}\right|_{x_{0}=\underline{x}_{0}}$.

On impose les conditions initiales:

$$
\sum_{t} Y_{0}^{t}\left|=0=\sum_{t} Y_{1}^{t}\right|=\cdots=\sum_{t} Y_{11}^{t}\left|=0 ; \quad \sum_{t} Y_{12}^{t}\right|=I ; \quad \cdots
$$

On en déduit:

$$
\begin{array}{lll}
\sum_{t} Y_{0}^{t 1} \mid=0 ; & \sum_{t} D_{0} \psi_{1}^{t} Y_{0}^{t 1} \mid=0 ; & \sum_{t}\left(D_{0} \psi_{1}^{t}\right)^{2} Y_{0}^{t 1} \mid=0 \\
\sum_{t}\left(D_{0} \psi_{1}^{t}\right)^{3} Y_{0}^{t 1} \mid=0 ; & \left.\sum_{t}\left(D_{0} \psi_{1}^{t}\right)^{4} Y_{0}^{t 1}|=| \eta\right|^{4} I^{5}
\end{array}
$$

On a donc déterminé les $Y_{0}^{t 1}$, puis tous les $Y$.

\subsection{Cas $(\mathbf{L G})_{3}$.}

$$
\begin{aligned}
f & =\sum_{1 \leq t \leq 3} f_{1}^{t}+f_{2} ; \\
f_{1}^{t} & =e^{\psi^{t}}\left[Y_{0}^{t}+\cdots+Y_{k}^{t}+\cdots\right]
\end{aligned}
$$

$\left(D_{0} \psi\right)^{3}=-b_{13}^{5}|\eta|^{2} ; \psi$ est d'ordre $1 / 3 ; Y_{k}$ est d'ordre $\frac{8-k}{3} ;$

$$
f_{2}=Z_{0}+\cdots+Z_{k}+\cdots
$$


$Z_{k}$ est d'ordre $\frac{9-k}{3}$.

On résume la détermination des $Y_{k}$ et des $Z_{k}$.
(0) (1) $\quad \mathrm{a} Y_{0}=\mathrm{a} Y_{1}=0$
(2) $\quad|\eta| \mathrm{a} Y_{2}+D_{0} \psi Y_{0}=0$
$|\eta| \mathrm{a} Y_{3}+D_{0} \psi Y_{1}+D_{0} Y_{0}=0$

à la $6^{\text {ème }}$ étape apparaît $b_{1} Y_{0}$; à la $9^{\text {ème }}$ étape, $b_{2} Y_{0}$.

On a:

$$
\begin{aligned}
& Y_{0}^{2}=Y_{0}^{3}=Y_{0}^{4}=Y_{0}^{5}=\cdots=Y_{1}^{5}=0 \\
& |\eta| Y_{2}^{2}+D_{0} \psi Y_{0}^{1}=0 ; \quad|\eta| Y_{3}^{2}+\cdots+D_{0} Y_{0}^{1}=0 \\
& |\eta| Y_{4}^{2}+\cdots-\frac{D_{*} \psi D_{0} \psi}{|\eta|} Y_{0}^{1}=0 ; \quad|\eta| Y_{4}^{3}-\frac{\left(D_{0} \psi\right)^{2}}{|\eta|} Y_{0}^{1}=0 \\
& |\eta| Y_{5}^{2}-D_{*} \psi \frac{D_{0} Y_{0}^{1}}{|\eta|}-\frac{D_{0} \psi D_{*} Y_{0}^{1}}{|\eta|}+W\left(\psi,-\frac{2}{3}\right) Y_{0}^{1}=0 \\
& |\eta| Y_{5}^{3}+\cdots-2 \frac{D_{0} \psi}{|\eta|} D_{0} Y_{0}^{1}+W\left(\psi,-\frac{2}{3}\right) Y_{0}^{1}=0 \\
& |\eta| Y_{6}^{4}+\frac{\left(D_{0} \psi\right)^{3}}{|\eta|^{3}} Y_{0}^{1}=0 \\
& |\eta| Y_{7}^{4}+\cdots+3 \frac{\left(D_{0} \psi\right)^{2}}{|\eta|} D_{0} Y_{0}^{1}+W\left(\psi ;-\frac{1}{3}\right) Y_{0}^{1}=0 \\
& |\eta| Y_{8}^{5}-\frac{\left(D_{0} \psi\right)^{4}}{|\eta|^{3}} Y_{0}^{1}=0 \\
& |\eta| Y_{9}^{5}+\cdots-4 \frac{\left(D_{0} \psi\right)^{3}}{|\eta|^{2}} D_{0} Y_{0}^{1}+W(\psi ;-1)=0 ; \quad Y_{0}^{1}=0
\end{aligned}
$$

à la $10^{\text {ème }}$ étape, le choix de $D \psi$ laisse $Y_{0}^{1}$ libre; à la $11^{\text {ème }}$ étape, on a:

$$
\begin{gathered}
3 \frac{\left(D_{0} \psi\right)^{4}}{|\eta|^{4}} D_{0} Y_{0}^{1}+W\left(\psi ;-\frac{8}{3}\right) Y_{0}^{1}=0 ; \\
\mathrm{a} Z_{0}=\mathrm{a} Z_{1}=\mathrm{a} Z_{2}=0 ; \quad|\eta| \mathrm{a} Z_{3}+D_{0} Z_{0}=0 ; \\
\ldots ; \quad|\eta| \mathrm{a} Z_{6}+D_{0} Z_{3}+\mathrm{a} D_{1} Z_{3}+b_{1} Z_{0}=0 ;
\end{gathered}
$$

à l'étape (9) apparaît $b_{2} Z_{0}$; à l'étape (12) $b_{3} Z_{0}$. On a:

$$
\begin{array}{ll}
Z_{0}^{2}=Z_{0}^{3}=Z_{0}^{4}=Z_{0}^{5}=0 ; & |\eta| Z_{3}^{2}+D_{0} Z_{0}^{1}=0 ; \\
|\eta| Z_{6}^{3}-\frac{D_{0}^{2} Z_{0}^{1}}{|\eta|}=0 ; & |\eta| Z_{9}^{4}+\frac{D_{0}^{3} Z_{0}^{1}}{|\eta|}=0,
\end{array}
$$

à la $12^{\text {ème }}$ étape, on a:

$$
b_{13}^{5} D_{0}^{2} Z_{0}^{1}-|\eta| b_{22}^{5} D_{0} Z_{0}^{1}+|\eta|^{2} b_{31}^{5} Z_{0}^{1}=0 .
$$


On impose les conditions initiales:

$$
\begin{aligned}
& Z_{0}\left|=0 ; \quad \sum_{t} Y_{0}^{t}\right|+Z_{1}\left|=0 ; \quad \sum_{t} Y_{1}^{t}\right|+Z_{2} \mid=0 ; \quad \ldots \\
& \sum_{t} Y_{7}^{t}\left|+Z_{8}\right|=0 ; \quad \sum_{t} Y_{8}^{t}\left|+Z_{9}\right|=I ; \quad \ldots
\end{aligned}
$$

On a donc:

$$
Z_{0}^{1}\left|=0 ; \quad \sum_{t} D_{0} \psi^{t} Y_{0}^{t 1}\right|+D_{0} Z_{0}^{1}\left|=0 ; \quad \sum_{t}\left(D_{0} \psi^{t}\right)^{2} Y_{0}^{t 1}\right|=0 ; \quad b_{13}^{5} \sum_{t} Y_{0}^{t 1} \mid=0
$$

d'où

$$
\sum_{t} Y_{0}^{t 1}=0 ;\left.\quad b_{13}^{5} \sum_{t} D_{0} \psi^{t} Y_{0}^{t 1}|=-| \eta\right|^{2} I^{5} ; \quad \ldots
$$

d'où les valeurs de $Y_{0}^{t 1} \mid$ et $D_{0} Z_{0}^{1} \mid$ et tous les $Y$ et $Z$.

1.8. Cas $(\mathbf{L G})_{4}$.

$$
\begin{aligned}
f & =\sum_{1 \leq t \leq 4} f_{1}^{t}+f_{2} ; \\
f_{1}^{t} & =e^{\psi^{t}}\left[Y_{0}^{t}+\cdots+Y_{k}^{t}+\cdots\right]
\end{aligned}
$$

$\left(D_{0} \psi^{t}\right)^{4}=b_{22}^{5}|\eta|^{3} ; \psi^{t}$ est d'ordre $1 / 4 ; Y_{k}^{t}$ est d'ordre $\frac{12-k}{4} ;$

$$
f_{2}=Z_{0}+\cdots+Z_{k}+\cdots
$$

$Z_{k}$ est d'ordre $\frac{12-k}{4}$.

On a:

$$
Y_{0}^{2}=Y_{0}^{3}=Y_{0}^{4}=Y_{0}^{5}=0
$$

la $15^{\text {ème }}$ étape s'écrit:

$$
\left[\frac{\left(D_{0} \psi\right)^{5}}{|\eta|^{4}}-b_{22}^{5} \frac{D_{0} \psi}{|\eta|}\right] Y_{0}^{1}=0
$$

et est réalisée sans condition sur $Y_{0}^{1}$; la 16 ème étape donne:

$$
4 \frac{\left(D_{0} \psi\right)^{4}}{|\eta|^{4}} D_{0} Y_{0}^{1}+W(\psi ;-3) Y_{0}^{1}=0
$$


On a aussi:

$$
\begin{aligned}
& \mathrm{a} Z_{0}=\mathrm{a} Z_{1}=\mathrm{a} Z_{2}=\mathrm{a} Z_{3}=0 ; \quad|\eta| \mathrm{a} Z_{4}+D_{0} Z_{0}=0 ; \quad \ldots ; \\
& |\eta| \mathrm{a} Z_{8}+D_{0} Z_{4}+\mathrm{a} D_{*} Z_{4}+b_{1} Z_{0}=0 ; \quad \ldots ; \\
& |\eta| \mathrm{a} Z_{12}+D_{0} Z_{8}+\mathrm{a} D_{*} Z_{8}+b_{1} Z_{4}+b_{2} Z_{0}+\left(D b_{1}\right)_{*} Z_{0}+\sum \mathrm{a} W_{\alpha^{\prime}}(-1) D^{\alpha^{\prime}} Z_{4}=0 \\
& |\eta| \mathrm{a} Z_{16}+D_{0} Z_{12}+\mathrm{a} D_{*} Z_{12}+b_{1} Z_{8}+\left(D b_{1}\right)_{*}\left(Z_{4}\right)+b_{2} Z_{4}+\left(D b_{2}\right)_{*}\left(Z_{0}\right)+b_{3} Z_{0} \\
& \quad+\sum \mathrm{a} W_{\alpha^{\prime}}(-2) D^{\alpha^{\prime}} Z_{4}=0
\end{aligned}
$$

d'où:

$$
Z_{0}^{2}=Z_{0}^{3}=Z_{0}^{4}=Z_{0}^{5}=0 ; \quad b_{22}^{5} D_{0} Z_{0}^{1}-b_{31}^{5}|\eta| Z_{0}^{1}=0
$$

il reste à déterminer les $Y_{0}^{1} \mid$ et $Z_{0}^{1} \mid$. On impose:

$$
\sum_{t} Y_{0}^{t}\left|+Z_{0}\right|=0 ; \quad \ldots ; \quad \sum_{t} Y_{11}^{t}\left|+Z_{11}\right|=0 ; \quad \sum_{t} Y_{12}^{t}\left|+Z_{12}\right|=I ; \quad \ldots
$$

On obtient:

$$
\begin{aligned}
& \sum_{t} Y_{0}^{t 1}\left|+Z_{0}^{1}\right|=0 ; \quad \sum_{t} D_{0} \psi^{t} Y_{0}^{t 1}\left|=0 ; \quad \sum_{t}\left(D_{0} \psi^{t}\right)^{2} Y_{0}^{t 1}\right|=0 \\
& \sum_{t}\left(D_{0} \psi^{t}\right)^{3} Y_{0}^{t 1}\left|=0 ; \quad b_{22}^{5} \sum_{t} Y_{0}^{t 1}\right|=|\eta| I^{5} ; \quad \ldots
\end{aligned}
$$

on obtient tous les $Y_{0}^{t 1} \mid$ et $Z_{0}^{1} \mid$ et tous les $Y$ et $Z$.

1.9. Cas $(\mathbf{L G})_{5}$.

$$
\begin{aligned}
f & =\sum_{1 \leq t \leq 5} f^{t} \\
f^{t} & =e^{\psi^{t}}\left[Y_{0}^{t}+\cdots+Y_{k}^{t}+\cdots\right]
\end{aligned}
$$

$\left(D_{0} \psi^{t}\right)^{5}=-b_{31}^{5}|\eta|^{4} ; \psi^{t}$ est d'ordre $1 / 5 ; Y_{k}^{t}$ est d'ordre $\frac{16-k}{5}$.

$$
\mathrm{a} Y_{0}=\cdots=\mathrm{a} Y_{3}=0 ; \quad|\eta| \mathrm{a} Y_{4}+D_{0} \psi Y_{0}=0 ; \quad|\eta| \mathrm{a} Y_{5}+D_{0} \psi Y_{1}+D_{0} Y_{0}=0
$$

à la $10^{\text {ème }}$ étape apparaît $b_{1} Y_{0}$; à la $15^{\text {ème }}$ étape apparaît $b_{2} Y_{0}+\left(D b_{1}\right)_{*}\left(Y_{0}\right)$ à la $20^{\text {ème }}$ étape apparaît $b_{3} Y_{0}+\left(D b_{2}\right)_{*}\left(Y_{0}\right)$.

On a:

$$
Y_{0}^{2}=Y_{0}^{3}=Y_{0}^{4}=Y_{0}^{5}
$$


à la $20^{\text {ème }}$ étape, on a:

$$
\left[\frac{\left(D_{0} \psi\right)^{5}}{|\eta|^{4}}+b_{31}^{5}\right] Y_{0}^{1}=0
$$

à la $21^{\text {ème }}$ étape:

$$
5 \frac{\left(D_{0} \psi\right)^{4}}{|\eta|^{4}} D_{0} Y_{0}^{1}+W\left(\psi ;-\frac{16}{5}\right) Y_{0}^{1}=0
$$

les conditions initiales imposées sont:

$$
\sum_{t} Y_{0}^{t}\left|=\cdots=\sum_{t} Y_{15}^{t}\right|=0 ; \quad \sum_{t} Y_{16}^{t} \mid=I
$$

d'où:

$$
\begin{array}{ll}
\sum_{t} Y_{0}^{t 1} \mid=0 ; & \sum_{t} D_{0} \psi^{t} Y_{0}^{t 1} \mid=0 ; \quad \ldots \\
\sum_{t}\left(D_{0} \psi^{t}\right)^{3} Y_{0}^{t 1} \mid=0 ; & \left.\sum_{t}\left(D_{0} \psi^{t}\right)^{4} Y_{0}^{t 1}|=| \eta\right|^{4} I^{5}
\end{array}
$$

d'où les $Y_{0}^{t 1} \mid$ et tous les $Y$.

1.10. Cas L. Les conditions L sont toutes satisfaites:

$$
b_{0}=0 ; \quad b_{11}^{5}=b_{12}^{5}=b_{13}^{5}=0 ; \quad b_{21}^{5}=b_{22}^{5}=0 ; \quad b_{31}^{5}=0 .
$$

Le diagramme est vide.

Nous avons ici un cas bien connu, [7] [22]:

$$
f=Y_{0}+\cdots+Y_{k}+\cdots
$$

$Y_{k}$ est d'ordre $4-k$.

On a:

$$
\begin{aligned}
& \mathrm{a} Y_{0}=0 ; \quad|\eta| \mathrm{a} Y_{1}+D_{0} Y_{0}=0 ; \quad|\eta| \mathrm{a} Y_{2}+D_{0} Y_{1}+\mathrm{a} D_{*} Y_{1}+b_{1} Y_{0}=0 \\
& |\eta| \mathrm{a} Y_{3}+D_{0} Y_{2}+\mathrm{a} D_{*} Y_{2}+b_{1} Y_{1}+b_{2} Y_{0}+\left(D b_{1}\right)_{*}\left(Y_{0}\right)+\mathrm{a} \sum_{\left|\alpha^{\prime}\right|=2} W_{\alpha^{\prime}} D^{\alpha^{\prime}} Y_{1}=0 \\
& |\eta| \mathrm{a} Y_{4}+D_{0} Y_{3}+\mathrm{a} D_{*} Y_{3}+b_{1} Y_{2}+b_{2} Y_{1}+b_{3} Y_{0}+\left(D b_{1}\right)_{*}\left(Y_{1}\right)+\left(D b_{2}\right)_{*}\left(Y_{0}\right) \\
& \quad+\mathrm{a} \sum_{\left|\alpha^{\prime}\right|=2} W_{\alpha^{\prime}} D^{\alpha^{\prime}} Y_{2}+\mathrm{a} \sum_{\left|\alpha^{\prime}\right|=3} W_{\alpha^{\prime}} D^{\alpha^{\prime}} Y_{1}=0 ; \\
& \quad+\eta \mid \mathrm{a} Y_{5}+D_{0} Y_{4}+\mathrm{a} D_{*} Y_{4}+b_{1} Y_{3}+b_{2} Y_{2}+b_{3} Y_{1}+b_{4} Y_{0}+\left(D b_{1}\right)_{*}\left(Y_{2}\right) \\
& \quad+\left(D b_{2}\right)_{*}\left(Y_{1}\right)+\left(D b_{3}\right)_{*}\left(Y_{0}\right)+\mathrm{a} \sum_{\left|\alpha^{\prime}\right|=2} W_{\alpha^{\prime}} D^{\alpha^{\prime}} Y_{3} \\
& \quad+\mathrm{a} \sum_{\left|\alpha^{\prime}\right|=3} W_{\alpha^{\prime}} D^{\alpha^{\prime}} Y_{2}+\mathrm{a} \sum_{\left|\alpha^{\prime}\right|=4} W_{\alpha^{\prime}} D^{\alpha^{\prime}} Y_{1}=0 .
\end{aligned}
$$


D'où

$$
\begin{array}{ll}
Y_{0}^{2}=Y_{0}^{3}=Y_{0}^{4}=Y_{0}^{5} ; & |\eta| Y_{1}^{2}+D_{0} Y_{0}^{1}=0 ; \quad Y_{1}^{3}=Y_{1}^{4}=Y_{1}^{5}=0 \\
|\eta| Y_{2}^{3}-\frac{D_{0}^{2} Y_{0}^{1}}{|\eta|}=0 ; & Y_{2}^{4}=Y_{2}^{5}=0 \\
|\eta| Y_{3}^{4}+\frac{D_{0}^{3} Y_{0}^{1}}{|\eta|^{2}}=0 ; & Y_{3}^{5}=0 ; \quad|\eta| Y_{4}^{5}-\frac{D_{0}^{4} Y_{0}^{1}}{|\eta|^{3}}=0
\end{array}
$$

à l'étape (5), on a:

$$
D_{0}^{5} Y_{0}^{5}+W(0) D_{0}^{3} Y_{0}^{1}+W^{\prime}(0) Y_{0}^{1}+W^{\prime \prime}(0) D_{0} Y_{0}^{1}+W^{\prime \prime \prime}(0) Y_{0}^{1}=0 .
$$

On impose les conditions initiales:

$$
Y_{0}\left|=Y_{1}\right|=Y_{2}\left|=Y_{3}\right|=0 ; \quad Y_{4} \mid=I ; \quad \ldots ;
$$

d'où

$$
Y_{0}^{1}\left|=D_{0} Y_{0}^{1}\right|=D_{0}^{2} Y_{0}^{1}\left|=D_{0}^{3} Y_{0}^{1}\right|=0 ;\left.\quad D_{0}^{4} Y_{0}^{1}|=| \eta\right|^{4} I^{5} ; \quad \ldots
$$

d'où $Y_{0}^{1}$ et tous les $Y_{k}$.

\section{§2. Étude du type $(4,1)$}

$$
h=\left(\begin{array}{ccccc}
D_{0} & \left|D^{\prime}\right| & 0 & 0 & 0 \\
0 & D_{0} & \left|D^{\prime}\right| & 0 & 0 \\
0 & 0 & D_{0} & \left|D^{\prime}\right| & 0 \\
b_{1}^{4} & b_{2}^{4} & b_{3}^{4} & D_{0} & b_{5}^{4} \\
b_{1}^{5} & 0 & 0 & 0 & D_{0}
\end{array}\right)
$$

Les conditions $(\mathrm{LG})_{d}$ s'écrivent:

$$
\begin{aligned}
&(\mathrm{LG})_{4 / 3}: b_{01}^{4} \neq 0 ; \\
&(\mathrm{LG})_{3 / 2}: b_{01}^{4}=0 ; \quad b_{02}^{4} \neq 0 ; \\
&(\mathrm{LG})_{5 / 3}: b_{01}^{4}=b_{02}^{4}=0 ; \quad b_{05}^{4} b_{01}^{5} \neq 0 ; \\
&(\mathrm{LG})_{2}: b_{01}^{4}=b_{02}^{4}=b_{05}^{4} b_{01}^{5}=0 ; \quad b_{03}^{4} \text { ou } b_{11}^{4} \neq 0 ; \\
&(\mathrm{LG})_{5 / 2}: b_{01}^{4}=b_{02}^{4}=b_{03}^{4}=b_{05}^{4} b_{01}^{5}=b_{11}^{4}=0, \quad b_{15}^{4} b_{01}^{5}+b_{05}^{4} b_{11}^{5} \neq 0 ; \\
&(\mathrm{LG})_{3}: b_{01}^{4}=b_{02}^{4}=b_{03}^{4}=b_{05}^{4} b_{01}^{5}=b_{11}^{4}=b_{15}^{4} b_{01}^{5}+b_{05}^{4} b_{11}^{5}=0, \quad b_{12}^{4} \neq 0 \\
&(\mathrm{LG})_{4}: b_{01}^{4}=b_{02}^{4}=b_{03}^{4}=b_{05}^{4} b_{01}^{5}=b_{11}^{4}=b_{12}^{4}=b_{15}^{4} b_{01}^{5}+b_{05}^{4} b_{11}^{5}=0, \quad b_{21}^{4} \neq 0 \\
&(\mathrm{LG})_{5}: b_{01}^{4}=b_{02}^{4}=b_{03}^{4}=b_{05}^{4} b_{01}^{5}=b_{11}^{4}=b_{12}^{4}=b_{15}^{4} b_{01}^{5}+b_{05}^{4} b_{11}^{5}=b_{21}^{4}=0 \\
& b_{15}^{4} b_{11}^{5}+b_{25}^{4} b_{01}^{5}+b_{05}^{4} b_{21}^{5} \neq 0
\end{aligned}
$$


Les conditions L s'écrivent:

$$
\begin{aligned}
b_{01}^{4}=b_{02}^{4}=b_{03}^{4}=b_{05}^{4} b_{01}^{5} & =b_{11}^{4}=b_{12}^{4}=b_{15}^{4} b_{01}^{5}+b_{05}^{4} b_{11}^{5} \\
& =b_{21}^{4}=b_{15}^{4} b_{11}^{5}+b_{25}^{4} b_{01}^{5}+b_{05}^{4} b_{21}^{5}=0
\end{aligned}
$$

Notations. Nous noterons simplement $W$ les termes polynomiaux en dérivées des $\psi$ qu'il n'est pas nécessaire de préciser.

2.1. Cas $(\mathbf{L G})_{4 / 3}$. $N$ est formé de points $0,(4,3)$ et $(5,3)$. On cherche donc $f$ sous la forme:

$$
\begin{aligned}
f & =\sum_{1 \leq t \leq 4} f_{1}^{t}+f_{2} \\
f_{1}^{t} & =e^{\psi_{1}^{t}+\psi_{2}^{t}+\psi_{3}^{t}}\left[Y_{0}^{t}+\cdots+Y_{k}^{t}+\cdots\right]
\end{aligned}
$$

les 4 phases complexes $\psi_{1}^{t}$ distinctes, $1 \leq t \leq 4$, vérifient:

$$
\left(D_{0} \psi_{1}^{t}\right)^{4}=b_{01}^{4}|\eta|^{3} \neq 0 ; \quad \psi_{1}^{t} \mid=0 ;
$$

$\psi_{1}^{t}$ est d'ordre $3 / 4$

$D_{0} \psi_{2}^{t}=\frac{1}{4|\eta|}\left[3 D_{0} \psi_{1}^{t} D_{*} \psi_{1}^{t}-\frac{b_{02}^{4}}{b_{01}^{4}}\left(D_{0} \psi_{1}^{t}\right)^{2}-|\eta| \sum_{\left|\alpha^{\prime}\right|=2} \frac{1}{\alpha^{\prime \prime} !} \partial_{\alpha^{\prime}}|\eta|\left(D \psi_{1}\right)^{\alpha^{\prime}}\right] ; \quad \psi_{2}^{t} \mid=0$

$\psi_{2}^{t}$ est d'ordre $1 / 2 ; D_{0} \psi_{3}^{t}$ s'exprime de façon analogue en fonction de $\psi_{1}^{t}$ et $\psi_{2}^{t}$ et est d'ordre $1 / 4$. $Y_{k}^{t}$ est d'ordre $\frac{3-k}{4}$;

$$
f_{2}=Z_{0}+\cdots Z_{k}+\cdots
$$

$Z_{k}$ est d'ordre $-k / 4$.

Les systèmes en $Y$ et $Z$ sont les mêmes que dans le $§ 1.2$. On obtient ici:

(0) $Y_{0}^{2}=Y_{0}^{3}=Y_{0}^{4}=0$;

(1) $|\eta| Y_{1}^{2}+D_{0} \psi_{1} Y_{0}^{1}=0, \quad Y_{1}^{3}=Y_{1}^{4}=0, \quad Y_{0}^{5}=0$;

(2) $|\eta| Y_{2}^{2}+\cdots+D_{0} \psi_{2} Y_{0}^{1}-\frac{D_{*} \psi_{1} D_{0} \psi_{1}}{|\eta|} Y_{0}^{1}=0$

$$
|\eta| Y_{2}^{3}-\frac{\left(D_{0} \psi_{1}\right)^{2}}{|\eta|} Y_{0}^{1}=0, \quad Y_{2}^{4}=0, \quad Y_{1}^{5}=0 ;
$$


(3) $|\eta| Y_{3}^{2}+\cdots+\left[D_{0} \psi_{3}-\frac{2 D_{*} \psi_{1} D_{0} \psi_{2}}{|\eta|}+\frac{\left(D_{*} \psi_{1}\right)^{2} D_{0} \psi_{1}}{|\eta|^{2}}\right.$

$$
\begin{gathered}
\left.-\sum_{\left|\alpha^{\prime}\right|=2} \frac{1}{\alpha !} \partial_{\alpha^{\prime}}|\eta|\left(D \psi_{1}\right)^{\alpha^{\prime}} \frac{1}{|\eta|}\right] Y_{0}^{1}=0 \\
|\eta| Y_{3}^{3}+\cdots+\left[-\frac{2 D_{0} \psi_{1} D_{0} \psi_{2}}{|\eta|}+2 \frac{\left(D_{0} \psi_{1}\right)^{2} D_{*} \psi_{1}}{|\eta|^{2}}\right] Y_{0}^{1}=0
\end{gathered}
$$$$
|\eta| Y_{3}^{4}+\frac{\left(D_{0} \psi_{1}\right)^{3}}{|\eta|^{2}} Y_{0}^{1}=0, \quad Y_{2}^{5}=0
$$

(4) $|\eta| Y_{4}^{2}+\cdots+D_{0} Y_{0}^{1}+W Y_{0}^{1}=0$;

$$
\begin{aligned}
& |\eta| Y_{4}^{3}+\cdots+\left[-\frac{2 D_{0} \psi_{1} D_{0} \psi_{3}}{|\eta|}+\frac{4 D_{0} \psi_{1} D_{*} \psi_{1} D_{0} \psi_{2}}{|\eta|^{2}}-\frac{3\left(D_{*} \psi_{1}\right)^{2}\left(D_{0} \psi_{1}\right)^{2}}{|\eta|^{3}}\right. \\
& \left.-\frac{\left(D_{0} \psi_{2}\right)^{2}}{|\eta|}+\frac{D_{*} \psi_{2}\left(D_{0} \psi_{1}\right)^{2}}{|\eta|^{2}}+\sum_{\left|\alpha^{\prime}\right|=2} \frac{1}{\alpha !} \frac{\partial_{\alpha^{\prime}}|\eta|\left(D \psi_{1}\right)^{\alpha^{\prime}}}{|\eta|^{2}}\left(D_{0} \psi_{1}\right)^{2}\right] Y_{0}^{1}=0 \\
& |\eta| Y_{4}^{4}+\cdots+\left[\frac{3\left(D_{0} \psi_{1}\right)^{2} D_{0} \psi_{2}}{|\eta|^{2}}-\frac{3\left(D_{0} \psi_{1}\right)^{3} D_{*} \psi_{1}}{|\eta|^{3}}\right] Y_{0}^{1}=0 \\
& {\left[-\frac{\left(D_{0} \psi_{1}\right)^{4}}{|\eta|^{3}}+b_{01}^{4}\right] Y_{0}^{1}=0}
\end{aligned}
$$

qui est réalisée en laissant $Y_{0}^{1}$ libre

$$
D_{0} \psi_{1} Y_{3}^{5}+b_{01}^{5} Y_{0}^{1}=0
$$

(5) $|\eta| Y_{5}^{3}+\cdots-\frac{2\left(D_{0} \psi_{1}\right)^{2} D_{0} Y_{0}^{1}}{|\eta|^{2}}+W Y_{0}^{1}=0$;

nous laissons la détermination de $Y_{5}^{4}$ au lecteur.

La $4^{\text {ème }}$ ligne laisse $Y_{0}^{1}$ libre compte tenu du choix de $D_{0} \psi_{2}$.

(6) $|\eta| Y_{6}^{4}+\cdots+3 \frac{\left(D_{0} \psi_{1}\right)^{2}}{|\eta|^{2}} D_{0} Y_{0}^{1}+W Y_{0}^{1}=0$.

La $4^{\text {ème }}$ ligne laisse $Y_{0}^{1}$ libre compte tenu du choix de $D_{0} \psi_{3}$.

On obtient:

(7) $\quad-\frac{4\left(D_{0} \psi_{1}\right)^{3}}{|\eta|^{3}} D_{0} Y_{0}^{1}+W Y_{0}^{1}=0$

Pour $Z$, on obtient:
(0) $Z_{0}^{2}=Z_{0}^{3}=Z_{0}^{4}=0$;
(1) $\ldots$;
(2) $\ldots$;
(3) $\ldots$;
(4) $\quad D_{0} Z_{0}^{5}+b_{01}^{5} Z_{0}^{1}=0, \quad b_{01}^{4} Z_{0}^{1}+b_{05}^{4} Z_{0}^{5}=0$;

d'où

$$
D_{0} Z_{0}^{5}-\frac{b_{05}^{4} b_{01}^{5}}{b_{01}^{4}} Z_{0}^{5}=0
$$


On impose les conditions initiales:

$$
\sum_{t} Y_{0}^{t}\left|=\sum_{t} Y_{1}^{t}\right|=\sum_{t} Y_{2}^{t}\left|=\sum_{t} Y_{3}^{t}\right|+Z_{0} \mid=I
$$

on en déduit:

$$
\begin{aligned}
\sum_{t} Y_{0}^{t 1} \mid & =0=\sum_{t} D_{0} \psi_{1}^{t} Y_{0}^{t 1}\left|=\sum_{t}\left(D_{0} \psi_{1}^{t}\right)^{2} Y_{0}^{t 1}\right| ;\left.\quad \sum_{t}\left(D_{0} \psi_{1}^{t}\right)^{3} Y_{0}^{t 1}|=| \eta\right|^{3} I^{4} \\
& -\left.\left.\frac{b_{01}^{5}}{b_{01}^{4}} \sum_{t}\left(D_{0} \psi_{1}^{t}\right)^{3} Y_{0}^{t 1}|+| \eta\right|^{3} Z_{0}^{5}|=| \eta\right|^{3} I^{5} ; \quad Z_{0}^{5}\left|=\frac{b_{01}^{5}}{b_{01}^{4}}\right| I^{4}+I^{5} ;
\end{aligned}
$$

d'où tous les coefficients.

\subsection{Cas $(\mathbf{L G})_{3 / 2}$.}

a) $\quad b_{05}^{4} b_{01}^{5} \neq 0 . \quad N$ est formé de points $0,(3,2),(5,3)$. De même qu'au $\S 1.3$, on cherche $f$ sous la forme: (on omettra de réécrire: $\psi_{1}^{t} \mid=0, \ldots$ )

$$
\begin{aligned}
f= & \sum_{1 \leq t \leq 3} f_{1}^{t}+\sum_{1 \leq t^{\prime} \leq 2} f_{2}^{t^{\prime}} ; \\
f_{1}^{t}= & e^{\psi_{1}^{t}+\psi_{2}^{t}}\left[Y_{0}^{t}+\cdots+Y_{k}^{t}+\cdots\right] ; \\
& \left(D_{0} \psi_{1}^{t}\right)^{3}=-b_{02}^{4}|\eta|^{2},
\end{aligned}
$$

$\psi_{1}^{t}$ est d'ordre $2 / 3 ; D_{0} \psi_{2}^{t}$ s'exprime à l'aide de $D_{0} \psi_{1}^{t}$ et est d'ordre $1 / 3 ; Y_{k}^{t}$ est d'ordre: $\frac{6-k}{6}$.

$$
f_{2}^{t^{\prime}}=e^{\psi^{t^{\prime}}}\left[Z_{0}^{t^{\prime}}+\cdots+Z_{k}^{t^{\prime}}+\cdots\right] ; \quad\left(D_{0} \chi^{t^{\prime}}\right)^{2}=-\frac{b_{05}^{4} b_{01}^{5}}{b_{02}^{4}}|\eta|
$$

$\chi^{t^{\prime}}$ est d'ordre $\frac{1}{2} ; Z_{k}^{t^{\prime}}$ est d'ordre: $\frac{6-k}{6}$.

Les systèmes en $Y$ et $Z$ sont ceux du $\S 1.3$. Les calculs sont analogues et nous les résumerons:

(0) $Y_{0}^{2}=Y_{0}^{3}=Y_{0}^{4}=0$;

(2) $Y_{0}^{5}=0$

$|\eta| Y_{2}^{2}+D_{0} \psi_{1} Y_{0}^{1}=0 ; \ldots$

(3) $\ldots$;

(4) $|\eta| Y_{4}^{3}-\frac{\left(D_{0} \psi_{1}\right)^{2}}{|\eta|} Y_{0}^{1}=0, \quad \ldots$

(5) $\ldots$;

(6) $|\eta| Y_{6}^{2}+\cdots+D_{0} Y_{0}^{1}+W Y_{0}^{1}=0, \quad \ldots, \quad|\eta| Y_{6}^{4}+\frac{\left(D_{0} \psi_{1}\right)^{3}}{|\eta|^{2}} Y_{0}^{1}=0$

(7) $\ldots$;

(8) $|\eta| Y_{8}^{3}+\cdots-\frac{2 D_{0} \psi_{1}}{|\eta|} D_{0} Y_{0}^{1}+W Y_{0}^{1}=0, \quad \cdots$

la $4^{\text {ème }}$ ligne laisse $Y_{0}^{1}$ libre par le choix de $D_{0} \psi_{1}$.
(9) $\ldots$;
(10) $|\eta| Y_{10}^{4}+\cdots+\frac{3\left(D_{0} \psi_{1}\right)^{2}}{|\eta|^{2}} D_{0} Y_{0}^{1}+W Y_{0}^{1}=0$ 
la $4^{\text {ème }}$ ligne laisse $Y_{0}^{1}$ libre par le choix de $D_{0} \psi_{2}$.

$$
\begin{aligned}
& \text { (11) } \quad \ldots ; \quad(12) \quad-\frac{3\left(D_{0} \psi_{1}\right)^{3}}{|\eta|^{3}} D_{0} Y_{0}^{1}+W Y_{0}^{1}=0 \\
& \text { (0) } Z_{0}^{2}=Z_{0}^{3}=Z_{0}^{4}=0 ; \quad(1) \quad \ldots ; \quad(2) \quad \ldots ; \\
& \text { (3) }|\eta| Z_{3}^{2}+D_{0} \chi Z_{0}^{1}=0, \quad Z_{0}^{5}=0 ; \quad(4) \quad \ldots ; \quad(5) \quad \ldots ; \\
& \text { (6) }|\eta| Z_{6}^{2}+\cdots+D_{0} Z_{0}^{1}+W Z_{0}^{1}=0 ; \quad|\eta| Z_{6}^{3}-\frac{\left(D_{0} \chi\right)^{2}}{|\eta|} Z_{0}^{1}=0 ; \\
& \\
& D_{0} \chi Z_{3}^{5}+b_{01}^{5} Z_{0}^{1}=0 ;
\end{aligned}
$$

(7) $\ldots ;$; (8) $\ldots ;$ (9) la $4^{\text {ème }}$ ligne laisse $Z_{0}^{1}$ libre par le choix de $D_{0} \chi$;

$$
D_{0} \chi Z_{0}^{5}+\cdots-\frac{b_{01}^{5}}{D_{0} \chi} D_{0} Z_{0}^{1}+W Z_{0}^{1}=0
$$

(12) On obtient à la $4^{\text {ème }}$ ligne:

$$
-\frac{2 b_{02}^{4}}{|\eta|} D_{0} Z_{0}^{1}+W Z_{0}^{1}=0
$$

On impose les conditions initiales.

$$
\begin{aligned}
& \sum_{t} Y_{0}^{1}\left|+\sum_{t^{\prime}} Z_{0}^{t^{\prime}}\right|=0 ; \ldots \sum_{t} Y_{5}^{t}\left|+\sum_{t^{\prime}} Z_{5}^{t^{\prime}}\right|=0 ; \quad \sum_{t} Y_{6}^{t}\left|+\sum_{t^{\prime}} Z_{6}^{t^{\prime}}\right|=I ; \quad \ldots \\
& \text { (0) donne } \sum_{t} Y_{0}^{t 1}\left|+\sum_{t^{\prime}} Z_{0}^{t^{\prime} 1}\right|=0 \text {; } \\
& \text { (3) } \sum_{t^{\prime}} D_{0} \chi^{t^{\prime}} Z_{0}^{t^{\prime} 1} \mid=0 \text {; } \\
& \text { (4) } \quad \sum_{t}\left(D_{0} \psi_{1}^{t}\right)^{2} Y_{0}^{t 1} \mid=0 \\
& \text { (2) } \sum_{t} D_{0} \psi_{1}^{t} Y_{0}^{t 1} \mid=0 \\
& \text { (6) } \sum_{t} Y_{0}^{t 1}|=| \eta\left|b_{02}^{4}\right| I^{4}
\end{aligned}
$$

d'où les $Y_{0}^{t 1} \mid$, puis $\sum_{t^{\prime}} Z_{0}^{t^{\prime} 1} \mid$ et les $Z_{0}^{t^{\prime} 1} \mid$; on en déduit les $Y_{0}^{t}, Z_{0}^{t^{\prime}}$ et tous les coefficients.

b) $\quad b_{05}^{4} b_{01}^{5}=0 . \quad N$ est formé des points $0,(3,2),(4,2),(5,2)$. De même qu'au $\S 1.3$, on cherche $f$ sous la forme:

$$
\begin{aligned}
f & =\sum_{1 \leq t \leq 3} f_{1}^{t}+f_{2} \\
f_{1}^{t} & =e^{\psi_{1}^{t}+\psi_{2}^{t}}\left[Y_{0}^{t}+\cdots+Y_{k}^{t}+\cdots\right]
\end{aligned}
$$

$\left(D_{0} \psi_{1}^{t}\right)^{3}=-b_{02}^{4}|\eta|^{2}, \psi_{1}^{t}$ est d'ordre $2 / 3 ; \psi_{2}^{t}$ est d'ordre $1 / 3 ; Y_{k}^{t}$ est d'ordre $\frac{3-k}{3} ;$

$$
f_{2}=Z_{0}+\cdots+Z_{k}+\cdots
$$


$Z_{k}$ est d'ordre $\frac{3-k}{3}$.

Les systèmes en $Y$ et $Z$ sont ceux du $§ 1.3$.

On a:

$$
Y_{0}^{2}=Y_{0}^{3}=Y_{0}^{4}=Y_{0}^{5}=0
$$

à la $4^{\text {ème }}$ étape, le choix de $D_{0} \psi_{1}$ laisse $Y_{0}^{1}$ libre; à la $5^{\text {ème }}$ étape, le choix de $D_{0} \psi_{2}$ laisse $Y_{0}^{1}$ libre; à la $6^{\text {ème }}$ étape, on obtient:

$$
-\frac{3\left(D_{0} \psi_{1}\right)^{3}}{|\eta|^{2}} D_{0} Y_{0}^{1}+W Y_{0}^{1}=0
$$

Par le système en $Z$, on doit distinguer 2 sous cas.

1) $b_{05}^{4} \neq 0, b_{01}^{5}=0$. On a:

$$
Z_{0}^{2}=Z_{0}^{3}=Z_{0}^{4}=Z_{0}^{5}=0, \quad \frac{b_{02}^{4}}{b_{05}^{4}} D_{0}^{2} Z_{0}^{1}+W D_{0} Z_{0}^{1}+W^{\prime} Z_{0}^{1}=0 .
$$

2) $b_{05}^{4}=0$.

$$
Z_{0}^{2}=Z_{0}^{3}=Z_{0}^{4}=0 ; \quad-\frac{b_{02}^{4}}{|\eta|} D_{0} Z_{0}^{1}+W Z_{0}^{1}+W^{\prime} Z_{0}^{5}=0 ; \quad D_{0} Z_{0}^{5}+b_{01}^{5} Z_{0}^{1}=0
$$

On impose les conditions initiales:

$$
\sum_{t} Y_{0}^{t}\left|+Z_{0}\right|=0=\sum_{t} Y_{1}^{t}\left|+Z_{1}\right|=\sum_{t} Y_{2}^{t}\left|+Z_{2}\right| ; \quad \sum_{t} Y_{3}^{t}\left|+Z_{3}\right|=I
$$

1) $b_{05}^{4} \neq 0$. On obtient:

$$
\begin{aligned}
& \sum_{t} Y_{0}^{t 1}\left|+Z_{0}^{1}\right|=0 ; \quad \sum_{t} D_{0} \psi_{1} Y_{0}^{t 1} \mid=0 ; \\
& \sum_{t}\left(D_{0} \psi_{1}^{t}\right)^{2} Y_{0}^{t 1}\left|=0 ; \quad \sum_{t} Y_{0}^{t 1}\right|=\frac{|\eta|}{b_{02}^{4} \mid} I^{4}
\end{aligned}
$$

d'où les $Y_{0}^{t 1} \mid$ et $Z_{0}^{1} \mid$ et tous les coefficients.

2) $b_{05}^{4}=0$.

$$
\begin{aligned}
& \sum_{t} Y_{0}^{t 1}\left|+Z_{0}^{1}\right|=0, \quad Z_{0}^{5}\left|=0 ; \quad \sum_{t} D_{0} \psi_{1}^{t} Y_{0}^{t 1}\right|=0 ; \\
& \sum_{t}\left(D_{0} \psi_{1}^{t}\right)^{2} Y_{0}^{t 1}\left|=0 ; \quad \sum_{t} Y_{0}^{t 1}\right|=\frac{|\eta|}{b_{02}^{4} \mid} I^{4}
\end{aligned}
$$

d'où les $Y_{0}^{t 1}\left|, Z_{0}^{1}\right|$ et tous les coefficients. 
2.3. Cas $(\mathbf{L G})_{5 / 3}$. On cherche $f$ sous la forme:

$$
\begin{aligned}
f & =\sum_{1 \leq t \leq 5} f_{1}^{t} ; \\
f_{1}^{t} & =e^{\psi_{1}^{t}+\psi_{2}^{t}+\psi_{3}^{t}}\left[Y_{0}^{t}+\cdots+Y_{k}^{t}+\cdots\right]
\end{aligned}
$$

$\left(D_{0} \psi_{1}^{t}\right)^{5}=|\eta|^{3} b_{05}^{4} b_{01}^{5} ; \psi_{1}^{t}$ est d'ordre $3 / 5 ; D_{0} \psi_{2}$ s'exprime à l'aide de $D_{0} \psi_{1}^{t}$, et $\psi_{2}^{t}$ est d'ordre $2 / 5 ; D_{0} \psi_{3}$ s'exprime à l'aide de $D_{0} \psi_{1}^{t}, D_{*} \psi_{1}^{t}$ et $D_{0} \psi_{2}^{t} ; \psi_{3}^{t}$ est d'ordre $1 / 5 ; Y_{k}^{t}$ est d'ordre $\frac{6-k}{5}$.

En résumé:

$$
Y_{0}^{2}=Y_{0}^{3}=Y_{0}^{4}=Y_{0}^{5}=0:
$$

à la $11^{\text {ème }}$ étape, on a l'équation de propagation:

$$
-\frac{\left(D_{0} \psi_{1}\right)^{3}}{|\eta|^{3}} D_{0} Y_{0}^{1}+W Y_{0}^{1}=0
$$

On impose les conditions initiales:

$$
\sum_{t} Y_{0}^{t}\left|=0=\cdots=\sum_{t} Y_{5}^{t}\right| ; \quad \sum_{t} Y_{6}^{t} \mid=I ; \quad \cdots ;
$$

on en déduit:

$$
\begin{aligned}
& \sum_{t} Y_{0}^{t 1}\left|=0=D_{0} \psi_{1}^{t} Y_{0}^{t 1}\right|=\sum_{t}\left(D_{0} \psi_{1}^{t}\right)^{4} Y_{0}^{t 1}\left|=\sum_{t}\left(D_{0} \psi_{1}^{t}\right)^{2} Y_{0}^{t 1}\right| \\
& \left.\sum_{t}\left(D_{0} \psi_{1}^{t}\right)^{3} Y_{0}^{t 1}|=-| \eta\right|^{3} I^{3}
\end{aligned}
$$

d'où les $Y_{0}^{t 1}$ et tous les coefficients.

2.4. Cas $(\mathbf{L G})_{2}$. Ce cas est analogue au cas $d u \S 1.5$.

1) $\quad b_{11}^{4} \neq 0$. Le premier segment de $N$ contient les points: $0,(2,1),(4,2)$

1a) On suppose de plus: $\left(b_{03}^{4}\right)^{2}+4 b_{11}^{4}|\eta| \neq 0$

$$
\begin{aligned}
f & =\sum_{1 \leq t \leq 4} f_{1}^{t}+f_{2} \\
f_{1}^{t} & =e^{\psi^{t}}\left[Y_{0}^{t}+\cdots+Y_{k}^{t}+\cdots\right]
\end{aligned}
$$

$D_{0} \psi^{t}$ est l'une des quatre racines distinctes non nulles de l'équation:

$$
\left(D_{0} \psi\right)^{4}-b_{03}^{4}|\eta|\left(D_{0} \psi\right)^{2}-b_{11}^{4}|\eta|^{3}=0
$$


$\psi^{t}$ est d'ordre $1 / 2 ; Y_{k}^{t}$ est d'ordre: $\frac{3-k}{2}$;

$$
f_{2}=Z_{0}+\cdots+Z_{k}+\cdots
$$

$Z_{k}$ est d'ordre $\frac{2-k}{2}$.

Les systèmes en $Y$ et $Z$ sont ceux du $§ 1.5$, Cas 1a). On obtient, en résumé:

(0) $Y_{0}^{2}=Y_{0}^{3}=Y_{0}^{4}=0$;

(1) $|\eta| Y_{1}^{2}+D_{0} \psi Y_{0}^{1}=0, \quad Y_{0}^{5}=0$;

(2) $|\eta| Y_{2}^{2}+\cdots+D_{0} Y_{0}^{1}-\frac{D_{*} \psi D_{0} \psi}{|\eta|} Y_{0}^{1}=0$,

$$
|\eta| Y_{2}^{3}-\frac{\left(D_{0} \psi\right)^{2}}{|\eta|} Y_{0}^{1}=0, \quad D_{0} \psi Y_{1}^{5}+b_{01}^{5} Y_{0}^{1}=0
$$

(3) $|\eta| Y_{3}^{2}+\cdots-\frac{D_{*} \psi}{|\eta|} D_{0} Y_{0}^{1}-\frac{D_{0} \psi}{|\eta|}+D_{*} Y_{0}^{1}+W Y_{0}^{1}=0$

$$
\begin{aligned}
& |\eta| Y_{3}^{3}-\frac{2 D_{0} \psi}{|\eta|} D_{0} Y_{0}^{1}+\left[2 \frac{\left(D_{0} \psi\right)^{2} D_{*} \psi}{|\eta|^{2}}-\frac{D_{0}^{2} \psi}{|\eta|}\right] Y_{0}^{1}=0, \\
& |\eta| Y_{3}^{4}+\frac{\left(D_{0} \psi\right)^{3}}{|\eta|^{2}} Y_{0}^{1}=0 \\
& D_{0} \psi Y_{2}^{5}+\cdots-\frac{b_{01}^{5}}{D_{0} \psi} D_{0} Y_{0}^{1}+\left[b_{01}^{5} \frac{D_{0}^{2} \psi}{\left(D_{0} \psi\right)^{2}}-\frac{D_{0} b_{01}^{5}}{D_{0} \psi}+\left(D b_{1}^{5}\right)_{*}(\psi)\right] Y_{0}^{1}=0
\end{aligned}
$$

(4) La $4^{\text {ème }}$ ligne laisse $Y_{0}^{1}$ libre compte tenu du choix de $D_{0} \psi$.

$$
\begin{aligned}
& |\eta| Y_{4}^{3}+\cdots-\frac{D_{0}^{2} Y_{0}^{1}}{|\eta|}+2 \frac{\left(D_{0} \psi\right)^{2}}{|\eta|^{2}} D_{*} Y_{0}^{1}+W D_{0} Y_{0}^{1}+W^{\prime} Y_{0}^{1}=0 \\
& |\eta| Y_{4}^{4}+\cdots+\frac{3\left(D_{0} \psi\right)^{2}}{|\eta|^{2}} D_{0} Y_{0}^{1}+\left[-\frac{3\left(D_{0} \psi\right)^{3} D_{0} \psi}{|\eta|^{3}}+\frac{3 D_{0} \psi D_{0}^{2} \psi}{|\eta|^{2}}\right] Y_{0}^{1}=0 \\
& D_{0} \psi Y_{3}^{5}+\cdots+\frac{b_{01}^{5}}{\left(D_{0} \psi\right)^{2}} D_{0}^{2} Y_{0}^{1}+W D_{0} Y_{0}^{1}+W^{\prime} Y_{0}^{1}=0 \\
& \quad-\frac{2 D_{0} \psi}{|\eta|}\left[2\left(D_{0} \psi\right)^{2}-b_{03}^{4}|\eta|\right] D_{0} Y_{0}^{1}+W Y_{0}^{1}=0
\end{aligned}
$$

En $Z$ :
(0) $Z_{0}^{2}=Z_{0}^{3}=Z_{0}^{4}=0$;
(1) $\ldots$
(2) $\quad b_{05}^{4} Z_{0}^{5}=0$
$D_{0} Z_{0}^{5}+b_{01}^{5} Z_{0}^{1}=0$

On distingue deux cas:

i) $b_{05}^{4} \neq 0$. Alors: $Z_{0}^{5}=0, \ldots$

$$
\text { (4) } D_{0} Z_{2}^{5}+b_{11}^{5} Z_{0}^{1}=0, \quad b_{05}^{4} Z_{2}^{5}+b_{11}^{4} Z_{0}^{1}=0,
$$

d'où:

$$
D_{0} Z_{0}^{1}+W Z_{0}^{1}=0
$$


ii) $b_{05}^{4}=0$.

$$
b_{11}^{4} Z_{0}^{1}+b_{15}^{4} Z_{0}^{5}=0
$$

d'où:

$$
D_{0} Z_{0}^{5}+W Z_{0}^{5}=0
$$

On impose les conditions initiales:

$$
\begin{array}{ll}
\sum_{t} Y_{0}^{t} \mid=0 ; & \sum_{t} Y_{1}^{t}\left|+Z_{0}\right|=0 ; \\
\sum_{t} Y_{2}^{t}\left|+Z_{1}\right|=0 ; & \sum_{t} Y_{3}^{t}\left|+Z_{2}\right|=I ; \quad \ldots ;
\end{array}
$$

on obtient, si $b_{05}^{4} \neq 0$ :

$$
\begin{array}{ll}
\sum_{t} Y_{0}^{t 1} \mid=0 ; & \sum_{t} D_{0} \psi^{t} Y_{0}^{t 1} \mid=0 \\
\sum_{t}\left(D_{0} \psi^{t}\right)^{2} Y_{0}^{t 1} \mid=0 ; & \left.\sum_{t}\left(D_{0} \psi^{t}\right)^{3} Y_{0}^{t 1}|=-| \eta\right|^{3} I^{4}
\end{array}
$$

d'où les $Y_{0}^{t 1} \mid$;

$$
b_{11}^{4} Z_{0}^{1}\left|=-b_{05}^{4} b_{11}^{5} \sum_{t} \frac{Y_{0}^{t 1}}{D_{0} \psi^{t}}\right|-b_{05}^{4} \mid I^{5}
$$

d'où $Z_{0}^{1} \mid$; on obtient: les $Y_{0}^{t 1}$ et $Z_{0}^{1}$ et tous les coefficients.

Si $b_{05}^{4}=0$;

$$
\begin{array}{lll}
\sum_{t} Y_{0}^{t_{1}} \mid=0, & \sum_{t} D_{0} \psi^{t} Y_{0}^{t 1} \mid=0, & Z_{0}^{5}\left|=b_{01}^{5} \sum_{t} \frac{Y_{0}^{t 1}}{D_{0} \psi^{t}}\right| \\
\sum_{t}\left(D_{0} \psi^{t}\right)^{2} Y_{0}^{t 1} \mid=0, & \left.\sum_{t}\left(D_{0} \psi^{t}\right)^{3} Y_{0}^{t 1}|=-| \eta\right|^{3} I^{4} ;
\end{array}
$$

on obtient les $Y_{0}^{t 1} \mid$ puis $Z_{0}^{5} \mid$ et tous les coefficients.

1b) $\left(b_{03}^{4}\right)^{2}+4 b_{11}^{4}|\eta|=0, C \equiv-D_{0} b_{03}^{4}-b_{12}^{4}|\eta|-\frac{2\left(b_{05}^{4} b_{11}^{5}+b_{15}^{4} b_{01}^{5}\right)}{b_{03}^{4}} \equiv 0$.

$$
\begin{aligned}
f & =\sum_{1 \leq t \leq 2} f_{1}^{t}+f_{2} \\
f_{1}^{t} & =e^{\psi^{t}}\left[Y_{0}^{t}+\cdots+Y_{k}^{t}+\cdots\right]
\end{aligned}
$$


$\left(D_{0} \psi^{t}\right)^{2}=\frac{b_{03}^{4}|\eta|}{2}, \psi^{t}$ est d'ordre $1 / 2 ; Y_{k}^{t}$ est d'ordre: $\frac{4-k}{2}$ :

$$
f_{2}=Z_{0}+\cdots+Z_{k}+\cdots
$$

$Z_{k}$ est d'ordre: $\frac{2-k}{2}$.

On reprend le calcul du cas 1a):

(5) $|\eta| Y_{5}^{4}+\cdots+\frac{3 D_{0} \psi}{|\eta|^{2}} D_{0}^{2} Y_{0}^{1}+W D_{0} Y_{0}^{1}-\frac{3\left(D_{0} \psi\right)^{3}}{|\eta|^{3}} D_{*} Y_{0}^{1}+W^{\prime} Y_{0}^{1}=0$

(6) à la $4^{\text {ème }}$ ligne, on obtient:

$$
-\frac{4\left(D_{0} \psi\right)^{2}}{|\eta|^{2}} D_{0}^{2} Y_{0}^{1}+W D_{0} Y_{0}^{1}+W^{\prime} Y_{0}^{1}=0
$$

le calcul en $Z$ reste le même.

On impose les conditions initiales.

$$
\begin{array}{ll}
\sum_{t} Y_{0}^{t} \mid=0 ; \quad & \sum_{t} Y_{1}^{t}=0 ; \quad \\
\sum_{t} Y_{3}^{t}\left|+Z_{1}\right|=0 ; & \sum_{t} Y_{4}^{t}\left|+Z_{2}\right|=I .
\end{array}
$$

On distingue les cas:

i) $b_{05}^{4} \neq 0, b_{01}^{5}=0$. Alors:

$$
\sum_{t} Y_{0}^{t 1}\left|=0 ; \quad \sum_{t} D_{0} \psi^{t} Y_{0}^{t 1}\right|=0
$$

d'où les

$$
Y_{0}^{t 1}\left|=0 ; \quad \sum_{t} D_{0} \psi^{t} D_{0} Y_{0}^{t 1}\right|=0 ;\left.\quad b_{03}^{4} \sum_{t} D_{0} Y_{0}^{t 1}|=-| \eta\right|^{2} I^{4}
$$

d'où les $D_{0} Y_{0}^{t 1} \mid$; on en déduit $Z_{0}^{1}$ en fonction de $I^{4}$ et de $I^{5}$.

ii) $b_{05}^{4}=0$. Alors:

$$
\sum_{t} Y_{0}^{t 1}\left|=0 ; \quad \sum_{t} D_{0} \psi^{t} Y_{0}^{t 1}\right|=0
$$

d'où $Y_{0}^{t 1} \mid=0$;

$\frac{4 b_{01}^{5}}{b_{03}^{4}} \sum_{t} D_{0} Y_{0}^{t 1}|+| \eta\left|Z_{0}^{5}\right|=0 ; \quad \sum_{t} D_{0} \psi^{t} D_{0} Y^{t 1}\left|=0 ; \quad b_{03}^{4} \sum_{t} D_{0} Y_{0}^{t 1}\right|=-|\eta|^{2} I^{4}$

d'où $D_{0} Y_{0}^{t 1} \mid$ et $Z_{0}^{5} \mid$. 
1c) $\left(b_{03}^{4}\right)^{2}+4 b_{11}^{4}|\eta|=0 ; C \neq 0$.

$$
\begin{aligned}
f & =\sum_{\substack{1 \leq t \leq 2 \\
1 \leq t^{\prime} \leq 2}} f_{1}^{t t^{\prime}}+f_{2} ; \\
f_{1}^{t t^{\prime}} & =e^{\psi_{1}^{t}+\psi_{2}^{t t^{\prime}}}\left[Y_{0}^{t t^{\prime}}+\cdots+Y_{k}^{t t^{\prime}}+\cdots\right]
\end{aligned}
$$

$\left(D_{0} \psi_{1}^{t}\right)^{2}=\frac{b_{03}^{4}|\eta|}{2} ; \psi_{1}^{t}$ est d'ordre $1 / 2 ;\left(D_{0} \psi_{2}^{t t^{\prime}}\right)^{2}=\frac{C D_{0} \psi_{1}^{t}}{2 b_{03}^{4}} ; \psi_{2}^{t t^{\prime}}$ est d'ordre $1 / 4 ; Y_{k}^{t t^{\prime}}$ est d'ordre $\frac{7-k}{4}$;

$$
f_{2}=Z_{0}+\cdots+Z_{k}+\cdots
$$

$Z_{k}$ est d'ordre $\frac{4-k}{4}$.

Les systèmes $Y$ et $Z$ sont ceux du $\$ 1.51$ c). Nous résumons fortement les calculs:

(0) $Y_{0}^{2}=Y_{0}^{3}=Y_{0}^{4} ; \quad$ (1) $\quad \ldots ; \quad$ (2) $Y_{0}^{5}=0$;

(8) (9) le choix de $D_{0} \psi_{1}$ laisse $Y_{0}^{1}$ libre.

(10) le choix de $D_{0} \psi_{2}$ laisse $Y_{0}^{1}$ libre.

(11) On obtient: $D_{0} Y_{0}^{1}+W Y_{0}^{1}=0$.

En $Z$ :

(0) $\quad Z_{0}^{2}=Z_{0}^{3}=Z_{0}^{4}=0$;

(1) (2) (3); (4) $\quad b_{05}^{4} Z_{0}^{5}=0, \quad D_{0} Z_{0}^{5}+b_{01}^{5} Z_{0}^{1}=0$;

on distingue:

i) $b_{05}^{4} \neq 0$. On obtient: $Z_{0}^{5}=0$ et à la $8^{\text {ème }}$ étape:

$$
D_{0} Z_{0}^{1}+W Z_{0}^{1}=0 \text {. }
$$

ii) $b_{05}^{4}=0 . \quad$ À la $8^{\text {ème }}$ étape on a:

$$
b_{11}^{4} Z_{0}^{1}+b_{15}^{4} Z_{0}^{5}=0
$$

d'où:

$$
D_{0} Z_{0}^{5}-\frac{b_{15}^{4} b_{01}^{5}}{b_{11}^{4}} Z_{0}^{5}=0
$$

On impose dans les cas i) et ii) les conditions initiales:

$$
\begin{aligned}
& \sum_{t} Y_{0}^{t}\left|=0 ; \quad \sum_{t} Y_{1}^{t}\right|=0 ; \\
& \sum_{t} Y_{2}^{t} \mid=0 \\
& \sum_{t} Y_{3}^{t}\left|+Z_{0}\right|=0 \\
& \sum_{t} Y_{4}^{t}\left|+Z_{1}\right|=0 \\
& \sum_{t} Y_{5}^{t}\left|+Z_{2}\right|=0 \\
& \sum_{t} Y_{6}^{t}\left|+Z_{5}\right|=0 \\
& \sum_{t} Y_{7}^{t}\left|+Z_{4}\right|=I
\end{aligned}
$$

On distingue les cas i) et ii) et on obtient les données initiales.

2) $b_{11}^{4}=0, b_{03}^{4} \neq 0$. Le 1 er segment de $N$ contient les points $0,(2,1)$. 
2a) $b_{15}^{4} b_{01}^{5}+b_{05}^{4} b_{11}^{5} \neq 0 . \quad$ Le $2^{\text {ème }}$ segment de $N$ contient les points $(2,1)$ et $(5,2)$; il est de pente $1 / 3$.

On cherche $f$ sous la forme:

$$
\begin{aligned}
f & =\sum_{1 \leq t \leq 2} f_{1}^{t}+\sum_{1 \leq t^{\prime} \leq 3} f_{2}^{t^{\prime}} ; \\
f_{1}^{t} & =e^{\psi_{t}}\left[Y_{0}^{t}+\cdots+Y_{k}^{t}+\cdots\right]
\end{aligned}
$$

$\left(D_{0} \psi^{t}\right)^{2}=b_{03}^{4}|\eta| ; \psi^{t}$ est d'ordre $1 / 2 ; Y_{k}^{t}$ est d'ordre $\frac{9-k}{6}$;

$$
\begin{gathered}
f_{2}^{t^{\prime}}=e^{\psi^{t^{\prime}}}\left[Z_{0}^{t^{\prime}}+\cdots+Z_{k}^{t^{\prime}}+\cdots\right] \\
b_{03}^{4}\left(D_{0} \chi^{t^{\prime}}\right)^{3}=b_{15}^{4} b_{01}^{5}+b_{05}^{4} b_{11}^{5}
\end{gathered}
$$

$\chi^{t^{\prime}}$ est d'ordre $1 / 3 ; Z_{k}^{t^{\prime}}$ est d'ordre $\frac{10-k}{6}$.

Les systèmes en $Y$ et $Z$ sont ceux du $\S 1.52 \mathrm{a}$ ).

Nous les résumons fortement:

$$
\text { (0) } Y_{0}^{2}=Y_{0}^{3}=Y_{0}^{4}=0 ; \quad \ldots ; \quad(3) \quad Y_{0}^{5}=0 ; \quad \ldots \text {; }
$$

(12) le choix de $D_{0} \psi_{1}$ laisse $Y_{0}^{1}$ libre;

$$
\text { (15) } D_{0} Y_{0}^{1}+W Y_{0}^{1}=0
$$

Pour les $Z$ :
(0) $Z_{0}^{2}=Z_{0}^{3}=Z_{0}^{4}=0$;
(4) $Z_{0}^{5}=0 ; \quad \ldots$;

(14) le choix de $D_{0} \chi$ laisse $Z_{0}^{1}$ libre: ...;

$$
\text { (16) } D_{0} Z_{0}^{1}+W Z_{0}^{1}=0 \text {. }
$$

On impose les conditions initiales.

$$
\begin{array}{ll}
\sum_{t^{\prime}} Z_{0}^{t^{\prime}} \mid=0 ; & \sum_{t} Y_{0}^{t}\left|+\sum_{t^{\prime}} Z_{1}^{t^{\prime}}\right|=0 ; \quad \ldots ; \\
\sum_{t} Y_{8}^{t}\left|+\sum_{t} Z_{9}^{t^{\prime}}\right|=0 ; & \sum_{t} Y_{9}^{t}\left|+\sum_{t^{\prime}} Z_{10}^{t^{\prime}}\right|=I
\end{array}
$$

En distinguant selon que $b_{05}^{4} \neq 0$ ou $b_{05}^{4}=0$, on obtient les données initiales $Y_{0}^{t 1}\left|, Z_{0}^{t^{\prime} 1}\right|$ et tous les coefficients. 
2b) $\quad b_{15}^{4} b_{01}^{5}+b_{05}^{4} b_{11}^{5}=0 . \quad$ Le $2^{\text {ème }}$ segment est horizontal.

$$
\begin{aligned}
f & =\sum_{1 \leq t \leq 2} f_{1}^{t}+f_{2} \\
f_{1}^{t} & =e^{\psi^{t}}\left[Y_{0}^{t}+\cdots+Y_{k}^{t}+\cdots\right]
\end{aligned}
$$

$\left(D_{0} \psi^{t}\right)^{2}=b_{03}^{4}|\eta| ; \psi^{t}$ est d'ordre $1 / 2 ; Y_{k}^{t}$ est d'ordre $\frac{3-k}{2} ;$

$$
f_{2}=Z_{0}+\cdots+Z_{k}
$$

$Z_{k}$ est d'ordre $\frac{4-k}{2}$.

Les systèmes en $Y$ et $Z$ sont ceux du cas 1a). On obtient:

$$
Y_{0}^{2}=Y_{0}^{3}=Y_{0}^{4}=Y_{0}^{5}=0
$$

à la $4^{\text {ème }}$ étape, $Y_{0}^{1}$ reste libre par le choix de $D_{0} \psi$; à la $5^{\text {ème }}$ étape, on a:

$$
D_{0} Y_{0}^{1}+W Y_{0}^{1}=0
$$

On a ainsi:

$$
Z_{0}^{2}=Z_{0}^{3}=Z_{0}^{4}=0
$$

i) Si $b_{05}^{4} \neq 0 Z_{0}^{5}=0$ et:

$$
D_{0}^{3} Z_{0}^{1}+W D_{0}^{2} Z_{0}^{1}+W^{\prime} D_{0} Z_{0}^{1}+W^{\prime \prime} Z_{0}^{1}=0 .
$$

ii) Si $b_{05}^{4}=0$, on a: $b_{15}^{4} b_{01}^{5}=0$, on doit distinguer:

ii') $b_{15}^{4} \neq 0$, où l'on obtient: $Z_{0}^{5}=0$,

$$
D_{0}^{3} Z_{0}^{1}+W D_{0}^{2} Z_{0}^{1}+W^{\prime} D_{0} Z_{0}^{1}+W^{\prime \prime} Z_{0}^{1}=0 .
$$

$\left.\mathbf{i i}^{\prime \prime}\right) b_{15}^{4}=0, \quad$ où l'on obtient:

$$
D_{0} Z_{0}^{5}+b_{01}^{5} Z_{0}^{1}=0 ; \quad D_{0}^{2} Z_{0}^{1}+W D_{0} Z_{0}^{1}+W^{\prime} Z_{0}^{1}=0 .
$$

On impose les conditions initiales:

$$
Z_{0}\left|=0 ; \quad \sum_{t} Y_{0}^{t}\right|+Z_{1}=0 ; \ldots ; \quad \sum_{t} Y_{2}^{t}\left|+Z_{3}\right|=0 ; \quad \sum_{t} Y_{3}^{t}\left|+Z_{4}\right|=I ; \ldots ;
$$

dans les différents cas, on obtient aisément les données initiales. 


\subsection{Cas $(\mathrm{LG})_{5 / 2}$.}

$$
\begin{aligned}
f & =\sum_{1 \leq t \leq 5} f_{1}^{t} ; \\
f_{1}^{t} & =e^{\psi_{1}+\psi_{2}}\left[Y_{0}^{t}+\cdots+Y_{k}^{t}+\cdots\right] ;
\end{aligned}
$$

$\left(D_{0} \psi_{1}^{t}\right)^{5}=-|\eta|^{3}\left(b_{05}^{4} b_{11}^{5}+b_{15}^{4} b_{01}^{5}\right) ; \psi_{1}^{t}$ est d'ordre $2 / 5$ :

$D_{0} \psi_{2}^{t}=\frac{-\left(D_{0} \psi_{1}^{t}\right)^{3} b_{12}^{4}}{3\left(b_{05}^{t} b_{11}^{5}+b_{15}^{t} b_{01}^{5}\right)|\eta|} ; \psi_{2}^{t}$ est d'ordre $1 / 5 ; Y_{k}^{t}$ est d'ordre: $\frac{9-k}{5}$.

Les systèmes en $Y^{5}$ et $Z$ sont ceux du $\S 1.6$.

On obtient, en résumant fortement:

$$
\text { (0) } Y_{0}^{2}=Y_{0}^{3}=Y_{0}^{4}=0 ; \quad \ldots ; \quad(3) \quad Y_{0}^{5}=0 ; \quad \ldots \text {; }
$$

(12) le choix de $D_{0} \psi_{1}$ laisse $Y_{0}^{1}$ libre; (13) le choix de $D_{0} \psi_{2}$ laisse $Y_{0}^{1}$ libre; (14) donne: $D_{0} Y_{0}^{1}+W Y_{0}^{1}=0$.

On impose les données initiales:

$$
\sum_{t} Y_{0}^{t}\left|=0=\cdots=\sum_{t} Y_{8}^{t}\right|=0 ; \quad \sum_{t} Y_{9}^{t} \mid=I ; \quad \cdots ;
$$

On distingue les cas i) $b_{05}^{4} \neq 0$ et ii) $b_{05}^{4}=0$ et on obtient aisément les $Y_{0}^{t 1} \mid$, d'où les $Y_{0}^{t}$ et tous les coefficients.

\subsection{Cas $(\mathbf{L G})_{3}$.}

$$
\begin{aligned}
f & =\sum_{1 \leq t \leq 3} f_{1}^{t}+f_{2} ; \\
f_{1}^{t} & =e^{\psi_{t}}\left[Y_{0}^{t}+\cdots+Y_{k}^{t}+\cdots\right]
\end{aligned}
$$

$\left(D_{0} \psi^{t}\right)^{3}=-b_{12}^{4}|\eta|^{2} ; \psi^{t}$ est d'ordre $1 / 3 ; Y_{k}^{t}$ est d'ordre: $\frac{6-k}{3}$;

$$
f_{2}=Z_{0}+\cdots Z_{k}+\cdots
$$

$Z_{k}$ est d'ordre: $\frac{6-k}{3}$.

On a:

$$
\text { (0) } Y_{0}^{2}=Y_{0}^{3}=Y_{0}^{4}=0 ; \ldots ; \quad \text { (3) } Y_{0}^{5}=0 ; \quad \ldots ;
$$

(8) le choix de $D_{0} \psi$ laisse $Y_{0}^{1}$ libre; (9) $D_{0} Y_{0}^{1}+W Y_{0}^{1}=0$.
(0) $Z_{0}^{2}=Z_{0}^{3}=Z_{0}^{4}=0$;
(3) $b_{05}^{4} Z_{0}^{5}=0$
$D_{0} Z_{0}^{5}+b_{01}^{5} Z_{0}^{1}=0$.

On distingue deux sous cas

i) $b_{05}^{4} \neq 0 . \quad Z_{0}^{5}=0$; à l'étape (9), on obtient:

$$
D_{0}^{2} Z_{0}^{1}+W D_{0} Z_{0}^{1}+W^{\prime} Z_{0}^{1}=0 .
$$

ii) $b_{05}^{4}=0 . \quad$ On distingue encore: 
ii) $\quad b_{15}^{4} \neq 0 . \quad Z_{0}^{5}=0$, à l'étape $(9)$, on a: $D_{0}^{2} Z_{0}^{1}+W D_{0} Z_{0}^{1}+W^{\prime} Z_{0}^{1}=0$.

ii) ${ }^{\prime \prime} \quad b_{15}^{4}=0 . \quad(9)$ donne $D_{0} Z_{0}^{1}+W Z_{0}^{1}+W^{\prime} Z_{0}^{5}=0$.

On impose:

$$
\sum_{t} Y_{0}^{t}\left|+Z_{0}\right|=0 \ldots=\sum_{t} Y_{5}^{t}\left|+Z_{5}\right| ; \quad \sum_{t} Y_{6}^{t}\left|+Z_{6}\right|=I ; \ldots
$$

en distinguant les différents cas, on obtient aisément les données initiales et tous les coefficients.

\subsection{Cas (LG) $)_{4}$.}

$$
\begin{aligned}
f & =\sum_{1 \leq t \leq 4} f_{1}^{t}+f_{2} \\
f_{1}^{t} & =e^{\psi^{t}}\left[Y_{0}^{t}+\cdots+Y_{k}^{t}+\cdots\right]
\end{aligned}
$$

$\left(D_{0} \psi^{t}\right)^{4}=b_{21}^{4}|\eta|^{3} ; \psi^{t}$ est d'ordre $1 / 4 ; Y_{k}^{t}$ est d'ordre: $\frac{9-k}{4}$;

$$
f_{2}=Z_{0}+\cdots+Z_{k}+\cdots
$$

$Z_{k}$ est d'ordre $\frac{8-k}{4}$.

On a:

$$
\text { (0) } Y_{0}^{2}=Y_{0}^{3}=Y_{0}^{4}=0 ; \quad \ldots ; \quad(3) \quad Y_{0}^{5}=0 ; \quad \ldots ;
$$

(12) laisse $Y_{0}^{1}$ libre par le choix de $D_{0} \psi ;(13)$ donne: $D_{0} Y_{0}^{1}+W Y_{0}^{1}=0$.
(0) $Z_{0}^{2}=Z_{0}^{3}=Z_{0}^{4}=0$
(4) $b_{05}^{4} Z_{0}^{5}=0$
$D_{0} Z_{05}^{5}+b_{01}^{5} Z_{0}^{1}=0$

On distingue les sous-cas:

i) $b_{05}^{4} \neq 0 . \quad Z_{0}^{5}=0$, à l'étape (12), on a: $D_{0} Z_{0}^{1}+W Z_{0}^{1}=0$.

ii) ${ }^{\prime} \quad b_{05}^{4}=0, b_{15}^{4} \neq 0 . \quad$ On a: $Z_{0}^{5}=0, D_{0} Z_{0}^{1}+W Z_{0}^{1}=0$;

ii) ${ }^{\prime \prime} \quad b_{05}^{4}=0=b_{15}^{4} \neq 0$. On a: $b_{21}^{4} Z_{0}^{1}+b_{25}^{4} Z_{0}^{5}=0$, d'où: $D_{0} Z_{0}^{5}+W Z_{0}^{5}=0$.

On impose:

$$
\sum_{t} Y_{0}^{t}\left|=0 ; \quad \sum_{t} Y_{1}^{t}\right|+Z_{0}\left|=0 ; \quad \ldots ; \quad \sum_{t} Y_{9}^{t}\right|+Z_{8} \mid=I ; \quad \ldots ;
$$

on en déduit les données initiales des différents cas et tous les coefficients. 
2.8. Cas $(\mathbf{L G})_{5}$.

$\psi^{t}$ est d'ordre $1 / 5 ; Y_{k}^{t}$ est d'ordre $\frac{12-k}{5}$.

$$
\begin{aligned}
f & =\sum_{1 \leq t \leq 5} f_{1}^{t} ; \\
f_{1}^{t} & =e^{\psi^{t}}\left[Y_{0}^{t}+\cdots+Y_{k}^{t}+\cdots\right] ; \\
\left(D_{0} \psi^{t}\right)^{5} & =-|\eta|^{3}\left(b_{15}^{4} b_{11}^{5}+b_{25}^{4} b_{01}^{5}+b_{01}^{4} b_{21}^{5}\right) ;
\end{aligned}
$$

On a:

$$
\text { (0) } Y_{0}^{2}=Y_{0}^{3}=Y_{0}^{4}=0 ; \quad \ldots ; \quad \text { (4) } Y_{0}^{5}=0 ; \quad \ldots \text {; }
$$

(16) le choix de $D_{0} \psi$ laisse $Y_{0}^{1}$ libre:

$$
\text { (17) } D_{0} Y_{0}^{1}+W Y_{0}^{1}=0 \text {. }
$$

On impose les conditions initiales:

$$
\sum_{t} Y_{0}^{t}\left|=0 ; \quad \sum_{t} Y_{1}^{t}\right|=0 ; \quad \ldots ; \quad \sum_{t} Y_{12}^{t} \mid=I ; \quad \ldots ;
$$

Dans les différents cas, on obtient: $Y_{0}^{t 1} \mid$ et tous les coefficients.

\subsection{Cas L.}

$$
f=Y_{0}+\cdots+Y_{k}+\cdots
$$

$Y_{k}$ est d'ordre $3-k$.

On a:

(0) $Y_{0}^{2}=Y_{0}^{3}=Y_{0}^{4}=0$;

(1) $|\eta| Y_{1}^{2}+D_{0} Y_{0}^{1}=0 b_{05}^{4} Y_{0}^{5}=0, \quad D_{0} Y_{0}^{5}+b_{01}^{5} Y_{0}^{1}=0$.

On distingue deux cas:

i) $b_{05}^{4} \neq 0, b_{01}^{5}=0, Y_{0}^{5}=0$.

(2) $|\eta| Y_{2}^{3}-\frac{D_{0}^{2} Y_{0}^{1}}{|\eta|^{2}}=0 ; \quad \ldots$

(3) $|\eta| Y_{3}^{4}+\frac{D_{0}^{3} Y_{0}^{1}}{|\eta|^{3}}=0 ; \quad \ldots$

(4) $-\frac{D_{0}^{4} Y_{0}^{1}}{|\eta|^{3}}+b_{05}^{4} Y_{3}^{5}+\frac{b_{13}^{4} D_{0}^{2} Y_{0}^{1}}{|\eta|^{2}}-\frac{b_{22}^{4} D_{0} Y_{0}^{1}}{|\eta|}+b_{31}^{4} Y_{0}^{1}=0$

$$
D_{0} Y_{3}^{5}+b_{31}^{5} Y_{0}^{1}=0
$$

d'où:

$$
D_{0}^{5} Y_{0}^{1}+W D_{0}^{4} Y_{0}^{1}+W^{\prime} D_{0}^{3} Y_{0}^{1}+W^{\prime \prime} D_{0}^{2} Y_{0}^{1}+W^{\prime \prime \prime} D_{0} Y_{0}^{1}+W^{I V} Y_{0}^{1}=0 .
$$

On impose les conditions initiales:

$$
\sum_{t} Y_{0}^{t}\left|=0=\sum_{t} Y_{1}^{t}\right|=\sum_{t} Y_{2}^{t}\left|; \quad \sum_{t} Y_{3}^{t}\right|=I ; \quad \cdots
$$


d'où l'on déduit aisément les données initiales et tous les coefficients.

ii) $)^{\prime} \quad b_{05}^{4}=0, b_{15}^{4} \neq 0, b_{01}^{5}=b_{11}^{5}=0$.

(2) donne $Y_{0}^{5}=0$;

(3) $D_{0} Y_{2}^{5}+b_{21}^{5} Y_{0}^{1}=0, \quad Y_{1}^{5}=0$;

(4) $-\frac{D_{0}^{4} Y_{0}^{1}}{|\eta|^{3}}+b_{13}^{4} \frac{D_{0}^{2} Y_{0}^{1}}{|\eta|^{2}}+b_{15}^{4} Y_{2}^{5}-\frac{2 b_{22}^{4} D_{0} Y_{0}^{1}}{|\eta|}+b_{31}^{4} Y_{0}^{1}=0$.

Il suffit de déterminer les données initiales $Y_{0}^{1}\left|, D_{0} Y_{0}^{1}\right|, D_{0}^{2} Y_{0}^{1}\left|, D_{0}^{3} Y_{0}^{1}\right|, D_{0}^{4} Y_{0}^{1} \mid$.

On impose les mêmes conditions que précédemment et on obtient les données initiales.

ii) ${ }^{\prime} \quad b_{05}^{4}=b_{15}^{4}=0 . \quad$ On doit encore distinguer $b_{01}^{5} \neq 0$ ou $b_{01}^{5}=0$.

Si $b_{01}^{5} \neq 0$, on a:

$$
\text { (4) }-\frac{D_{0}^{4} Y_{0}^{1}}{|\eta|^{3}}+b_{13}^{4} \frac{D_{0}^{2} Y_{0}^{1}}{|\eta|^{2}}-b_{22}^{4} \frac{D_{0} Y_{0}^{1}}{|\eta|}=0 \text {. }
$$

On impose les mêmes conditions initiales et on obtient les données et tous les coefficients.

Si $b_{01}^{5}=0$

$$
\begin{aligned}
& \text { (2) } D_{0} Y_{1}^{5}+b_{11}^{5} Y_{0}^{1}=0 ; \quad \text { (3) } b_{25}^{4} Y_{0}^{5}=0 ; \\
& \text { (4) }-\frac{D_{0}^{4} Y_{0}^{1}}{|\eta|^{3}}+b_{13}^{4} \frac{D_{0}^{2} Y_{0}^{1}}{|\eta|^{2}}-b_{22}^{4} \frac{D_{0} Y_{0}^{1}}{|\eta|}+b_{25}^{4} Y_{1}^{5}=0 .
\end{aligned}
$$

On distingue encore $b_{25}^{4} \neq 0$ ou $b_{25}^{4}=0$; dans le 1er cas: $Y_{0}^{5}=0$ et on a une équation de propagation d'ordre 5 en $Y_{0}^{1}$; dans le $2^{\text {ème }}$ cas; on a une équation de propagation d'ordre 4 en $Y_{0}^{1}$ et

$$
\text { (1) } D_{0} Y_{0}^{5}=0
$$

On impose les mêmes conditions initiales et on obtient tous les coefficients.

\section{§3. Étude du type $(3,2)$}

$$
h=\left(\begin{array}{ccccc}
D_{0} & \left|D^{\prime}\right| & 0 & 0 & 0 \\
0 & D_{0} & \left|D^{\prime}\right| & 0 & 0 \\
b_{1}^{3} & b_{2}^{3} & D_{0} & b_{4}^{3} & b_{5}^{3} \\
b_{1}^{4} & 0 & 0 & D_{0} & \left|D^{\prime}\right| \\
b_{1}^{5} & 0 & 0 & b_{4}^{5} & D_{0}
\end{array}\right)
$$


Les conditions $(\mathrm{LG})_{d}$ s'écrivent

$(\mathrm{LG})_{3 / 2}: b_{01}^{3} \not \equiv 0$;

$(\mathrm{LG})_{5 / 3}: b_{01}^{3} \equiv 0, b_{04}^{3} b_{01}^{5} \not \equiv 0$;

$(\mathrm{LG})_{2}: b_{01}^{3} \equiv 0, b_{04}^{3} b_{01}^{5} \equiv 0$,

$\left(b_{04}^{3}\right.$ ou $b_{01}^{5} \not \equiv 0, b_{04}^{3} b_{01}^{4}+b_{05}^{3} b_{01}^{5}-b_{02}^{3} b_{04}^{5} \not \equiv 0$ ou $\left.b_{02}^{3}+b_{04}^{5} \not \equiv 0\right)$;

ou

$\left(b_{04}^{3} \equiv b_{01}^{5} \equiv 0, b_{02}^{3} \not \equiv 0\right.$ ou $\left.b_{04}^{5} \not \equiv 0\right)$;

$(\mathrm{LG})_{5 / 2}$ : on définit:

si $b_{04}^{3} \not \equiv 0, C^{\prime} \equiv b_{05}^{3} b_{04}^{5} b_{01}^{4}+b_{02}^{3} b_{11}^{3}\left|\xi^{\prime}\right|+b_{04}^{3} b_{11}^{5}\left|\xi^{\prime}\right|-b_{04}^{3} D_{0} b_{01}^{4} ;$

si $b_{01}^{5} \not \equiv 0, C^{\prime \prime} \equiv b_{05}^{3} b_{04}^{5} b_{01}^{4}+b_{02}^{3} b_{11}^{3}\left|\xi^{\prime}\right|+b_{01}^{5} b_{14}^{3}\left|\xi^{\prime}\right|-b_{02}^{3} D_{0} b_{02}^{3}-b_{05}^{3} D_{0} b_{01}^{5}$;

on définit $C=C^{\prime}$ ou $C^{\prime \prime}$ selon le cas.

La condition $(\mathrm{LG})_{5 / 2}$ est:

$\left(b_{04}^{3} \not \equiv 0, b_{01}^{3} \equiv b_{01}^{5} \equiv b_{02}^{3}+b_{04}^{5} \equiv\left(b_{02}^{3}\right)^{2}+b_{04}^{3} b_{01}^{4} \equiv 0, C^{\prime} \neq 0\right)$;

ou

$\left(b_{01}^{5} \not \equiv 0, b_{01}^{3} \equiv b_{04}^{3} \equiv b_{02}^{3}+b_{04}^{5} \equiv\left(b_{02}^{3}\right)^{2}+b_{05}^{3} b_{01}^{5} \equiv 0, C^{\prime \prime} \neq 0\right) ;$

$(\mathrm{LG})_{3}$ : on définit:

si $b_{04}^{3} \not \equiv 0, C_{3}^{\prime} \equiv b_{11}^{3}\left|\xi^{\prime}\right|-2 b_{04}^{3} D_{0}\left(\frac{b_{02}^{3}}{b_{04}^{3}}\right)$;

si $b_{01}^{5} \not \equiv 0, C_{3}^{\prime \prime} \equiv b_{11}^{3}\left|\xi^{\prime}\right|+D_{0} b_{02}^{3}+2 b_{01}^{5} D_{0}\left(\frac{b_{02}^{3}}{b_{01}^{5}}\right)$;

on définit $C_{3}=C_{3}^{\prime}$ ou $C_{3}^{\prime \prime}$ selon le cas.

La condition $(\mathrm{LG})_{3}$ est

$\left(b_{04}^{3} \not \equiv 0, b_{01}^{3} \equiv b_{01}^{5} \equiv b_{02}^{3}+b_{04}^{5} \equiv\left(b_{02}^{3}\right)^{2}+b_{04}^{3} b_{01}^{4} \equiv 0, C^{\prime} \equiv 0, C_{3}^{\prime} \not \equiv 0\right)$;

ou

$\left(b_{01}^{5} \not \equiv 0, b_{01}^{3} \equiv b_{04}^{3} \equiv b_{02}^{3}+b_{04}^{5} \equiv\left(b_{02}^{3}\right)^{2}+b_{05}^{3} b_{01}^{5} \equiv 0, C^{\prime \prime} \equiv 0, C_{3}^{\prime \prime} \not \equiv 0\right)$;

ou

$\left(b_{01}^{3} \equiv b_{04}^{3} \equiv b_{01}^{5} \equiv b_{02}^{3} \equiv b_{04}^{5} \equiv 0, b_{11}^{3} \not \equiv 0\right)$;

$(\mathrm{LG})_{4}$ : on définit:

si $b_{04}^{3} \not \equiv 0, b_{04}^{3} C_{4}^{\prime} \equiv\left(b_{05}^{3}\right)^{2} b_{04}^{5} b_{01}^{4}-b_{05}^{3} b_{04}^{5} b_{11}^{3}\left|\xi^{\prime}\right|+b_{04}^{3} b_{04}^{5} b_{12}^{3}\left|\xi^{\prime}\right|-b_{04}^{3} b_{01}^{4} b_{14}^{3}\left|\xi^{\prime}\right|$ $-\left(b_{04}^{3}\right)^{2} b_{11}^{4}\left|\xi^{\prime}\right|+b_{04}^{3} b_{02}^{3} b_{14}^{5}\left|\xi^{\prime}\right|+b_{04}^{3} b_{05}^{3} b_{04}^{5} D_{0}\left(\frac{b_{02}^{3}}{b_{04}^{3}}\right)$ 


$$
\begin{aligned}
& -2\left(b_{04}^{3}\right)^{2} D_{0}\left(\frac{b_{05}^{3} b_{01}^{4}}{b_{04}^{3}}\right)+2\left(b_{04}^{3}\right)^{2} D_{0}\left(\frac{b_{11}^{3}}{b_{04}^{3}}\right)\left|\xi^{\prime}\right|-\left(b_{04}^{3}\right)^{2} D_{0}^{2}\left(\frac{b_{02}^{3}}{b_{04}^{3}}\right) \\
& +\left(b_{04}^{3}\right)^{2} D_{*} b_{01}^{4}+b_{04}^{3}\left[b_{02}^{3}\left(D b_{04}^{3}\right)_{*}-b_{04}^{3}\left(D b_{02}^{3}\right)_{*}\right]\left(\frac{b_{02}^{3}}{b_{04}^{3}}\right)=0
\end{aligned}
$$

si $b_{01}^{5} \not \equiv 0, b_{01}^{5} C_{4}^{\prime \prime} \equiv b_{05}^{3} b_{04}^{5}\left(b_{01}^{4}\right)^{2}-b_{04}^{5} b_{01}^{4} b_{11}^{3}\left|\xi^{\prime}\right|+b_{01}^{5} b_{04}^{5} b_{12}^{3}\left|\xi^{\prime}\right|-\left(b_{01}^{5}\right)^{2} b_{15}^{3}\left|\xi^{\prime}\right|$

$$
\begin{aligned}
& -b_{02}^{3} b_{04}^{5} b_{11}^{5}\left|\xi^{\prime}\right|+b_{01}^{5} b_{02}^{3} b_{14}^{5}\left|\xi^{\prime}\right|-3\left(b_{01}^{5}\right)^{2} D_{0}^{2}\left(\frac{b_{04}^{5}}{b_{01}^{5}}\right) \\
& +b_{02}^{3} b_{04}^{5} D_{0} b_{01}^{4}+b_{02}^{3} b_{01}^{4} b_{01}^{5} D_{0}\left(\frac{b_{04}^{5}}{b_{01}^{5}}\right)+b_{02}^{3}\left(b_{01}^{5}\right)^{2} D_{0}\left(\frac{b_{04}^{5} b_{01}^{4}}{\left(b_{01}^{5}\right)^{2}}\right) \\
& -b_{02}^{3}\left(b_{01}^{5}\right)^{2} D_{*}\left(\frac{b_{04}^{5}}{b_{01}^{5}}\right)\left|\xi^{\prime}\right|+b_{01}^{5}\left[b_{02}^{3}\left(D b_{01}^{5}\right)_{*}-b_{01}^{5}\left(D b_{02}^{3}\right)_{*}\right]\left(\frac{b_{02}^{3}}{b_{01}^{5}}\right)\left|\xi^{\prime}\right|=0
\end{aligned}
$$

on définit $C_{4}=C_{4}^{\prime}$ ou $C_{4}^{\prime \prime}$ selon le cas.

La condition (LG) $)_{4}$ est

$$
\left(b_{04}^{3} \not \equiv 0, b_{01}^{3} \equiv b_{01}^{5} \equiv b_{02}^{3}+b_{04}^{5} \equiv\left(b_{02}^{3}\right)^{2}+b_{04}^{3} b_{01}^{4} \equiv 0, C^{\prime} \equiv C_{3}^{\prime} \equiv 0, C_{4}^{\prime} \not \equiv 0\right) \text {; }
$$
ou

$\left(b_{01}^{5} \not \equiv 0, b_{01}^{3} \equiv b_{04}^{3} \equiv b_{02}^{3}+b_{04}^{5} \equiv\left(b_{02}^{3}\right)^{2}+b_{05}^{3} b_{01}^{5} \equiv 0, C^{\prime \prime} \equiv C_{3}^{\prime \prime} \equiv 0, C_{4}^{\prime \prime} \not \equiv 0\right)$;

ou

$$
\begin{aligned}
& \left(b_{01}^{3} \equiv b_{04}^{3} \equiv b_{01}^{5} \equiv b_{02}^{3} \equiv b_{04}^{5} \equiv 0, b_{11}^{3} \not \equiv 0\right) ; \text { ou } \\
& \left(b_{04}^{3} \equiv b_{01}^{5} \equiv b_{02}^{3} \equiv b_{04}^{5} \equiv b_{11}^{3} \equiv 0, b_{14}^{3} b_{01}^{4}+b_{05}^{3} b_{11}^{5} \not \equiv 0\right) ;
\end{aligned}
$$

$(\mathrm{LG})_{5}$ : si $b_{04}^{3} \not \equiv 0, C_{5}^{\prime} \equiv b_{15}^{3} b_{04}^{5} b_{01}^{4}\left|\xi^{\prime}\right|+b_{05}^{3} b_{14}^{5} b_{01}^{4}\left|\xi^{\prime}\right|+b_{05}^{3} b_{04}^{5} b_{11}^{4}\left|\xi^{\prime}\right|-b_{11}^{3} b_{14}^{5}\left|\xi^{\prime}\right|^{2}$

$$
\begin{aligned}
& +b_{14}^{3} b_{11}^{5}\left|\xi^{\prime}\right|^{2}-b_{21}^{3} b_{04}^{5}\left|\xi^{\prime}\right|^{2}+b_{04}^{3} b_{21}^{5}\left|\xi^{\prime}\right|^{2}-\left|\xi^{\prime}\right| b_{02}^{3}\left(D b_{05}^{3}\right)_{*} b_{01}^{4} \\
& +b_{05}^{3} b_{04}^{5} D_{0}\left(\frac{b_{05}^{3} b_{01}^{4}-b_{11}^{3}\left|\xi^{\prime}\right|}{b_{04}^{3}}\right)-b_{04}^{3} D_{0}^{2}\left(\frac{b_{05}^{3} b_{01}^{4}-b_{11}^{3}\left|\xi^{\prime}\right|}{b_{04}^{3}}\right) \\
& -b_{05}^{3} b_{04}^{5} D_{*} b_{01}^{4}+b_{04}^{3} D_{0} D_{*} b_{01}^{4}-b_{04}^{3} D_{0} b_{11}^{4}\left|\xi^{\prime}\right|-b_{14}^{3} D_{0} b_{01}^{4}\left|\xi^{\prime}\right| \\
& +\left[b_{04}^{3}\left(D b_{02}^{3}\right)_{*}-b_{02}^{3}\left(D b_{04}^{3}\right)_{*}\right]\left(\frac{b_{11}^{3}\left|\xi^{\prime}\right|-b_{05}^{3} b_{01}^{4}}{b_{04}^{3}}\right)\left|\xi^{\prime}\right|
\end{aligned}
$$

si $b_{01}^{5} \not \equiv 0, C_{5}^{\prime \prime} \equiv b_{15}^{3} b_{04}^{5} b_{01}^{4}\left|\xi^{\prime}\right|+b_{05}^{3} b_{14}^{5} b_{01}^{4}\left|\xi^{\prime}\right|+b_{05}^{3} b_{04}^{5} b_{11}^{4}\left|\xi^{\prime}\right|-b_{11}^{3} b_{14}^{5}\left|\xi^{\prime}\right|^{2}$

$$
\begin{aligned}
& +b_{14}^{3} b_{11}^{5}\left|\xi^{\prime}\right|^{2}-b_{21}^{3} b_{04}^{5}\left|\xi^{\prime}\right|^{2}+b_{24}^{3} b_{01}^{5}\left|\xi^{\prime}\right|^{2}+\frac{b_{05}^{3} b_{01}^{4}-b_{11}^{3}\left|\xi^{\prime}\right|}{b_{01}^{5}} D_{0}\left(\frac{b_{04}^{5} b_{01}^{4}}{b_{01}^{5}}\right) \\
& +b_{02}^{3} D^{2}\left(\frac{b_{04}^{5} b_{01}^{4}}{b_{01}^{5}}\right)-b_{02}^{3} D_{0}\left(\frac{b_{04}^{5} b_{01}^{4}}{b_{01}^{4}}\right)\left(\frac{D_{0} b_{01}^{5}}{b_{01}^{5}}\right)-b_{01}^{5} D_{0}^{3}\left(\frac{b_{04}^{5}}{b_{01}^{5}}\right) \\
& -b_{05}^{3} b_{01}^{5} D_{0}\left(\frac{b_{11}^{5}}{b_{01}^{5}}\right)\left|\xi^{\prime}\right|+b_{02}^{3} b_{01}^{5} D_{0}\left(\frac{b_{14}^{5}}{b_{01}^{5}}\right)\left|\xi^{\prime}\right|+b_{12}^{3} b_{01}^{5} D_{0}\left(\frac{b_{04}^{5}}{b_{01}^{5}}\right)\left|\xi^{\prime}\right| \\
& -b_{05}^{3} b_{01}^{5} D_{*}\left(\frac{b_{04}^{5} b_{01}^{4}}{b_{01}^{5}}\right)-b_{02}^{3} b_{01}^{5} D_{0} D_{*}\left(\frac{b_{04}^{5}}{b_{01}^{5}}\right)+b_{11}^{3} D_{*}\left(\frac{b_{04}^{5}}{b_{01}^{5}}\right) b_{01}^{5}\left|\xi^{\prime}\right| \\
& +\left(D\left(b_{05}^{3} b_{01}^{4}\right)\right)_{*}\left(\frac{b_{04}^{5}}{b_{01}^{5}}\right)\left|\xi^{\prime}\right|+\left(\frac{b_{04}^{5}}{b_{01}^{5}}\right)\left(D b_{05}^{3}\right)_{*} b_{01}^{4}\left|\xi^{\prime}\right| \\
& +\left(\frac{b_{11}^{3}\left|\xi^{\prime}\right|-b_{05}^{3} b_{01}^{4}}{b_{01}^{5}}\right)\left(D b_{01}^{5}\right)_{*}\left(\frac{b_{04}^{5}}{b_{01}^{5}}\right)\left|\xi^{\prime}\right|-\left(D b_{11}^{3}\right)_{*}\left(\frac{b_{04}^{5}}{b_{01}^{5}}\right)\left|\xi^{\prime}\right|^{2} \\
& -b_{02}^{3} D_{0}\left[\frac{1}{b_{01}^{5}}\left(D b_{01}^{5}\right)_{*}\left(\frac{b_{04}^{5}}{b_{01}^{5}}\right)\right]\left|\xi^{\prime}\right|+\left(D b_{02}^{3}\right)_{*}\left[D_{0}\left(\frac{b_{04}^{5}}{b_{01}^{5}}\right)\right]\left|\xi^{\prime}\right|
\end{aligned}
$$

on définit $C_{5}=C_{5}^{\prime}$ ou $C_{5}^{\prime \prime}$ selon le cas. 
La condition (LG) $)_{5}$ est

$\left(b_{04}^{3} \not \equiv 0, b_{01}^{3} \equiv b_{01}^{5} \equiv b_{02}^{3}+b_{04}^{5} \equiv\left(b_{02}^{3}\right)^{2}+b_{04}^{3} b_{01}^{4} \equiv 0, C^{\prime} \equiv C_{3}^{\prime} \equiv C_{4}^{\prime} \equiv 0\right.$,

$\left.C_{5}^{\prime} \not \equiv 0\right)$;

ou

$\left(b_{01}^{5} \not \equiv 0, b_{01}^{3} \equiv b_{04}^{3} \equiv b_{02}^{3}+b_{04}^{5} \equiv\left(b_{02}^{3}\right)^{2}+b_{05}^{3} b_{01}^{5} \equiv 0, C^{\prime \prime} \equiv C_{3}^{\prime \prime} \equiv C_{4}^{\prime \prime} \equiv 0\right.$,

$\left.C_{5}^{\prime \prime} \not \equiv 0\right)$

ou

$\left(b_{01}^{3} \equiv b_{04}^{3} \equiv b_{02}^{3} \equiv b_{01}^{5}=b_{04}^{5} \equiv b_{11}^{3} \equiv b_{14}^{3} b_{01}^{4}+b_{05}^{3} b_{11}^{5} \equiv 0\right.$,

$\left.b_{05}^{3} b_{14}^{5} b_{01}^{4}+b_{14}^{3} b_{11}^{5}\left|\xi^{\prime}\right|+b_{14}^{3} D_{0} b_{01}^{4}+b_{05}^{3} D_{0} b_{11}^{5} \not \equiv 0\right)$.

Les conditions L s'écrivent

$b_{01}^{3} \equiv 0$

$\left(b_{04}^{3} \not \equiv 0, b_{01}^{5} \equiv b_{02}^{3}+b_{04}^{5} \equiv\left(b_{02}^{3}\right)^{2}+b_{04}^{3} b_{01}^{4} \equiv 0, C^{\prime} \equiv C_{3}^{\prime} \equiv C_{4}^{\prime} \equiv C_{5}^{\prime} \equiv 0\right)$;

ou

$\left(b_{01}^{5} \not \equiv 0, b_{04}^{3} \equiv b_{02}^{3}+b_{04}^{5} \equiv\left(b_{02}^{3}\right)^{2}+b_{05}^{3} b_{01}^{5} \equiv 0, C^{\prime \prime} \equiv C_{3}^{\prime \prime} \equiv C_{4}^{\prime \prime} \equiv C_{5}^{\prime \prime} \equiv 0\right)$;

ou

$\left(b_{01}^{3} \equiv b_{04}^{3} \equiv b_{02}^{3} \equiv b_{01}^{5}=b_{04}^{5} \equiv b_{11}^{3} \equiv b_{14}^{3} b_{01}^{4}+b_{05}^{3} b_{11}^{5} \equiv 0\right.$,

$\left.b_{05}^{3} b_{14}^{5} b_{01}^{4}+b_{14}^{3} b_{11}^{5}\left|\xi^{\prime}\right|+b_{14}^{3} D_{0} b_{01}^{4}+b_{05}^{3} D_{0} b_{11}^{5} \equiv 0\right)$.

\subsection{Cas $(\mathbf{L G})_{3 / 2}$.}

a) $b_{01}^{3} b_{04}^{5}-b_{04}^{3} b_{01}^{5} \neq 0 . \quad N$ est formé des points $0,(3,2),(5,3)$.

De même qu'au $§ 1.3 \mathrm{a}$ ), on cherche $f$ sous la forme:

$$
\begin{aligned}
f & =\sum_{1 \leq t \leq 3} f_{1}^{t}+\sum_{1 \leq t^{\prime} \leq 2} f_{2}^{t^{\prime}} ; \\
f_{1}^{t} & =e^{\psi_{1}^{t}+\psi_{2}^{t}}\left[Y_{0}^{t}+\cdots Y_{k}^{t}+\cdots\right]
\end{aligned}
$$

$\left(D_{0} \psi_{1}^{t}\right)^{3}=-b_{01}^{3}|\eta|^{2} ; \psi_{1}^{t}$ est d'ordre $\frac{2}{3}, \psi_{2}^{t}$ est d'ordre $\frac{1}{3}$, et est déterminée à partir de $\psi_{1}^{t}, Y_{k}^{t}$ est d'ordre $\frac{4-k}{6}$;

$f_{2}=e^{\chi^{t^{\prime}}}\left[Z_{0}^{t^{\prime}}+\cdots+Z_{k}^{t^{\prime}}+\cdots\right],\left(D_{0} \chi^{t^{\prime}}\right)^{2}=-\frac{b_{01}^{3} b_{04}^{5}-b_{04}^{3} b_{01}^{5}}{b_{01}^{3}}|\eta| ; \chi^{t^{\prime}}$ est d'ordre $\frac{1}{2}$, $Z_{k}^{t^{\prime}}$ est d'ordre $\frac{3-k}{6}$.

Les systèmes en $Y$ et $Z$ sont ceux du $\S 1.3$ a).

On en déduit, en résumant (0) (1) (2) (3) (4) (5)

$$
Y_{0}^{2}=Y_{0}^{3}=Y_{0}^{5}=Y_{0}^{4}=0 ; \quad Y_{1}^{2}=Y_{1}^{3}=Y_{1}^{5}=Y_{1}^{4}=0 ; \quad|\eta| Y_{2}^{2}+D \psi_{1} Y_{0}^{1}=0 ;
$$




$$
\begin{aligned}
& Y_{2}^{3}=Y_{2}^{5}=0 ; \quad Y_{3}^{3}=Y_{3}^{5}=0 ; \quad|\eta| Y_{4}^{2}+\cdots+\left(D_{0} \psi_{2}-\frac{D_{0} \psi_{1} D_{*} \psi_{1}}{|\eta|}\right) Y_{0}^{1}=0 \\
& |\eta| Y_{4}^{3}-\frac{\left(D_{0} \psi_{1}\right)^{2}}{|\eta|} Y_{0}^{1}=0 ; \quad|\eta| Y_{4}^{5}+D_{0} \psi_{1} Y_{2}^{4}=0 ; \quad \ldots
\end{aligned}
$$

à l'étape (6), on a à la $3^{\text {ème }}$ ligne

$$
\left[\left(D_{0} \psi_{1}\right)^{3}+|\eta|^{2} b_{01}^{3}\right] Y_{0}^{1}=0
$$

compte tenu du choix de $\psi_{1}, Y_{0}^{1}$ reste libre; on a aussi

$$
\begin{aligned}
& |\eta| Y_{6}^{2}+\cdots+D_{0} Y_{0}^{1}+W Y_{0}^{1}=0 \\
& |\eta| Y_{6}^{2}+\cdots+\left(-\frac{2 D_{0} \psi_{1} D_{0} \psi_{2}}{|\eta|}+\frac{2\left(D_{0} \psi_{1}\right)^{2} D_{*} \psi_{1}}{|\eta|^{2}}\right) Y_{0}^{1}=0 \\
& \left(D_{0} \psi_{1}\right)^{2} Y_{2}^{4}-b_{01}^{5}|\eta| Y_{0}^{1}=0
\end{aligned}
$$

à l'étape (8), on a à la $3^{\text {ème }}$ ligne

$$
\left[3\left(D_{0} \psi_{1}\right)^{2} D_{0} \psi_{2}+W\left(\psi_{1} ; \frac{5}{3}\right)\right] Y_{0}^{1}=0
$$

on choisit $D_{0} \psi_{2}$ en fonction de $\psi_{1}$, de façon à laisser $Y_{0}^{1}$ libre; on a aussi

$$
|\eta| Y_{8}^{3}-\cdots-\frac{2 D_{0} \psi_{1}}{|\eta|} D_{0} Y_{0}^{1}+W Y_{0}^{1}=0
$$

à l'étape (10), on a à la $3^{\text {ème }}$ ligne:

$$
3\left(D_{0} \psi_{1}\right)^{2} D_{0} Y_{0}^{1}+W Y_{0}^{1}=0
$$

Pour les $Z$, on obtient

$$
Z_{0}^{2}=Z_{0}^{3}=Z_{0}^{5} ; \quad|\eta| Z_{3}^{2}+D_{0} \chi Z_{0}^{1}=0, \quad Z_{3}^{3}=0, \quad|\eta| Z_{3}^{5}+D_{0} \chi Z_{0}^{4}=0, \quad \ldots
$$

à la $6^{\text {ème }}$ étape, on a:

$$
\begin{aligned}
& |\eta| Z_{6}^{3}-\frac{\left(D_{0} \chi\right)^{2}}{|\eta|} Z_{0}^{1}=0, \quad b_{01}^{3} Z_{0}^{1}+b_{04}^{3} Z_{0}^{4}=0 \\
& |\eta| Z_{6}^{5}+D_{0} \chi Z_{3}^{4}+b_{01}^{4} Z_{0}^{1}+D_{0} Z_{0}^{4}=0
\end{aligned}
$$

à la $5^{\text {ème }}$ ligne:

$$
b_{01}^{5} Z_{0}^{1}+\left(-\frac{\left(D_{0} \chi\right)^{2}}{|\eta|}+b_{04}^{5}\right) Z_{0}^{4}=0
$$


le choix de $D_{0} \chi$ laisse $Z_{0}^{4}$ libre; .. ; à la $3^{\text {ème }}$ ligne de la $9^{\text {ème }}$ étape, on a

$$
\left[-\frac{\left(D_{0} \chi\right)^{3}}{|\eta|^{2}} \frac{b_{04}^{3}}{b_{01}^{3}}+\frac{b_{02}^{3} b_{04}^{3} D_{0} \chi}{b_{01}^{3}|\eta|}-\frac{b_{05}^{3} D_{0} \chi}{|\eta|}\right] Z_{0}^{4}+b_{01}^{3} Z_{3}^{1}+b_{04}^{3} Z_{3}^{4}=0
$$

à la $5^{\text {ème }}$ ligne de la $9^{\text {ème }}$ étape:

$$
-\frac{2 D_{0} \chi D_{0} Z_{0}^{4}}{|\eta|}+b_{01}^{5} Z_{3}^{1}+\left(-\frac{\left(D_{0} \chi\right)^{2}}{|\eta|}+b_{04}^{5}\right) Z_{3}^{4}+W Z_{0}^{4}=0
$$

d'où

$$
D_{0} Z_{0}^{4}+W^{\prime} Z_{0}^{4}=0
$$

On impose les conditions initiales

$$
\begin{aligned}
& \sum_{t} Y_{0}^{t}\left|=0 ; \quad \sum_{t} Y_{1}^{t}\right|+\sum_{t^{\prime}} Z_{0}^{t^{\prime}}\left|=0 ; \quad \sum_{t} Y_{2}^{t}\right|+\sum_{t^{\prime}} Z_{1}^{t^{\prime}} \mid=0 ; \\
& \sum_{t} Y_{3}^{t}\left|+\sum_{t^{\prime}} Z_{2}^{t^{\prime}}\right|=0 ; \quad \sum_{t} Y_{4}^{t}\left|+\sum_{t^{\prime}} Z_{3}^{t^{\prime}}\right|=I ; \quad \ldots ;
\end{aligned}
$$

on obtient

$$
\begin{aligned}
& \sum_{t} Y_{0}^{t 1}\left|=0 ; \quad \sum_{t^{\prime}} Z_{0}^{t^{\prime} 4}\right|=0 ; \quad \sum_{t} D_{0} \psi^{t} Y_{0}^{t 1} \mid=0 \\
& \sum_{t} \frac{\left(D_{0} \psi_{1}^{t}\right)^{2} Y_{0}^{t 1} \mid}{|\eta|^{2}}=I^{3} \\
& \sum_{t^{\prime}} D_{0} \chi_{1}^{t} Z_{0}^{t^{\prime} 4}|=-| \eta\left|I^{5}-\frac{b_{01}^{5} \mid}{b_{01}^{3} \mid}\right| \eta \mid I^{3}
\end{aligned}
$$

on en déduit les $Y_{0}^{t 1} \mid$ et les $Z_{0}^{t^{\prime}}{ }^{4} \mid$, d'où les $Y_{0}^{t 1}$ et les $Z_{0}^{t^{\prime} 4}$ et tous les coefficients.

b) $b_{01}^{3} b_{04}^{5}-b_{04}^{3} b_{01}^{5}=0 . \quad N$ est formé des points $0,(3,2),(4,2)$ et $(5,2)$.

De même qu'au $\S 1.3 \mathrm{~b}$ ), on cherche $f$ sous la forme

$$
\begin{aligned}
f & =\sum_{1 \leq t \leq 3} f_{1}^{t}+f_{2} ; \\
f_{1}^{t} & =e^{\psi_{1}^{t}+\psi_{2}^{t}}\left[Y_{0}^{t}+\cdots+Y_{k}^{t}+\cdots\right] ;
\end{aligned}
$$

$\left(D_{0} \psi^{t}\right)^{3}=-b_{01}^{3}|\eta|^{2} ; \psi_{1}^{t}$ est d'ordre $\frac{2}{3}, \psi_{2}^{t}$ est d'ordre $\frac{1}{3}$, et sa détermination sera expliquée ci-dessous; $Y_{k}^{t}$ est d'ordre $\frac{2-k}{3}$;

$$
f_{2}=Z_{0}+\cdots+Z_{k}+\cdots ; Z_{k} \text { est d'ordre } \frac{3-k}{3} \text {. }
$$


Les systèmes différentiels en $Y$ et $Z$ sont les mêmes que ceux des cas cité ci-dessus.

On en déduit

$$
\begin{aligned}
& Y_{0}^{2}=Y_{0}^{3}=Y_{0}^{5}=0 ; \quad|\eta| Y_{1}^{2}+D_{0} \psi_{1} Y_{0}^{1}=0 ; \quad Y_{1}^{3}=Y_{1}^{5}=0 \\
& |\eta| Y_{2}^{2}+\cdots+\left(\frac{-D_{0} \psi_{1} D_{*} \psi_{1}}{|\eta|}+D_{0} \psi_{2}\right) Y_{0}^{1}=0 ; \quad|\eta| Y_{2}^{3}-\frac{\left(D_{0} \psi_{1}\right)^{2}}{|\eta|} Y_{0}^{1}=0 \\
& |\eta| Y_{2}^{5}+D_{0} \psi_{1} Y_{1}^{4}=0 ; \quad Y_{1}^{5}=Y_{0}^{4}=0
\end{aligned}
$$

à la $3^{\text {ème }}$ étape, à la $3^{\text {ème }}$ ligne:

$$
\left[\frac{\left(D_{0} \psi_{1}\right)^{3}}{|\eta|^{2}}+b_{01}^{3}\right] Y_{0}^{1}=0
$$

qui laisse $Y_{0}^{1}$ libre, à la $5^{\text {ème }}$ ligne:

$$
\begin{aligned}
& Y_{1}^{4}=\frac{b_{01}^{5}|\eta|}{\left(D_{0} \psi_{1}\right)^{2}} Y_{0}^{1} \\
& |\eta| Y_{3}^{2}+\cdots+D_{0} Y_{0}^{1}+\left[\frac{D_{0} \psi_{1}\left(D_{*} \psi_{1}\right)^{2}}{|\eta|^{2}}-\frac{D_{0} \psi_{1} D_{*} \psi_{2}}{|\eta|}-\frac{D_{0} \psi_{2} D_{*} \psi_{1}}{|\eta|}\right] Y_{0}^{1}=0 \\
& |\eta| Y_{3}^{3}+\cdots+\left[\frac{2\left(D_{0} \psi_{1}\right)^{2} D_{*} \psi_{1}}{|\eta|^{2}}-\frac{2 D_{0} \psi_{1} D_{0} \psi_{2}}{|\eta|}\right] Y_{0}^{1}=0 \\
& |\eta| Y_{3}^{5}+D_{0} \psi_{1} Y_{2}^{4}+\left[-\frac{D_{*} \psi_{1} D_{0} \psi_{1}}{|\eta|}+D_{0} \psi_{2}\right] Y_{1}^{4}+b_{01}^{4} Y_{0}^{1}=0
\end{aligned}
$$

à la $4^{\text {ème }}$ étape, on a à la $3^{\text {ème }}$ ligne:

$$
\left[-\frac{2\left(D_{0} \psi_{1}\right)^{3} D_{*} \psi_{1}}{|\eta|^{3}}+\frac{3\left(D_{0} \psi_{1}\right)^{2} D_{0} \psi_{2}}{|\eta|^{2}}-\frac{b_{02}^{3} D_{0} \psi_{1}}{|\eta|}+\frac{b_{04}^{3} b_{01}^{5}|\eta|}{\left(D_{0} \psi_{1}\right)^{2}}\right] Y_{0}^{1}=0
$$

qui détermine $D_{0} \psi_{2} ;$ on a aussi

$$
\begin{gathered}
Y_{2}^{4}=\cdots+W Y_{0}^{1} \\
|\eta| Y_{4}^{3}-\frac{2 D_{0} \psi_{1} D_{0} Y_{0}^{1}}{|\eta|}+W^{\prime} Y_{0}^{1}=0
\end{gathered}
$$

à la $5^{\text {ème }}$ étape, à la $3^{\text {ème }}$ ligne:

$$
3 \frac{\left(D_{0} \psi_{1}\right)^{2} D_{0} Y_{0}^{1}}{|\eta|}+W^{\prime \prime} Y_{0}^{1}=0
$$

il reste à déterminer $Y_{0}^{1} \mid$. 
Le système différentiel des $Z$ donne

$$
\begin{array}{ll}
Z_{0}^{2}=Z_{0}^{3}=Z_{0}^{5}=0 ; & Z_{1}^{2}=Z_{1}^{3}=Z_{1}^{5}=0 \\
Z_{2}^{2}=Z_{2}^{3}=Z_{2}^{5}=0 ; & b_{01}^{3} Z_{0}^{1}+b_{04}^{3} Z_{0}^{4}=0 ; \\
|\eta| Z_{3}^{2}+D_{0} Z_{0}^{1}=0 ; & Z_{3}^{3}=0 ; \quad|\eta| Z_{3}^{5}+D_{0} Z_{0}^{4}+b_{01}^{4} Z_{0}^{1}=0 ; \quad \ldots ;
\end{array}
$$

à la $6^{\text {ème }}$ étape, la $3^{\text {ème }}$ ligne s'écrit:

$$
\begin{gathered}
b_{01}^{3} Z_{3}^{1}+b_{04}^{3} Z_{3}^{4}-\frac{b_{02}^{3}}{|\eta|} D_{0} Z_{0}^{1}-\frac{b_{05}^{3} D_{0} Z_{0}^{4}}{|\eta|}+\left(-\frac{b_{05}^{3} b_{01}^{4}}{|\eta|}+b_{11}^{3}\right) Z_{0}^{1} \\
+b_{14}^{3} Z_{0}^{4}+\left(D b_{01}^{3}\right)_{*}\left(Z_{0}^{1}\right)+\left(D b_{04}^{3}\right)_{*}\left(Z_{0}^{4}\right)=0
\end{gathered}
$$

la $5^{\text {ème }}$ ligne s'écrit:

$$
\begin{gathered}
b_{01}^{5} Z_{3}^{1}+b_{04}^{5} Z_{3}^{4}-\frac{D_{0}^{2} Z_{0}^{4}}{|\eta|}-\frac{b_{01}^{4}}{|\eta|} D_{0} Z_{0}^{1}+\left(b_{11}^{5}-\frac{D_{0} b_{01}^{4}}{|\eta|}\right) Z_{0}^{1} \\
+b_{14}^{5} Z_{0}^{4}+\left(D b_{01}^{5}\right)_{*}\left(Z_{0}^{1}\right)+\left(D b_{04}^{5}\right)_{*}\left(Z_{0}^{4}\right)=0
\end{gathered}
$$

on en déduit

$$
\frac{D_{0}^{2} Z_{0}^{4}}{|\eta|}+W D_{0} Z_{0}^{4}+W^{\prime} Z_{0}^{4}=0
$$

il reste à déterminer $Z_{0}^{4} \mid$ et $D_{0} Z_{0}^{4} \mid$.

On impose les conditions initiales:

$$
Z_{0}\left|=0 ; \quad \sum_{t} Y_{0}^{t}\right|+Z_{1}\left|=0 ; \quad \sum_{t} Y_{1}^{t}\right|+Z_{2}\left|=0 ; \quad \sum_{t} Y_{2}^{t}\right|+Z_{3} \mid=I ; \quad \cdots
$$

on en déduit

$$
Z_{0}^{4}\left|=0 ; \quad \sum_{t} Y_{0}^{t 1}\right|=0 ; \quad \sum_{t} D_{0} \psi_{1}^{t} Y_{0}^{t 1}\left|=0 ; \quad \sum_{t}\left(D_{0} \psi_{1}^{t}\right)^{2} Y_{0}^{t 1}\right|=|\eta|^{2} I^{3}
$$

d'où l'on détermine les $Y_{0}^{t 1} \mid$,

$$
\frac{b_{01}^{5}}{b_{01}^{3}} \sum_{t}\left(D_{0} \psi_{1}^{t}\right) Y_{0}^{t 1}|-| \eta\left|D_{0} Z_{0}^{4}\right|=|\eta|^{2} I^{5}
$$

d'où l'on détermine les $D_{0} Z_{0}^{4} \mid$. 
3.2. Cas $(\mathbf{L G})_{5 / 3}$. $N$ est formé des point 0 et $(5,3)$. De même qu'au $\S 1.4$, on cherche $f$ sous la forme

$$
f=\sum_{t} e^{\psi_{1}^{t}+\psi_{2}^{t}+\psi_{3}^{t}}\left[Y_{0}^{t}+\cdots Y_{k}^{t}+\cdots\right]
$$

$\left(D_{0} \psi_{1}^{t}\right)^{5}=-b_{04}^{3} b_{01}^{5}|\eta|^{3} ; \psi_{1}^{t}$ est d'ordre $\frac{3}{5}, \psi_{2}^{t}$ est d'ordre $\frac{2}{5}, \psi_{3}^{t}$ est d'ordre $\frac{1}{5}$, et ils seront précisées ci-dessous; $Y_{k}^{t}$ est d'ordre $\frac{4-k}{5}$.

Le système différentiel en $Y$ est le même que celui du cas cité ci-dessus.

On en déduit

$$
\begin{aligned}
& Y_{0}^{2}=Y_{0}^{3}=Y_{0}^{5}=0 ; \quad Y_{1}^{2}=Y_{1}^{3}=Y_{1}^{5}=0 ; \quad|\eta| Y_{2}^{2}+D_{0} \psi_{1} Y_{0}^{1}=0, \quad Y_{2}^{3}=0 \\
& |\eta| Y_{2}^{5}+D_{0} \psi_{1} Y_{0}^{4}=0 ; \quad|\eta| Y_{3}^{2}+\cdots+D_{0} \psi_{2} Y_{0}^{1}=0 ; \quad Y_{3}^{3}=0 \\
& |\eta| Y_{3}^{5}+D_{0} \psi_{1} Y_{1}^{4}+D_{0} \psi_{2} Y_{0}^{4}=0 ; \quad|\eta| Y_{4}^{2}+\cdots+\left[-\frac{D_{*} \psi_{1} D_{0} \psi_{1}}{|\eta|}+D_{0} \psi_{3}\right] Y_{0}^{1}=0 \\
& |\eta| Y_{4}^{3}-\frac{\left(D_{0} \psi_{1}\right)^{2}}{|\eta|} Y_{0}^{1}=0 ; \quad Y_{0}^{4}=0 ; \quad|\eta| Y_{4}^{5}+D_{0} \psi_{1} Y_{2}^{4}+D_{0} \psi_{2} Y_{1}^{4}=0 \\
& Y_{1}^{4}=\frac{b_{01}^{5}|\eta|}{\left(D_{0} \psi_{1}\right)^{2}} Y_{0}^{1} ; \\
& |\eta| Y_{5}^{2}+\cdots+D_{0} Y_{0}^{1}+\left[-\frac{D_{*} \psi_{1} D_{0} \psi_{2}}{|\eta|}-D_{*} \psi_{2} D_{0} \psi_{1}\right] Y_{0}^{1}=0 \\
& |\eta| Y_{5}^{3}+\cdots-2 \frac{D_{0} \psi_{1} D_{0} \psi_{2}}{|\eta|} Y_{0}^{1}=0 \\
& |\eta| Y_{5}^{5}+D_{0} \psi_{1} Y_{3}^{4}+D_{0} \psi_{2} Y_{2}^{4}+\left[-\frac{D_{*} \psi_{1} D_{0} \psi_{1}}{|\eta|}+D_{0} \psi_{3}\right] Y_{1}^{4}+b_{01}^{4} Y_{0}^{1}=0
\end{aligned}
$$

à la $6^{\text {ème }}$ étape, la $3^{\text {ème }}$ ligne donne

$$
\left[\frac{\left(D_{0} \psi_{1}\right)^{3}}{|\eta|^{2}}+\frac{b_{04}^{3} b_{01}^{5}|\eta|}{\left(D_{0} \psi_{1}\right)^{2}}\right] Y_{0}^{1}=0
$$

qui laisse $Y_{0}^{1}$ libre, la $5^{\text {ème }}$ ligne donne:

$$
Y_{2}^{4}=\cdots\left(-2 D_{0} \psi_{1} D_{0} \psi_{2}+b_{04}^{5}|\eta|\right) \frac{b_{01}^{5}|\eta|}{\left(D_{0} \psi_{1}\right)^{4}} Y_{0}^{1}
$$

on a aussi

$$
\begin{aligned}
& |\eta| Y_{6}^{3}+\cdots+\left(2 \frac{\left(D_{0} \psi_{1}\right)^{2} D_{*} \psi_{1}}{|\eta|^{2}}-2 \frac{D_{0} \psi_{1} D_{0} \psi_{3}}{|\eta|}-\frac{\left(D_{0} \psi_{2}\right)^{2}}{|\eta|}\right) Y_{0}^{1}=0 \\
& |\eta| Y_{6}^{5}+\cdots+\frac{b_{01}^{5}|\eta|}{\left(D_{0} \psi_{1}\right)^{2}} D_{0} Y_{0}^{1}+W Y_{0}^{1}=0
\end{aligned}
$$


à la $7^{\text {ème }}$ étape, la $3^{\text {ème }}$ ligne détermine $D_{0} \psi_{2}$ :

$$
5 D_{0} \psi_{1} D_{0} \psi_{2}=\left(b_{02}^{3}+b_{04}^{5}\right)|\eta|
$$

la $5^{\text {ème }}$ ligne donne

$$
\begin{gathered}
Y_{3}^{4}=\cdots+\left[-\frac{2 D_{0} \psi_{3}|\eta| b_{01}^{5}}{\left(D_{0} \psi_{1}\right)^{3}}+W\left(\psi_{1}, \psi_{2}\right)\right] Y_{0}^{1} \\
|\eta| Y_{7}^{3}+\cdots-\frac{2 D_{0} \psi_{1} D_{0} Y_{0}^{1}}{|\eta|}+W^{\prime} Y_{0}^{1}=0
\end{gathered}
$$

la $3^{\text {ème }}$ ligne de la $8^{\text {ème }}$ étape détermine $D_{0} \psi_{3}$; la $5^{\text {ème }}$ ligne donne:

$$
Y_{4}^{4}=\cdots-\frac{2 b_{01}^{5}|\eta|}{\left(D_{0} \psi_{1}\right)^{3}} D_{0} Y_{0}^{1}+W^{\prime \prime} Y_{0}^{1}
$$

la $3^{\text {ème }}$ ligne de la $9^{\text {ème }}$ étape donne:

$$
D_{0} Y_{0}^{1}+W^{\prime \prime \prime} Y_{0}^{1}=0
$$

il reste à déterminer $Y_{0}^{1} \mid$.

On impose

$$
\sum_{t} Y_{0}^{t}\left|=\sum_{t} Y_{1}^{t}\right|=\sum_{t} Y_{2}^{t}\left|=\sum_{t} Y_{3}^{t}\right|=0 ; \quad \sum_{t} Y_{4}^{t} \mid=I ; \quad \cdots
$$

On a alors

$$
\begin{aligned}
& \sum_{t} Y_{0}^{t 1}\left|=0 ; \quad \sum_{t}\left(D_{0} \psi_{1}^{t}\right)^{3} Y_{0}^{t 1}\right|=0 ; \quad \sum_{t} D_{0} \psi_{1}^{t} Y_{0}^{t 1} \mid=0 ; \\
& \sum_{t} D_{0}\left(\psi_{1}^{t}\right)^{4} Y_{0}^{t 1}\left|=0 ; \quad \sum_{t} D_{0}\left(\psi_{1}^{t}\right)^{2} Y_{0}^{t 1}\right|=|\eta|^{2} I^{3} ; \quad \ldots
\end{aligned}
$$

On obtient ainsi $Y_{0}^{t 1} \mid$ et $Y_{0}^{t 1}, Y_{0}^{t}$ et tous les $Y_{k}^{t}$.

\subsection{Cas (LG) $)_{2}$.}

1) $b_{04}^{3} b_{01}^{4}+b_{05}^{3} b_{01}^{5}-b_{02}^{3} b_{04}^{5} \neq 0 . \quad N$ est formé des point $0,(2,1),(4,2)$. Comme au $\S 1.5$ on distingue encore:

a) $\left(b_{02}^{3}+b_{04}^{5}\right)^{2}+4\left(b_{04}^{3} b_{01}^{4}+b_{05}^{3} b_{01}^{5}-b_{02}^{3} b_{04}^{5}\right) \neq 0$. 
$\left.\mathbf{a}_{1}\right) b_{04}^{3}$ ou $b_{01}^{5} \neq 0$.

$$
\begin{aligned}
f & =\sum_{1 \leq t \leq 2} f_{1}^{t}+f_{2} ; \\
f_{1}^{t} & =e^{\psi^{t}}\left[Y_{0}^{t}+\cdots Y_{k}^{t}+\cdots\right]
\end{aligned}
$$

$\left(D_{0} \psi^{t}\right)$ est une des quatre racine distinctes de l'équation

$$
\left(D_{0} \psi^{t}\right)^{4}-|\eta|\left(b_{02}^{3}+b_{04}^{5}\right)\left(D_{0} \psi^{t}\right)^{2}+\left(b_{04}^{3} b_{01}^{4}+b_{05}^{3} b_{01}^{5}-b_{02}^{3} b_{04}^{5}\right)|\eta|^{2}=0 ;
$$

$D_{0} \psi^{t}$ est d'ordre $\frac{1}{2}, Y_{k}^{t}$ est d'ordre $\frac{2-k}{2}$;

$$
f_{2}=Z_{0}^{t}+\cdots+Z_{k}+\cdots
$$

$Z_{k}$ est d'ordre $\frac{2-k}{2}$.

Les systèmes différentiels en $Y$ et $Z$ sont les mêmes que ceux du cas cité.

Pour mener à bien les calculs, on doit distinguer

a) ${ }_{1}, b_{04}^{3} \neq 0$.

(0) $Y_{0}^{2}=Y_{0}^{3}=Y_{0}^{5}=0$;

(1) $|\eta| Y_{1}^{2}+D_{0} \psi Y_{0}^{1}=0 ; \quad Y_{1}^{3}=0 ; \quad|\eta| Y_{1}^{5}+D_{0} \psi Y_{0}^{4}=0$

(2) $|\eta| Y_{2}^{2}+\cdots+D_{0} Y_{0}^{1}-\frac{D_{*} \psi D_{0} \psi}{|\eta|} Y_{0}^{1}=0 ; \quad|\eta| Y_{2}^{3}-\frac{\left(D_{0} \psi\right)^{2}}{|\eta|} Y_{0}^{1}=0 ; \quad Y_{0}^{4}=0$;

$|\eta| Y_{2}^{5}+D_{0} \psi Y_{1}^{4}+b_{01}^{4} Y_{0}^{1}=0 ;$

(3) $|\eta| Y_{3}^{2}+\cdots-\frac{D_{*} \psi D_{0} Y_{0}^{1}}{|\eta|}-\frac{D_{0} \psi D_{*} Y_{0}^{1}}{|\eta|} Y_{0}^{1}+\left[\frac{\left(D_{*} \psi\right)^{2} D_{0} \psi}{|\eta|^{2}}-\frac{D_{* 0}^{2} \psi}{|\eta|}\right] Y_{0}^{1}=0$;

$$
\begin{aligned}
& |\eta| Y_{3}^{3}+\cdots-\frac{2 D_{0} \psi D_{0} Y_{0}^{1}}{|\eta|}+\left[\frac{2\left(D_{0} \psi\right)^{2} D_{*} \psi}{|\eta|^{2}}-\frac{D_{0}^{2} \psi}{|\eta|}\right] Y_{0}^{1}=0 \\
& b_{04}^{3} Y_{1}^{4}+D_{0} \psi\left[\frac{\left(D_{0} \psi\right)^{2}}{|\eta|^{2}}-\frac{b_{02}^{3}}{|\eta|}\right] Y_{0}^{1}=0
\end{aligned}
$$

à la $5^{\text {ème }}$ ligne

$$
\left[-\frac{\left(D_{0} \psi\right)^{2}}{|\eta|}+b_{04}^{5}\right] Y_{1}^{4}-\frac{D_{0} \psi b_{01}^{4}}{|\eta|} Y_{0}^{1}=0
$$

d'où

$$
\left[\left(D_{0} \psi\right)^{4}-\left.\left(D_{0} \psi\right)^{2}\left(b_{02}^{3}+b_{04}^{5}\right)\left|\eta+\left(b_{02}^{3} b_{04}^{5}-b_{04}^{3} b_{01}^{4}\right)\right| \eta\right|^{2}\right] Y_{0}^{1}=0
$$


qui est réalisée sans condition sur $Y_{0}^{1}$.

$$
\begin{gathered}
|\eta| Y_{3}^{5}+D_{0} \psi Y_{2}^{4}+b_{01}^{4} Y_{1}^{1}+D_{0} Y_{1}^{4}-\frac{D_{*} \psi D_{0} \psi}{|\eta|} Y_{1}^{4} \\
+\left(D b_{01}^{4}\right)_{*}(\psi) Y_{0}^{1}-D_{*} \psi \frac{b_{01}^{4}}{|\eta|} Y_{0}^{1}=0
\end{gathered}
$$

(4) $|\eta| Y_{4}^{3}+\cdots+\frac{4 D_{0} \psi D_{*} \psi}{|\eta|^{2}} D_{0} Y_{0}^{1}+\frac{2\left(D_{0} \psi\right)^{2}}{|\eta|^{2}} D_{*} Y_{0}^{1}-\frac{D_{0}^{2} Y_{0}^{1}}{|\eta|}+W Y_{0}^{1}=0$

$$
\begin{aligned}
& |\eta| Y_{4}^{5}+\cdots+D_{0} \psi Y_{3}^{4}+D_{0} Y_{2}^{4}-\frac{D_{0} \psi}{|\eta|} D_{*} Y_{1}^{4}-\frac{b_{01}^{4}}{|\eta|} D_{*} Y_{0}^{1}-\frac{D_{*} \psi}{|\eta|} D_{0} Y_{1}^{4} \\
& +\left(D b_{01}^{4}\right)_{*}\left(Y_{0}^{1}\right)+W^{\prime} Y_{2}^{4}+W^{\prime \prime} Y_{1}^{4}+W^{\prime \prime \prime} Y_{0}^{1}=0
\end{aligned}
$$

à la $3^{\text {ème }}$ ligne

$$
\begin{aligned}
& b_{04}^{3} Y_{2}^{4}+\cdots+\left[\frac{3\left(D_{0} \psi\right)^{2}}{|\eta|^{2}}-\frac{b_{02}^{3}}{|\eta|}\right] D_{0} Y_{0}^{1}+\left[\frac{b_{05}^{3}\left(D_{0} \psi\right)^{4}}{b_{04}^{3}|\eta|^{3}}-\frac{b_{02}^{3} b_{05}^{3}}{b_{04}^{3}|\eta|^{2}}\left(D_{0} \psi\right)^{2}\right. \\
& -\frac{2\left(D_{0} \psi\right)^{3} D_{*} \psi}{|\eta|^{3}}+b_{11}^{3}-\frac{b_{05}^{3} b_{01}^{4}}{|\eta|}+\frac{3 D_{0} \psi D_{0}^{2} \psi}{|\eta|^{2}}+\frac{b_{02}^{3} D_{*} \psi D_{0} \psi}{|\eta|^{2}} \\
& \left.-\left(D b_{02}^{3}\right)_{*}(\psi) \frac{D_{0} \psi}{|\eta|}-\left(D b_{04}^{3}\right)_{*}(\psi) \frac{D_{0} \psi}{b_{04}^{3}}\left(\frac{\left(D_{0} \psi\right)^{2}}{|\eta|^{2}}-\frac{b_{02}^{3}}{|\eta|}\right)\right] Y_{0}^{1}=0
\end{aligned}
$$

à la $5^{\text {ème }}$ ligne

$$
\begin{aligned}
& {\left[-\frac{\left(D_{0} \psi\right)^{2}}{|\eta|}+b_{04}^{5}\right] Y_{2}^{4}+\cdots+\left[\frac{2\left(D_{0} \psi\right)^{2}}{|\eta|^{3}} \frac{\left[\left(D_{0} \psi\right)^{2}-b_{02}^{3}|\eta|\right]}{b_{04}^{3}}-\frac{b_{01}^{4}}{|\eta|}\right] D_{0} Y_{0}^{1}} \\
& +\left\{\frac{2 D_{0} \psi}{|\eta|} D_{0}\left[\frac{D_{0} \psi}{b_{04}^{3}}\left(\frac{\left(D_{0} \psi\right)^{2}-b_{02}^{3}|\eta|}{|\eta|^{2}}\right)\right]+\frac{D_{0}^{2} \psi D_{0} \psi}{|\eta| b_{04}^{3}}\left(\frac{\left(D_{0} \psi\right)^{2}}{|\eta|^{2}}-\frac{b_{02}^{3}}{|\eta|}\right)-\frac{D_{0} b_{01}^{4}}{|\eta|}\right. \\
& \quad+b_{11}^{5}-\frac{\left(D_{0} \psi\right)^{3} D_{*} \psi}{b_{04}^{3}|\eta|^{4}}\left[\left(D_{0} \psi\right)^{2}-|\eta| b_{02}^{3}\right] \\
& \left.\quad+\frac{D_{0} \psi D_{*} \psi}{|\eta|^{2}} b_{01}^{4}-\frac{D_{0} \psi}{|\eta|}\left(D b_{01}^{4}\right)_{*}(\psi)-\left(D b_{04}^{5}\right)_{*}(\psi) \frac{D_{0} \psi}{b_{04}^{3}|\eta|^{2}}\left[\left(D_{0} \psi\right)^{2}-|\eta| b_{02}^{3}\right]\right\} Y_{0}^{1} \\
& =0
\end{aligned}
$$

on en déduit

$$
\begin{aligned}
& \left\{\left(\frac{\left(D_{0} \psi\right)^{2}}{|\eta|}-b_{04}^{5}\right)\left(\frac{3\left(D_{0} \psi\right)^{2}}{|\eta|^{2}}-\frac{b_{02}^{3}}{|\eta|}\right)\right. \\
& \left.+\frac{2\left(D_{0} \psi\right)^{2}}{|\eta|^{3}}\left[\left(D_{0} \psi\right)^{2}-|\eta| b_{02}^{3}\right]-\frac{b_{04}^{3} b_{01}^{4}}{|\eta|}\right\} D_{0} Y_{0}^{1}+C_{1} Y_{0}^{1}=0
\end{aligned}
$$

$C_{1}$ sera précisé dans le cas $\left.2 \mathrm{~b}\right)$. 
Le coefficient de $D_{0} Y_{0}^{1}$ est

$$
\begin{aligned}
\frac{5\left(D_{0} \psi\right)^{4}-3\left(D_{0} \psi\right)^{2}\left(b_{02}^{3}+b_{04}^{5}\right)|\eta|+\left(b_{04}^{5} b_{02}^{3}-b_{04}^{3} b_{01}^{4}\right)|\eta|^{2}}{|\eta|^{3}} \\
=\frac{2\left(D_{0} \psi\right)^{2}\left[2\left(D_{0} \psi\right)^{2}-\left(b_{02}^{3}+b_{04}^{5}\right)|\eta|\right]}{|\eta|^{3}}
\end{aligned}
$$

compte tenu de la condition du discriminant du cas 1a), il n'est pas nul; il reste donc à déterminer $Y_{0}^{1} \mid$.

Pour les $Z$ on obtient

(0) (1) $Z_{0}^{2}=Z_{0}^{3}=Z_{0}^{5}=Z_{1}^{2}=Z_{1}^{3}=Z_{1}^{5}=0$;

(2) $|\eta| Z_{2}^{2}+D_{0} Z_{0}^{1}=0 ; \quad Z_{2}^{3}=0 ; \quad Z_{0}^{4}=0 ; \quad|\eta| Z_{2}^{5}+b_{01}^{4} Z_{0}^{1}=0 ;$

(3) $Z_{1}^{4}=0$;

(4) $b_{04}^{3} Z_{2}^{4}-\frac{b_{02}^{3} D_{0} Z_{0}^{1}}{|\eta|}+\left[-\frac{b_{05}^{3} b_{01}^{4}}{|\eta|}+b_{11}^{3}\right] Z_{0}^{1}=0$

$$
b_{04}^{5} Z_{2}^{4}-\frac{b_{01}^{4} D_{0} Z_{0}^{1}}{|\eta|}+\left[-\frac{D_{0} b_{01}^{4}}{|\eta|}+b_{11}^{5}\right] Z_{0}^{1}=0
$$

d'où

$$
\left(b_{04}^{5} b_{02}^{3}-b_{04}^{3} b_{01}^{4}\right) D_{0} Z_{0}^{1}+\left(b_{05}^{3} b_{04}^{5} b_{01}^{4}-b_{11}^{3} b_{04}^{5}|\eta|-b_{04}^{3} D_{0} b_{01}^{4}+b_{04}^{3} b_{11}^{5}|\eta|\right) Z_{0}^{1}=0
$$

le coefficient de $D_{0} Z_{0}^{1}$ n'est pas nul, dans le cas 1 ); il reste à déterminer $Z_{0}^{1} \mid$.

On impose les conditions initiales:

$$
\sum_{t} Y_{0}^{t}\left|+Z_{0}\right|=0 ; \quad \sum_{t} Y_{1}^{t}\left|+Z_{1}\right|=0 ; \quad \sum_{t} Y_{2}^{t}\left|+Z_{2}\right|=I ; \quad \cdots
$$

on en déduit

$$
\begin{array}{ll}
\sum_{t} Y_{0}^{t 1}\left|+Z_{0}^{1}\right|=0 ; & \sum_{t} D_{0} \psi^{t} Y_{0}^{t 1} \mid=0 \\
\sum_{t}\left(D_{0} \psi^{t}\right)^{3} Y_{0}^{t 1} \mid=0 ; & \left.\sum_{t}\left(D_{0} \psi^{t}\right)^{2} Y_{0}^{t 1}|=| \eta\right|^{2} I^{3} \\
-b_{02}^{3}\left|I^{3}+\left(b_{02}^{3}+b_{04}^{5}\right)\right| I^{3}+\frac{\left(b_{04}^{3} b_{01}^{4}-b_{04}^{5} b_{02}^{3}\right)}{|\eta|} \sum_{t} Y_{0}^{t 1}\left|=b_{04}^{3}\right| I^{5}
\end{array}
$$

on obtient donc les $Y_{0}^{t 1} \mid$ puis $Z_{0}^{1} \mid$ et tous les coefficients. 
a) $1^{\prime \prime} b_{01}^{5} \neq 0$.

(0) $Y_{0}^{2}=Y_{0}^{3}=Y_{0}^{5}=0$;

(1) $|\eta| Y_{1}^{2}+D_{0} \psi Y_{0}^{1}=0 ; \quad Y_{1}^{3}=0 ; \quad|\eta| Y_{1}^{5}+D_{0} \psi Y_{0}^{4}=0 ;$

(2) $|\eta| Y_{2}^{2}+D_{0} \psi Y_{1}^{1}+D_{0} Y_{0}^{1}-\frac{D_{*} \psi D_{0} \psi Y_{0}^{1}}{|\eta|}=0 ; \quad|\eta| Y_{2}^{3}-\frac{\left(D_{0} \psi\right)^{2}}{|\eta|} Y_{0}^{1}=0$; $\left[\frac{b_{04}^{5}|\eta|-\left(D_{0} \psi\right)^{2}}{|\eta|}\right] Y_{0}^{4}+b_{01}^{5} Y_{0}^{1}=0$ $|\eta| Y_{2}^{5}+D_{0} \psi Y_{1}^{4}+D_{0} Y_{0}^{4}+b_{01}^{4} Y_{0}^{1}-\frac{D_{*} \psi D_{0} \psi Y_{0}^{4}}{|\eta|}=0$

(3) à la $3^{\text {ème }}$ ligne

$$
\left[\left(D_{0} \psi\right)^{2}-b_{02}^{3}|\eta|\right] Y_{0}^{1}-b_{05}^{3}|\eta| Y_{0}^{4}=0
$$

on en déduit

$$
\left[\left(D_{0} \psi\right)^{4}-\left(D_{0} \psi\right)^{2}\left(b_{02}^{3}+b_{04}^{5}\right)|\eta|+\left(b_{02}^{3} b_{04}^{5}-b_{05}^{3} b_{01}^{5}\right)|\eta|^{2}\right] Y_{0}^{4}=0,
$$

qui est réalisée sans condition sur $Y_{0}^{4}$; à la $5^{\text {ème }}$ ligne

$$
\begin{aligned}
& {\left[\frac{b_{04}^{5}|\eta|-\left(D_{0} \psi\right)^{2}}{|\eta|}\right] Y_{1}^{4}+b_{01}^{5} Y_{1}^{1}-\frac{2 D_{0} \psi D_{0} Y_{0}^{4}}{|\eta|}+\left[-\frac{\left(D_{0} \psi\right)^{3} b_{01}^{4}}{|\eta|^{2} b_{01}^{5}}+\frac{D_{0} \psi b_{04}^{5} b_{01}^{4}}{|\eta| b_{01}^{5}}\right.} \\
& \left.-\frac{D_{0}^{2} \psi}{|\eta|}+\frac{2\left(D_{0} \psi\right)^{2} D_{*} \psi}{|\eta|^{2}}+\left(D b_{04}^{5}\right)_{*}(\psi)-\left(D b_{01}^{5}\right)_{*}(\psi) \frac{b_{04}^{5}|\eta|-\left(D_{0} \psi\right)^{2}}{b_{01}^{5}|\eta|}\right] Y_{0}^{4}=0
\end{aligned}
$$

on a aussi

$$
\begin{gathered}
|\eta| Y_{3}^{3}-\frac{\left(D_{0} \psi\right)^{2}}{|\eta|} Y_{1}^{1}-\frac{2 D_{0} \psi}{|\eta|} D_{0} Y_{0}^{1}+\left[\frac{2\left(D_{0} \psi\right)^{2} D_{1} \psi}{|\eta|^{2}}-\frac{D_{0}^{2} \psi}{|\eta|}\right] Y_{0}^{1}=0 \\
|\eta| Y_{3}^{5}+D_{0} \psi Y_{2}^{4}+D_{0} Y_{1}^{4}-\frac{D_{*} \psi D_{0} \psi}{|\eta|} Y_{1}^{4}+b_{01}^{4} Y_{1}^{1}-\frac{D_{*} \psi}{|\eta|} D_{0} Y_{0}^{4}-\frac{D_{0} \psi}{|\eta|} D_{*} Y_{0}^{4} \\
-\frac{D_{*} \psi b_{01}^{4}}{|\eta|} Y_{0}^{1}+\left[\frac{\left(D_{*} \psi\right)^{2} D_{0} \psi}{|\eta|^{2}}-\frac{D_{* 0}^{2} \psi}{|\eta|}\right] Y_{0}^{4}+\left(D b_{01}^{4}\right)_{*}(\psi) Y_{0}^{1}=0
\end{gathered}
$$

(4) à la $3^{\text {ème }}$ ligne on obtient, en tenant compte de ce qui précède:

$$
\frac{2\left(D_{0} \psi\right)^{2}\left[2\left(D_{0} \psi\right)^{2}-\left(b_{02}^{3}+b_{04}^{5}\right)|\eta|\right]}{|\eta|^{3}} D_{0} Y_{0}^{4}+C_{2} Y_{0}^{4}=0
$$

il reste à déterminer $Y_{0}^{4} \mid$; on aura besoin, dans le cas suivant, de la $5^{\text {ème }}$ ligne:

$$
\frac{\left[b_{04}^{5}|\eta|-\left(D_{0} \psi\right)^{2}\right]}{|\eta|} Y_{2}^{4}+b_{01}^{5} Y_{2}^{1}-\frac{2 D_{0} \psi}{|\eta|} D_{0} Y_{1}^{4}-\left[D_{*} \psi b_{01}^{5}+D_{0} \psi b_{01}^{4}+\left(D b_{01}^{5}\right)_{*}(\psi)\right]
$$




$$
\begin{aligned}
& +\left[-D_{0}^{2} \psi+2\left(D_{0} \psi\right)^{2} D_{*} \psi-D_{*} \psi b_{04}^{5}+\left(D b_{04}^{5}\right)_{*}(\psi)\right] Y_{1}^{4} \\
& +2 D_{0} \psi D_{*} \psi D_{0} Y_{0}^{4}+\left(D_{0} \psi\right)^{2} D_{*} Y_{0}^{4}-D_{0}^{2} Y_{0}^{4}-b_{01}^{4} D_{0} Y_{0}^{1} \\
& +\left[2 D_{0} \psi D_{*} \psi b_{01}^{4}-D_{0} b_{01}^{4}-D_{*} b_{01}^{5}+\left(D_{*} \psi\right)^{2} b_{01}^{5}+b_{11}^{5}+\sum \partial^{j k} b_{01}^{5} \partial_{j} \psi \partial_{k} \psi\right] Y_{0}^{1} \\
& +\left(D b_{01}^{5}\right)_{*}(\psi) Y_{0}^{1}+\left[-3\left(D_{0} \psi\right)^{2}\left(D_{*} \psi\right)^{2}+3 D_{0} \psi D_{* 0}^{2} \psi+2 D_{*} \psi D_{0}^{2} \psi\right. \\
& \left.\quad-D_{*} b_{04}^{5}+\left(D_{*} \psi\right)^{2} b_{04}^{5}+b_{14}^{5}+\sum \partial^{j k} b_{04}^{5} \partial_{j} \psi \partial_{k} \psi \psi\right] Y_{0}^{4}=0 .
\end{aligned}
$$

Pour les $Z$ on a, comme précédemment

$$
\begin{aligned}
& Z_{0}^{2}=Z_{0}^{3}=Z_{0}^{5}=Z_{1}^{2}=Z_{1}^{3}=Z_{1}^{5}=0 \\
& |\eta| Z_{2}^{2}+D_{0} Z_{0}^{1}=0 ; \quad|\eta| Z_{2}^{5}+D_{0} Z_{0}^{4}+b_{01}^{4} Z_{0}^{1}=0 \\
& Z_{2}^{3}=0, \quad b_{01}^{5} Z_{0}^{1}+b_{04}^{5} Z_{0}^{4}=0
\end{aligned}
$$

à l'étape (4) à la $3^{\text {ème }}$ ligne:

$$
\left(b_{02}^{3} b_{04}^{5}-b_{05}^{3} b_{01}^{5}\right) D_{0} Z_{0}^{4}+W Z_{0}^{4}=0 ;
$$

il reste à déterminer $Z_{0}^{4} \mid$.

Les conditions initiales imposées sont les mêmes que dans le cas précédent et on a

$$
\begin{aligned}
& \sum_{t} Y_{0}^{t 4}\left|+Z_{0}^{4}\right|=0 ; \quad \sum_{t}\left(D_{0} \psi^{t}\right)^{2} Y_{0}^{t 4}\left|=0 ; \quad \sum_{t} D_{0} \psi^{t} Y_{0}^{t 4}\right|=0 \\
& \sum_{t}\left(D_{0} \psi^{t}\right)^{3} Y_{0}^{t 4}\left|=0 ; \quad \sum_{t}\left(D_{0} \psi^{t}\right)^{4} Y_{0}^{t 4}\right|=b_{01}^{5} \mid \eta^{3} I^{3}
\end{aligned}
$$

on en déduit de la dernière équation

$$
\left(b_{02}^{3} b_{04}^{5}-b_{05}^{3} b_{01}^{5}\right) \sum_{t} Y_{0}^{t 4}\left|=b_{01}^{5}\right| \eta \mid I^{3}
$$

on obtient donc les $Y_{0}^{t 4} \mid$ et $Z_{0}^{4} \mid$ et tous les coefficients.

a) ${ }_{2} b_{04}^{3}=b_{01}^{5}=0, b_{02}^{3} b_{04}^{5} \neq 0, b_{02}^{3} \neq b_{04}^{5}$.

$$
\begin{aligned}
f & =\sum_{1 \leq t \leq 2} f_{11}^{t}+\sum_{1 \leq t^{\prime} \leq 2} f_{12}^{t^{\prime}}+f_{2} ; \\
f_{11}^{t} & =e^{\psi_{1}^{t}}\left[\left(Y_{1}^{t}\right)_{0}+\cdots+\left(Y_{1}^{t}\right)_{k}+\cdots\right],
\end{aligned}
$$

$\left(D_{0} \psi_{1}^{t}\right)^{2}=b_{02}^{3}|\eta| ; \psi_{1}^{t}$ est d'ordre $1 / 2 ;\left(Y_{1}\right)_{k}^{t}$ d'ordre $\frac{2-k}{2}$.

$$
f_{12}^{t^{\prime}}=e^{\psi_{2}^{t^{\prime}}}\left[\left(Y_{2}\right)_{0}^{t^{\prime}}+\cdots+\left(Y_{2}\right)_{k}^{t^{\prime}}+\cdots\right]
$$


$\left(D_{0} \psi_{2}^{t^{\prime}}\right)^{2}=b_{04}^{5}|\eta| ; \psi_{2}^{t^{\prime}}$ est d'ordre $1 / 2 ;\left(Y_{2}\right)_{k}^{t^{\prime}}$ d'ordre $\frac{1-k}{2}$.

$$
f_{2}=Z_{0}+\cdots+Z_{k}+\cdots ;
$$

$Z_{k}$ est d'ordre $\frac{2-k}{2}$.

On obtient pour les $Y_{1}^{t}$ :

$$
\left(Y_{1}\right)_{0}^{2}=\left(Y_{1}\right)_{0}^{3}=\left(Y_{1}\right)_{0}^{5}=0 ; \quad|\eta|\left(Y_{1}\right)_{1}^{2}+D_{0} \psi_{1}\left(Y_{1}\right)_{0}^{1}=0, \quad\left(Y_{1}\right)_{1}^{3}=0 ; \quad\left(Y_{1}\right)_{0}^{4}=0
$$

d'où:

$$
\begin{gathered}
\left(Y_{1}\right)_{1}^{5}=0, \quad|\eta|\left(Y_{1}\right)_{2}^{2}+\cdots+D_{0}\left(Y_{1}\right)_{0}^{1}+W\left(Y_{1}\right)_{0}^{1}=0 \\
|\eta|\left(Y_{1}\right)_{2}^{3}-\frac{\left(D_{0} \psi_{1}\right)^{2}}{|\eta|}\left(Y_{1}\right)_{0}^{1}=0, \quad|\eta|\left(Y_{1}\right)_{2}^{5}+D_{0} \psi_{1}\left(Y_{1}\right)_{1}^{4}+b_{01}^{4}\left(Y_{1}\right)_{0}^{1}=0
\end{gathered}
$$

la $3^{\text {ème }}$ ligne de la $3^{\text {ème }}$ étape donne:

$$
\left[\frac{\left(D_{0} \psi_{1}\right)^{3}}{|\eta|^{2}}-\frac{b_{02}^{3} D_{0} \psi_{1}}{|\eta|}\right]\left(Y_{1}\right)_{0}^{1}=0
$$

$\left(Y_{1}\right)_{0}^{1}$ reste libre; la $5^{\text {ème }}$ ligne donne:

$$
\begin{aligned}
& \left(b_{04}^{5}-b_{02}^{3}\right)\left(Y_{1}\right)_{1}^{4}-\frac{D_{0} \psi_{1} b_{01}^{4}}{|\eta|}\left(Y_{1}\right)_{0}^{1}=0, \\
& |\eta|\left(Y_{1}\right)_{3}^{3}+\cdots-\frac{2 D_{0} \psi_{1}}{|\eta|} D_{0}\left(Y_{1}\right)_{0}^{1}+W\left(Y_{1}\right)_{0}^{1}=0
\end{aligned}
$$

à la $4^{\text {ème }}$ étape, la $3^{\text {ème }}$ ligne donne:

$$
\left[3 \frac{\left(D_{0} \psi_{1}\right)^{2}}{|\eta|^{2}}-\frac{b_{02}^{3}}{|\eta|}\right] D_{0}\left(Y_{1}\right)_{0}^{1}+W^{\prime}\left(Y_{1}\right)_{0}^{1}=0
$$

c'est-à-dire:

$$
\frac{2 b_{02}^{3}}{|\eta|} D_{0}\left(Y_{1}\right)_{0}^{1}+W^{\prime}\left(Y_{1}\right)_{0}^{1}=0
$$

il reste à déterminer $\left(Y_{1}\right)_{0}^{1} \mid$.

Pour les $Y_{2}^{t^{\prime}}$, on a:

$$
\begin{aligned}
& \left(Y_{2}\right)_{0}^{2}=\left(Y_{2}\right)_{0}^{3}=\left(Y_{2}\right)_{0}^{5}=0 ; \quad|\eta|\left(Y_{2}\right)_{1}^{2}+\left(D_{0} \psi_{2}\right)\left(Y_{2}\right)_{0}^{1}=0, \quad\left(Y_{2}\right)_{1}^{3}=0 ; \\
& |\eta|\left(Y_{2}\right)_{1}^{5}+D_{0} \psi_{2}\left(Y_{2}\right)_{0}^{4}=0, \quad\left[-\frac{\left(D_{0} \psi_{2}\right)^{2}}{|\eta|}+b_{04}^{5}\right]\left(Y_{2}\right)_{0}^{4}=0,
\end{aligned}
$$

$\left(Y_{2}\right)_{0}^{4}$ reste libre. 


$$
\begin{aligned}
& |\eta|\left(Y_{2}\right)_{2}^{2}+\cdots+D_{0}\left(Y_{2}\right)_{0}^{1}+W\left(Y_{2}\right)_{0}^{1}=0 \\
& |\eta|\left(Y_{2}\right)_{2}^{3}-\frac{\left(D_{0} \psi_{2}\right)^{2}}{|\eta|}\left(Y_{2}\right)_{0}^{1}=0 \\
& |\eta|\left(Y_{2}\right)_{2}^{5}+D_{0} \psi_{2}\left(Y_{2}\right)_{1}^{4}+\left(D_{0} Y_{2}\right)_{0}^{4}+W^{\prime}\left(Y_{2}\right)_{0}^{1}+W^{\prime \prime}\left(Y_{2}\right)_{0}^{4}=0
\end{aligned}
$$

à la $3^{\text {ème }}$ étape, on a, à la $3^{\text {ème }}$ ligne:

$$
\left(b_{04}^{5}-b_{02}^{3}\right)|\eta|\left(Y_{2}\right)_{0}^{1}-b_{05}^{3}|\eta|\left(Y_{2}\right)_{0}^{4}=0,
$$

et à la $5^{\text {ème }}$ ligne:

$$
\frac{-2 D_{0} \psi_{2}}{|\eta|} D_{0}\left(Y_{2}\right)_{0}^{4}+W^{\prime \prime \prime}\left(Y_{2}\right)_{0}^{4}=0
$$

il reste à déterminer $\left(Y_{2}\right)_{0}^{4} \mid$.

Pour les $Z$, on obtient:

$$
\begin{aligned}
& Z_{0}^{2}=Z_{0}^{3}=Z_{0}^{5}=Z_{1}^{2}=Z_{1}^{2}=Z_{1}^{3}=Z_{1}^{5}=0 ; \quad Z_{0}^{4}=0 \\
& |\eta| Z_{2}^{2}+D_{0} Z_{0}^{1}=0, \quad|\eta| Z_{2}^{5}+b_{01}^{4} Z_{0}^{1}=0, \quad Z_{1}^{4}=0, \quad \ldots ; \\
& -b_{02}^{3} D_{0} Z_{0}^{1}+\left[-b_{04}^{3} b_{01}^{4}+b_{11}^{3}|\eta|\right] Z_{0}^{1}=0 ;
\end{aligned}
$$

il reste à déterminer $Z_{0}^{1} \mid$.

On impose les conditions initiales:

on obtient:

$$
\begin{aligned}
& \sum_{t}\left(Y_{1}\right)_{0}^{t}\left|+Z_{0}\right|=0 ; \quad \sum_{t}\left(Y_{2}\right)_{0}^{t^{\prime}}\left|+\sum_{t}\left(Y_{1}\right)_{1}^{t}\right|+Z_{1} \mid=0 \\
& \sum_{t}\left(Y_{2}\right)_{1}^{t^{\prime}}\left|+\sum_{t}\left(Y_{1}\right)_{2}^{t}\right|+Z_{2} \mid=I ; \quad \ldots ;
\end{aligned}
$$

$$
\begin{aligned}
& \sum_{t}\left(Y_{1}\right)_{0}^{t 1}\left|+Z_{0}^{1}\right|=0 ; \quad \sum_{t} D_{0} \psi_{1}\left(Y_{1}\right)_{0}^{t 1}\left|=0 ; \quad \sum_{t}\left(Y_{2}\right)_{0}^{t^{\prime} 4}\right|=0 \\
& b_{02}^{3} \sum_{t}\left(Y_{1}\right)_{0}^{t 1}|=| \eta \mid I^{3} ;
\end{aligned}
$$

on en déduit les $Y_{1}^{t 1} \mid$ et $Z_{0}^{1} \mid$;

$$
-\sum_{t^{\prime}} D_{0} \psi_{2}^{t^{\prime}}\left(Y_{2}\right)_{0}^{t^{\prime} 4}\left|-\frac{b_{02}^{3} b_{01}^{4}}{b_{04}^{5}-b_{02}^{3}} \sum_{t}\left(Y_{1}\right)_{0}^{t 1}\right|=|\eta| I^{5}
$$

on en déduit les $\left(Y_{2}\right)_{0}^{t^{\prime} 4} \mid$ et par suite tous les coefficients. 
1b) et 1c) On a: $\left(b_{02}^{3}+b_{04}^{5}\right)^{2}+4\left(b_{04}^{3} b_{01}^{4}+b_{05}^{3} b_{01}^{5}-b_{02}^{3} b_{04}^{5}\right)=0$.

Précisons les coefficients $C_{1}$ et $C_{2}$ introduits dans 1) a) $)_{1^{\prime}}$ et a) $)_{1^{\prime \prime}}$

$$
\begin{aligned}
|\eta| C_{1}= & \left(\frac{b_{02}^{3}+b_{04}^{5}}{2}\right)\left[b_{11}^{3}|\eta|-b_{04}^{3} D_{0}\left(\frac{b_{02}^{3}}{b_{04}^{3}}\right)+b_{04}^{3} D_{0}\left(\frac{b_{04}^{5}}{b_{04}^{3}}\right)\right] \\
& \quad+b_{05}^{3} b_{04}^{5} b_{01}^{4}-b_{04}^{5} b_{11}^{3}|\eta|-b_{04}^{3} D_{0} b_{01}^{4}+b_{04}^{3} b_{11}^{5}|\eta| \\
C_{2}= & \left.\frac{b_{02}^{3}+b_{04}^{5}}{2}\right)\left[b_{11}^{3}|\eta|-D_{0} b_{02}^{3}-2 D_{0} b_{04}^{5}+2 D_{0}\left(\frac{b_{02}^{3}}{b_{01}^{5}}\right) b_{01}^{5}\right] \\
& +b_{05}^{3} b_{04}^{5} b_{01}^{4}-b_{04}^{5} b_{11}^{3}|\eta|+b_{01}^{5} b_{14}^{3}|\eta|+b_{02}^{3} D_{0} b_{04}^{5}-b_{05}^{3} D_{0} b_{01}^{5} .
\end{aligned}
$$

Notons aussi dans le cas 1a) $)_{2}: C^{\prime}=b_{05}^{3} b_{01}^{4}$.

Notons brièvement $C$ l'un de ces coefficients.

1b) On suppose de plus: $C=0$

b) ${ }_{1} b_{04}^{3}$ ou $b_{01}^{5} \neq 0$.

$$
\begin{aligned}
f & =\sum_{1 \leq t \leq 2} f_{1}^{t}+f_{2} ; \\
f_{1}^{t} & =e^{\psi^{t}}\left[Y_{0}^{t}+\cdots+Y_{k}^{t}+\cdots\right]
\end{aligned}
$$

$\left(D_{0} \psi^{t}\right)^{2}=\frac{b_{02}^{3}+b_{04}^{5}}{2}|\eta| \neq 0 ; \psi^{t}$ est d'ordre $\frac{1}{2} ; Y_{k}^{t}$ est d'ordre $\frac{3-k}{2} ;$

$$
f_{2}=Z_{0}+\cdots+Z_{k}+\cdots
$$

$Z_{k}$ est d'ordre $\frac{2-k}{2}$.

b) $1^{\prime} b_{04}^{3} \neq 0, b_{01}^{5}=0$. On reprend le calcul du 1a) $)_{1^{\prime}}$ que l'on doit pour-

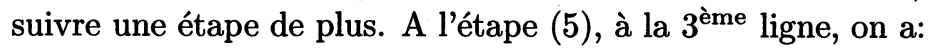

$$
\begin{gathered}
b_{04}^{3} Y_{3}^{4}+\cdots+3 D_{0} \psi \frac{D_{0}^{2} Y_{0}^{1}}{|\eta|^{2}}+\left[\frac{-2\left(D_{0} \psi\right)^{3}+b_{02}^{3} D_{0} \psi|\eta|}{|\eta|^{3}}\right] D_{*} Y_{0}^{1} \\
+W D_{0} Y_{0}+W^{\prime} Y_{0}^{1}-\frac{D_{0} \psi}{|\eta|}\left(D b_{02}^{3}\right)_{*}\left(Y_{0}^{1}\right) \\
+\frac{D_{0} \psi}{b_{04}^{3}|\eta|^{2}}\left[b_{02}^{3}|\eta|-\left(D_{0} \psi\right)^{2}\right]\left(D b_{04}^{3}\right)_{*}\left(Y_{0}^{1}\right)=0
\end{gathered}
$$

à la $5^{\text {ème }}$ ligne, on a:

$$
\begin{aligned}
& {\left[\frac{b_{04}^{5}|\eta|-\left(D_{0} \psi\right)^{2}}{|\eta|}\right] Y_{3}^{4}+\cdots+\frac{D_{0} \psi\left(7\left(D_{0} \psi\right)^{2}-3 b_{02}^{3}|\eta|\right)}{b_{04}^{3}|\eta|^{3}} D_{0}^{2} Y_{0}^{1}} \\
& \quad+\left[\frac{\left(D_{0} \psi\right)^{3}}{b_{04}^{3}|\eta|^{5}}\left(b_{02}^{3}|\eta|-\left(D_{0} \psi\right)^{2}\right)+\frac{D_{0} \psi b_{01}^{4}}{|\eta|}\right] D_{*} Y_{0}^{1} \\
& \quad-\frac{D_{0} \psi}{|\eta|}\left(D b_{01}^{4}\right)_{*}\left(Y_{0}^{1}\right)+\frac{\left(D_{0} \psi\right)}{b_{04}^{3}|\eta|^{2}}\left(b_{02}^{3}|\eta|-\left(D_{0} \psi\right)^{2}\right)\left(D b_{04}^{5}\right)_{*}\left(Y_{0}^{1}\right)=0
\end{aligned}
$$


d'où:

$$
\frac{4\left(D_{0} \psi\right)^{3}}{|\eta|^{3}} D_{0}^{2} Y_{0}^{1}+W D_{0} Y_{0}^{1}+W^{\prime} Y_{0}^{1}=0
$$

pour les $Z$, le calcul est identique à celui de $1 \mathrm{a})_{1^{\prime}}$; il reste à déterminer $Z_{0}^{1} \mid$. On impose les conditions initiales:

$$
\sum_{t} Y_{0}^{t}\left|=0 ; \quad \sum_{t} Y_{1}^{t}\right|+Z_{0}\left|=0 ; \quad \sum_{t} Y_{2}^{t}\right|+Z_{1}\left|=0 ; \quad \sum_{t} Y_{3}^{t}\right|+Z_{2} \mid=I ; \quad \ldots
$$

on obtient:

$$
\sum_{t} Y_{0}^{t 1}\left|=0 ; \quad \sum_{t} Y_{1}^{t 1}\right|+Z_{0}^{1}\left|=0 ; \quad \sum_{t} D_{0} \psi^{t} Y_{0}^{t 1}\right|=0
$$

d'où $Y_{0}^{t 1} \mid=0$; ensuite:

$$
\begin{aligned}
& \sum_{t} D_{0} \psi^{t} Y_{1}^{t 1}\left|+\sum_{t} D_{0} Y_{0}^{t 1}\right|=0 \\
& \left(b_{02}^{3}-b_{04}^{5}\right) \sum_{t} D_{0} \psi^{t} Y_{1}^{t 1}\left|-\left(b_{02}^{3}+3 b_{04}^{5}\right) \sum_{t} D_{0} Y_{0}^{t 1}\right|=0
\end{aligned}
$$

d'où:

$$
\sum_{t} D_{0} Y_{0}^{t 1} \mid=0
$$

enfin, on a:

$$
\begin{aligned}
& \frac{b_{02}^{3}+b_{04}^{5}}{2}|\eta| \sum_{t} Y_{1}^{t 1}\left|+2 \sum_{t} D_{0} \psi^{t} Y_{0}^{t 1}\right|=|\eta|^{2} I^{3} \\
& \frac{\left(b_{02}^{3}+b_{04}^{5}\right)\left(b_{04}^{5}-b_{02}^{3}\right)}{4 b_{04}^{3}}|\eta| \sum_{t} Y_{1}^{t 1}\left|+\frac{2 b_{04}^{5} \mid}{b_{04}^{3} \mid} \sum_{t} D_{0} \psi^{t} D_{0} Y_{0}^{t 1}\right|=|\eta|^{2} I^{5}
\end{aligned}
$$

on en déduit les valeurs de $\sum_{t} Y_{1}^{t 1} \mid$ et de $\sum_{t} D_{0} \psi^{t} D_{0} Y_{0}^{t 1} \mid$, d'où les $D_{0} Y_{0}^{t 1} \mid$ et $Z_{0}^{1} \mid$.

b) ${ }_{1^{\prime \prime}} b_{01}^{5} \neq 0, b_{04}^{3}=0$. Le calcul est analogue; on obtient:

$$
\begin{aligned}
& \frac{4\left(D_{0} \psi\right)^{3}}{|\eta|^{3}} D_{0}^{2} Y_{0}^{4}+W D_{0} Y_{0}^{4}+W^{\prime} Y_{0}^{4}=0 \\
& \left(b_{02}^{3} b_{04}^{5}-b_{05}^{3} b_{01}^{5}\right) D_{0} Z_{0}^{4}+W^{\prime \prime} Z_{0}^{4}=0
\end{aligned}
$$

on impose les mêmes conditions aux $Y \mid$ et $Z \mid$ que dans le cas précédent et on détermine tous les coefficients. 
b) ${ }_{2} b_{04}^{3}=b_{01}^{5}=0 ; b_{02}^{3}=b_{04}^{5} ; b_{02}^{3} \neq 0, b_{05}^{3} b_{01}^{4}=0$.

$$
\begin{aligned}
f & =\sum_{1 \leq t \leq 2} f_{1}^{t}+f_{2} ; \\
f_{1}^{t} & =e^{\psi^{t}}\left[Y_{0}^{t}+\cdots+Y_{k}^{t}+\cdots\right]
\end{aligned}
$$

$\left(D_{0} \psi^{t}\right)^{2}=b_{02}^{3}|\eta| ; \psi^{t}$ est d'ordre $1 / 2 ; Y_{k}^{t}$ est d'ordre $\frac{2-k}{2}$;

$$
f_{2}=Z_{0}+\cdots+Z_{k}+\cdots
$$

$Z_{k}$ est d'ordre $\frac{2-k}{2}$

b) $2_{2^{\prime}} b_{05}^{3} \neq 0, b_{01}^{4}=0$. On a:

$$
Y_{0}^{2}=Y_{0}^{3}=Y_{0}^{5}=0 ; \quad \ldots ; \quad Y_{0}^{4}=0 ;
$$

à la $4^{\text {ème }}$ étape, à la $3^{\text {ème }}$ et à la $5^{\text {ème }}$ lignes, on a:

$$
\begin{aligned}
& 2 b_{02}^{3} D_{0} Y_{0}^{1}-b_{05}^{3} D_{0} \psi Y_{1}^{4}+\left(b_{11}^{3}+D_{0} b_{2}^{3}\right) Y_{0}^{1}=0 \\
& 2 D_{0} \psi D_{0} Y_{1}^{4}+W Y_{1}^{4}+W^{\prime} Y_{0}^{1}=0
\end{aligned}
$$

d'où

$$
\begin{aligned}
& \frac{4 b_{02}^{3}}{b_{05}^{3}} D_{0}^{2} Y_{0}^{1}+W^{\prime \prime} D_{0} Y_{0}^{1}+W^{\prime \prime \prime} Y_{0}^{1}=0 \\
& Z_{0}^{2}=Z_{0}^{3}=Z_{0}^{5}=0 ; \quad Z_{0}^{4}=0 ; \quad Z_{1}^{2}=Z_{1}^{3}=Z_{1}^{5}=0 ; \quad Z_{1}^{4}=0 \\
& -b_{02}^{3} D_{0} Z_{0}^{1}+b_{11}^{3}|\eta| Z_{0}^{1}=0
\end{aligned}
$$

On impose:

on a:

$$
\sum_{t} Y_{0}^{t}\left|+Z_{0}\right|=0 ; \quad \sum_{t} Y_{1}^{t}\left|+Z_{1}\right|=0 ; \quad \sum_{t} Y_{2}^{t}\left|+Z_{2}\right|=I ; \quad \cdots
$$

$$
\begin{aligned}
& \sum_{t} Y_{0}^{t 1}\left|+Z_{0}^{1}\right|=0 ; \quad \sum_{t} D_{0} \psi^{t} Y_{0}^{t 1}\left|=0, \quad \sum_{t} D_{0} \psi^{t} D_{0} Y_{0}^{t 1}\right|=0 \\
& b_{02}^{3} \sum_{t} Y_{0}^{t 1}|=| \eta \mid I^{3}
\end{aligned}
$$

on en déduit les $Y_{0}^{t 1} \mid$ et $Z_{0}^{1} \mid$; enfin:

$$
\frac{2 b_{02}^{3}}{b_{05}^{3}} \sum_{t} D_{0} Y_{0}^{t 1} \mid+ \text { termes connus }=I^{5}
$$

d'où l'on en déduit $D_{0} Y_{0}^{t 1} \mid$ et tous les coefficients. 
b) $2^{\prime \prime} b_{05}^{3}=0 . \quad$ On a:

$$
\begin{aligned}
& Y_{0}^{2}=Y_{0}^{3}=Y_{0}^{5}=0 \\
& 2 D_{0} \psi D_{0} Y_{0}^{4}+W Y_{0}^{1}+W^{\prime} Y_{0}^{4}=0 \\
& 2 b_{02}^{3} D_{0} Y_{0}^{1}+W^{\prime \prime} Y_{0}^{1}+W^{\prime \prime \prime} Y_{0}^{4}=0
\end{aligned}
$$

il reste à déterminer $Y_{0}^{1} \mid$ et $Y_{0}^{4} \mid$; on a encore

$$
b_{02}^{3} D_{0} Z_{0}^{1}+W^{I V} Z_{0}^{1}=0
$$

il reste $Z_{0}^{1} \mid$ à déterminer; on impose les mêmes conditions que dans b) ${ }_{1}$ et on obtient:

$$
\sum_{t} Y_{0}^{t 1}\left|+Z_{0}^{1}\right|=0, \quad Y_{0}^{4}\left|=0 ; \quad \sum_{t} D_{0} \psi^{t} Y_{0}^{t 1}\right|=0 ; \quad b_{02}^{3} \sum_{t} Y_{0}^{t 1}|=| \eta \mid I^{3}
$$

d'où les $Y_{0}^{t 1} \mid$ et $Z_{0}^{1} \mid$; on détermine tous les coefficients $Y$ et $Z$.

1c) $C \neq 0$.

c) ${ }_{1} b_{04}^{3}$ ou $b_{01}^{5} \neq 0$.

$$
f=\sum_{\substack{1 \leq t \leq 2 \\ 1 \leq t^{\prime} \leq 2}} f_{1}^{t t^{\prime}}+f_{2}
$$

avec

$$
f_{1}^{t t^{\prime}}=e^{\psi_{1}^{t}+\psi_{2}^{t t^{\prime}}}\left[Y_{0}^{t t^{\prime}}+\cdots+Y_{k}^{t t^{\prime}}+\cdots\right]
$$

$2\left(D_{0} \psi_{1}^{t}\right)^{2}=\left(b_{02}^{3}+b_{04}^{5}\right)|\eta| ; \psi_{1}^{t}$ est d'ordre $1 / 2 ;\left(D_{0} \psi_{2}^{t t^{\prime}}\right)^{2}=-\frac{D_{0} \psi_{1}^{t} C}{2\left(b_{02}^{3}+b_{04}^{5}\right)} ; \psi_{2}^{t t^{\prime}}$ est d'ordre $1 / 4 ; Y_{k}^{t t^{\prime}}$ est d'ordre: $\frac{5-k}{4}$;

$$
f_{2}=Z_{0}+\cdots+Z_{k}+\cdots
$$

$Z_{k}$ est d'ordre: $\frac{4-k}{4}$.

Le système différentiel des $Y$ est le même que celui du $§ 1.51 \mathrm{c})$.

c) ${ }_{1} b_{04}^{3} \neq 0$. En bref, on a:

$$
Y_{0}^{2}=Y_{0}^{3}=Y_{0}^{4}=Y_{0}^{5}=0
$$

à la $6^{\text {ème }}$ étape, on obtient:

$$
\left[\left(D_{0} \psi_{1}\right)^{4}-\left(b_{02}^{3}+b_{04}^{5}\right)\left(D_{0} \psi_{1}\right)^{2}|\eta|+\left(b_{02}^{3} b_{04}^{5}-b_{04}^{3} b_{05}^{4}\right)|\eta|^{2}\right] Y_{0}^{1}=0
$$

qui est identiquement vérifiée; à la $7^{\text {ème }}$ étape, on obtient:

$$
\left[2\left(D_{0} \psi_{1}\right)^{4}-\left(D_{0} \psi_{1}\right)^{2}\left(b_{02}^{3}+b_{04}^{5}\right)|\eta|\right] Y_{0}^{1}=0
$$


qui est aussi identiquement vérifiée; à la $8^{\text {ème }}$ étape, on obtient

$$
\left[4 D_{0} \psi_{1}\left(D_{0} \psi_{2}\right)^{2}+C|\eta|\right] Y_{0}^{1}=0
$$

qui est aussi identiquement vérifiée, par le choix de $\psi_{2}$; à la $9^{\text {ème }}$ étape, on a

$$
8\left(D_{0} \psi_{1}\right)^{3} D_{0} \psi_{2} D_{0} Y_{0}^{1}+W Y_{0}^{1}=0 ;
$$

il reste à déterminer $Y_{0}^{1} \mid$.

Le système différentiel en $Z$ est le même que celui de $§ 1.51 \mathrm{c})$. En bref, on a:

$$
Z_{0}^{2}=Z_{0}^{3}=Z_{0}^{4}=Z_{0}^{5}=0
$$

à la $8^{\text {ème }}$ étape, on obtient:

$$
\begin{aligned}
& b_{04}^{3} Z_{4}^{4}-\frac{b_{02}^{3} D_{0} Z_{0}^{1}}{|\eta|}+\left[-\frac{b_{05}^{3} b_{01}^{4}}{|\eta|}+b_{11}^{3}\right] Z_{0}^{1}=0 \\
& b_{04}^{5} Z_{4}^{4}-\frac{b_{01}^{4} D_{0} Z_{0}^{1}}{|\eta|}+\left[-\frac{D_{0} b_{01}^{4}}{|\eta|}+b_{11}^{5}\right] Z_{0}^{1}=0
\end{aligned}
$$

d'où:

$$
\left(b_{04}^{5} b_{02}^{3}-b_{04}^{3} b_{01}^{4}\right) D_{0} Z_{0}^{1}+\left(b_{05}^{3} b_{01}^{4} b_{04}^{5}-b_{04}^{5} b_{11}^{3}+b_{04}^{3} b_{11}^{5}-b_{04}^{3} D_{0} b_{01}^{4}\right) Z_{0}^{1}=0 ;
$$

il reste à déterminer $Z_{0}^{1} \mid$. On impose les conditions initiales:
(0) $\sum_{t t^{\prime}} Y_{0}^{t t^{\prime}} \mid=0$;
(1) $\sum_{t t^{\prime}} Y_{1}^{t t^{\prime}}\left|+Z_{0}\right|=0$
(2) $\sum_{t t^{\prime}} Y_{2}^{t t^{\prime}}\left|+Z_{1}\right|=0$
(3) $\sum_{t t^{\prime}} Y_{3}^{t t^{\prime}}\left|+Z_{2}\right|=0$;
(4) $\sum_{t t^{\prime}} Y_{4}^{t t^{\prime}}\left|+Z_{3}\right|=0$
(5) $\sum_{t t^{\prime}} Y_{5}^{t t^{\prime}}\left|+Z_{4}\right|=I ; \ldots$;

on en déduit:
(0) $\quad \sum_{t t^{\prime}} Y_{0}^{t t^{\prime} 1} \mid=0$
(1) $\sum_{t t^{\prime}} Y_{1}^{t t^{\prime} 1}\left|+Z_{0}^{1}\right|=0$;
(2) $\quad \sum_{t t^{\prime}} D_{0} \psi_{1}^{t} Y_{0}^{t t^{\prime} 1} \mid=0$;
(3) $\sum_{t t^{\prime}} D_{0} \psi_{2}^{t t^{\prime}} Y_{0}^{t t^{\prime} 1} \mid=0$;
(5) $\left.\frac{b_{02}^{3}+b_{04}^{5}}{2|\eta|} \sum_{t t^{\prime}} Y_{1}^{t t^{\prime} 1}\left|+\frac{2}{|\eta|^{2}} \sum_{t t^{\prime}} D_{0} \psi_{1}^{t} D_{0} \psi_{2}^{t t^{\prime}} Y_{0}^{t t^{\prime}}\right| \eta\right|^{2} \mid=I^{3}$

$$
\frac{\left(b_{02}^{3}+b_{04}^{5}\right)}{2 b_{04}^{3}|\eta|} \frac{\left(b_{04}^{5}-b_{02}^{3}\right)}{2} \sum_{t t^{\prime}} Y_{1}^{t t^{\prime}}\left|+\frac{2 b_{04}^{5}}{b_{04}^{3}|\eta|^{2}} \sum_{t t^{\prime}} D_{0} \psi_{1}^{t} D_{0} \psi_{2}^{t t^{\prime}} Y_{1}^{t t^{\prime} 1}\right|=I^{5}
$$

on obtient, comme $b_{02}^{3}+b_{04}^{5} \neq 0$, les valeurs de: $\sum_{t t^{\prime}} D_{0} \psi_{1}^{t} D_{0} \psi_{2}^{t t^{\prime}} Y_{0}^{t t^{\prime} 1} \mid$ et de $\sum_{t t^{\prime}} Y_{1}^{t t^{\prime} 1} \mid$; on en déduit comme dans le cas du S1.5 1c) les valeurs initiales de $Y_{0}^{t t^{\prime} 1}$ et de $Z_{0}^{1}$, d'où tous les coefficients. 
c) $1^{\prime \prime} b_{01}^{5} \neq 0$

$$
Y_{0}^{2}=Y_{0}^{3}=Y_{0}^{5}=0 ; \quad b_{01}^{5} Y_{0}^{1}+\left[b_{04}^{5}-\frac{\left(D_{0} \psi_{1}\right)^{2}}{|\eta|}\right] Y_{0}^{4}=0
$$

on exprime $Y_{0}^{1}$ à l'aide de $Y_{0}^{4} ;$ à la $6^{\text {ème }}$ étape, on a:

$$
\left[\left(D_{0} \psi_{1}\right)^{4}-\left(b_{02}^{3}+b_{04}^{5}\right)\left(D_{0} \psi_{1}\right)^{2}|\eta|+\left(b_{02}^{3} b_{04}^{5}-b_{04}^{3} b_{01}^{4}\right)|\eta|^{2}\right] Y_{0}^{4}=0
$$

qui est identiquement vérifiée; la $7^{\text {ème }}$ étape est analogue à celle du cas $\left.\mathrm{c}\right)_{1^{\prime}} ;$ la $8^{\text {ème }}$ étape détermine $D_{0} \psi_{2}$ comme précédemment; la $9^{\text {ème }}$ étape donne l'équation:

$$
D_{0} Y_{0}^{4}+W Y_{0}^{4}=0
$$

comme précédemment.

Pour les $Z$, on a:

$$
b_{01}^{5} Z_{0}^{1}+b_{04}^{5} Z_{0}^{4}=0
$$

on exprime $Z_{0}^{1}$ à l'aide $Z_{0}^{4}$, et on obtient:

$$
\left(b_{02}^{3} b_{04}^{5}-b_{05}^{3} b_{01}^{5}\right) D_{0} Z_{0}^{4}+W^{\prime} Z_{0}^{4}=0
$$

on impose les conditions initiales du cas c) $)_{1^{\prime}}$ et par des calculs du même genre, on obtient les données initiales.

c) ${ }_{2} b_{04}^{3}=b_{01}^{5}=0, b_{02}^{3}=b_{04}^{5} \neq 0, b_{05}^{3} b_{01}^{4} \neq 0$.

$$
f=e^{\psi_{1}^{t}+\psi_{2}^{t t^{\prime}}}\left[Y_{0}^{t t^{\prime}}+\cdots+Y_{k}^{t t^{\prime}}+\cdots\right]
$$

$\left(D_{0} \psi_{1}^{t}\right)^{2}=b_{02}^{3}|\eta| ; \psi_{1}^{t}$ est d'ordre $1 / 2 ;\left(D_{0} \psi_{2}^{t t^{\prime}}\right)^{2}=-\frac{b_{05}^{3} b_{01}^{4}}{4\left(b_{02}^{3}\right)^{2}} D_{0} \psi_{1}^{t} ; \psi_{2}^{t t^{\prime}}$ est d'ordre $1 / 4 ; Y_{k}^{t t^{\prime}}$ est d'ordre: $\frac{4-k}{4}$.

$$
f_{2}=Z_{0}+\cdots+Z_{k}+\cdots
$$

$Z_{k}$ est d'ordre: $\frac{4-k}{4}$.

Le système différentiel en $Y$ est celui des cas a); on obtient;

$$
Y_{0}^{2}=Y_{0}^{3}=Y_{0}^{5}=0
$$

à la $4^{\text {ème }}$ étape:

$$
\left[\left(D_{0} \psi_{1}\right)^{2}-b_{02}^{3}|\eta|\right] Y_{0}^{4}=0
$$


laisse $Y_{0}^{4}$ libre; à la $5^{\text {ème }}$ étape, on a:

$$
Y_{0}^{4}=0
$$

$Y_{0}^{1}$ reste libre; à la $6^{\text {ème }}$ étape, la $5^{\text {ème }}$ ligne donne:

$$
2 D_{0} \psi_{2} Y_{1}^{4}+b_{01}^{4} Y_{0}^{1}=0
$$

à la $7^{\text {ème }}$ étape, la $3^{\text {ème }}$ ligne donne:

$$
-b_{05}^{3} Y_{1}^{4}+\frac{2 D_{0} \psi_{2} D_{0} \psi_{1}}{|\eta|} Y_{0}^{1}=0
$$

on en déduit:

$$
\left[4\left(D_{0} \psi_{2}\right)^{2} D_{0} \psi_{1} b_{02}^{3}+b_{05}^{3} b_{01}^{4}|\eta|\right] Y_{0}^{1}=0
$$

qui laisse $Y_{0}^{1}$ libre; à la $8^{\text {ème }}$ étape, on a:

$$
8\left(b_{02}^{3}\right)^{2}\left(D_{0} \psi_{2}\right)^{2} D_{0} Y_{0}^{1}+W Y_{0}^{1}=0 ;
$$

pour les $Z$, on obtient:

$$
Z_{0}^{2}=Z_{0}^{3}=Z_{0}^{4}=Z_{0}^{5}=0 ; \quad-b_{02}^{3} D_{0} Z_{0}^{1}+\left(b_{11}^{3}|\eta|-b_{05}^{3} b_{01}^{4}\right) Z_{0}^{1}=0 .
$$

On impose:

$$
\begin{array}{lll}
\sum_{t t^{\prime}} Y_{0}^{t t^{\prime}}\left|+Z_{0}\right|=0 ; & \sum_{t t^{\prime}} Y_{1}^{t t^{\prime}}+Z_{1} \mid=0 ; & \sum_{t t^{\prime}} Y_{2}^{t t^{\prime}}\left|+Z_{2}\right|=0 \\
\sum_{t t^{\prime}} Y_{3}^{t t^{\prime}}\left|+Z_{3}\right|=0 ; & \sum_{t t^{\prime}} Y_{4}^{t t^{\prime}}\left|+Z_{4}\right|=I ; & \ldots ;
\end{array}
$$

on obtient:
(0) $\sum_{t t^{\prime}} Y_{0}^{t t^{\prime}}\left|+Z_{0}^{1}\right|=0$;
(1) $\quad \sum_{t t^{\prime}} D_{0} \psi_{1}^{t} D_{0} \psi_{2}^{t t^{\prime}} Y_{0}^{t t^{\prime} 1} \mid=0$;
(2) $\sum_{t t^{\prime}} D_{0} \psi_{1}^{t} Y_{0}^{t t^{\prime} 1} \mid=0$;
(3) $\sum_{t t^{\prime}} D_{0} \psi_{2}^{t t^{\prime}} Y_{0}^{t t^{\prime} 1} \mid=0$;
(4) $\quad b_{02}^{3} \sum_{t t^{\prime}} Y_{0}^{t t^{\prime} 1}\left|=I^{3}\right| \eta \mid$

on obtient comme précédemment tous les coefficients.

2) $b_{04}^{3} b_{01}^{4}+b_{05}^{3} b_{01}^{5}-b_{02}^{3} b_{04}^{5}=0 ; b_{02}^{3}+b_{04}^{5} \neq 0$. On distingue les cas

2a) $\quad N$ est formé des points $0,(2,0),(5,2)$. 
2a) ${ }_{1^{\prime}} b_{04}^{3} \neq 0, C^{\prime}=b_{05}^{3} b_{04}^{5} b_{01}^{4}-b_{04}^{3} D_{0} b_{01}^{4}+\left(b_{04}^{3} b_{11}^{5}-b_{11}^{3} b_{04}^{5}\right)|\eta| \neq 0$.

2a) ${ }_{1^{\prime \prime}} b_{01}^{5} \neq 0, C^{\prime \prime}=b_{05}^{3} b_{04}^{5} b_{01}^{4}+b_{02}^{3} b_{01}^{5} D_{0}\left(\frac{b_{04}^{5}}{b_{01}^{5}}\right)-b_{11}^{3} b_{04}^{5}|\eta| \neq 0$.

$$
\begin{aligned}
f & =\sum_{1 \leq t \leq 2} f_{1}^{t}+\sum_{1 \leq t^{\prime} \leq 3} f_{2}^{t^{\prime}} \\
f_{1}^{t} & =e^{\psi^{t}}\left[Y_{0}^{t}+\cdots+Y_{k}^{t}+\cdots\right]
\end{aligned}
$$

$\left(D_{0} \psi^{t}\right)^{2}=\left(b_{02}^{3}+b_{04}^{5}\right)|\eta| ; \psi^{t}$ est d'ordre $1 / 2 ; Y_{k}^{t}$ est d'ordre $\frac{6-k}{6} ;$

$$
f_{2}^{t^{\prime}}=e^{\chi^{t^{\prime}}}\left[Z_{0}^{t^{\prime}}+\cdots+Z_{k}^{t^{\prime}}+\cdots\right]
$$

$\left(D_{0} \chi^{t^{\prime}}\right)^{3}=\frac{C|\eta|}{\left(b_{02}^{3}+b_{04}^{5}\right)}$, où $C=C^{\prime}$ ou $C^{\prime \prime} ; \chi^{t^{\prime}}$ est d'ordre $1 / 3 ; Z_{k}^{t^{\prime}}$ est d'ordre $\frac{8-k}{6}$.

Les systèmes différentiels en $Y$ et $Z$ sont les mêmes que dans le $\S 1.52 \mathrm{a}$ ). On a en bref:

a) $1^{\prime}$

$$
Y_{0}^{2}=Y_{0}^{3}=Y_{0}^{5}=0 ; \quad \ldots ; \quad Y_{0}^{4}=0 ; \quad \ldots ;
$$

à la 9 ème étape:

$$
\left(D_{0} \psi\right)^{2}\left[\left(D_{0} \psi\right)^{2}-\left(b_{02}^{3}+b_{04}^{5}\right)|\eta|\right] Y_{0}^{1}=0
$$

laisse $Y_{0}^{1}$ libre; à la $12^{\text {ème }}$ étape, on a l'équation de propagation:

$$
2\left(D_{0} \psi_{1}\right)^{2}\left(b_{02}^{3}+b_{04}^{5}\right) D_{0} Y_{0}^{1}+W Y_{0}^{1}=0
$$

Pour les $Z$, on a:

$$
Z_{0}^{2}=Z_{0}^{3}=Z_{0}^{5}=0 ; \quad \cdots ; \quad Z_{0}^{4}=0 ;
$$

à la $10^{\text {ème }}$ étape:

$$
\left(b_{02}^{3} b_{04}^{5}-b_{04}^{3} b_{01}^{4}\right) D_{0} \chi Z_{0}^{1}=0
$$

elle laisse $Z_{0}^{1}$ libre; à la $12^{\text {ème }}$ étape:

$$
\left[-\left(D_{0} \chi\right)^{3}\left(b_{02}^{3}+b_{04}^{5}\right)+C|\eta|\right] Y_{0}^{1}=0
$$

$Y_{0}^{1}$ reste libre; à la $14^{\text {ème }}$ étape, on a l'équation de propagation:

$$
3\left(b_{02}^{3}+b_{04}^{5}\right)\left(D_{0} \chi\right)^{2} D_{0} Z_{0}^{1}+W Z_{0}^{1}=0 .
$$


On impose les conditions initiales:

$$
\begin{aligned}
& \sum_{t^{\prime}} Z_{0}^{t^{\prime}}\left|=0 ; \quad \sum_{t^{\prime}} Z_{1}^{t^{\prime}}\right|=0 ; \quad \sum_{t} Y_{0}^{t}\left|+\sum_{t^{\prime}} Z_{2}^{t^{\prime}}\right|=0 ; \ldots \\
& \sum_{t} Y_{5}^{t}\left|+\sum_{t^{\prime}} Z_{7}^{t^{\prime}}\right|=0 ; \quad \sum_{t} Y_{6}^{t}\left|+\sum_{t^{\prime}} Z_{8}^{t^{\prime}}\right|=I ; \quad \ldots ;
\end{aligned}
$$

on en déduit:

$$
\begin{aligned}
& \sum_{t^{\prime}} Z_{0}^{t^{\prime} 1}\left|=0 ; \quad \ldots ; \quad \sum_{t^{\prime}} D_{0} \chi^{t^{\prime}} Z_{0}^{t^{\prime} 1}\right|=0 ; \quad \sum_{t} D_{0} \psi^{t} Y_{0}^{t 1} \mid=0 ; \quad \ldots ; \\
& \left(b_{02}^{3}+b_{04}^{5}\right)|\eta| \sum_{t} Y_{0}^{t 1}\left|+\sum_{t^{\prime}}\left(D_{0} \chi^{t^{\prime}}\right)^{2} Z_{0}^{t^{\prime} 1}\right|=|\eta|^{2} I^{3} \\
& \frac{b_{04}^{5}}{b_{04}^{3}}\left(b_{02}^{3}+b_{04}^{5}\right)|\eta| \sum_{t} Y_{0}^{t 1}\left|-\frac{b_{02}^{3}}{b_{04}^{3}} \sum_{t^{\prime}}\left(D_{0} \chi^{t^{\prime}}\right)^{2} Z_{0}^{t^{\prime} 1}\right|=|\eta|^{2} I^{5}
\end{aligned}
$$

on en déduit $Y_{0}^{t 1} \mid$ et $Z_{0}^{t^{\prime}}{ }^{1} \mid$ et tous les coefficients.

a) ${ }_{1 \prime}$ On détermine les équations de propagation de $Y_{0}^{4}$ et $Z_{0}^{4}$, comme précédemment; on impose les mêmes conditions aux restrictions que dans le cas a $)_{1}$ et on obtient tous les coefficients.

2a) $)_{2} b_{04}^{3}=b_{01}^{5}=0 ; b_{02}^{3}=0, b_{04}^{5} \neq 0 ; b_{05}^{3} b_{01}^{4}-b_{11}^{3}|\eta| \neq 0$.

$$
\begin{aligned}
f & =\sum_{1 \leq t \leq 2} f_{1}^{t}+\sum_{1 \leq t^{\prime} \leq 3} f_{2}^{t^{\prime}} ; \\
f_{1}^{t} & =e^{\psi^{t}}\left[Y_{0}^{t}+\cdots+Y_{k}^{t}+\cdots\right] ;
\end{aligned}
$$

$\left(D_{0} \psi^{t}\right)^{2}=b_{04}^{5}|\eta| ; \psi^{t}$ est d'ordre $1 / 2 ; Y_{k}^{t}$ est d'ordre: $\frac{3-k}{6}$;

$$
f_{2}^{t^{\prime}}=e^{\chi^{t^{\prime}}}\left[Z_{0}^{t^{\prime}}+\cdots+Z_{k}^{t^{\prime}}+\cdots\right]
$$

$\left(D_{0} \chi^{t^{\prime}}\right)^{3}=\left(b_{05}^{3} b_{01}^{4}-b_{11}^{3}|\eta|\right)|\eta| ; \chi^{t^{\prime}}$ est d'ordre $1 / 3 ; Z_{k}^{t^{\prime}}$ est d'ordre: $\frac{8-k}{6}$.

On reprend le système en $Y$ du cas $\mathrm{a}$ ), on obtient:

$$
Y_{0}^{2}=Y_{0}^{3}=Y_{0}^{5}=0 ; \quad \ldots ;
$$

à la $6^{\text {ème }}$ étape:

$$
\left(b_{04}^{5}|\eta|-\left(D_{0} \psi\right)^{2}\right) Y_{0}^{4}=0
$$

qui laisse $Y_{0}^{4}$ libre: . . ; à la $9^{\text {ème }}$ étape:

$$
Y_{0}^{1}=\frac{b_{05}^{3}}{b_{04}^{5}} Y_{0}^{4} \quad \text { et } \quad 2 D_{0} \psi D_{0} Y_{0}^{4}+W Y_{0}^{4}=0 .
$$


Pour les $Z$, on a:

$$
Z_{0}^{2}=Z_{0}^{3}=Z_{0}^{5}=0 ; \quad \ldots, \quad Z_{0}^{4}=0 ; \quad \ldots
$$

à la $12^{\text {ème }}$ étape:

$$
\left[\left(D_{0} \chi\right)^{3}-\left(b_{05}^{3} b_{01}^{4}+b_{11}^{3}|\eta|\right)|\eta|\right] Z_{0}^{1}=0
$$

$Z_{0}^{1}$ reste libre; à la $14^{\text {ème }}$ étape, on a:

$$
3\left(D_{0} \chi\right)^{2} D_{0} Z_{0}^{1}+W^{\prime} Z_{0}^{1}=0 .
$$

On impose les conditions initiales:

$$
\begin{aligned}
& \sum_{t^{\prime}} Z_{0}^{t^{\prime}}\left|=0=\cdots=\sum_{t^{\prime}} Z_{4}^{t^{\prime}}\right|=0 ; \quad \sum_{t} Y_{0}^{t}\left|+\sum_{t^{\prime}} Z_{5}^{t^{\prime}}\right|=0 ; \quad \ldots ; \\
& \sum_{t} Y_{3}^{t}\left|+\sum_{t^{\prime}} Z_{8}^{t^{\prime}}\right|=I ; \quad \ldots ;
\end{aligned}
$$

on en déduit:

$$
\begin{aligned}
& \sum_{t^{\prime}} Z_{0}^{t^{\prime} 1}\left|=0 ; \quad \ldots ; \quad \sum_{t^{\prime}} D_{0} \chi^{t^{\prime}} Z_{0}^{t^{\prime} 1}\right|=0 ; \quad \sum_{t} Y_{0}^{t 4} \mid=0 ; \quad \ldots ; \\
& \left.\sum_{t^{\prime}}\left(D_{0} \chi^{t^{\prime}}\right)^{2} Z_{0}^{t^{\prime} 1}|=| \eta\right|^{2} I^{3}, \quad-|\eta| \sum_{t} D_{0} \psi^{t} Y_{0}^{t 4}\left|-\frac{b_{01}^{4}}{b_{04}^{5}} \sum_{t^{\prime}}\left(D_{0} \chi^{t^{\prime}}\right)^{2} Z_{0}^{1}\right|=|\eta|^{2} I^{5}
\end{aligned}
$$

d'où l'on déduit les $Z_{0}^{t^{\prime} 1} \mid$ et les $Y_{0}^{t 4} \mid$ et tous les coefficients.

On distingue les cas

2b) $\quad N$ est formé des points $0,(2,0),(3,0),(4,0)$.

2b) ${ }_{1} b_{04}^{3}$ ou $b_{01}^{5} \neq 0$.

b) ${ }_{1}, b_{04}^{3} \neq 0, C^{\prime}=0$.

b) $1^{\prime \prime} b_{01}^{5} \neq 0, C^{\prime \prime}=0$.

$$
\begin{aligned}
f & =\sum_{1 \leq t \leq 2} f_{1}^{t}+f_{2} ; \\
f_{1}^{t} & =e^{\psi^{t}}\left[Y_{0}^{t}+\cdots+Y_{k}^{t}+\cdots\right]
\end{aligned}
$$

$\left(D_{0} \psi^{t}\right)^{2}=\left(b_{02}^{3}+b_{04}^{5}\right)|\eta| ; \psi^{t}$ est d'ordre $1 / 2 ; Y_{k}^{t}$ est d'ordre: $\frac{2-k}{2} ;$

$$
f_{2}=Z_{0}+\cdots+Z_{k}+\cdots
$$

$Z_{k}$ est d'ordre: $\frac{4-k}{2}$. 
b) ${ }_{1^{\prime}} b_{04}^{3} \neq 0 . \quad$ On a les systèmes en $Y$ et $Z$ du cas a) $)_{1^{\prime}}$. On obtient:

$$
Y_{0}^{2}=Y_{0}^{3}=Y_{0}^{5}=0 ; \quad \ldots ; \quad Y_{0}^{4}=0 ;
$$

à l'étape (3):

$$
\left[\left(D_{0} \psi\right)^{4}-\left(D_{0} \psi\right)^{2}\left(b_{02}^{3}+b_{04}^{5}\right)|\eta|\right] Y_{0}^{1}=0,
$$

laisse $Y_{0}^{1}$ libre; à l'étape (4):

$$
2\left(D_{0} \psi\right)^{4} D_{0} Y_{0}^{1}+W Y_{0}^{1}=0 .
$$

Pour les $Z$ on obtient:

$$
Z_{0}^{2}=Z_{0}^{3}=Z_{0}^{5}=0 ; \quad \ldots ; \quad Z_{0}^{4}=0 ;
$$

à l'étape (4), $Z_{0}^{1}$ reste libre; ...; à l'étape (6), on a:

$$
\left(b_{02}^{3}+b_{04}^{5}\right) D_{0}^{3} Z_{0}^{1}+W^{\prime} D_{0}^{2} Z_{0}^{1}+W^{\prime \prime} D_{0} Z_{0}^{1}+W^{\prime \prime \prime} Z_{0}^{1}=0 .
$$

On impose:

$$
\begin{array}{lll}
Z_{0} \mid=0 ; & Z_{1} \mid=0 ; & \sum_{t} Y_{0}^{t}\left|+Z_{2}\right|=0 \\
\sum_{t} Y_{1}^{t}+Z_{3} \mid=0 ; & \sum_{t} Y_{2}^{t} \mid+Z_{4}=I ; & \ldots ;
\end{array}
$$

on en déduit:

$$
\begin{aligned}
& Z_{0}^{1}\left|=0 ; \quad D_{0} Z_{0}^{1}\right|=0 ; \quad \sum_{t} D_{0} \psi^{t} Y_{0}^{t 1} \mid=0 \\
& \left(b_{02}^{3}+b_{04}^{5}\right)|\eta| \sum_{t} Y_{0}^{t 1}\left|+D_{0}^{2} Z_{0}^{1}\right|=|\eta|^{2} I^{3} \\
& \frac{\left(b_{04}^{3}+b_{04}^{5}\right)}{b_{04}^{3}} b_{04}^{5}|\eta| \sum_{t} Y_{0}^{t 1}\left|-\frac{b_{02}^{3}}{b_{04}^{3}} D_{0}^{2} Z_{0}^{1}\right|=|\eta|^{2} I^{5}
\end{aligned}
$$

d'où l'on déduit $Y_{0}^{t 1} \mid$ et $D_{0} Z_{0}^{1} \mid$ et tous les coefficients.

b) ${ }_{1^{\prime \prime}} b_{01}^{5} \neq 0$. Ce cas est analogue au précédent. 
2b) $)_{2^{\prime}} b_{04}^{3}=b_{01}^{5}=0 ; b_{02}^{3}=0, b_{04}^{5} \neq 0 ; b_{04}^{5} b_{01}^{4}-b_{11}^{3}|\eta|=0$.

$$
\begin{aligned}
f & =\sum_{1 \leq t \leq 2} f_{1}^{t}+f_{2} \\
f_{1}^{t} & =e^{\psi^{t}}\left[Y_{0}^{t}+\cdots+Y_{k}^{t}+\cdots\right]
\end{aligned}
$$

$\left(D_{0} \psi^{t}\right)^{2}=b_{04}^{5}|\eta| ; \psi^{t}$ est d'ordre $1 / 2 ; Y_{k}^{t}$ est d'ordre $\frac{1-k}{2} ;$

$$
f_{2}=Z_{0}+\cdots+Z_{k}+\cdots
$$

$Z_{k}$ est d'ordre $\frac{4-k}{2}$.

Le système en $Y$ du cas 1a) donne:

$$
Y_{0}^{2}=Y_{0}^{3}=Y_{0}^{5} ; \quad \ldots ;
$$

à la $3^{\text {ème }}$ étape, on a:

$$
b_{04}^{5} Y_{0}^{1}-b_{05}^{3} Y_{0}^{4}=0 \quad \text { et } \quad 2 D_{0} \psi D_{0} Y_{0}^{4}+W Y_{0}^{4}=0 .
$$

Le système en $Z$ donne:

$$
Z_{0}^{2}=Z_{0}^{3}=Z_{0}^{5}=0 ; \quad \ldots ; \quad Z_{0}^{4}=0
$$

à la $6^{\text {ème }}$ étape:

$$
D_{0}^{3} Z_{0}^{1}+W D_{0}^{2} Z_{0}^{1}+W^{\prime} D_{0} Z_{0}^{1}+W^{\prime \prime} Z_{0}^{1}=0
$$

On impose:

$$
Z_{0}\left|=0 ; \quad Z_{1}\right|=0 ; \quad Z_{2}\left|=0 ; \quad \sum_{t} Y_{0}^{t}\right|+Z_{3}\left|=0 ; \quad \sum_{t} Y_{1}^{t}\right|+Z_{4} \mid=I ; \quad \ldots
$$

on en déduit:

$$
\begin{aligned}
& Z_{0}^{1}\left|=0 ; \quad D_{0} Z_{0}^{1}\right|=0 ; \quad \ldots ; \quad \sum_{t} Y_{0}^{t 4}\left|=0 ; \quad D_{0}^{2} Z_{0}^{1}\right|=|\eta|^{2} I^{3} \\
& -|\eta| \sum_{t} D_{0} \psi^{t} Y_{0}^{t 4}\left|-\frac{b_{01}^{4}}{b_{04}^{5}} D_{0}^{2} Z_{0}^{1}\right|=|\eta|^{2} I^{5}
\end{aligned}
$$

on obtient les $Z$ et les $Y$. 
2b) $)_{2^{\prime \prime}} b_{04}^{3}=b_{01}^{5}=0 ; b_{02}^{3} \neq 0, b_{04}^{5}=0$.

$$
\begin{aligned}
f & =\sum_{1 \leq t \leq 2} f_{1}^{t}+f_{2} \\
f_{1}^{t} & =e^{\psi^{t}}\left[Y_{0}^{t}+\cdots+Y_{k}^{t}+\cdots\right]
\end{aligned}
$$

$\left(D_{0} \psi^{t}\right)^{2}=b_{02}^{3}|\eta| ; \psi^{t}$ est d'ordre $1 / 2 ; Y_{k}^{t}$ est d'ordre $\frac{2-k}{2} ;$

$$
f_{2}=Z_{0}+\cdots+Z_{k}+\cdots
$$

$Z_{k}$ est d'ordre $\frac{2-k}{2}$.

On a:

$$
Y_{0}^{2}=Y_{0}^{3}=Y_{0}^{5}=0 ; \quad \ldots ; \quad Y_{0}^{4}=0
$$

à la $3^{\text {ème }}$ étape

$$
D_{0} \psi Y_{1}^{4}+b_{01}^{4} Y_{0}^{1}=0
$$

à la $4^{\text {ème }}$ étape:

$$
2\left(D_{0} \psi\right)^{2} D_{0} Y_{0}^{1}+W Y_{0}^{1}=0
$$

On a aussi:

$$
Z_{0}^{2}=Z_{0}^{3}=Z_{0}^{5}=0
$$

à la $4^{\text {ème }}$ étape:

$$
\begin{aligned}
& b_{02}^{3} D_{0} Z_{0}^{1}+b_{05}^{3} D_{0} Z_{0}^{4}+\left(b_{05}^{3} b_{01}^{4}-b_{11}^{3}|\eta|\right) Z_{0}^{1}-b_{14}^{3}|\eta| Z_{0}^{4}=0 \\
& D_{0}^{2} Z_{0}^{4}+b_{01}^{4} D_{0} Z_{0}^{1}+\left(D_{0} b_{01}^{4}-b_{11}^{5}|\eta|\right) Z_{0}^{1}-b_{14}^{5}|\eta| Z_{0}^{4}=0
\end{aligned}
$$

on doit déterminer les $Y_{0}^{t 1} \mid$ et les $Z_{0}^{1}\left|, Z_{0}^{4}\right|, D_{0} Z_{0}^{4} \mid$; on impose:

$$
\sum_{t} Y_{0}^{t}\left|+Z_{0}\right|=0 ; \quad \sum_{t} Y_{1}^{t}\left|+Z_{1}\right|=0 ; \quad \sum_{t} Y_{2}^{t}+Z_{2} \mid=I ; \quad \ldots ;
$$

d'où:

$$
\begin{aligned}
& \sum_{t} Y_{0}^{t 1}\left|+Z_{0}^{1}\right|=0 ; \quad Z_{0}^{4}\left|=0 ; \quad \sum_{t} D_{0} \psi^{t} Y_{0}^{t 1}\right|=0 \\
& b_{02}^{3} \sum_{t} Y_{0}^{t 1}|=| \eta\left|I^{3}, \quad b_{01}^{4} \sum_{t} Y_{0}^{t 1}\right|+D_{0} Z_{0}^{4}|=| \eta \mid I^{5}
\end{aligned}
$$

d'où les $Y_{0}^{t 1} \mid$ et $D_{0} Z_{0}^{4}\left|, Z_{0}^{1}\right|$; on obtient tous les coefficients. 
3.4. Cas $(\mathbf{L G})_{5 / 2}$. On pose:

si $b_{04}^{3} \neq 0, C^{\prime}=b_{05}^{3} b_{04}^{5} b_{01}^{4}-b_{04}^{3} D_{0} b_{01}^{4}+\left(b_{04}^{3} b_{11}^{5}+b_{02}^{3} b_{11}^{3}\right)|\eta|$

si $b_{01}^{5} \neq 0, C^{\prime \prime}=b_{05}^{3} b_{04}^{5} b_{01}^{4}+b_{02}^{3} b_{01}^{5} D_{0}\left(\frac{b_{04}^{5}}{b_{01}^{5}}\right)+\left(b_{14}^{3} b_{01}^{5}+b_{02}^{3} b_{11}^{3}\right)|\eta|$;

$C=C^{\prime}$ ou $C^{\prime \prime}$ selon le cas

$$
f=\sum e^{\psi_{1}^{t}+\psi_{2}^{t}}\left[Y_{0}^{t}+\cdots+Y^{t}+\cdots\right]
$$

$\left(D_{0} \psi_{1}^{t}\right)^{5}=-C|\eta|^{2} ; \psi_{1}^{t}$ est d'ordre $2 / 5 ; D_{0} \psi_{2}^{t}$ sera précisé ci-dessous; $\psi_{2}^{t}$ est d'ordre $1 / 5 ; Y_{k}^{t}$ est d'ordre: $\frac{7-k}{5}$.

Le système en $Y$ est celui du §1.6.

1) $b_{04}^{3} \neq 0$. On obtient:

$$
Y_{0}^{2}=Y_{0}^{3}=Y_{0}^{5}=0 ; \quad \ldots ; \quad Y_{0}^{4}=0 ;
$$

à la $10^{\text {ème }}$ étape, on a:

$$
\left[\left(D_{0} \psi_{1}\right)^{5}-C^{\prime}|\eta|^{2}\right] Y_{0}^{1}=0
$$

qui laisse $Y_{0}^{1}$ libre; à la $11^{\text {ème }}$ étape, on obtient:

$$
\left[5 D_{0} \psi_{2}\left(D_{0} \psi_{1}\right)^{2}+|\eta|\left(b_{11}^{3}|\eta|-2 b_{04}^{3} D_{0}\left(\frac{b_{02}^{3}}{b_{04}^{3}}\right)\right)\right] Y_{0}^{1}=0 ;
$$

$Y_{0}^{1}$ reste libre par le choix de $D_{0} \psi_{2} ;$ à la $12^{\text {ème }}$ étape, on a:

$$
5\left(D_{0} \psi_{1}\right)^{4} D_{0} Y_{0}^{1}+W Y_{0}^{1}=0 .
$$

On impose:

$$
\sum_{t} Y_{0}^{t}\left|=0 ; \quad \ldots ; \quad \sum_{t} Y_{6}^{t}\right|=0 ; \quad \sum_{t} Y_{7}^{t} \mid=I ; \quad \ldots ;
$$

on obtient:

$$
\begin{gathered}
\sum_{t} Y_{0}^{t 1}\left|=\sum_{t}\left(D_{0} \psi^{t}\right) Y_{0}^{t 1}\right|=\sum_{t}\left(D_{0} \psi^{t}\right)^{2} Y_{0}^{t 1}\left|=\sum_{t}\left(D_{0} \psi^{t}\right)^{3} Y_{0}^{t 1}\right|=0 \\
\left.\sum_{t}\left(D_{0} \psi^{t}\right)^{4} Y_{0}^{t 1}|=| \eta\right|^{2} b_{04}^{3} I^{5}
\end{gathered}
$$

d'où les $Y_{0}^{t 1} \mid$ et tous les coefficients $Y$.

2) $b_{01}^{5} \neq 0$. On remarque que:

$$
b_{01}^{5} Y_{0}^{1}+b_{04}^{5} Y_{0}^{4}=0
$$

et on obtient comme précédemment:

$$
D_{0} Y_{0}^{4}+W Y_{0}^{4}=0
$$

on détermine les conditions initiales comme dans le cas 1 ). 


\subsection{Cas $(\mathbf{L G})_{3}$.}

1) $b_{04}^{3}$ ou $b_{01}^{5} \neq 0$; on pose:

Si $b_{04}^{3} \neq 0, C_{3}^{\prime}=b_{11}^{3}|\eta|-2 b_{04}^{3} D_{0}\left(\frac{b_{02}^{3}}{b_{04}^{3}}\right)$;

Si $b_{01}^{5} \neq 0, C_{3}^{\prime \prime}=b_{11}^{3}|\eta|+D_{0} b_{02}^{3}+2 b_{01}^{5} D_{0}\left(\frac{b_{02}^{3}}{b_{01}^{5}}\right)$;

$C_{3}=C_{3}^{\prime}$ ou $C_{3}^{\prime \prime}$

$$
\begin{aligned}
f & =\sum_{1 \leq t \leq 3} f_{1}^{t}+f_{2} \\
f_{1}^{t} & =e^{\psi^{t}}\left[Y_{0}^{t}+\cdots Y_{k}^{t}+\cdots\right]
\end{aligned}
$$

$\left(D_{0} \psi^{t}\right)^{3}=-C_{3}|\eta| ; \psi^{t}$ est d'ordre $1 / 3 ; Y_{k}^{t}$ est d'ordre $\frac{5-k}{3}$;

$$
f_{2}=Z_{0}+\cdots+Z_{k}+\cdots ;
$$

$Z_{k}$ est d'ordre $\frac{6-k}{3}$.

1) $1 \quad b_{04}^{3} \neq 0$. Les systèmes en $Y$ et $Z$ sont ceux du $\S 1.7$

$$
Y_{0}^{2}=Y_{0}^{3}=Y_{0}^{5}=0 ; \quad \ldots ; \quad Y_{0}^{4}=0 ;
$$

à la $7^{\text {ème }}$ étape, on a:

$$
\left[\left(D_{0} \psi\right)^{3}+C_{3}^{\prime}|\eta|\right] Y_{0}^{1}=0
$$

$Y_{0}^{1}$ reste libre; à la $8^{\text {ème }}$ étape, on a:

$$
\begin{gathered}
3\left(D_{0} \psi\right)^{4} D_{0} Y_{0}^{1}+W Y_{0}^{1}=0 \\
Z_{0}^{2}=Z_{0}^{3}=Z_{0}^{5}=0 ; \quad \ldots ; \quad Z_{0}^{4}=0
\end{gathered}
$$

à la $9^{\text {ème }}$ étape on obtient:

$$
C_{3}^{\prime} D_{0}^{2} Z_{0}^{1}+W^{\prime} D_{0} Z_{0}^{1}+W^{\prime \prime} Z_{0}^{1}=0 .
$$

On impose:

$$
Z_{0}\left|=0 ; \quad \sum_{t} Y_{0}^{t}\right|+Z_{1}\left|=0 ; \quad \ldots ; \quad \sum_{t} Y_{5}^{t}\right|+Z_{6} \mid=I ; \quad \ldots ;
$$

on obtient:

$$
\begin{aligned}
& Z_{0}^{1}\left|=0 ; \quad \sum_{t} D_{0} \psi^{t} Y_{0}^{t 1}\right|+D_{0} Z_{0}^{1}\left|=0 ; \quad \sum_{t} Y_{0}^{t 1}\right|=0 \\
& \sum_{t}\left(D_{0} \psi^{t}\right)^{2} Y_{0}^{t 1}\left|=0 ; \quad \sum_{t} Y_{5}^{t 5}\right|+Z_{6}^{5} \mid=I^{5}
\end{aligned}
$$

donne:

$$
\left.C_{3}^{\prime} \sum_{t} D_{0} \psi^{t} Y_{0}^{t 1}\left|=-b_{04}^{3}\right| \eta\right|^{2} I^{5}
$$

d'où les $Y_{0}^{t 1} \mid$ et $D_{0} Z_{0}^{1} \mid$ et tous les coefficients. 
1) $b_{01}^{5} \neq 0$. Ce cas se traite de façon analogue au précédent

2) $b_{04}^{3}=b_{01}^{5}=0 ; \quad$ on rappelle que: $b_{02}^{3}=b_{04}^{5}=0 ; b_{11}^{3} \neq 0$;

$$
\begin{aligned}
f & =\sum_{1 \leq t \leq 3} f_{1}^{t}+f_{2} ; \\
f_{1}^{t} & =e^{\psi^{t}}\left[Y_{0}^{t}+\cdots+Y_{k}^{t}+\cdots\right],
\end{aligned}
$$

$\left(D_{0} \psi^{t}\right)^{3}=-b_{11}^{3}|\eta|^{2} ; \psi^{t}$ est d'ordre $1 / 3 ; Y_{k}^{t}$ est d'ordre: $\frac{4-k}{3}$;

$$
f_{2}=Z_{0}+\cdots+Z_{k}+\cdots
$$

$Z_{k}$ est d'ordre $\frac{3-k}{3}$. Le système différentiel des $Y$ donne:

$$
Y_{0}^{2}=Y_{0}^{3}=Y_{0}^{5}=0 ; \quad \cdots ; \quad Y_{0}^{4}=0 ;
$$

à la $6^{\text {ème }}$ étape, $3^{\text {ème }}$ ligne:

$$
\left[\left(D_{0} \psi^{t}\right)^{3}+b_{11}^{3}|\eta|^{2}\right] Y_{0}^{1}=0
$$

laisse $Y_{0}^{1}$ libre; à la $7^{\text {ème }}$ étape on a:

$$
3\left(D_{0} \psi^{t}\right)^{2} D_{0} Y_{0}^{1}+W Y_{0}^{1}=0 .
$$

Le système différentiel des $Z$ donne:

$$
Z_{0}^{2}=Z_{0}^{3}=Z_{0}^{5}=0
$$

Ensuite, on distingue:

2) ${ }_{1} b_{05}^{3} \neq 0$, on obtient:

$$
\begin{aligned}
& b_{05}^{3} D_{0} Z_{0}^{4}+b_{05}^{3} b_{01}^{4} Z_{0}^{1}-b_{11}^{3}|\eta| Z_{0}^{1}-b_{14}^{3}|\eta| Z_{0}^{4}=0 \\
& D_{0}^{2} Z_{0}^{4}+b_{01}^{4} D_{0} Z_{0}^{1}+\left(D_{0} b_{01}^{4}-b_{11}^{5}|\eta|\right) Z_{0}^{1}-b_{14}^{5}|\eta| Z_{0}^{4}=0
\end{aligned}
$$

Ce système se transforme en un système de la forme:

$$
\begin{aligned}
& \frac{b_{11}^{3}}{b_{05}^{3}} D_{0} Z_{0}^{1}+W Z_{0}^{1}+W^{\prime} Z_{0}^{4}=0 \\
& D_{0} Z_{0}^{4}+\left[b_{01}^{4}-\frac{b_{11}^{3}|\eta|}{b_{05}^{3}}\right] Z_{0}^{1}-\frac{b_{14}^{3}}{b_{05}^{3}} Z_{0}^{4}=0
\end{aligned}
$$

Il suffit donc de connaître $Z_{0}^{1} \mid$ et $Z_{0}^{4} \mid$. 
On impose les conditions:

$$
\sum_{t} Y_{0}^{t}\left|=0 ; \quad \sum_{t} Y_{1}^{t}\right|+Z_{0}=0 ; \quad \ldots ; \quad \sum_{t} Y_{4}^{t}\left|+Z_{3}\right|=I ; \quad \ldots ;
$$

on obtient:

$$
\begin{array}{ll}
\sum_{t} Y_{0}^{t 1} \mid=0 ; & \left.\frac{b_{01}^{4}}{b_{11}^{3}} \sum_{t}\left(D_{0} \psi^{t}\right)^{2} Y_{0}^{t 1}|+| \eta\right|^{2} Z_{0}^{4} \mid=0 \\
\sum_{t} D_{0} \psi^{t} Y_{0}^{t 1} \mid=0 ; & \left.\sum_{t}\left(D_{0} \psi^{t}\right)^{2} Y_{0}^{t 1}|=| \eta\right|^{2} I^{3}
\end{array}
$$

on en déduit les $Y_{0}^{t 1} \mid$ et $Z_{0}^{4} \mid$

$$
b_{11}^{5} \frac{\sum_{t}\left(D_{0} \psi^{t}\right)^{2} Y_{0}^{t 1}}{b_{11}^{3}}\left|-D_{0} Z_{01}^{4}\right|-b_{01}^{4} Z_{0}^{1} \mid=I^{5}
$$

on en déduit:

$$
\frac{b_{11}^{5}}{b_{11}^{3}}\left|I^{3}-\frac{b_{11}^{3}}{b_{05}^{3}} Z_{0}^{1}\right|+\frac{b_{14}^{3} b_{01}^{4}}{b_{05}^{3} b_{11}^{3}} \mid I^{3}=I^{5}
$$

on obtient $Z_{0}^{1} \mid$ et par suite tous les coefficients.

2) ${ }_{2} \quad b_{05}^{3}=0$, on obtient:

$$
D_{0}^{2} Z_{0}^{4}+W D_{0} Z_{0}^{4}+W^{\prime} Z_{0}^{4}=0
$$

Les conditions initiales imposées au cas 2) ${ }_{1}$ permettent d'obtenir les $Y_{0}^{t 1}\left|, Z_{0}^{4}\right|$ et $D_{0} Z_{0}^{4} \mid$.

\subsection{Cas $(\mathbf{L G})_{4}$.}

1) $b_{04}^{3}$ ou $b_{01}^{5} \neq 0$.

$$
\begin{aligned}
f & =\sum_{1 \leq t \leq 4} f_{1}^{t}+f_{2} ; \\
f_{1}^{t} & =e^{\psi^{t}}\left[Y_{0}^{t}+\cdots+Y_{k}^{t}+\cdots\right] ;
\end{aligned}
$$

$\left(D_{0} \psi^{t}\right)^{4}=-C_{4}^{\prime}|\eta| ; \psi^{t}$ est d'ordre $1 / 4, Y_{k}^{t}$ est d'ordre $\frac{8-k}{4}$

$$
f_{2}=Z_{0}+\cdots+Z_{k}+\cdots
$$

$Z_{k}$ est d'ordre: $\frac{8-k}{4}$. 
a) $\quad b_{04}^{3} \neq 0$. Les systèmes en $Y$ et $Z$ sont ceux du $\S 1.8$.

Les calculs deviennent longs. On obtient en bref:
(0) $Y_{0}^{2}=Y_{0}^{3}=Y_{0}^{5}=0$;
(4) $Y_{0}^{4}=0$
(7) (8) (9) (10)

laissent $Y_{0}^{1}$ libres du fait des conditions satisfaites par les coefficients $b_{0}, b_{1}$ dans ce cas; à la $11^{\text {ème }}$ étape on obtient:

$$
\left[\left(D_{0} \psi\right)^{4}+C_{4}^{\prime}|\eta|\right] Y_{0}^{1}=0
$$

qui laisse $Y_{0}^{1}$ libre; à la $12^{\text {ème }}$ étape, on a:

$$
4\left(D_{0} \psi\right)^{4} D_{0} Y_{0}^{1}+W Y_{0}^{1}=0 .
$$

On a aussi:
(0) $\quad Z_{0}^{2}=Z_{0}^{3}=Z_{0}^{5}=0$;
$\ldots$
(4) $Z_{0}^{4}=0$;
(7) (8) (9) (10)

laissent $Z_{0}^{1}$ libre; à la $12^{\text {ème }}$ étape, on obtient:

$$
C_{4}^{\prime} D_{0} Z_{0}^{1}+W^{\prime} Z_{0}^{1}=0 .
$$

On impose aux données initiales les conditions:

$$
\sum_{t} Y_{0}^{t}\left|+Z_{0}\right|=0 ; \quad \sum_{t} Y_{1}^{t}\left|+Z_{1}\right|=0 ; \quad \ldots ; \quad \sum_{t} Y_{8}^{t}\left|+Z_{8}\right|=I ; \quad \ldots
$$

on obtient successivement:

$$
\begin{array}{ll}
\sum_{t} Y_{0}^{t 1}\left|+Z_{0}^{1}\right|=0 ; & \sum_{t} D_{0} \psi^{t} Y_{0}^{t 1} \mid=0 \\
\sum_{t}\left(D_{0} \psi^{t}\right)^{3} Y_{0}^{t 1} \mid=0 ; & \sum_{t}\left(D_{0} \psi^{t}\right)^{2} Y_{0}^{t 1} \mid=0 \\
-\left.C_{4}^{\prime} \sum_{t} Y_{0}^{t 1}\left|+\frac{b_{02}^{3}}{b_{04}^{3}}\right| \eta\right|^{2} I^{3}=|\eta|^{2} I^{5} ; &
\end{array}
$$

on en déduit les $Y_{0}^{t 1} \mid$ et $Z_{0}^{1} \mid$, d'où tous les coefficients.

b) $\quad b_{01}^{5} \neq 0$. Les calculs sont analogues, mais encore plus longs; on remplace $C_{4}^{\prime}$ par $C_{4}^{\prime \prime}$. 
2) $b_{04}^{3}=b_{01}^{5}=b_{02}^{3}=b_{04}^{5}=b_{11}^{3}=0 ; b_{14}^{3} b_{01}^{4}+b_{05}^{3} b_{11}^{5} \neq 0$.

$$
\begin{aligned}
f & =\sum_{1 \leq t \leq 4} f_{1}^{t}+f_{2} \\
f_{1}^{t} & =e^{\psi^{t}}\left[Y_{0}^{t}+\cdots+Y_{k}^{t}+\cdots\right]
\end{aligned}
$$

$\left(D_{0} \psi^{t}\right)^{4}=\left(b_{14}^{3} b_{01}^{4}+b_{05}^{3} b_{11}^{5}\right)|\eta|^{2} ; \psi^{t}$ est d'ordre $1 / 4 ; Y_{k}^{t}$ est d'ordre $\frac{6-k}{4} ;$

$$
f_{2}=Z_{0}+\cdots+Z_{k}+\cdots
$$

$Z_{k}$ est d'ordre $\frac{4-k}{4}$.

Pour les $Y$, on a:

$$
Y_{0}^{2}=Y_{0}^{3}=Y_{0}^{5}=0 ; \quad \ldots ; \quad Y_{0}^{4}=0 ;
$$

à la $9^{\text {ème }}$ étape, $3^{\text {ème }}$ ligne:

$$
\left[\left(D_{0} \psi\right)^{4}-\left(b_{14}^{3} b_{01}^{4}+b_{05}^{3} b_{11}^{5}\right)|\eta|^{2}\right] Y_{0}^{1}=0
$$

laisse $Y_{0}^{1}$ libre; à la $10^{\text {ème }}$ étape, on a:

$$
4\left(D_{0} \psi\right)^{2} D_{0} Y_{0}^{1}+W Y_{0}^{1}=0 .
$$

Pour les $Z$, on a:

$$
Z_{0}^{2}=Z_{0}^{3}=Z_{0}^{5}=0
$$

à la $8^{\text {ème }}$ étape, si $b_{05}^{3} \neq 0, Z_{0}^{1}$ s'exprime à l'ordre de $Z_{0}^{4}$ et il reste:

$$
D_{0} Z_{0}^{4}+W^{\prime} Z_{0}^{4}=0
$$

si $b_{05}^{3}=0$, on a:

$$
Z_{0}^{4}=0 \quad \text { et } \quad D_{0} Z_{0}^{1}+W^{\prime \prime} Z_{0}^{1}=0
$$

On impose aux données les conditions:

$$
\begin{array}{llll}
\sum_{t} Y_{0}^{t} \mid=0 ; & \sum_{t} Y_{1}^{t} \mid=0 ; & \sum_{t} Y_{2}^{t}\left|+Z_{0}\right| & =0 ; \\
\sum_{t} Y_{3}^{t}\left|+Z_{1}\right|=0 ; & \ldots ; & \sum_{t} Y_{6}^{t}\left|+Z_{4}\right|=I ; & \ldots
\end{array}
$$

on distingue les cas: 
$b_{05}^{3} \neq 0$; on obtient:

$$
\begin{array}{ll}
\sum_{t} Y_{0}^{t 1} \mid=0 ; & \sum_{t} D_{0} \psi^{t} Y_{0}^{t 1} \mid=0 \\
\sum_{t}\left(D_{0} \psi^{t}\right)^{3} Y_{0}^{t 1} \mid=0 ; & \left.\sum_{t}\left(D_{0} \psi^{t}\right)^{2} Y_{0}^{t 1}|=| \eta\right|^{2} I^{3}
\end{array}
$$

et à l'aide de $\sum_{t} Y_{6}^{t 5}\left|+Z_{4}^{5}\right|=I^{5}$, on obtient $Z_{0}^{4} \mid$, d'où tous les coefficients.

$b_{05}^{3}=0$; on obtient:

$$
\begin{aligned}
& \sum_{t} Y_{0}^{t 1}\left|=0 ; \quad \sum_{t}\left(D_{0} \psi^{t}\right)^{3} Y_{0}^{t 1}\right|=0 ; \\
& \sum_{t} D_{0} \psi^{t} Y_{0}^{t 1}\left|=0 ; \quad \sum_{t}\left(D_{0} \psi^{t}\right)^{2} Y_{0}^{t 1}\right|=|\eta|^{2} I^{3}
\end{aligned}
$$

et $\sum_{t} Y_{6}^{t 5}\left|+Z_{4}^{5}\right|=I^{5}$ donne $Z_{0}^{1} \mid$.

\subsection{Cas $(\mathbf{L G})_{5}$.}

1) $b_{04}^{3}$ ou $b_{01}^{5} \neq 0$.

$$
f=\sum_{1 \leq t \leq 5} e^{\psi_{t}}\left[Y_{0}+\cdots+Y_{k}+\cdots\right]
$$

$\left(D_{0} \psi^{t}\right)^{5}=-C_{5}|\eta| ; \psi^{t}$ est d'ordre $1 / 5 ; Y_{k}^{t}$ est d'ordre $\frac{11-k}{5}$. Le système en $Y$ est celui du $\S 1.9$. Les calculs deviennent longs et assez compliqués; nous les résumerons.

a) $b_{04}^{3} \neq 0$.

$$
\text { (0) } Y_{0}^{2}=Y_{0}^{3}=Y_{0}^{5}=0 ; \quad(5) \quad Y_{0}^{4}=0 ; \quad \ldots ;
$$

à la $15^{\text {ème }}$ étape on obtient:

$$
\left[\left(D_{0} \psi\right)^{5}+C_{5}^{\prime}|\eta|\right] Y_{0}^{1}=0
$$

$Y_{0}^{1}$ reste libre; à la $16^{\text {ème }}$ étape, on a:

$$
5\left(D_{0} \psi\right)^{4} D_{0} Y_{0}^{1}+W Y_{0}^{1}=0 .
$$

On impose aux données les conditions initiales:

$$
\sum_{t} Y_{0}^{t}\left|=\sum_{t} Y_{1}^{t}\right|=\cdots=\sum_{t} Y_{10}^{t}\left|=0 ; \quad \sum_{t} Y_{11}^{t}\right|=I ; \quad \ldots ;
$$

on en déduit:

$$
\begin{aligned}
& \sum_{t} Y_{0}^{t 1}\left|=0 ; \quad \sum_{t} D_{0} \psi^{t} Y_{0}^{t 1}\right|=0 ; \quad \sum_{t}\left(D_{0} \psi^{t}\right)^{2} Y_{0}^{t 1} \mid=0 ; \\
& \sum_{t}\left(D_{0} \psi^{t}\right)^{3} Y_{0}^{t 1}\left|=0 ; \quad \sum_{t}\left(D_{0} \psi^{t}\right)^{4} Y_{0}^{t 1}\right|=|\eta|^{3} I
\end{aligned}
$$

d'où les $Y_{0}^{t 1}$ et tous les $Y$. 
b) $b_{01}^{5} \neq 0$.
(0) $Y_{0}^{2}=Y_{0}^{3}=Y_{0}^{5}=0$;
(5) $\quad b_{01}^{5} Y_{0}^{1}+b_{04}^{5} Y_{0}^{4}=0, \quad \ldots ;$

à la $15^{\text {ème }}$ étape, $Y_{0}^{4}$ reste libre et à la $16^{\text {ème }}$ étape; $C_{5}^{\prime}$ est remplacé par $C_{5}^{\prime \prime}$; on a l'équation de propagation. Les conditions initiales sont obtenues comme précédemment et ainsi tous les coefficients.

2) $b_{04}^{3}=b_{01}^{5}=0 . \quad f$ a la même forme que dans le cas 1a);

$$
\left(D_{0} \psi^{t}\right)^{5}=-\left(b_{05}^{3} b_{14}^{5} b_{01}^{4}+b_{14}^{3} b_{11}^{5}|\eta|+b_{14}^{3} D_{0} b_{01}^{4}+b_{05}^{3} D_{0} b_{11}^{5}\right)|\eta|^{2}
$$

$\psi^{t}$ est d'ordre $1 / 5 ; Y_{k}^{t}$ est d'ordre $\frac{8-k}{5}$.

On a:

$$
\text { (0) } Y_{0}^{2}=Y_{0}^{3}=Y_{0}^{5}=0 ; \quad \ldots ; \quad \text { (8) } Y_{0}^{4}=0, \quad \ldots \text {; }
$$

à la $12^{\text {ème }}$ étape, le choix de $D_{0} \psi$ laisse $Y_{0}^{1}$ libre; à la $13^{\text {ème }}$ étape, on a:

$$
5\left(D_{0} \psi\right)^{4} D_{0} Y_{0}^{1}+W Y_{0}^{1}=0 .
$$

On impose aux données les conditions initiales:

$$
\sum_{t} Y_{0}^{t}\left|=\cdots=\sum_{t} Y_{7}^{t}\right|=0 ; \quad \sum_{t} Y_{8}^{t} \mid=I ; \quad \cdots
$$

on en déduit:

$$
\begin{array}{lll}
\sum_{t} Y_{0}^{t 1} \mid=0 ; & \sum_{t}\left(D_{0} \psi^{t}\right)^{4} Y_{0}^{t} \mid=0 ; & \sum_{t} D_{0} \psi^{t} Y_{0}^{t 1} \mid=0 \\
\sum_{t}\left(D_{0} \psi^{t}\right)^{3} Y_{0}^{t 1} \mid=0 ; & \left.\sum_{t}\left(D_{0} \psi^{t}\right)^{2} Y_{0}^{t 1}|=| \eta\right|^{2} I^{3}
\end{array}
$$

on en déduit les 5 valeurs initiales $Y_{0}^{t 1} \mid$, les $Y_{0}^{t 1} \mid$ et tous les coefficients.

3.8. Cas L. Toutes les conditions $\mathrm{L}$ sont satisfaites. Le diagramme est vide.

$$
f=Y_{0}+\cdots+Y_{k}+\cdots
$$

Le système en $Y$ est le même que dans les cas L des paragraphes précédents; il a été détaillé au §1.10, pour les étapes (0) (1) (2) (3) (4).

1) $b_{04}^{3}$ ou $b_{01}^{5} \neq 0 . \quad Y_{k}$ est d'ordre $3-k$ 
a) $b_{04}^{3} \neq 0 ; b_{01}^{5}=0$.

(0) $Y_{0}^{2}=Y_{0}^{3}=Y_{0}^{5}=0$;

(1) $|\eta| Y_{1}^{2}+D_{0} Y_{0}^{1}=0, \quad Y_{0}^{4}=0, \quad Y_{1}^{3}=0, \quad|\eta| Y_{1}^{5}+b_{01}^{4} Y_{0}^{1}=0 ;$

(2) $\quad|\eta| Y_{2}^{2}+\cdots-\frac{D_{0 *}^{2} Y_{0}^{1}}{|\eta|}=0 ; \quad|\eta| Y_{2}^{3}-\frac{D_{0}^{2} Y_{0}^{1}}{|\eta|}=0$;

$b_{04}^{3} Y_{1}^{4}-\frac{b_{02}^{3}}{|\eta|} D_{0} Y_{0}^{1}+\frac{b_{11}^{3}|\eta|-b_{05}^{3} b_{01}^{4}}{|\eta|} Y_{0}^{1}=0$

la $5^{\text {ème }}$ ligne ne donne aucune condition supplémentaire,

$$
\begin{aligned}
& |\eta| Y_{2}^{5}+\cdots+D_{0} Y_{1}^{4}-\frac{D_{0 *}^{2} Y_{0}^{4}}{|\eta|}-\frac{b_{01}^{4}}{|\eta|} D_{*} Y_{0}^{1} \\
& +\frac{\left(b_{11}^{4}|\eta|-D_{*} b_{01}^{4}\right)}{|\eta|} Y_{0}^{1}+\left(D b_{01}^{4}\right)_{*}\left(Y_{0}^{1}\right)=0
\end{aligned}
$$

(3) $|\eta| Y_{3}^{2}+\cdots+\frac{D_{(*)^{2} 0}^{3} Y_{0}^{1}}{|\eta|^{2}}=0, \quad|\eta| Y_{3}^{3}+\cdots+\frac{2 D_{*(0){ }^{2}}^{3} Y_{0}^{1}}{|\eta|^{2}}=0$;

$$
\begin{aligned}
& \left.|\eta| Y_{3}^{5}+\cdots+D_{0} Y_{2}^{4}-\frac{D_{* 0}^{2} Y_{1}^{4}}{|\eta|}+\frac{b_{01}^{4}}{|\eta|^{2}} D_{*}^{2} Y_{0}^{1}+\frac{\left(2 D_{*} b_{01}^{4}-b_{11}^{4}|\eta|\right)}{|\eta|^{2}}\right) D_{*} Y_{0}^{1} \\
& +\left(D b_{11}^{4}\right)_{*}\left(Y_{0}^{1}\right)+\sum_{\left|\alpha^{\prime}\right|=2} \frac{1}{\alpha^{\prime} !} \partial_{\alpha^{\prime}} b_{01}^{4} D^{\alpha^{\prime}} Y_{0}^{1} \\
& \quad-b_{01}^{4} \sum_{\left|\alpha^{\prime}\right|=2} \frac{1}{\alpha^{\prime} !} \partial_{\alpha^{\prime}}|\eta| D^{\alpha^{\prime}} Y_{0}^{1}-2 \sum_{\left|\alpha^{\prime}\right|=2} \frac{1}{\alpha^{\prime} !} \partial_{\alpha^{\prime}}|\eta| D_{\alpha_{1}} b_{01}^{4} D_{\alpha_{2}} Y_{0}^{1} \\
& \quad+\left[\frac{D_{*}^{2} b_{01}^{4}}{|\eta|^{2}}-\frac{D_{*} b_{11}^{4}}{|\eta|}+b_{21}^{4}-\sum_{\left|\alpha^{\prime}\right|=2} \frac{1}{\alpha^{\prime} !} \partial_{\alpha^{\prime}}|\eta|^{2} D^{\alpha^{\prime}} b_{01}^{4}\right] Y_{0}^{1}=0 \\
& b_{04}^{3} Y_{2}^{4}+\cdots+\frac{D_{0}^{3} Y_{0}^{1}}{|\eta|^{2}}-\frac{b_{05}^{3} D_{0} Y_{1}^{4}}{|\eta|}+\frac{b_{02}^{3} D_{0}^{2} Y_{0}^{1}}{|\eta|^{2}}-b_{12}^{3} D_{0} Y_{0}^{1}-\frac{\left(D b_{02}^{3}\right)_{*}}{|\eta|} D_{0} Y_{0}^{1} \\
& \quad+\left(D b_{04}^{3}\right)_{*}\left(Y_{1}^{4}\right)+b_{14}^{3} Y_{1}^{4}+\frac{b_{05}^{3} b_{01}^{4}}{|\eta|^{2}} D_{*} Y_{0}^{1}-\frac{1}{|\eta|}\left[D\left(b_{05}^{3} b_{01}^{4}-b_{11}^{3}|\eta|\right)\right]_{*}\left(Y_{0}^{1}\right) \\
& \quad+\frac{b_{05}^{3}\left(D_{*} b_{01}^{4}-b_{11}^{4}|\eta|\right)}{|\eta|^{2}}-\frac{b_{15}^{3} b_{01}^{4}}{|\eta|}+b_{21}^{3}-\frac{1}{|\eta|}\left(D b_{05}^{3}\right)_{*}\left(b_{01}^{4}\right) Y_{0}^{1}=0 ;
\end{aligned}
$$

compte tenu des conditions $\mathrm{L}$, la $5^{\text {ème }}$ ligne est identiquement vérifiée.

(4) On écrit les $3^{\text {ème }}$ et $5^{\text {ème }}$ lignes:

$$
\begin{aligned}
& D_{0} Y_{3}^{3}+b_{02}^{3} Y_{3}^{2}+b_{04}^{3} Y_{3}^{4}+b_{05}^{3} Y_{3}^{5}+\left(D b_{02}^{3}\right)_{*}\left(Y_{2}^{2}\right)+\left(D b_{04}^{3}\right)_{*}\left(Y_{2}^{4}\right)+\left(D b_{05}^{3}\right)_{*}\left(Y_{2}^{5}\right)_{*} \\
& +b_{11}^{3} Y_{2}^{1}+b_{12}^{3} Y_{2}^{2}+b_{14}^{3} Y_{2}^{4}+b_{15}^{3} Y_{2}^{1}+b_{21}^{3} Y_{1}^{1}+b_{22}^{3} Y_{1}^{2}+b_{24}^{3} Y_{1}^{4}+b_{25}^{3} Y_{1}^{5} \\
& +\left(D b_{11}^{3}\right)_{*}\left(Y_{1}^{1}\right)+\left(D b_{12}^{3}\right)_{*}\left(Y_{1}^{2}\right)+\left(D b_{14}^{3}\right)_{*}\left(Y_{1}^{4}\right)+\left(D b_{15}^{3}\right)_{*}\left(Y_{1}^{5}\right) \\
& \quad+\sum_{\left|\alpha^{\prime}\right|=2} \frac{1}{\alpha^{\prime}}\left[\partial_{\alpha^{\prime}} b_{02}^{3} D^{\alpha^{\prime}} Y_{1}^{2}+\partial_{\alpha^{\prime}} b_{04}^{3} D^{\alpha^{\prime}} Y_{1}^{4}+\partial_{\alpha^{\prime}} b_{05}^{3} D^{\alpha^{\prime}} Y_{1}^{5}\right]+b_{21}^{3} Y_{0}^{1}
\end{aligned}
$$




$$
\begin{aligned}
& +\left(D b_{21}^{3}\right)_{*}\left(Y_{0}^{1}\right)+\sum_{\left|\alpha^{\prime}\right|=2} \frac{1}{\alpha^{\prime} !} \partial_{\alpha^{\prime}} b_{11}^{3} D^{\alpha^{\prime}} Y_{0}^{1}=0 \\
& D_{0} Y_{3}^{5}+b_{04}^{5} Y_{3}^{4}+\left(D b_{04}^{5}\right)_{*}\left(Y_{2}^{4}\right)+b_{11}^{5} Y_{2}^{1}+b_{14}^{5} Y_{2}^{4} \\
& +b_{21}^{5} Y_{1}^{1}+b_{24}^{5} Y_{1}^{4}+\left(D b_{11}^{5}\right)_{*}\left(Y_{1}^{1}\right)+\left(D b_{14}^{5}\right)_{*}\left(Y_{1}^{4}\right) \\
& +\sum_{\left|\alpha^{\prime}\right|=2} \frac{1}{\alpha^{\prime} !} \partial_{\alpha^{\prime}} b_{04}^{5} D^{\alpha^{\prime}} Y_{1}^{4}+b_{31}^{5} Y_{0}^{1}+\left(D b_{21}^{5}\right)_{*}\left(Y_{0}^{1}\right)+\sum_{\left|\alpha^{\prime}\right|=2} \partial_{\alpha^{\prime}} b_{11}^{5} D^{\alpha^{\prime}} Y_{0}^{1}=0
\end{aligned}
$$

On exprime $Y_{3}^{2}, Y_{3}^{5}, Y_{2}^{2}, Y_{2}^{4}, Y_{2}^{5}, Y_{1}^{2}, Y_{1}^{5}, Y_{1}^{4}$ à l'aide des formules précédentes. On écrit que le système en $Y_{3}^{4}$ est compatible. Compte tenu des conditions $\mathrm{L}$, on obtient:

$$
D_{0}^{5} Y_{0}^{1}+W^{I} D_{0}^{4} Y_{0}^{1}+W^{I I} D_{0}^{3} Y_{0}^{1}+W^{I I I} D_{0}^{2} Y_{0}^{1}+W^{I V} D_{0} Y_{0}^{1}+W^{V} Y_{0}^{1}=0
$$

c'est-à-dire l'équation de propagation différentielle ordinaire le long des bicaractéristiques; il suffit alors de déterminer les conditions initiales. On impose:

$$
Y_{0}\left|=0 ; \quad Y_{1}\right|=0 ; \quad Y_{2}\left|=0 ; \quad Y_{3}\right|=I ; \quad \ldots ;
$$

on obtient:

$$
\begin{aligned}
& Y_{0}^{1}\left|=0 ; \quad D_{0} Y_{0}^{1}\right|=0 ; \quad D_{0}^{2} Y_{0}^{1}\left|=0 ; \quad D_{0}^{3} Y_{0}^{1}\right|=0 ;\left.\quad D_{0}^{2} Y_{1}^{1}|=| \eta\right|^{2} I^{3}, \\
& D_{0}^{4} Y_{0}^{1}\left|-\frac{b_{02}^{3}}{b_{04}^{3}}\right||\eta|^{3} I^{3}=|\eta|^{3} I^{5},
\end{aligned}
$$

d'où $D_{0}^{4} Y_{0}^{1} \mid$ est déterminé et par suite $Y_{0}^{1}$ et les $Y$.

b) $b_{01}^{5} \neq 0, b_{04}^{3}=0$. On obtient une équation différentielle d'ordre 5 en $Y_{0}^{4}$ et on détermine comme en a) les conditions initiales.

2) $b_{04}^{3}=b_{01}^{5}=0 . \quad Y_{k}$ est d'ordre $2-k$.

On a:

(0) $Y_{0}^{2}=Y_{0}^{3}=Y_{0}^{5}=0$;

(1) $|\eta| Y_{1}^{2}+D_{0} Y_{0}^{1}=0, \quad Y_{1}^{3}=0 ; \quad|\eta| Y_{1}^{5}+D_{0} Y_{0}^{4}+b_{01}^{4} Y_{0}^{1}=0$

(2) $b_{05}^{3} D_{0} Y_{0}^{4}+b_{05}^{3} b_{01}^{4} Y_{0}^{1}-b_{14}^{3}|\eta| Y_{0}^{4}=0$, $D_{0}^{2} Y_{0}^{4}+b_{01}^{4} D_{0} Y_{0}^{1}+\left(D_{0} b_{01}^{4}-b_{11}^{5}|\eta|\right) Y_{0}^{1}-b_{14}^{5}|\eta| Y_{0}^{4}=0$

$Y_{2}^{2}, Y_{2}^{3}, Y_{2}^{5}$ sont obtenus comme dans le cas a).

(3) $b_{05}^{3} D_{0} Y_{1}^{4}+b_{05}^{3} b_{01}^{4} Y_{1}^{1}-b_{14}^{3}|\eta| Y_{1}^{4}-\frac{D_{0}^{3} Y_{0}^{1}}{|\eta|}-\frac{b_{05}^{3} D_{0 *}^{2} Y_{0}^{4}}{|\eta|}-\frac{b_{05}^{3} b_{01}^{4} D_{*} Y_{0}^{1}}{|\eta|}$

$$
+b_{12}^{3} D_{0} Y_{0}^{1}-\frac{b_{05}^{3} D_{*} b_{01}^{4}}{|\eta|} Y_{0}^{1}+b_{05}^{3}\left(D b_{01}^{4}\right)_{*}\left(Y_{0}^{1}\right)+b_{05}^{3} b_{11}^{4} Y_{0}^{1}
$$




$$
\begin{aligned}
& +\left(D b_{05}^{3}\right)_{*}\left(D_{0} Y_{0}^{4}\right)+\left(D b_{05}^{3}\right)_{*}\left(Y_{0}^{1}\right) b_{01}^{4}+\left(D b_{05}^{3}\right)_{*}\left(b_{01}^{4}\right) Y_{0}^{1}+b_{15}^{3} D_{0} Y_{0}^{4} \\
& +b_{15}^{3} b_{01}^{4} Y_{0}^{1}-b_{21}^{3}|\eta| Y_{0}^{1}-b_{24}^{3}|\eta| Y_{0}^{4}-\left(D b_{14}^{3}\right)_{*}\left(Y_{0}^{4}\right)|\eta|=0 \\
& D_{0}^{2} Y_{1}^{4}+b_{01}^{4} D_{0} Y_{1}^{1}+\left(D_{0} b_{01}^{4}-b_{11}^{5}|\eta|\right) Y_{1}^{1}-b_{14}^{5}|\eta| Y_{1}^{4}-\frac{D_{(0) 2_{*} Y_{0}^{4}}^{|\eta|}}{|\eta|} \\
& \quad-\frac{b_{01}^{4}}{|\eta|} D_{0 *}^{2} Y_{0}^{1}-\frac{D_{0} b_{01}^{4}}{|\eta|} D_{*} Y_{0}^{1}-\frac{D_{*} b_{01}^{4} D_{0} Y_{0}^{1}}{|\eta|}-\frac{D_{0 *}^{2} b_{01}^{4} Y_{0}^{1}}{|\eta|} \\
& +D_{0}\left[\left(D b_{01}^{4}\right)_{*}\left(Y_{0}^{1}\right)\right]+b_{11}^{4} D_{0} Y_{0}^{1}+D_{0} b_{11}^{4} Y_{0}^{1}-b_{21}^{5}|\eta| Y_{0}^{1} \\
& \quad-b_{24}^{5}|\eta| Y_{0}^{4}-|\eta|\left(D b_{11}^{5}\right)_{*}\left(Y_{0}^{1}\right)-|\eta|\left(D b_{14}^{5}\right)_{*}\left(Y_{0}^{4}\right)=0 .
\end{aligned}
$$

On doit distinguer plusieurs cas:

a) $b_{05}^{3} \neq 0 ; b_{1}^{4} \neq 0$ ou $\left[\left(\frac{b_{14}^{3}}{b_{05}^{3}}\right)^{2}|\eta|-b_{14}^{5}+D_{0}\left(\frac{b_{14}^{3}}{b_{05}^{3}}\right)=0\right.$ et $\left.b_{01}^{4}=0\right]$. On reporte $D_{0} Y_{0}^{4}$ de la lère équation (2) dans le deuxième; on constate que celle-ci est identiquement vérifiée. On reporte $D_{0} Y_{1}^{4}$ de la 1ère équation (3) dans le deuxième; on obtient:

$$
D_{0}^{4} Y_{0}^{1}+W D_{0}^{3} Y_{0}^{1}+W^{I} D_{0}^{2} Y_{0}^{1}+W^{I I} D_{0} Y_{0}^{1}+W^{I I I} Y_{0}^{1}+W^{I V} Y_{0}^{4}=0
$$

on a aussi la 1ère équation (2):

$$
D_{0} Y_{0}^{4}+W_{1} Y_{0}^{1}+W_{2} Y_{0}^{4}=0 .
$$

Il suffit donc de déterminer $Y_{0}^{1}\left|, Y_{0}^{4}\right|, D_{0} Y_{0}^{1}\left|, D_{0}^{2} Y_{0}^{1}\right|, D_{0}^{3} Y_{0}^{1} \mid$.

b) $\left[b_{5}^{3} \neq 0, b_{01}^{4}=0\right.$ et $\left.\left(\frac{b_{14}^{3}}{b_{5}^{3}}\right)^{2}|\eta|-b_{14}^{5}+D_{0}\left(\frac{b_{14}^{3}}{b_{05}^{3}}\right) \neq 0\right]$ ou $\left[b_{05}^{3}=0, b_{14}^{3} \neq 0\right]$.

Le système (2) implique: $Y_{0}^{4}=0$; le système (3) donne:

$$
\begin{aligned}
& D_{0}^{2} Y_{1}^{4}+W D_{0} Y_{1}^{4}+W^{I} D_{0}^{2} Y_{0}^{1}+W^{I I} D_{0} Y_{0}^{1}+W^{I I I} Y_{0}^{1}+W^{I V} Y_{1}^{4}=0 \\
& D_{0}^{3} Y_{0}^{1}+W_{1} D_{0} Y_{1}^{4}+W_{2} D_{0} Y_{0}^{1}+W_{3} Y_{0}^{1}+W_{4} Y_{1}^{4}=0
\end{aligned}
$$

il suffit donc de déterminer $Y_{0}^{1}\left|, D_{0} Y_{0}^{1}\right|, D_{0}^{2} Y_{0}^{1}\left|, Y_{1}^{4}\right|, D_{0} Y_{1}^{4} \mid$.

c) $b_{05}^{3}=0, b_{14}^{3}=0$. On obtient:

$$
\begin{aligned}
& D_{0}^{2} Y_{0}^{4}+b_{1}^{4} D_{0} Y_{0}^{1}+\left(D_{0} b_{01}^{4}-b_{11}^{5}|\eta|\right) Y_{0}^{1}-b_{14}^{5} Y_{0}^{4}=0 \\
& -\frac{D_{0}^{3} Y_{0}^{1}}{|\eta|}+b_{12}^{3} D_{0} Y_{0}^{1}+b_{15}^{3} D_{0} Y_{0}^{4}+b_{15}^{3} b_{01}^{4} Y_{0}^{1}-b_{21}^{3}|\eta| Y_{0}^{1}-b_{24}^{3}|\eta| Y_{0}^{4}=0
\end{aligned}
$$

Il reste à déterminer: $Y_{0}^{1}\left|, D_{0} Y_{0}^{1}\right|, D_{0}^{2} Y_{0}^{1}\left|, Y_{1}^{4}\right|$.

On impose dans tous les cas:

$$
Y_{0}\left|=0 ; \quad Y_{1}\right|=0 ; \quad Y_{2} \mid=I ; \quad \cdots
$$

et on détermine tous les coefficients. 


\section{§4. Étude du type $(3,1,1)$}

Les conditions $(\mathrm{LG})_{d}$ s'écrivent:

$$
h=\left(\begin{array}{ccccc}
D_{0} & \left|D^{\prime}\right| & 0 & 0 & 0 \\
0 & D_{0} & \left|D^{\prime}\right| & 0 & 0 \\
b_{1}^{3} & b_{2}^{3} & D_{0} & b_{4}^{3} & b_{5}^{3} \\
b_{1}^{4} & 0 & 0 & D_{0} & b_{5}^{4} \\
b_{1}^{5} & 0 & 0 & b_{4}^{5} & D_{0}
\end{array}\right)
$$

$(\mathrm{LG})_{3 / 2}: b_{01}^{3} \neq 0$

$(\mathrm{LG})_{2}: b_{01}^{3}=0 ; \quad b_{02}^{3} \neq 0$ ou $b_{04}^{3} b_{01}^{4}+b_{05}^{3} b_{01}^{5} \neq 0$

$(\mathrm{LG})_{5 / 2}: b_{01}^{3}=0 ; \quad b_{02}^{3}=0 ; \quad b_{04}^{3} b_{01}^{4}+b_{05}^{3} b_{01}^{5}=0$;

$b_{04}^{3}\left(b_{05}^{4} b_{01}^{5}+D_{0} b_{01}^{4}\right)+b_{05}^{3}\left(b_{04}^{5} b_{01}^{4}+D_{0} b_{01}^{5}\right) \neq 0$

$(\mathrm{LG})_{3}: b_{01}^{3}=0 ; \quad b_{02}^{3}=0 ; \quad b_{04}^{3} b_{01}^{4}+b_{05}^{3} b_{01}^{5}=0$;

$\left(b_{05}^{3} b_{04}^{5}-D_{0} b_{04}^{3}\right) b_{01}^{4}+\left(b_{04}^{3} b_{05}^{4}-D_{0} b_{05}^{3}\right) b_{01}^{5}=0 ; \quad b_{11}^{3} \neq 0$.

On remarque que si le déterminant:

$$
\delta=\operatorname{dét}\left(\begin{array}{cc}
b_{04}^{3} & b_{05}^{3} \\
b_{05}^{3} b_{04}^{5}-D_{0} b_{04}^{3} & b_{04}^{3} b_{05}^{4}-D_{0} b_{05}^{3}
\end{array}\right) \neq 0,
$$

on a: $b_{01}^{4}=b_{01}^{5}=0$.

$$
\begin{aligned}
(\mathrm{LG})_{4}: & b_{01}^{3}=0 ; \quad b_{02}^{3}=0 ; \quad b_{04}^{3} b_{01}^{4}+b_{05}^{3} b_{01}^{5}=0 ; \\
& \left(b_{05}^{3} b_{04}^{5}-D_{0} b_{04}^{3}\right) b_{01}^{4}+\left(b_{04}^{3} b_{05}^{4}-D_{0} b_{05}^{3}\right) b_{01}^{5}=0, \quad b_{11}^{3}=0 ; \\
& C_{4} \equiv b_{04}^{3} b_{11}^{4}+b_{05}^{3} b_{11}^{5}+b_{14}^{3} b_{01}^{4}+b_{15}^{3} b_{01}^{5}+\left(D b_{04}^{3}\right)\left(b_{01}^{4}\right)+\left(D b_{05}^{3}\right)_{*}\left(b_{01}^{5}\right) \neq 0 .
\end{aligned}
$$

On remarque que, si $\delta \neq 0$, on a: $C_{4}=b_{04}^{3} b_{11}^{4}+b_{05}^{3} b_{11}^{5}$.

$$
\begin{aligned}
& (\mathrm{LG})_{5}: b_{01}^{3}=0 ; \quad b_{02}^{3}=0 ; \quad b_{04}^{3} b_{01}^{4}+b_{05}^{3} b_{01}^{5}=0 ; \\
& \left(b_{05}^{3} b_{04}^{5}-D_{0} b_{04}^{3}\right) b_{01}^{4}+\left(b_{04}^{3} b_{05}^{4}-D_{0} b_{05}^{3}\right) b_{01}^{5}=0, \quad b_{11}^{3}=0 ; \quad C_{4}=0 ; \\
& C_{5} \equiv \\
& \quad b_{04}^{3}\left[b_{15}^{4} b_{01}^{5}+b_{05}^{4} b_{11}^{5}+D_{0} b_{11}^{4}+\left(D b_{05}^{4}\right)_{*}\left(b_{01}^{5}\right)\right] \\
& \quad+b_{05}^{3}\left[b_{14}^{5} b_{01}^{4}+b_{04}^{5} b_{11}^{4}+D_{0} b_{11}^{5}+\left(D b_{04}^{5}\right)_{*}\left(b_{01}^{4}\right)\right] \\
& \quad+b_{14}^{3}\left(b_{05}^{4} b_{01}^{5}+D_{0} b_{01}^{4}\right)+b_{15}^{3}\left(b_{04}^{5} b_{01}^{4}+D_{0} b_{01}^{5}\right) \\
& \\
& +\left(D b_{04}^{3}\right)_{*}\left(b_{05}^{4} b_{01}^{5}+D_{0} b_{01}^{4}\right)+\left(D b_{05}^{3}\right)_{*}\left(b_{04}^{5} b_{01}^{4}+D_{0} b_{01}^{5}\right) \neq 0
\end{aligned}
$$

Si $\delta \neq 0$, on a: $b_{11}^{4}=0, b_{11}^{5}=0$.

\subsection{Cas $(\mathrm{LG})_{3 / 2}$.}

$$
f=\sum_{1 \leq t<3} f_{1}^{t}+f_{2}
$$




$$
f_{1}^{t}=e^{\psi_{1}^{t}+\psi_{2}^{t}}\left[Y_{0}^{t}+\cdots+Y_{k}^{t}+\cdots\right]
$$

$\left(D_{0} \psi_{1}^{t}\right)^{3}=-|\eta|^{2} b_{01}^{3} ; \psi_{1}^{t}$ est d'ordre $2 / 3 ;$

$$
D_{0} \psi_{2}^{t}=\frac{2}{3} \frac{D_{0} \psi_{1}^{t} D_{*} \psi_{1}^{t}}{|\eta|}-\frac{b_{02}^{3}}{3 b_{01}^{3}} \frac{\left(D_{0} \psi_{1}^{t}\right)^{2}}{|\eta|}-\frac{1}{3 b_{01}^{3}}\left(D b_{01}^{3}\right)_{*}\left(\psi_{1}^{t}\right) D_{0} \psi_{1}^{t}
$$

$\psi_{2}^{t}$ est d'ordre $1 / 3 ; Y_{k}^{t}$ est d'ordre: $\frac{2-k}{3}$;

$$
f_{2}=Z_{0}+\cdots+Z_{k}+\cdots
$$

$Z_{k}$ est d'ordre $-k / 3$.

Les systèmes en $Y$ et $Z$ sont ceux du $\S 1.3$ b):

On résume fortement le calcul des $Y$ et $\operatorname{des} Z$
(0) $Y_{0}^{2}=Y_{0}^{3}=0$;
(1) $Y_{0}^{4}=Y_{0}^{5}=0$;

(3) le choix de $D_{0} \psi_{1}$ laisse $Y_{0}^{1}$ libre; (4) le choix de $D_{0} \psi_{2}$ laisse $Y_{0}^{1}$ libre;

(5) $3\left(D_{0} \psi_{1}\right)^{2} D_{0} Y_{0}^{1}+W Y_{0}^{1}=0$.
(0) $Z_{0}^{2}=Z_{0}^{3}=0 ; \quad \ldots$;
(3) $b_{01}^{3} Z_{0}^{1}+b_{04}^{3} Z_{0}^{4}+b_{05}^{3} Z_{0}^{5}=0$;
$D_{0} Z_{0}^{4}+b_{01}^{4} Z_{0}^{1}+b_{05}^{4} Z_{0}^{5}=0$
$D_{0} Z_{0}^{5}+b_{01}^{5} Z_{0}^{1}+b_{04}^{5} Z_{0}^{4}=0$

$Z_{0}^{1}$ s'exprime à l'aide de $Z_{0}^{4}$ et $Z_{0}^{5}$ et on a un système différentiel ordinaire d'ordre 1 en $Z_{0}^{4}$ et $Z_{0}^{5}$. On impose:

$$
\sum_{t} Y_{0}^{t}\left|=\sum_{t} Y_{1}^{t}\right|=0 ; \quad \sum_{t} Y_{2}^{t}\left|+Z_{0}\right|=I ; \quad \ldots ;
$$

on obtient:

$$
\sum_{t} Y_{0}^{t 1}\left|=0 ; \quad \sum_{t} D_{0} \psi_{1}^{t} Y_{0}^{t 1}\right|=0 ;\left.\quad \sum_{t}\left(D_{0} \psi_{1}^{t}\right)^{2} Y_{0}^{t 1}|=| \eta\right|^{2} I^{3}
$$

d'où les $Y_{0}^{t 1} \mid$ on a aussi:

$$
Z_{0}^{4}\left|=I^{4}+\frac{b_{01}^{4} \mid}{b_{01}^{3} \mid} I^{3} ; \quad Z_{0}^{5}\right|=I^{5}+\frac{b_{01}^{5} \mid}{b_{01}^{3} \mid} I^{3}
$$

d'où les $Y_{0}^{t 1}, Z_{0}^{4}, Z_{0}^{5}$ et tous les coefficients.

\subsection{Cas (LG) $)_{2}$.}

1) $b_{04}^{3} b_{01}^{4}+b_{05}^{3} b_{01}^{5} \neq 0$. 
1a) On suppose de plus: $\left(b_{02}^{3}\right)^{2}+4\left(b_{04}^{3} b_{01}^{4}+b_{05}^{3} b_{01}^{5}\right) \neq 0$.

$$
\begin{aligned}
f & =\sum_{1 \leq t \leq 4} f_{1}^{t}+f_{2} ; \\
f_{1}^{t} & =e^{\psi^{t}}\left[Y_{0}^{t}+\cdots+Y_{k}^{t}+\cdots\right] ;
\end{aligned}
$$

$D_{0} \psi^{t}$ est l'une des 4 racines distinctes non nulles de l'équation:

$$
\left(D_{0} \psi\right)^{4}-b_{02}^{3}|\eta|\left(D_{0} \psi\right)^{2}-\left(b_{04}^{3} b_{01}^{4}+b_{05}^{3} b_{01}^{5}\right)|\eta|^{2}=0,
$$

$\psi^{t}$ est d'ordre $1 / 2 ; Y_{k}^{t}$ est d'ordre $\frac{2-k}{2}$

$$
f_{2}=Z_{0}+\cdots+Z_{k}+\cdots ;
$$

$Z_{k}$ est d'ordre $-\frac{k}{2}$.

Les systèmes en $Y$ et $Z$ sont ceux du $\S 1.51 \mathrm{a})$. On obtient pour $Y$ :

$$
\text { (0) } Y_{0}^{2}=Y_{0}^{3}=0 ; \quad \text { (1) } Y_{0}^{4}=Y_{0}^{5}=0 ;
$$

(3) laisse $Y_{0}^{1}$ libre compte tenu du choix de $D_{0} \psi$; (4) donne:

$$
2\left(D_{0} \psi\right)^{2}\left(2\left(D_{0} \psi\right)^{2}-b_{02}^{3}\right) D_{0} Y_{0}^{1}+|\eta| C_{3} Y_{0}^{1}=0 .
$$

Pour $Z$ :

$$
\begin{array}{ll}
Z_{0}^{2}=Z_{0}^{3}=0 ; \quad \ldots ; & \text { (2) } \quad b_{04}^{3} Z_{0}^{4}+b_{05}^{3} Z_{0}^{5}=0 \\
D_{0} Z_{0}^{4}+b_{01}^{4} Z_{0}^{1}+b_{05}^{4} Z_{0}^{5}=0, & D_{0} Z_{0}^{5}+b_{01}^{5} Z_{0}^{1}+b_{04}^{5} Z_{0}^{4}=0
\end{array}
$$

on a $b_{04}^{3}$ ou $b_{05}^{3} \neq 0$ par hypothèse; on en déduit:

$$
\left(b_{04}^{3} b_{01}^{4}+b_{05}^{3} b_{01}^{5}\right) Z_{0}^{1}+\left(b_{05}^{3} b_{04}^{5}-D_{0} b_{04}^{3}\right) Z_{0}^{4}+\left(b_{04}^{3} b_{05}^{4}-D_{0} b_{05}^{3}\right) Z_{0}^{5}=0 ;
$$

$Z_{0}^{1}$ s'exprime à l'aide de $Z_{0}^{4}$ et $Z_{0}^{5}$; selon que $b_{04}^{3}$ ou $b_{05}^{3} \neq 0$, on exprime $Z_{0}^{4}$ à l'aide de $Z_{0}^{5}$ ou inversement et on obtient:

$$
D_{0} Z_{0}^{5}+W Z_{0}^{5}=0 \quad\left(\text { resp. } D_{0} Z_{0}^{4}+W Z_{0}^{4}\right)=0 .
$$

On impose les conditions initiales:

$$
\sum_{t} Y_{0}^{t}\left|=\sum_{t} Y_{1}^{t}\right|=0 ; \quad \sum_{t} Y_{2}^{t}\left|+Z_{0}\right|=I ; \quad \ldots ;
$$

on doit préciser les $D_{0} \psi^{t}$; si $1 \leq t \leq 2$ :

$$
\left(D_{0} \psi^{t}\right)^{2}=\frac{b_{02}^{3}+\sqrt{\left(b_{02}^{3}\right)^{2}+4\left(b_{04}^{3} b_{01}^{4}+b_{05}^{3} b_{01}^{5}\right)}}{2}|\eta|
$$

si $3 \leq t \leq 4$, on met le signe - devant le discriminant; on obtient alors:

$$
\text { (0) } \quad \sum_{t} Y_{0}^{t 1} \mid=0 ; \quad \text { (1) } \quad \sum_{1 \leq t \leq 2} D_{0} \psi^{t} Y_{0}^{t 1}\left|=0 ; \quad \sum_{3 \leq t \leq 4} D_{0} \psi^{t} Y_{1}^{t}\right|=0 \text {; }
$$

(2): compte tenu de (0), on a $\sum_{1 \leq t \leq 2} Y_{0}^{t 1}\left|-\sum_{3 \leq t \leq 4} Y_{0}^{t 1}\right|$ en fonction de $I^{3}$ (et du 
discriminant), d'où $\sum_{1 \leq t \leq 2} Y_{0}^{t 1} \mid$ et $\sum_{3 \leq t \leq 4} Y_{0}^{t 1} \mid$ en fonction de $I^{3}$, d'où tous les $Y_{0}^{t 1} \mid$; on a alors: $Z_{0}^{4} \mid$ et $Z_{0}^{5} \mid$ en fonction de $I^{2}, I^{3}, I^{4}$ et $I^{5}$ et tous les coefficients.

1b) $\left(b_{02}^{3}\right)^{2}+4\left(b_{04}^{3} b_{01}^{4}+b_{05}^{3} b_{01}^{5}\right)=0$.

$$
\left(D_{0} \psi^{t}\right)^{2}=\frac{b_{02}^{3}|\eta|}{2}
$$

$\psi^{t}$ est d'ordre $1 / 2$; la quantité $C_{3}$ du 1 a) vaut alors:

$$
\begin{array}{r}
C_{3}=\frac{2}{b_{02}^{3}}\left(b_{05}^{3} b_{04}^{5} b_{01}^{4}+b_{04}^{3} b_{05}^{4} b_{01}^{5}+b_{04}^{3} D_{0} b_{01}^{4}+b_{05}^{3} D_{0} b_{01}^{5}\right) \\
-\frac{4}{\left(b_{02}^{3}\right)^{2}}\left(b_{04}^{3} b_{01}^{4}+b_{05}^{3} b_{01}^{5}\right) D_{0} b_{02}^{3}+b_{11}^{3}|\eta|
\end{array}
$$

On suppose dans $1 \mathrm{~b}): C_{3}=0$.

$$
\begin{aligned}
f & =\sum_{1 \leq t \leq 2} f_{1}^{t}+f_{2} ; \\
f_{1}^{t} & =e^{\psi^{t}}\left[Y_{0}^{t}+\cdots+Y_{k}^{t}+\cdots\right]
\end{aligned}
$$

$Y_{k}^{t}$ est d'ordre: $\frac{3-k}{2}$

$$
f_{2}=Z_{0}+\cdots+Z_{k}+\cdots
$$

$Z_{k}$ est d'ordre $-\frac{k}{2}$.

On reprend les calculs du 1a).

En $Y,(4)$ est identiquement vérifiée: (5) donne:

$$
D_{0}^{2} Y_{0}^{1}+W D_{0} Y_{0}^{1}+W^{\prime} Y_{0}^{1}=0 .
$$

En $Z$, les calculs sont les mêmes; on doit déterminer $Z_{0}^{4} \mid$ ou $Z_{0}^{5} \mid$.

On impose les conditions initiales

$$
\sum_{t} Y_{0}^{t}\left|=\sum_{t} Y_{1}^{t}\right|=0=\sum_{t} Y_{2}^{t}\left|=0 ; \quad \sum_{t} Y_{3}^{t}\right|+Z_{0} \mid=I ; \quad \cdots
$$

(0) et (1) donnent $Y_{0}^{t 1} \mid=0 ;(2)$ donne $\sum_{t} D_{0} Y_{0}^{t 1} \mid=0$;

$$
\text { (3) }\left.2 \sum_{t} D_{0} \psi^{t} D_{0} Y_{0}^{t 1}|=| \eta\right|^{2} I^{3}
$$

d'où les $D_{0} Y_{0}^{t 1} \mid$; on obtient $Z_{0}^{4} \mid$ ou $Z_{0}^{5} \mid$ à l'aide de $I^{2}, I^{3}, I^{4}, I^{5}$. 
1c) $\left(b_{02}^{3}\right)^{2}+4\left(b_{04}^{3} b_{01}^{4}+b_{05}^{3} b_{01}^{5}\right)=0, C_{3} \neq 0$.

$$
\begin{aligned}
f & =\sum_{\substack{1 \leq t \leq 2 \\
1 \leq t^{\prime} \leq 2}} f_{1}^{t t^{\prime}}+f_{2} ; \\
f_{1}^{t t^{\prime}} & =e^{\psi_{1}^{t}+\psi_{1}^{t t^{\prime}}}\left[Y_{0}^{t t^{\prime}}+\cdots+Y_{k}^{t t^{\prime}}+\cdots\right]
\end{aligned}
$$

$\left(D_{0} \psi_{1}^{t}\right)^{2}=\frac{b_{02}^{3}|\eta|}{2} ; \psi_{1}^{t}$ est d'ordre $1 / 2 ;\left(D_{0} \psi_{2}^{t t^{\prime}}\right)^{2}=\frac{-C_{3}}{2 b_{02}^{3}} D_{0} \psi_{1}^{t} ; \psi_{2}^{t t^{\prime}}$ est d'ordre $1 / 4 ;$ $Y_{k}^{t t^{\prime}}$ est d'ordre $\frac{5-k}{4}$;

$$
f_{2}=Z_{0}+\cdots+Z_{k}+\cdots
$$

$Z_{k}$ est d'ordre: $\frac{-k}{4}$.

Pour les $Y$, on obtient en résumant fortement:

$$
\text { (0) } Y_{0}^{2}=Y_{0}^{3}=0 ; \quad \ldots ; \quad(2) \quad Y_{0}^{4}=Y_{0}^{5}=0 ; \quad \ldots ;
$$

(6) laisse $Y_{0}^{1}$ libre par le choix de $D_{0} \psi_{1} ; \ldots ;(8)$ laisse $Y_{0}^{1}$ libre par le choix de $D_{0} \psi_{2}$

$$
\text { (9) } \quad D_{0} Y_{0}^{1}+W Y_{0}^{1}=0 \text {. }
$$

Pour les $Z$, on retrouve les équations du cas 1a). Il reste à déterminer $Z_{0}^{4} \mid$ ou $Z_{0}^{5} \mid$. On impose les conditions initiales:

$$
\begin{aligned}
& \sum_{t t^{\prime}} Y_{0}^{t t^{\prime}}\left|=\sum_{t t^{\prime}} Y_{1}^{t t^{\prime}}\right|=\sum_{t t^{\prime}} Y_{2}^{t t^{\prime}}\left|=\sum_{t t^{\prime}} Y_{3}^{t t^{\prime}}\right|=\sum_{t t^{\prime}} Y_{4}^{t t^{\prime}} \mid=0 \\
& \sum_{t t^{\prime}} Y_{5}^{t t^{\prime}}\left|+Z_{0}\right|=I ; \quad \cdots
\end{aligned}
$$

On obtient:
(0) $\sum_{t t^{\prime}} Y_{0}^{t t^{\prime} 1} \mid=0 ; \quad \ldots$;
(3) $\sum_{t t^{\prime}} D_{0} \psi_{2}^{t t^{\prime}} Y_{0}^{t t^{\prime} 1} \mid=0$
(2) $\quad \sum_{t t^{\prime}} D_{0} \psi_{1}^{t} Y_{0}^{t t^{\prime} 1} \mid=0$;
(4) $\ldots ;$; (5) $\left.2 \sum_{t t^{\prime}} D_{0} \psi_{1}^{t} D_{0} \psi_{2}^{t t^{\prime}} Y_{0}^{t t^{\prime} 1}|=| \eta\right|^{2} I^{3} ; \ldots$

on en déduit, comme au $1.51 \mathrm{c})$ les valeurs des $Y_{0}^{t t^{\prime} 1} \mid$ et par suite les $Y_{0}^{t t^{\prime}}$ et les $Y_{k}^{t t^{\prime}}$; ensuite, on a $Z_{0}^{4} \mid$ ou $Z_{0}^{5} \mid$ à l'aide de $I^{2}, I^{3}, I^{4}$ et $I^{5}$ et les $Z_{k}$.

2) $b_{04}^{3} b_{01}^{4}+b_{05}^{3} b_{01}^{5}=0, b_{02}^{3} \neq 0$. 
a) $C_{3}^{\prime} \equiv\left(b_{05}^{3} b_{04}^{5} b_{01}^{4}+b_{05}^{3} b_{05}^{4} b_{01}^{5}+b_{04}^{3} D_{0} b_{01}^{4}+b_{05}^{3} D_{0} b_{01}^{5}\right) \neq 0$.

$$
\begin{aligned}
f & =\sum_{1 \leq t \leq 2} f_{1}^{t}+\sum_{1 \leq t^{\prime} \leq 3} f_{2}^{t^{\prime}} ; \\
f_{1}^{t} & =e^{\psi^{t}}\left[Y_{0}^{t}+\cdots+Y_{k}^{t}+\cdots\right] ;
\end{aligned}
$$

$\left(D_{0} \psi^{t}\right)^{2}=b_{02}^{3}|\eta| ; \psi^{t}$ est d'ordre $1 / 2 ; Y_{k}^{t}$ est d'ordre: $\frac{6-k}{6}$;

$$
f_{2}^{t^{\prime}}=e^{\chi^{t^{\prime}}}\left[Z_{0}^{t^{\prime}}+\cdots+Z_{k}^{t^{\prime}}+\cdots\right]
$$

$\left(D_{0} \chi^{t^{\prime}}\right)^{3}=\frac{C_{3}^{\prime}}{b_{02}^{3}}|\eta| ; \chi^{t^{\prime}}$ est d'ordre $1 / 3 ; Z_{k}^{t^{\prime}}$ est d'ordre: $\frac{6-k}{6}$.

Pour $Y$, on a:
(0) $Y_{0}^{2}=Y_{0}^{3}=0$
(3) $Y_{0}^{4}=Y_{0}^{5}=0 ; \quad \ldots$;

(9) le choix de $D_{0} \psi_{1}$ laisse $Y_{0}^{1}$ libre; (12) on obtient:

$$
2\left(D_{0} \psi\right)^{2} D_{0} Y_{0}^{1}-b_{02}^{3} D_{0} Y_{0}^{1}+W Y_{0}^{1}=0 .
$$

Pour $Z$, on a:

$$
\text { (0) } Z_{0}^{2}=Z_{0}^{3}=0 ; \quad \ldots ; \quad(4) \quad Z_{0}^{4}=Z_{0}^{5}=0 ; \quad \ldots ;
$$

(10) le choix de $D_{0} \chi$ laisse $Z_{0}^{1}$ libre; ...;

$$
\text { (12) } 3 b_{02}^{3}\left(D_{0} \chi\right)^{3} D_{0} Z_{0}^{1}+W Z_{0}^{1}=0 \text {. }
$$

On impose les conditions initiales:

$$
\sum_{t} Y_{0}^{t}\left|+\sum_{t^{\prime}} Z_{0}^{t^{\prime}}\right|=\cdots=\sum_{t} Y_{5}^{t}\left|+\sum Z_{5}^{t^{\prime}}\right|=0 ; \quad \sum_{t} Y_{6}^{t}\left|+\sum Z_{6}^{t^{\prime}}\right|=I ; \quad \ldots ;
$$

on en déduit aisément les conditions initiales et tous les coefficients.

b) $C_{3}^{\prime}=0$.

$$
\begin{aligned}
f & =\sum_{1 \leq t \leq 2} f_{1}^{t}+f_{2} ; \\
f_{1}^{t} & =e^{\psi_{1}^{t}}\left[Y_{0}^{t}+\cdots+Y_{k}^{t}+\cdots\right] ;
\end{aligned}
$$

$\left(D_{0} \psi_{1}^{t}\right)^{2}=b_{02}^{3}|\eta| ; \psi_{1}^{t}$ est d'ordre $1 / 2 ; Y_{k}^{t}$ est d'ordre $\frac{2-k}{2}$;

$$
f_{2}=Z_{0}^{t^{\prime}}+\cdots+Z_{k}^{t^{\prime}}+\cdots
$$

$Z_{k}^{t^{\prime}}$ est d'ordre: $\frac{2-k}{2}$.

Pour les $Y$, on reprend le calcul de 1a) et on obtient:

$$
D_{0} Y_{0}^{1}+W Y_{0}^{1}=0 \text {. }
$$

Pour les $Z$, on a:

$$
\begin{array}{ll}
\text { (0) } \quad Z_{0}^{2}=Z_{0}^{3}=0 ; \quad(1) \quad \ldots ; \quad(2) \quad b_{04}^{3} Z_{0}^{4}+b_{05}^{3} Z_{0}^{5}=0 \\
& D_{0} Z_{0}^{4}+b_{01}^{4} Z_{0}^{1}+b_{05}^{4} Z_{0}^{5}=0, \quad D_{0} Z_{0}^{5}+b_{01}^{5} Z_{0}^{1}+b_{04}^{5} Z_{0}^{4}=0 .
\end{array}
$$

On doit distinguer plusieurs cas: 
i) $\delta \neq 0$. Alors: $Z_{0}^{4}=Z_{0}^{5}=0$; de plus on déduit de $C_{3}^{\prime}=0$ que $b_{01}^{4}=0=b_{01}^{5}$; $Z_{0}^{1}$ reste libre $(3) \ldots ;(4)$ donne:

$$
\begin{aligned}
& -\frac{b_{02}^{3}}{|\eta|} D_{0} Z_{0}^{1}+b_{04}^{3} Z_{2}^{4}+b_{05}^{3} Z_{2}^{5}+b_{11}^{3} Z_{0}^{1}=0 \\
& D_{0} Z_{2}^{4}+b_{05}^{4} Z_{2}^{5}+b_{11}^{4} Z_{0}^{1}=0 \\
& D_{0} Z_{2}^{5}+b_{04}^{5} Z_{2}^{4}+b_{11}^{5} Z_{0}^{1}=0
\end{aligned}
$$

on en déduit des deux dernières:

$$
D_{0}^{2} Z_{0}^{1}+W D_{0} Z_{0}^{1}+\left(b_{04}^{3} b_{05}^{4}-D_{0} b_{05}^{3}\right) Z_{2}^{5}+\left(b_{05}^{3} b_{04}^{5}-D_{0} b_{04}^{3}\right) Z_{2}^{4}+W^{\prime} Z_{0}^{1}=0
$$

d'où:

$$
D_{0}^{3} Z_{0}^{1}+W^{I I} D_{0}^{2} Z_{0}^{1}+W^{I I I} Z_{0}^{1}+W^{I V} Z_{0}^{1}=0
$$

On impose les conditions initiales

$$
\sum_{t} Y_{0}^{t}\left|+Z_{0}\right|=0=\sum_{t} Y_{1}^{t}\left|+Z_{1}\right| ; \quad \sum_{t} Y_{2}^{t}\left|+Z_{2}\right|=I ; \quad \ldots ;
$$

On en déduit:

$$
\begin{aligned}
& \text { (0) } \sum_{t} Y_{0}^{t 1}\left|+Z_{0}^{1}\right|=0 ; \quad \text { (1) } \sum D_{0} \psi^{t} Y_{0}^{t 1} \mid=0 \\
& \text { (2) } b_{02}^{3} \sum_{t} Y_{0}^{t 1} \mid=I^{3}
\end{aligned}
$$

d'où les $Y_{0}^{t 1} \mid$ et $Z_{0}^{1} \mid$; on a aussi:

$$
b_{02}^{3} D_{0} Z_{0}^{1}|=| \eta \mid\left(b_{04}^{3}\left|I^{4}+b_{05}^{3}\right| I^{5}\right)-\frac{\left.b_{11}^{3}|| \eta\right|^{2}}{b_{02}^{3} \mid} I^{3} ;
$$

$D_{0}^{2} Z_{0}^{1} \mid$ s'exprime à l'aide de $I^{3}, I^{4}, I^{5}$ d'où tous les coefficients.

ii) $\delta=0 ; b_{04}^{3}$ ou $b_{05}^{3} \neq 0$. Il reste ( $\mathrm{si} b_{04}^{3} \neq 0$, par exemple):

$$
D_{0} Z_{0}^{5}+W Z_{0}^{1}+W^{I} Z_{0}^{5}=0
$$

$Z_{0}^{4}$ s'exprime à l'aide de $Z_{0}^{5}$.

On a ensuite:

$$
D_{0}^{2} Z_{0}^{1}+W^{I I} D_{0} Z_{0}^{1}+W^{I I I} Z_{0}^{1}+W^{I V} Z_{0}^{5}=0 .
$$

On impose les mêmes conditions initiales et on détermine tous les coefficients. 
iii) $b_{04}^{3}=b_{05}^{3}=0, b_{02}^{3} \neq 0 . \quad$ On a à l'étape (4):

$$
b_{02}^{3} D_{0} Z_{0}^{1}+W Z_{0}^{1}+W^{I} Z_{0}^{4}+W^{I I} Z_{0}^{5}=0 .
$$

On impose les mêmes conditions initiales que dans les cas précédents; on détermine les $Y_{0}^{t 1}\left|, Z_{0}^{1}\right|, Z_{0}^{4} \mid$ et $Z_{0}^{5} \mid$ et tous les coefficients.

4.3. Cas $(\mathbf{L G})_{5 / 2}$. On a: $C_{3}^{\prime} \neq 0$, où $C_{3}^{\prime}$ est le coefficient défini au $\S 4.2 \mathrm{a}$ )

$$
\begin{aligned}
f & =\sum_{1 \leq t \leq 5} f_{1}^{t} \\
f_{1}^{t} & =e^{\psi_{1}^{t}+\psi_{2}^{t}}\left[Y_{0}^{t}+\cdots+Y_{k}^{t}+\cdots\right]
\end{aligned}
$$

$\left(D_{0} \psi_{1}^{t}\right)^{5}=C_{3}^{\prime}|\eta|^{2}, \psi_{1}^{t}$ est d'ordre $2 / 5 ; D_{0} \psi_{2}^{t}=-\frac{b_{11}^{3}}{5 C_{3}^{\prime}}\left(D_{0} \psi_{1}^{t}\right)^{3} ; \psi_{2}^{t}$ est d'ordre $1 / 5$; $Y_{k}^{t}$ est d'ordre: $\frac{6-k}{5}$.

Les systèmes en $Y$ et $Z$ sont ceux du $\S 1.6$. On obtient pour $Y$, en résumant fortement:

$$
\text { (0) } Y_{0}^{2}=Y_{0}^{3}=0 ; \quad \ldots ; \quad(3) \quad Y_{0}^{4}=Y_{0}^{5}=0 ; \quad \ldots
$$

(9) $Y_{0}^{1}$ reste libre, compte tenu du choix de $D_{0} \psi_{1}$; (10) $Y_{0}^{1}$ reste libre, compte tenu du choix de $D_{0} \psi_{2} ;(11)$ on obtient:

$$
5\left(D_{0}, \psi_{1}\right)^{2} D_{0} Y_{0}^{1}+W Y_{0}^{1}=0
$$

On impose les conditions initiales:

$$
\sum_{t} Y_{0}^{t}\left|=\cdots=\sum_{t} Y_{5}^{t}\right|=0 ; \quad \sum_{t} Y_{6}^{t} \mid=I ; \quad \cdots ;
$$

on obtient:

$$
\begin{aligned}
& \sum_{t} Y_{0}^{t 1}\left|=\sum_{t} D_{0} \psi_{1}^{t} Y_{0}^{t 1}\right|=\sum_{t}\left(D_{0} \psi_{1}^{t}\right)^{2} Y_{0}^{t 1}\left|=\sum_{t}\left(D_{0} \psi_{1}^{t}\right)^{3} Y_{0}^{t 1}\right|=0 \\
& \left.\sum_{t}\left(D_{0} \psi_{1}\right)^{4} Y_{0}^{t 1}|=| \eta\right|^{2} I^{3}
\end{aligned}
$$

d'où les $Y_{0}^{t 1} \mid$ et tous les $Y$. 
4.4. Cas $(\mathrm{LG})_{3}$.

$$
\begin{aligned}
f & =\sum_{1 \leq t \leq 3} f_{1}^{t}+f_{2} \\
f_{1}^{t} & =e^{\psi^{t}}\left[Y_{0}^{t}+\cdots+Y_{k}^{t}+\cdots\right]
\end{aligned}
$$

$\left(D_{0} \psi^{t}\right)^{3}=b_{11}^{3}|\eta| ; \psi^{t}$ est d'ordre $1 / 3 ; Y_{k}^{t}$ est d'ordre: $\frac{4-k}{3} ;$

$$
f_{2}=Z_{0}+\cdots+Z_{k}+\cdots
$$

$Z_{k}$ est d'ordre: $\frac{3-k}{3}$.

Les systèmes en $Y$ et $Z$ sont ceux du $§ 1.7$. On obtient:
(0) $Y_{0}^{2}=Y_{0}^{3}=0$;
(1) $\ldots$;
(2) $Y_{0}^{4}=Y_{0}^{5}=0$
(3) (4) (5) $\ldots$;

(6) laisse $Y_{0}^{1}$ libre compte tenu du choix de $D_{0} \psi$; (7) donne:

$$
3\left(D_{0} \psi\right)^{2} D_{0} Y_{0}^{1}+W Y_{0}^{1}=0
$$

On obtient:

$$
\begin{array}{ll}
\text { (0) } & Z_{0}^{2}=Z_{0}^{3}=0 ; \quad(1) \quad(2) \quad \ldots ; \\
\text { (3) } b_{04}^{3} Z_{0}^{4}+b_{05}^{3} Z_{0}^{5}=0, \quad D_{0} Z_{0}^{4}+b_{01}^{4} Z_{0}^{1}+b_{05}^{4} Z_{0}^{5}=0 \\
\\
D_{0} Z_{0}^{5}+b_{01}^{5} Z_{0}^{1}+b_{04}^{5} Z_{0}^{4}=0
\end{array}
$$

ce système est celui rencontré dans le $\S 4.22 \mathrm{~b}$ ); on distingue de même:

i) $\delta \neq 0$. On a alors:

$$
Z_{0}^{4}=Z_{0}^{5}=0 ; \quad b_{01}^{4}=b_{01}^{5}=0,
$$

$Z_{0}^{1}$ reste libre; on a $(4)(5) \ldots ;(6)$ donne:

$$
\begin{aligned}
& b_{04}^{3} Z_{3}^{4}+b_{05}^{3} Z_{3}^{5}+b_{11}^{3} Z_{0}^{1}=0 \\
& D_{0} Z_{3}^{4}+b_{01}^{4} Z_{3}^{1}+b_{05}^{4} Z_{3}^{5}+b_{11}^{4} Z_{0}^{1}=0 \\
& D_{0} Z_{3}^{5}+b_{01}^{5} Z_{0}^{1}+b_{04}^{5} Z_{3}^{4}+b_{11}^{5} Z_{0}^{1}=0
\end{aligned}
$$

d'où l'on déduit:

$$
-b_{11}^{3} D_{0} Z_{0}^{1}+\left(b_{05}^{3} b_{04}^{5}-D_{0} b_{04}^{3}\right) Z_{3}^{4}+\left(b_{04}^{3} b_{05}^{4}-D_{0} b_{05}^{3}\right) Z_{3}^{5}+W Z_{0}^{1}=0
$$

en éliminant $Z_{3}^{4}$ et $Z_{3}^{5}$, on obtient:

$$
D_{0}^{2} Z_{0}^{1}+W^{\prime} Z_{0}^{1}+W^{\prime \prime} Z_{0}^{1}=0 .
$$


On impose les conditions initiales:

$$
\begin{aligned}
& \sum_{t} Y_{0}^{t}\left|=0 ; \quad \sum_{t} Y_{1}^{t}\right|+Z_{0}\left|=0 ; \quad \sum_{t} Y_{2}^{t}\right|+Z_{1} \mid=0 ; \\
& \sum_{t} Y_{3}^{t}\left|+Z_{2}\right|=0 ; \quad \sum_{t} Y_{4}^{t}\left|+Z_{3}\right|=I ; \quad \cdots
\end{aligned}
$$

On en déduit:

$$
\sum_{t} Y_{0}^{t 1}\left|=\sum_{t} D_{0} \psi^{t} Y_{0}^{t 1}\right|=0 ;\left.\quad \sum_{t}\left(D_{0} \psi^{t}\right)^{2} Y_{0}^{t 1}|=| \eta\right|^{2} I^{3}
$$

d'où les $Y_{0}^{t} \mid$; on a enfin $Z_{0}^{1} \mid$ et $D_{0} Z_{0}^{1} \mid$ à partir de $I^{3}, I^{4}, I^{5}$

ii) $\delta=0, b_{04}^{3}$ ou $b_{01}^{3} \neq 0$. Il reste (si $b_{04}^{3} \neq 0$, par exemple):

$$
D_{0} Z_{0}^{5}+W Z_{0}^{1}+W^{\prime} Z_{0}^{5}=0, \quad D_{0} Z_{0}^{1}+W^{\prime \prime} Z_{0}^{1}+W^{\prime \prime \prime} Z_{0}^{5}=0 .
$$

On détermine les données initiales comme précédemment et tous les coefficients.

iii) $b_{04}^{3}=b_{05}^{3}=0 ; b_{11}^{3} \neq 0$.

$$
Z_{0}^{1}=0, \quad D_{0} Z_{0}^{4}+b_{05}^{4} Z_{0}^{5}=0, \quad D_{0} Z_{0}^{5}+b_{04}^{5} Z_{0}^{4}=0 .
$$

On détermine les données initiales comme précédemment.

\subsection{Cas (LG) 4 .}

$$
\begin{aligned}
f & =\sum_{1 \leq t \leq 4} f_{1}^{t}+f_{2} \\
f_{1}^{t} & =e^{\psi^{t}}\left[Y_{0}^{t}+\cdots+Y_{4}^{t}+\cdots\right]
\end{aligned}
$$

$\left(D_{0} \psi^{t}\right)^{4}=C_{4}|\eta| \neq 0, \psi^{t}$ est d'ordre $1 / 4 ; Y_{k}^{t}$ est d'ordre: $\frac{6-k}{4} ;$

$$
f_{2}=Z_{0}+\cdots+Z_{k}+\cdots
$$

$Z_{k}$ est d'ordre: $\frac{4-k}{4}$.

On obtient:
(0) $Y_{0}^{2}=Y_{0}^{3}=0$
(3) $Y_{0}^{4}=Y_{0}^{5}=0 ; \quad \ldots$;

(9) le choix de $D_{0} \psi$ laisse $Y_{0}^{1}$ libre; (10) on a: $D_{0} Y_{0}^{1}+W Y_{0}^{1}=0$.
(0) $Z_{0}^{2}=Z_{0}^{3}=0 ; \quad \ldots$;
(4) $b_{04}^{3} Z_{0}^{4}+b_{05}^{3} Z_{0}^{5}=0$;
$D_{0} Z_{0}^{4}+b_{01}^{4} Z_{0}^{1}+b_{05}^{4} Z_{0}^{5}=0 ;$
$D_{0} Z_{0}^{5}+b_{01}^{5} Z_{0}^{1}+b_{04}^{5} Z_{0}^{4}=0$; 
ce système est celui rencontré dans les cas du $\S 4.22 \mathrm{~b}$ ) et du $\S 4.4$; on distingue de même:

i) $\delta \neq 0 . \quad$ On a alors: $Z_{0}^{4}=Z_{0}^{5}=0, b_{01}^{4}=b_{01}^{5}=0, Z_{0}^{1}$ reste libre; $\ldots$;

$$
\text { (8) } D Z_{0}^{1}+W Z_{0}^{1}=0
$$

par des calculs analogues aux précédents.

On impose:

$$
\begin{aligned}
& \sum_{t} Y_{0}^{t}\left|=\sum_{t} Y_{1}^{t}\right|=0 \\
& \sum_{t} Y_{2}^{t}\left|+Z_{0}\right|=0=\sum_{t} Y_{3}^{t}\left|+Z_{1}\right|=\sum_{t} Y_{4}^{t}\left|+Z_{2}\right|=\sum_{t} Y_{5}^{t}\left|+Z_{3}\right|=0 \\
& \sum_{t} Y_{6}^{t}\left|+Z_{4}\right|=I ; \quad \cdots
\end{aligned}
$$

On en déduit:

$$
\begin{aligned}
& \sum_{t} Y_{0}^{t 1}\left|=\sum_{t} D_{0} \psi^{t} Y_{0}^{t 1}\right|=\sum_{t}\left(D_{0} \psi^{t}\right)^{3} Y_{0}^{t 1} \mid=0 \\
& \left.\sum_{t}\left(D_{0} \psi^{t}\right)^{2} Y_{0}^{t 1}|=| \eta\right|^{2} I^{3}
\end{aligned}
$$

d'où les $Y_{0}^{t 1} \mid$ et $Z_{0}^{1} \mid$ en fonction de $I^{3}, I^{4}, I^{5}$; d'où tous les coefficients.

ii) $\delta=0, b_{04}^{3}$ ou $b_{05}^{3} \neq 0$. Il reste (si $b_{04}^{3} \neq 0$, par exemple):

$$
D_{0} Z_{0}^{5}+W Z_{0}^{5}=0
$$

$Z_{0}^{4}$ et $Z_{0}^{1}$ s'expriment en fonction de $Z_{0}^{5}$.

On impose les mêmes conditions initiales; on obtient:

$$
\begin{aligned}
& \sum_{t} Y_{0}^{t 1}\left|=\sum_{t} D_{0} \psi^{t} Y_{0}^{t 1}\right|=\sum_{t}\left(D_{0} \psi^{t}\right)^{3} Y_{0}^{t 1} \mid=0 \\
& \left.\sum_{t}\left(D_{0} \psi\right)^{2} Y_{0}^{t 1}|=| \eta\right|^{2} I^{3}
\end{aligned}
$$

d'où les $Y_{0}^{t 1} \mid$ et $Z_{0}^{5} \mid$ en fonction de $I^{3}, I^{4}, I^{5}$; d'où tous les coefficients.

iii) $\quad b_{04}^{3}=b_{05}^{3}=0 ; b_{14}^{3} b_{01}^{4}+b_{15}^{3} b_{01}^{5} \neq 0 . \quad b_{14}^{3}$ ou $b_{15}^{3}$ est $\neq 0 ;$ on obtient (si $b_{14}^{3} \neq 0$ par exemple):

$$
D_{0} Z_{0}^{5}+W Z_{0}^{5}=0
$$

On détermine les données initiales comme précédemment. 
4.6. Cas $(\mathbf{L G})_{5}, \quad C_{5} \neq 0$;

$$
\begin{aligned}
f & =\sum_{1 \leq t \leq 5} f^{t} \\
f^{t} & =e^{\psi^{t}}\left[Y_{0}^{t}+\cdots+Y_{k}^{t}+\cdots\right]
\end{aligned}
$$

$\left(D_{0} \psi^{t}\right)^{5}=C_{5}|\eta|^{2} . \psi^{t}$ est d'ordre $1 / 5 ; Y_{k}^{t}$ est d'ordre: $\frac{8-k}{5}$. On obtient:

$$
\text { (0) } Y_{0}^{2}=Y_{0}^{3}=0 ; \quad \ldots ; \quad(4) \quad Y_{0}^{4}=Y_{0}^{5}=0 ; \quad \ldots ;
$$

(12) laisse $Y_{0}^{1}$ libre par le choix de $D_{0} \psi ;(15)$ donne: $D_{0} Y_{0}^{1}+W Y_{0}^{1}=0$.

On impose:

$$
\sum_{t} Y_{0}^{t}\left|=\cdots=\sum_{t} Y_{7}^{t}\right|=0 ; \quad \sum_{t} Y_{8}^{t} \mid=I
$$

En distinguant des cas comme précédemment, on obtient tous les coefficients.

\subsection{Cas L.}

$$
f=Y_{0}+\cdots+Y_{k}+\cdots
$$

$Y_{k}$ est d'ordre $2-k$.

On distingue plusieurs cas

1) $\delta \neq 0$. Dans ce cas, il résulte des conditions L que:

$$
b_{01}^{4}=b_{01}^{5}=b_{11}^{4}=b_{11}^{5}=0 .
$$

On obtient:

$$
\begin{array}{llll}
Y_{0}^{2}=Y_{0}^{3}=0 ; & \text { (1) } & |\eta| Y_{1}^{2}+D_{0} Y_{0}^{1}=0, & Y_{1}^{3}=0, \\
& b_{04}^{3} Y_{0}^{4}+b_{05}^{3} Y_{0}^{5}=0, & D_{0} Y_{0}^{4}+b_{05}^{4} Y_{0}^{5}=0, & D_{0} Y_{0}^{5}+b_{04}^{5} Y_{0}^{4}=0 ;
\end{array}
$$

d'où: $Y_{0}^{4}=Y_{0}^{5}=0$

$$
\begin{array}{ll} 
& |\eta|^{2} Y_{2}^{3}-D_{0}^{2} Y_{0}^{1}=0, \quad b_{04}^{3} Y_{1}^{4}+b_{05}^{3} Y_{1}^{5}=0, \quad D_{0} Y_{1}^{4}+b_{05}^{4} Y_{1}^{5}=0 \\
& D_{0} Y_{1}^{5}+b_{04}^{5} Y_{1}^{4}=0,
\end{array}
$$

d'où: $Y_{1}^{4}=Y_{1}^{5}=0$,

$$
\text { (3) } \begin{array}{ll}
|\eta|^{2}\left(b_{04}^{3} Y_{2}^{4}+b_{05}^{3} Y_{2}^{5}\right)+D_{0}^{3} Y_{0}^{1}+|\eta|^{2} b_{31}^{2} Y_{0}^{1}=0 \\
& D_{0} Y_{2}^{4}+b_{05}^{4} Y_{2}^{5}+b_{21}^{4} Y_{0}^{1}=0, \quad D_{0} Y_{2}^{5}+b_{04}^{5} Y_{2}^{4}+b_{21}^{5} Y_{0}^{1}=0
\end{array}
$$

d'où:

$$
\begin{array}{r}
|\eta|^{2}\left[\left(b_{05}^{3} b_{04}^{5}-D_{0} b_{04}^{3}\right) Y_{2}^{4}+\left(b_{05}^{3} b_{04}^{5}-D_{0} b_{05}^{3}\right) Y_{2}^{5}\right]-D_{0}^{4} Y_{0}^{1} \\
-|\eta|^{2} D_{0}\left(b_{31}^{2} Y_{0}^{1}\right)+|\eta|^{2}\left(b_{04}^{3} b_{21}^{4}+b_{05}^{3} b_{21}^{5}\right) Y_{0}^{1}=0
\end{array}
$$


d'où en déterminant $Y_{2}^{4}$ et $Y_{2}^{5}$ par la 1ère et la $4^{\text {ème }}$ équation et en reportant dans l'une des 2 autres:

$$
b_{04}^{3} D_{0}^{5} Y_{0}^{1}+W D_{0}^{4} Y_{0}^{1}+W^{\prime} D_{0}^{3} Y_{0}^{1}+W^{\prime \prime} D_{0}^{2} Y_{0}^{1}+W^{\prime \prime \prime} D_{0} Y_{0}^{1}+W^{I V} Y_{0}^{1}=0 .
$$

On impose les conditions initiales:

$$
Y_{0}\left|=Y_{1}\right|=0 ; \quad Y_{2} \mid=I
$$

d'où l'on déduit:

$$
\begin{aligned}
& Y_{0}^{1}\left|=D_{0} Y_{0}^{1}\right|=0 ;\left.\quad D_{0}^{2} Y_{0}^{1}|=| \eta\right|^{2} I^{3},\left.\quad D_{0}^{3} Y_{0}^{1}|+| \eta\right|^{2}\left(b_{04}^{3}\left|I^{4}+b_{05}^{3}\right| I^{5}\right)=0 \\
& \left.D_{0}^{4} Y_{0}^{1}|=| \eta\right|^{2}\left[\left(b_{05}^{3} b_{04}^{5}-D_{0} b_{04}^{3}\right)\left|I^{4}+\left(b_{04}^{3} b_{05}^{4}-D_{0} b_{05}^{3}\right)\right| I^{5}\right]
\end{aligned}
$$

d'où les données initiales et tous les coefficients.

2) $\delta=0, b_{04}^{3}$ ou $b_{05}^{3} \neq 0$. On a:

$$
\begin{gathered}
Y_{0}^{2}=Y_{0}^{3}=0 \quad(1) \quad \ldots ; \quad b_{04}^{3} Y_{0}^{4}+b_{05}^{3} Y_{0}^{5}=0 \\
D_{0} Y_{0}^{4}+b_{01}^{4} Y_{0}^{1}+b_{05}^{4} Y_{0}^{5}=0
\end{gathered}
$$

ou

$$
D_{0} Y_{0}^{5}+b_{01}^{5} Y_{0}^{1}+b_{04}^{5} Y_{0}^{4}=0
$$

(2) $b_{04}^{3} Y_{1}^{4}+b_{05}^{3} Y_{1}^{5}+b_{14}^{3} Y_{0}^{4}+b_{15}^{3} Y_{0}^{5}+\left(D b_{04}^{3}\right)_{*}\left(Y_{0}^{4}\right)+\left(D b_{05}^{3}\right)_{*}\left(Y_{0}^{5}\right)=0$

$$
\begin{aligned}
& D_{0} Y_{1}^{4}+b_{01}^{4} Y_{1}^{1}+b_{05}^{4} Y_{1}^{5}+b_{11}^{4} Y_{0}^{1}+b_{15}^{4} Y_{0}^{5}+\left(D b_{01}^{4}\right)_{*}\left(Y_{0}^{1}\right)_{*}+\left(D b_{05}^{4}\right)_{*}\left(Y_{0}^{5}\right)=0 \\
& D_{0} Y_{1}^{5}+b_{01}^{5} Y_{1}^{1}+b_{04}^{5} Y_{1}^{4}+b_{11}^{5} Y_{0}^{1}+b_{14}^{5} Y_{0}^{4}+\left(D b_{01}^{5}\right)_{*}\left(Y_{0}^{1}\right)+\left(D b_{04}^{5}\right)_{*}\left(Y_{0}^{4}\right)=0
\end{aligned}
$$

d'où:

$$
\begin{aligned}
\left(b_{05}^{3} b_{04}^{5}-\right. & \left.D_{0} b_{04}^{3}\right) Y_{1}^{4}+\left(b_{04}^{3} b_{05}^{4}-D_{0} b_{05}^{3}\right) Y_{1}^{5} \\
& +\left[b_{15}^{3} b_{04}^{5}+b_{05}^{3} b_{14}^{5}-D_{0} b_{14}^{3}+\left(D b_{05}^{3}\right)_{*}\left(b_{04}^{5}\right)\right] Y_{0}^{4} \\
& +\left[b_{14}^{3} b_{05}^{4}+b_{04}^{3} b_{15}^{4}-D_{0} b_{15}^{3}+\left(D b_{04}^{3}\right)_{*}\left(b_{05}^{4}\right)\right] Y_{0}^{5} \\
& +\left(D\left(b_{05}^{3} b_{04}^{5}-D_{0} b_{04}^{3}\right)\right)_{*}\left(Y_{0}^{4}\right)+\left(D\left(b_{04}^{3} b_{05}^{4}-D_{0} b_{05}^{3}\right)\right)_{*}\left(Y_{0}^{5}\right)=0
\end{aligned}
$$

les équations en $Y_{1}^{4}$ et $Y_{1}^{5}$ sont compatibles, sans nouvelle condition sur $Y_{0}^{4}$ ou $Y_{0}^{5}$.

(3) On procède de même en $Y_{2}^{4}, Y_{2}^{5}$ et on obtient:

$$
D_{0}^{4} Y_{0}^{1}+W^{\prime} D_{0}^{3} Y_{0}^{1}+W^{\prime \prime} D_{0}^{2} Y_{0}^{1}+W^{I I I} D_{0} Y_{0}^{1}+W^{I V} Y_{0}^{1}+W^{V} Y_{0}^{4}+W^{V I I} Y_{0}^{5}=0
$$


On impose les mêmes conditions aux données initiales et on obtient celles-ci et tous les coefficients.

3) $b_{04}^{3}=b_{05}^{3}=0$, dét $\left(\begin{array}{cc}b_{14}^{3} & b_{15}^{3} \\ b_{15}^{3} b_{04}^{5}-D_{0} b_{14}^{3} & b_{14}^{3} b_{05}^{4}-D_{0} b_{15}^{3}\end{array}\right) \neq 0 . \quad$ On en déduit: $b_{01}^{4}=b_{01}^{5}=0$.

On a alors:

$$
Y_{0}^{4}=Y_{0}^{5}=0
$$

et

$$
D_{0}^{5} Y_{0}^{1}+W D_{0}^{4} Y_{0}^{1}+W^{\prime} D_{0}^{3} Y_{0}^{1}+W^{\prime \prime} D_{0}^{2} Y_{0}^{1}+W^{\prime \prime \prime} Y_{0}^{1}=0
$$

on détermine les données comme précédemment.

4) $b_{04}^{3}=b_{05}^{3}=0$, dét $\left(\begin{array}{cc}b_{14}^{3} & b_{15}^{3} \\ b_{15}^{3} b_{04}^{5}-D_{0} b_{14}^{3} & b_{14}^{3} b_{05}^{4}-D_{0} b_{15}^{3}\end{array}\right)=0 . \quad b_{14}^{3}$ ou $b_{15}^{3} \neq 0$.

On obtient: $b_{14}^{3} Y_{0}^{4}+b_{15}^{3} Y_{0}^{5}=0$

$$
\begin{aligned}
& D_{0} Y_{0}^{4}+b_{01}^{4} Y_{0}^{1}+b_{05}^{4} Y_{0}^{5}=0, \quad D_{0} Y_{0}^{5}+b_{01}^{5} Y_{0}^{1}+b_{04}^{5} Y_{0}^{4}=0 \\
& D_{0}^{4} Y_{0}^{1}+W^{I} D_{0}^{3} Y_{0}^{1}+W^{I I} D_{0}^{2} Y_{0}^{1}+W^{I I I} D_{0} Y_{0}^{1}+W^{I V} Y_{0}^{1}+W^{V} Y_{0}^{4}+W^{V I} Y_{0}^{5}=0
\end{aligned}
$$

on finit comme précédemment.

5) $b_{04}^{3}=b_{05}^{3}=b_{14}^{3}=b_{15}^{3}=0$. On a:

$$
\begin{aligned}
& D_{0} Y_{0}^{4}+b_{01}^{4} Y_{0}^{1}+b_{05}^{4} Y_{0}^{5}=0 \\
& D_{0} Y_{0}^{5}+b_{01}^{5} Y_{0}^{1}+b_{04}^{5} Y_{0}^{4}=0 \\
& D_{0}^{3} Y_{0}^{1}+W Y_{0}^{1}+W^{\prime} Y_{0}^{4}+W^{\prime \prime} Y_{0}^{5}=0:
\end{aligned}
$$

on finit comme précédemment.

§5. Étude du type $(2,2,1)$

$$
h=\left(\begin{array}{ccccc}
D_{0} & \left|D^{\prime}\right| & 0 & 0 & 0 \\
b_{1}^{2} & D_{0} & b_{3}^{2} & b_{4}^{2} & b_{5}^{2} \\
b_{1}^{3} & 0 & D_{0} & \left|D^{\prime}\right| & 0 \\
b_{1}^{4} & 0 & b_{3}^{4} & D_{0} & b_{5}^{4} \\
b_{1}^{5} & 0 & b_{3}^{5} & 0 & D_{0}
\end{array}\right)
$$


Notations. Dans ce cas on note: $C, D, \ldots$ des indices prenant les valeurs 1 ou 3;

$$
\begin{aligned}
& \beta=\left(\beta_{D}^{C}\right)=\left(b_{0 D}^{C+1}\right) ; \quad \beta_{1}=\left(\beta_{1 D}^{C}\right)=\left(b_{1 D}^{C+1}\right) \\
& \beta^{\prime}=\left({\beta^{\prime}}_{D}^{C}\right)=\left(\begin{array}{cc}
0 & 0 \\
b_{01}^{3} & 0
\end{array}\right) ; \beta^{\prime \prime}=\left({\beta^{\prime \prime}}_{D}^{C}\right)=\left(\begin{array}{cc}
0 & b_{04}^{2} \\
0 & 0
\end{array}\right) ; \hat{\beta}=\beta^{\prime}+\beta^{\prime \prime} \\
& \gamma^{\prime}=\left(\gamma^{\prime C}\right)=\left(b_{05}^{C+1}\right) ; \quad \gamma^{\prime \prime}=\left(\gamma_{C}^{\prime \prime 5}\right)=\left(b_{0 C}^{5}\right) ; \quad \gamma=\left(\gamma_{D}^{C}\right)=\left(b_{05}^{C+1} b_{0 D}^{5}\right) \\
& \gamma_{1}^{\prime}=\left(\gamma_{1}^{\prime C}\right)=\left(b_{15}^{C+1}\right) ; \quad \gamma_{1}^{\prime \prime}=\left({\gamma^{\prime \prime}}_{1 C}^{5}\right)=\left(b_{1 C}^{5}\right) ; \quad \gamma_{1}=\left(\gamma_{1 D}^{C}\right)=\left(b_{1 D}^{C+1} b_{1 D}^{5}\right) \text {. }
\end{aligned}
$$

On remarque que: $\gamma^{2}=\gamma \operatorname{Tr} \gamma$ et dét $\gamma=0$, où $\operatorname{Tr} \gamma=\operatorname{Trace} \gamma$. On pose:

$$
\begin{aligned}
& M_{5 / 2} \equiv \beta \gamma \beta \\
& M_{3} \equiv \beta \hat{\beta} \beta+\beta \gamma+\gamma \beta+2 \beta D_{0} \beta \\
& M_{3}^{\prime} \equiv \operatorname{Tr} ; \\
& M_{4} \equiv- \beta \beta^{\prime \prime} \beta^{\prime} \beta+\gamma \hat{\beta} \beta+\beta \hat{\beta} \gamma+\gamma^{2}+\beta \beta_{1} \beta-\beta D_{0} \beta^{\prime} \beta-2\left(D_{0} \beta\right)^{2} \\
&+\beta\left(\hat{\beta} D_{0} \beta+\gamma^{\prime} D_{0} \gamma^{\prime \prime}\right)+\left(-D_{0} \gamma-D_{0} \gamma^{\prime} \gamma^{\prime \prime}+D_{0}^{2} \beta-2 D_{0} \beta \hat{\beta}\right) \beta \\
&+\gamma D_{0} \beta-2 D_{0} \beta \gamma+\beta(D \beta)_{*}(\beta) \\
& M_{4}^{\prime} \equiv \gamma \hat{\beta} \gamma+\gamma D_{0} \gamma-D_{0} \gamma \gamma \\
& M_{5} \equiv \hat{\beta}^{2}(\beta \gamma+\gamma \beta)-\gamma \beta^{\prime \prime} \beta^{\prime} \beta-\beta \beta^{\prime \prime} \beta^{\prime} \gamma+\gamma \hat{\beta} \gamma+\beta \beta_{1} \hat{\beta} \beta+\beta \hat{\beta} \beta_{1} \beta \\
&+\gamma \beta_{1} \beta+\beta \beta_{1} \gamma+\beta\left(\gamma^{\prime} \gamma_{1}^{\prime \prime}+\gamma_{1}^{\prime} \gamma^{\prime \prime}\right) \beta-\beta\left(D_{0} \hat{\beta} \beta^{\prime \prime}-\hat{\beta} D_{0} \beta^{\prime \prime}\right) \beta \\
&+\gamma\left(D_{0} \beta^{\prime \prime} \beta+2 \hat{\beta} D_{0} \beta+D_{0}^{2} \beta+D_{0} \gamma+\gamma^{\prime} D_{0} \gamma^{\prime \prime}\right) \\
&+\left(-\beta D_{0} \beta^{\prime}-2 D_{0} \beta \hat{\beta}+D_{0}^{2} \beta-D_{0} \gamma+D_{0} \gamma^{\prime} \gamma^{\prime \prime}\right) \gamma \\
&+\beta\left[2\left(\hat{\beta}^{2}+\hat{\beta} \beta^{\prime \prime}+\beta_{1}-D_{0} \beta^{\prime}\right) D_{0} \beta+\hat{\beta} D_{0}^{2} \beta+\hat{\beta} D_{0} \gamma\right. \\
&\left.+\hat{\beta}^{\prime} D_{0} \gamma^{\prime \prime}+\gamma^{\prime} D_{0}^{2} \gamma^{\prime \prime}\right] \\
&+\left[2 D_{0} \beta\left(\beta^{\prime \prime} \hat{\beta}-\beta_{1}-D_{0} \beta^{\prime \prime}\right)+D_{0}^{2} \beta \hat{\beta}-D_{0} \gamma \hat{\beta}-D_{0} \gamma^{\prime} \gamma^{\prime \prime} \hat{\beta}+D_{0}^{2} \gamma^{\prime} \gamma^{\prime \prime}\right] \beta \\
&-4 D_{0} \beta \hat{\beta} D_{0} \beta+2\left(D_{0}^{2} \beta D_{0} \beta-D_{0} \beta D_{0}^{2} \beta\right)-4 D_{0} \gamma D_{0} \beta \\
&-2 D_{0} \beta D_{0} \gamma+2 \gamma^{\prime} D_{0} \gamma^{\prime \prime} D_{0} \beta-2 D_{0} \beta \gamma^{\prime} D_{0} \gamma^{\prime \prime}+\beta(D \beta)_{*}\left(\hat{\beta} \beta+\gamma+2 D_{0} \beta\right) \\
&+\left(\beta \hat{\beta}+\gamma-2 D_{0} \beta\right)(D \beta)_{*}(\beta)+\beta(D \gamma)_{*}(\beta)+\beta\left(D \gamma^{\prime}\right)_{*}\left(\gamma^{\prime \prime}\right) \cdot \beta \\
& M_{5}^{\prime} \equiv- \gamma \beta^{\prime \prime} \beta^{\prime} \gamma+\gamma \beta_{1} \gamma-D_{0} \gamma \hat{\beta} \gamma+\gamma D_{0} \beta^{\prime \prime} \gamma+2 \gamma \hat{\beta} D_{0} \gamma \\
&-2 D_{0} \gamma D_{0} \gamma+\gamma D_{0}^{2} \gamma-D_{0} \gamma^{\prime} \gamma^{\prime \prime} \hat{\beta} \gamma+\gamma \hat{\beta} \gamma^{\prime} D_{0} \gamma^{\prime \prime} \\
&-2 D_{0} \gamma^{\prime} \gamma^{\prime \prime} D_{0} \gamma-D_{0} \gamma \gamma^{\prime} D_{0} \gamma^{\prime \prime}+\gamma D_{0} \gamma^{\prime} D_{0} \gamma^{\prime \prime} . \\
&
\end{aligned}
$$

Les conditions $(\mathrm{LG})_{d}$ s'écrivent:

$$
\begin{aligned}
(\mathrm{LG})_{2} & : \beta^{2} \neq 0 \\
(\mathrm{LG})_{5 / 2} & : \beta^{2}=0, \quad M_{5 / 2} \neq 0
\end{aligned}
$$




$$
\begin{gathered}
(\mathrm{LG})_{3}:\left(\beta^{2}=0, \beta \neq 0, M_{5 / 2}=0, M_{3} \neq 0\right) \text { ou }\left(\beta=0, M_{3}^{\prime} \neq 0\right) \\
(\mathrm{LG})_{4}:\left(\beta^{2}=0, \beta \neq 0, M_{3}=0, M_{4} \neq 0\right) \text { ou }\left(\beta=0, M_{3}^{\prime}=0, M_{4}^{\prime} \neq 0\right) \\
(\mathrm{LG})_{5}:\left(\beta^{2}=0, \beta \neq 0, M_{3}=M_{4}=0, M_{5} \neq 0\right) \\
\quad \text { ou }\left(\beta=0, M_{3}^{\prime}=0=M_{4}^{\prime}, M_{5}^{\prime} \neq 0\right) \\
\mathrm{L}:\left(\beta^{2}=0, \beta \neq 0, M_{3}=M_{4}=M_{5}=0\right) \\
\text { ou }\left(\beta=0, M_{3}^{\prime}=M_{4}^{\prime}=M_{5}^{\prime}=0\right) .
\end{gathered}
$$

Il sera commode de les détailler de la façon suivante. On notera: si $\beta \neq 0, \tilde{\beta}$ une matrice équivalente à $\beta: \tilde{\beta}=U^{-1} \beta U$; on notera aussi:

$$
\begin{gathered}
\tilde{\hat{\beta}}=U^{-1} \hat{\beta} U ; \quad \ldots ; \quad \tilde{\gamma}^{\prime \prime}=\gamma^{\prime \prime} U ; \quad \tilde{\gamma}^{\prime}=U^{-1} \gamma^{\prime} ; \quad \tilde{\gamma}=U^{-1} \gamma U ; \quad \ldots \\
\mathcal{C}=U^{-1} D_{0} U ; \quad \mathcal{C}_{\xi}=U^{-1} \frac{\partial U}{\partial \xi^{\prime}} ; \quad \mathcal{C}_{x}=U^{-1} \frac{\partial U}{\partial x^{\prime}} ; \quad \mathcal{C}_{\xi} \cdot \mathcal{C}_{x}=\sum_{j} U^{-1} \frac{\partial U}{\partial \xi_{j}} U^{-1} \frac{\partial U}{\partial x_{j}}
\end{gathered}
$$

$(\mathrm{LG})_{2}$ On distingue les cas suivants:

1i) On suppose dét $\beta \neq 0,(\operatorname{Tr} \beta)^{2}-4 \operatorname{dét} \beta \neq 0$.

Les 2 valeurs propres $\lambda_{1}$ et $\lambda_{2}$ de $\beta$ sont distinctes et non nulles

$$
\beta \sim \tilde{\beta}=\left(\begin{array}{cc}
\lambda_{1} & 0 \\
0 & \lambda_{2}
\end{array}\right), \quad \lambda_{1} \neq \lambda_{2}, \quad \lambda_{1} \neq 0, \quad \lambda_{2} \neq 0 .
$$

1ii) dét $\beta \neq 0,(\operatorname{Tr} \beta)^{2}-4$ dét $\beta=0$. Les 2 valeurs propres sont égales et non nulles; on suppose de plus:

$$
\beta \sim \tilde{\beta}=\left(\begin{array}{ll}
\lambda & 1 \\
0 & \lambda
\end{array}\right), \quad \lambda \neq 0
$$

1iii) dét $\beta \neq 0,(\operatorname{Tr} \beta)^{2}-4$ dét $\beta=0$. Les 2 valeurs propres sont égales et non nulles; de plus:

$$
\beta \sim \tilde{\beta}=\left(\begin{array}{ll}
\lambda & 0 \\
0 & \lambda
\end{array}\right), \quad \lambda \neq 0
$$

2) On suppose dét $\beta=0, \operatorname{Tr} \beta \neq 0$

$$
\beta \sim \tilde{\beta}=\left(\begin{array}{ll}
\lambda & 0 \\
0 & 0
\end{array}\right), \quad \lambda \neq 0 .
$$

$(\mathrm{LG})_{5 / 2}$ On a: $\beta^{2}=0, \beta \neq 0$, d'où:

$$
\beta \sim \tilde{\beta}=\left(\begin{array}{ll}
0 & 1 \\
0 & 0
\end{array}\right)
$$


et $M_{5 / 2} \neq 0$ s'écrit alors: $\tilde{\gamma}_{1}^{3} \neq 0$.

$(\mathrm{LG})_{3}$ :

1) $\operatorname{Si} \beta^{2}=0, \beta \neq 0$, on a encore: $\beta \sim \tilde{\beta}=\left(\begin{array}{ll}0 & 1 \\ 0 & 0\end{array}\right), \tilde{\gamma}_{1}^{3}=0$ et:

$$
C_{3} \equiv \tilde{\hat{\beta}}_{1}^{3}+\tilde{\gamma}_{1}^{1}+\tilde{\gamma}_{3}^{3}+2 \mathcal{C}_{1}^{3} \neq 0
$$

on a remarqué que: $\widetilde{D_{0} \beta}=D_{0} \tilde{\beta}+\mathcal{C} \beta-\beta \mathcal{C}$.

2) Si $\beta=0$, la condition s'écrit: $\operatorname{Tr} \gamma \neq 0$; comme dét $\gamma=0$, on peut réduire $\gamma$ comme on avait réduit $\beta$ dans les cas précédents, on a:

$$
\gamma \sim \tilde{\gamma}=\left(\begin{array}{cc}
\lambda & 0 \\
0 & 0
\end{array}\right)=U^{-1} \gamma U ; \quad \lambda=\operatorname{Tr} \gamma=\operatorname{Tr} \tilde{\gamma}=\tilde{\gamma}^{\prime 1} \tilde{\gamma}_{1}^{\prime \prime} \neq 0 .
$$

$(\mathrm{LG})_{4}$

1) $\operatorname{Si} \beta^{2}=0, \beta \neq 0$, on a encore: $\beta \sim \tilde{\beta}=\left(\begin{array}{ll}0 & 1 \\ 0 & 0\end{array}\right), \tilde{\gamma}_{1}^{3}=0$;

$$
\begin{aligned}
& C_{3}=0 \text {; } \\
& C_{4} \equiv{\tilde{\beta^{\prime \prime}}}_{1}{ }_{1}^{1}\left(\tilde{\gamma}_{1}^{1}-\tilde{\gamma}_{3}^{3}\right)-{\tilde{\beta^{\prime \prime}}}_{3}{ }_{3}^{3}{\tilde{\beta}^{\prime}}_{1}^{3}-\tilde{\gamma}_{3}^{1}\left(\tilde{\gamma}_{1}^{1}+\tilde{\gamma}_{3}^{3}\right)-D_{0}{\tilde{\beta^{\prime}}}_{1}^{3}+{\tilde{\gamma^{\prime}}}^{3} \cdot D_{0} \tilde{\gamma}_{3}^{\prime \prime} \\
& +\tilde{\gamma}^{\prime 1} \cdot D_{0} \tilde{\gamma}_{1}^{\prime \prime}-2 D_{0} \tilde{\gamma}_{1}^{1}+\left(-{\tilde{\beta}^{\prime \prime}}_{1}^{3}-2 \tilde{\gamma}_{3}^{3}\right) \mathcal{C}_{1}^{1}+\left(\tilde{\beta}^{\prime{ }^{1}}{ }_{1}+2 \tilde{\gamma}_{3}^{1}\right) \mathcal{C}_{1}^{3} \\
& -D_{0} \mathcal{C}_{1}^{3}-\mathcal{C}_{1}^{1} \mathcal{C}_{1}^{3}+\beta_{11}^{3}|\eta|+\mathcal{C}_{\xi 1}^{3} \cdot \mathcal{C}_{x 1}^{3} \neq 0 \text {. }
\end{aligned}
$$

2) Si $\beta=0$, on a: $\operatorname{Tr} \gamma=0, M_{4} \neq 0 ; \gamma \sim \tilde{\gamma}=\left(\begin{array}{ll}0 & 1 \\ 0 & 0\end{array}\right)=U^{-1} \gamma U$; on transforme $\hat{\beta}$ par cette transformation; on définit de même $\mathcal{C}$ et la condition (LG) donne:

$$
\tilde{\hat{\beta}}_{1}^{3}+2 \mathcal{C}_{1}^{3} \neq 0
$$

$(\mathrm{LG})_{5}$

1) $\operatorname{Si} \beta^{2}=0, \beta \neq 0, \beta \sim \tilde{\beta}=\left(\begin{array}{ll}0 & 1 \\ 0 & 0\end{array}\right), \tilde{\gamma}_{1}^{3}=0, C_{3}=0 ; C_{4}=0$;

$$
\begin{aligned}
C_{5} \equiv & -\left(\tilde{\gamma}_{3}^{1}\right)^{2}\left(\tilde{\gamma}_{1}^{1}+\tilde{\gamma}_{3}^{3}\right)-\tilde{\gamma}_{3}^{1} \tilde{\gamma}_{3}^{3}{\tilde{\beta^{\prime \prime}}}_{1}{ }_{1}^{1}+\tilde{\gamma}_{3}^{3} \tilde{\beta}^{\prime \prime}{ }_{3}^{1} \tilde{\beta}^{\prime \prime}{ }_{1}^{3}+\tilde{\beta}^{\prime \prime}{ }_{1}^{1} \tilde{\gamma}_{3}^{1} \tilde{\gamma}_{1}^{1}+\left(\tilde{\gamma}_{3}^{3}\right)^{2} \tilde{\beta}_{3}^{\prime \prime}{ }_{3}^{1} \\
& +\left(\tilde{\gamma}_{3}^{3} \tilde{\beta}_{11}^{1}-\tilde{\gamma}_{1}^{\prime 3} \tilde{\gamma}_{1}^{\prime \prime}+\tilde{\beta}_{13}^{3} \tilde{\gamma}_{1}^{1}-\tilde{\gamma}^{3} \tilde{\gamma}_{11}^{\prime \prime}\right)|\eta|+\left(\tilde{\gamma}_{3}^{3}-\tilde{\gamma}_{1}^{1}\right) D_{0} \tilde{\gamma}_{3}^{1}+\tilde{\gamma}_{3}^{3} D_{0} \tilde{\beta}^{\prime \prime}{ }_{1}^{1} \\
& +\tilde{\gamma}^{3} D_{0} \tilde{\gamma}_{3}^{\prime \prime}\left(2 \tilde{\gamma}_{3}^{1}+\tilde{\beta}^{\prime \prime}{ }_{1}^{1}\right)-2 \tilde{\gamma}_{1}^{1} D_{0} \tilde{\gamma}^{\prime 1} \tilde{\gamma}_{3}^{\prime \prime}-D_{0}^{2}{\tilde{\gamma}^{\prime}}^{1} \tilde{\gamma}_{1}^{\prime \prime}-\tilde{\gamma}^{3} D_{0}^{2} \tilde{\gamma}_{3}^{\prime \prime}+\tilde{\beta}^{\prime \prime}{ }_{1}^{1} D_{0} \tilde{\gamma}^{1} \tilde{\gamma}_{1}^{\prime \prime} \\
& +\left(-2 \tilde{\gamma}_{3}^{1} \tilde{\gamma}_{3}^{3}-\tilde{\beta}^{\prime \prime}{ }_{1}^{1} \tilde{\gamma}_{3}^{3}+2 \tilde{\gamma}^{\prime 3} D_{0} \tilde{\gamma}_{3}^{\prime \prime}\right) \mathcal{C}_{1}^{1}+\tilde{\beta}^{\prime \prime}{ }_{3}^{1} \tilde{\gamma}_{3}^{3} \mathcal{C}_{1}^{3}+\tilde{\gamma}_{3}^{3} D_{0} \mathcal{C}_{1}^{1}-\tilde{\gamma}_{3}^{3}\left(\mathcal{C}_{1}^{1}\right)^{2} \\
& +\left(-\tilde{\gamma}^{3} \cdot \partial_{\xi^{\prime}} \tilde{\gamma}_{3}^{\prime \prime} \cdot \mathcal{C}_{x 1}^{3}+\mathcal{C}_{\xi 1}^{3} \cdot D_{x^{\prime}} \tilde{\gamma}^{\prime 1} \tilde{\gamma}^{\prime \prime}{ }_{1}+\tilde{\gamma}_{3}^{3} \mathcal{C}_{\xi 1}^{1} \cdot \mathcal{C}_{x 1}^{3}\right) \neq 0
\end{aligned}
$$


2) $\operatorname{Si} \beta=0, \gamma \sim \tilde{\gamma}=\left(\begin{array}{ll}0 & 1 \\ 0 & 0\end{array}\right), \tilde{\hat{\beta}}_{1}^{3}+2 \mathcal{C}_{1}^{3}=0$

$$
C_{5}^{\prime} \equiv \tilde{\beta}_{3}^{\prime \prime}{ }_{3}^{3}{\tilde{\beta^{\prime}}}_{1}^{3}-\tilde{\beta}_{11}^{3}|\eta|+D_{0}{\tilde{\beta^{\prime}}}_{1}^{\prime 3}+{\tilde{\beta^{\prime \prime}}}_{1}{ }_{1}^{3} \mathcal{C}_{1}^{1}+D_{0} \mathcal{C}_{1}^{3}+\mathcal{C}_{1}^{3} \mathcal{C}_{1}^{1} \neq 0
$$

L: il y a 3 cas:

1) $\beta \neq 0, \beta \sim\left(\begin{array}{ll}0 & 1 \\ 0 & 0\end{array}\right): \tilde{\gamma}_{1}^{3}=0, C_{3}=C_{4}=C_{5}=0$.

2) $\beta=0, \gamma \neq 0, \gamma \sim\left(\begin{array}{ll}0 & 1 \\ 0 & 0\end{array}\right): \tilde{\hat{\beta}}_{1}^{3}+2 \mathcal{C}_{1}^{3}=0, C_{5}^{\prime}=0$.

3) $\beta=\gamma=0$, c'est-à-dire $\beta=0$ et $b_{05}^{C+1} b_{0 D}^{5}=0$, c'est-à-dire:

soit $b_{05}^{4} \neq 0$ et $b_{01}^{5}=b_{03}^{5}=0$; soit $b_{05}^{4}=0, b_{05}^{2} \neq 0, b_{01}^{5}=b_{03}^{5}=0$; soit $b_{05}^{4}=b_{05}^{2}=0$.

\subsection{Cas (LG) $)_{2}$.}

1a) $\operatorname{dét} \beta \neq 0,(\operatorname{Tr} \beta)^{2}-4$ dét $\beta \neq 0$. $\quad N$ est formé des points $0,(2,1),(4,2)$ et $(5,2)$. Les deux valeurs propres $\lambda_{i}, 1 \leq i \leq 2$, sont distinctes et non nulles. On cherche $f$ sous la forme:

$$
\begin{aligned}
f & =\sum_{1 \leq t_{1} \leq 2} f_{1}^{t_{1}}+\sum_{1 \leq t_{2} \leq 2} f_{2}^{t_{2}}+f_{3} ; \quad \text { pour } 1 \leq i \leq 2,1 \leq t_{i} \leq 2: \\
f_{i}^{t_{i}} & =e^{\psi_{i}^{t_{i}}}\left[\left(Y_{i}^{t_{i}}\right)_{0}+\cdots+\left(Y_{i}^{t_{i}}\right)_{k}+\cdots\right]
\end{aligned}
$$

$\left(D_{0} \psi_{i}^{t_{i}}\right)^{2}=\lambda_{i}|\eta| ; D_{0} \psi_{i}^{t_{i}}$ est d'ordre $1 / 2 ;\left(Y_{i}^{t_{i}}\right)_{k}$ est d'ordre: $\frac{1-k}{2}$;

$$
f_{3}=Z_{0}+\cdots+Z_{k}+\cdots ;
$$

$Z_{k}$ est d'ordre: $-\frac{k}{2}$.

Les systèmes différentiels en $Y_{1}^{t_{1}}$ et un $Y_{2}^{t_{2}}$ sont évidemment analogues; nous noterons simplement, dans le calcul suivant, $Y$ les coefficients et $\psi$ la phase.

On obtient d'abord:

(0) $Y_{0}^{C+1}=0, \quad C \in\{1,3\} ; \quad$ (1) $|\eta| Y_{1}^{C+1}+D_{0} \psi Y_{0}^{C}=0, \quad Y_{0}^{5}=0$;

(2) $|\eta| Y_{2}^{C+1}+D_{0} \psi Y_{1}^{C}+D_{0} Y_{0}^{C}-\frac{D_{*} \psi D_{0} \psi}{|\eta|} Y_{0}^{C}+\left(\beta^{\prime} Y_{0}\right)^{C}=0$;

on a noté simplement

$$
\left(\beta^{\prime} Y_{0}\right)^{C}=\sum_{1 \leq D \leq 3} \beta_{D}^{\prime C} Y_{0}^{D}
$$

et on utilisera la convention de sommation qui consiste à ne pas noter les $\sum$, lorsque il n'y a aucune ambiguité

$$
\begin{aligned}
& D_{0} \psi Y_{1}^{5}+b_{0 C}^{5} Y_{0}^{C}=0 \\
& D_{0} \psi Y_{1}^{C+1}+\left(\beta Y_{0}\right)^{C}=0
\end{aligned}
$$


d'où:

$$
\left(\beta-\frac{\left(D_{0} \psi\right)^{2}}{|\eta|} I\right) \mathcal{Y}_{0}=0 ; \quad \mathcal{Y}_{0}=\left(\begin{array}{c}
Y_{0}^{1} \\
Y_{0}^{3}
\end{array}\right)
$$

on pose: $\mathcal{Y}_{0}=U \tilde{\mathcal{Y}}_{0} ;$ de même on posera: $\mathcal{Y}_{k}=U \tilde{\mathcal{Y}}_{k} ; \mathcal{Y}_{k}=\left(\begin{array}{c}Y_{k}^{1} \\ Y_{k}^{3}\end{array}\right)$.

$$
\left(\begin{array}{cc}
\lambda_{1}-\frac{\left(D_{0} \psi\right)^{2}}{|\eta|} & 0 \\
0 & \lambda_{2}-\frac{\left(D_{0} \psi\right)^{2}}{|\eta|}
\end{array}\right) \tilde{\mathcal{Y}}_{0}=0
$$

soit si on choisit $\left(D_{0} \psi\right)^{2}=\lambda_{1}|\eta|, \lambda_{1} \neq \lambda_{2}: \tilde{\mathcal{Y}}_{0}^{3}=0$.

On pose: $\mathcal{Y}_{1}^{C+1}=\sum_{D} U_{D}^{C} \tilde{\mathcal{Y}}_{1}^{D+1}$ et de même pour les $Y_{k}^{C+1}$ : on a maintenant:

$$
\begin{aligned}
& |\eta| \tilde{\mathcal{Y}}_{1}^{C+1}+D_{0} \psi \tilde{\mathcal{Y}}_{0}^{C}=0 \\
& |\eta| \tilde{\mathcal{Y}}_{2}^{C+1}+D_{0} \psi \tilde{\mathcal{Y}}_{1}^{C}+D_{0} \tilde{\mathcal{Y}}_{1}^{C}+\left[\left(\mathcal{C}+\tilde{\beta}^{\prime}-\frac{D_{*} \psi D_{0} \psi}{|\eta|}\right) \tilde{\mathcal{Y}}_{0}\right]^{C}=0 .
\end{aligned}
$$

On note:

$$
\tilde{\gamma}_{C}^{\prime \prime}=\tilde{b}_{0 C}^{5}=\sum_{D} b_{0 D}^{5} U_{C}^{D} ; \quad \tilde{\gamma}^{\prime C}=\tilde{b}_{05}^{C+1}=\sum_{D}\left(U^{-1}\right)_{D}^{C} b_{05}^{D+1}
$$

on a:

$$
\begin{aligned}
& D_{0} \psi Y_{1}^{5}+\tilde{b}_{C}^{5} \tilde{\mathcal{Y}}_{0}^{C}=0 \\
& D_{0} \psi Y_{2}^{C+1}+D_{0} Y_{1}^{C+1}+\left(\beta Y_{1}\right)^{C}+\left(\beta^{\prime \prime} Y_{1}\right)^{C+1} \\
& \quad+b_{05}^{C+1} Y_{1}^{5}+\left[(D \beta)_{*}(\psi) Y_{0}\right]^{C}=0 \\
& {\left[\left(\beta-\frac{\left(D_{0} \psi\right)^{2}}{|\eta|} I\right) \mathcal{Y}_{1}\right]^{C}-\frac{2 D_{0} \psi}{|\eta|} D_{0} \mathcal{Y}_{0}^{C}} \\
& \quad+\left[\frac{D_{0} \psi}{|\eta|}\left(\frac{D_{*} \psi D_{0} \psi}{|\eta|}-\hat{\beta}\right)-\frac{D_{0}^{2} \psi}{|\eta|}-\frac{\gamma}{D_{0} \psi}-(D \beta)_{*}(\psi)\right] \mathcal{Y}_{0}^{C}=0
\end{aligned}
$$

Soit en multipliant par $U^{-1}$ :

$$
\begin{gathered}
\left(\tilde{\beta}-\frac{\left(D_{0} \psi\right)^{2}}{|\eta|} I\right) \tilde{\mathcal{Y}}_{1}-\frac{2 D_{0} \psi}{|\eta|} D_{0} \tilde{\mathcal{Y}}_{0} \\
+\left[\frac{\left(D_{0} \psi\right)^{2} D_{*} \psi}{|\eta|^{2}}-\frac{\tilde{\hat{\beta}} D_{0} \psi}{|\eta|}-\frac{D_{0}^{2} \psi}{|\eta|}-\frac{2 D_{0} \psi \mathcal{C}}{|\eta|}-\frac{\tilde{\gamma}}{D_{0} \psi}-\tilde{\beta} \mathcal{C}_{\xi}\left(D_{x} \psi\right)\right. \\
\left.+(D \beta)_{*}(\psi)\right] \tilde{\mathcal{Y}}_{0}=0
\end{gathered}
$$


on a remarqué que:

$$
\widetilde{D_{0} \mathcal{Y}_{0}}=D_{0} \tilde{\mathcal{Y}}_{0}+\mathcal{C} \tilde{\mathcal{Y}}_{0}
$$

La ligne $C=1$ donne:

$$
\frac{2 D_{0} \psi}{|\eta|} D_{0} \tilde{\mathcal{Y}}_{0}^{1}+W \tilde{\mathcal{Y}}_{0}^{1}=0
$$

Il reste à déterminer $\left.\tilde{\mathcal{Y}}_{0}^{1}\right|_{x_{0}=\underline{x}_{0}}$ c'est-à-dire plus précisément les $\left(\tilde{\mathcal{Y}}_{1}^{t_{1}}\right)_{0}^{1} \mid$. Le système en $Y_{2}^{t_{2}}$ est analogue; il reste à déterminer les $\left(\tilde{\mathcal{Y}}_{2}^{t_{2}}\right)_{0}^{3} \mid$. Le système en $Z$ donne, en posant $\mathcal{Z}_{0}=\left(\begin{array}{c}Z_{0}^{1} \\ Z_{0}^{3}\end{array}\right)$.

$$
\begin{gathered}
\text { (0) } Z_{0}^{C+1}=0, \quad(1) \quad Z_{1}^{C+1}=0, \quad(2) \quad|\eta| Z_{2}^{C+1}+D_{0} Z_{0}^{C}+\left(\beta^{\prime} Z_{0}\right)^{C}=0 \\
\left(\beta Z_{0}\right)^{C}+b_{5}^{C+1} Z_{0}^{5}=0 ; \quad D_{0} Z_{0}^{5}+b_{0 C}^{5} Z_{0}^{C}=0
\end{gathered}
$$

de même que précédemment

$$
\left(\tilde{\beta} \tilde{\mathcal{Z}}_{0}\right)^{C}+\tilde{b}_{5}^{C+1} Z_{0}^{5}=0
$$

d'où:

$$
\lambda_{1} \tilde{Z}_{0}^{1}+\tilde{b}_{5}^{2} Z_{0}^{5}=0 \quad \lambda_{2} \tilde{Z}_{0}^{3}+\tilde{b}_{5}^{4} Z_{0}^{5}=0
$$

et:

$$
D_{0} Z_{0}^{5}-\left(\frac{\tilde{b}_{5}^{2} \tilde{b}_{1}^{5}}{\lambda_{1}}+\frac{\tilde{b}_{5}^{4} \tilde{b}_{3}^{5}}{\lambda_{2}}\right) Z_{0}^{5}=0
$$

il suffit de déterminer $\left.Z_{0}^{5}\right|_{x_{0}=\underline{x}_{0}}$.

On impose les conditions:

$$
\sum_{t_{1}}\left(Y_{1}^{t_{1}}\right)_{0}\left|+\sum_{t_{2}}\left(Y_{2}^{t_{2}}\right)_{0}\right|=0 ; \quad \sum_{t_{1}}\left(Y_{1}^{t}\right)_{1}\left|+\sum_{t_{2}}\left(Y_{2}^{t_{2}}\right)_{1}\right|+Z_{0} \mid=I ; \quad \ldots ;
$$

on en déduit, en considérant les lignes $C$ de la 1ère équation:

$$
\sum_{t_{1}}\left(Y_{1}^{t_{1}}\right)_{0}^{C}\left|+\sum_{t_{2}}\left(Y_{2}^{t_{2}}\right)_{0}^{C}\right|=0, \quad \text { ou: } \quad \sum_{t_{1}}\left(\tilde{\mathcal{Y}}_{1}^{t_{1}}\right)_{0}^{C}\left|+\sum_{t_{2}^{\prime}}\left(\tilde{\mathcal{Y}}_{2}^{t_{2}}\right)_{0}^{C}\right|=0 ;
$$

d'où:

$$
\sum_{t_{1}}\left(\tilde{\mathcal{Y}}_{1}^{t_{1}}\right)_{0}^{1}\left|=0, \quad \sum_{t_{2}^{\prime}}\left(\tilde{\mathcal{Y}}_{2}^{t_{2}}\right)_{0}^{3}\right|=0
$$


dans la $2^{\text {ème }}$ équation, les lignes $C+1$ donnent:

$$
\sum_{t_{1}} D_{0} \psi_{1}^{t_{1}}\left(\tilde{\mathcal{Y}}_{1}^{t_{1}}\right)_{0}^{C}\left|+\sum_{t_{2}} D_{0} \psi_{2}^{t_{2}}\left(\tilde{\mathcal{Y}}_{2}^{t_{2}}\right)_{0}^{C}\right|=-|\eta| I^{C+1}
$$

d'où:

$$
\begin{aligned}
& \sum_{t_{1}} D_{0} \psi_{1}^{t_{1}}\left(\tilde{\mathcal{Y}}_{1}^{t_{1}}\right)_{0}^{C}\left|+\sum_{t_{2}} D_{0} \psi_{2}^{t_{2}}\left(\tilde{\mathcal{Y}}_{2}^{t_{2}}\right)_{0}^{C}\right|=-|\eta| \sum_{D}\left(U^{-1}\right)_{D}^{C} I^{D+1} \\
& \sum_{t_{1}} D_{0} \psi_{1}^{t_{1}}\left(\tilde{\mathcal{Y}}_{1}^{t_{1}}\right)_{0}^{1}|=-| \eta\left|\left(U^{-1}\right)_{1}^{1}\right| I^{2}+\left(U^{-1}\right)_{3}^{1} \mid I^{4} \\
& \sum_{t_{1}} D_{0} \psi_{2}^{t_{1}}\left(\tilde{\mathcal{Y}}_{2}^{t_{2}}\right)_{0}^{3}|=-| \eta\left|\left(U^{-1}\right)_{1}^{3}\right| I^{2}+\left(U^{-1}\right)_{3}^{3} \mid I^{4}
\end{aligned}
$$

on en déduit les: $\left(\tilde{\mathcal{Y}}_{1}^{t_{1}}\right)_{0}^{1} \mid$ et $\left(\tilde{\mathcal{Y}}_{2}^{t_{2}}\right)_{0}^{3} \mid$ et tous les $Y$; on a aussi à la ligne 5$) Z_{0}^{5} \mid$ qui est déterminé en fonction de $I^{5}$ et des données $\tilde{\mathcal{Y}}$ précédemment déterminées, on a ainsi tous les $Z$.

1b) $\operatorname{dét} \beta \neq 0$ et $(\operatorname{Tr} \beta)^{2}-4$ dét $\beta=0$. Les 2 valeurs propres de $\beta$ sont égales et non nulles. On suppose, en fait que $\tilde{\beta}=\left(\begin{array}{cc}\lambda & 1 \\ 0 & \lambda\end{array}\right), \lambda \neq 0$ et que:

$$
C_{3}^{\prime} \equiv \lambda\left[\tilde{\hat{\beta}}_{1}^{3}+2 \mathcal{C}_{1}^{3}+\sqrt{\lambda}|\eta| \mathcal{C}_{\xi 1}^{3} \int_{\underline{x}_{0}}^{x_{0}} D_{x} \sqrt{\lambda\left(u, x^{\prime}, \eta^{\prime}\right)} d u\right]+\tilde{\gamma}_{1}^{3}=0
$$

où $\tilde{\gamma}=U^{-1} \gamma U$; cette condition exprime l'annulation d'une fonction analytique

$$
\begin{aligned}
f & =\sum_{1 \leq t \leq 2} f_{1}^{t}+f_{2} \\
f_{1}^{t} & =e^{\psi^{t}}\left[Y_{0}^{t}+\cdots+Y_{k}^{t}+\cdots\right]
\end{aligned}
$$

$\left(D_{0} \psi^{t}\right)^{2}=\lambda|\eta| ; \psi^{t}$ est d'ordre $1 / 2 ; Y_{k}^{t}$ est d'ordre $\frac{2-k}{2} ;$

$$
f_{2}=Z_{0}+\cdots+Z_{k}+\cdots
$$

$Z_{k}$ est d'ordre $-k / 2$.

Pour déterminer les $Y$ on reprend le calcul précédent; à l'étape (2), on a:

$$
\tilde{\hat{\mathcal{Y}}}_{0}^{3}=0
$$

à l'étape (3), pour la ligne $C=3$

$$
\left[\frac{\partial_{0} \psi}{|\eta|}\left(\tilde{\hat{\beta}}_{1}^{3}+2 \mathcal{C}_{1}^{3}\right)+\frac{\tilde{\gamma}_{1}^{3}}{D_{0} \psi}+\lambda \mathcal{C}_{\xi 1}^{3}\left(D_{x^{\prime}} \psi\right)\right] \tilde{\mathcal{Y}}_{0}^{1}=0
$$


soit:

$$
\left[\lambda\left(\tilde{\hat{\beta}}_{1}^{3}+2 \mathcal{C}_{1}^{3}+D_{0} \psi \mathcal{C}_{\xi 1}^{3} D_{x^{\prime}} \psi\right)+\tilde{\gamma}_{1}^{3}\right] \tilde{\mathcal{Y}}_{0}^{1}=0
$$

qui est réalisée sans condition sur $\tilde{\mathcal{Y}}_{0}^{1}$.

Pour la ligne $C=1$, on a:

$$
\tilde{\mathcal{Y}}_{1}^{3}-\frac{2 D_{0} \psi}{|\eta|} D_{0} \tilde{\mathcal{Y}}_{0}^{1}+W \tilde{\mathcal{Y}}_{0}^{1}=0 .
$$

A l'étape (4), la ligne $C=3$ donne

$$
-\frac{2 D_{0} \psi}{|\eta|} D_{0} \tilde{\mathcal{Y}}_{1}^{3}+W^{\prime} \tilde{\mathcal{Y}}_{1}^{3}+W^{\prime \prime} D_{0} \tilde{\mathcal{Y}}_{0}^{1}+W^{\prime \prime} \tilde{\mathcal{Y}}_{0}^{1}=0
$$

d'où:

$$
D_{0}^{2} \tilde{\mathcal{Y}}_{0}^{1}+W^{I V} D_{0} \tilde{\mathcal{Y}}_{0}^{1}+W^{V} \tilde{\mathcal{Y}}_{0}^{1}=0
$$

Pour les $Z$ on reprend le calcul précédent et on obtient:

$$
\begin{aligned}
& \lambda \tilde{\mathcal{Z}}_{0}^{1}+\tilde{\mathcal{Z}}_{0}^{3}+\tilde{b}_{5}^{2} Z_{0}^{5}=0 \\
& \lambda \tilde{\mathcal{Z}}_{0}^{3}+\tilde{b}_{5}^{4} Z_{0}^{5}=0 \\
& D_{0} Z_{0}^{5}+\tilde{b}_{C}^{5} \tilde{\mathcal{Z}}_{0}^{C}=0
\end{aligned}
$$

on exprime $\tilde{\mathcal{Z}}_{0}^{3}$ et $\tilde{\mathcal{Z}}_{0}^{1}$ en fonction de $Z_{0}^{5}$ et on obtient:

$$
D_{0} Z_{0}^{5}+W Z_{0}^{5}=0
$$

On impose les conditions initiales:

$$
\sum_{t} Y_{0}^{t}\left|=0 ; \quad \sum_{t} Y_{1}^{t}\right|=0 ; \quad \sum_{t} Y_{2}^{t}\left|+Z_{0}\right|=I ; \quad \ldots ;
$$

on en déduit:

$$
\begin{aligned}
& \tilde{\mathcal{Y}}_{0}^{1}\left|=0 ; \quad \sum_{t} D_{0} \psi^{t} D_{0} \tilde{\mathcal{Y}}_{0}^{1}\right|=0 \\
& \sum_{t} D_{0} \tilde{\mathcal{Y}}_{0}^{1} \mid=-\frac{|\eta|}{2 \lambda}\left(U^{-1}\right)_{D}^{3} I^{D+1}=-\frac{\eta}{2 \lambda}\left[\left(U^{-1}\right)_{1}^{3} I^{2}+\left(U^{-1}\right)_{3}^{3} I^{4}\right]
\end{aligned}
$$

d'où: $\tilde{\mathcal{Y}}_{0}^{1}, Y_{0}^{1}$ et tous les $Y$; on a aussi, $Z_{0}^{5} \mid$ et tous les $Z$. 
1c) $\operatorname{dét} \beta \neq 0$ et $(\operatorname{Tr} \beta)^{2}-4 \operatorname{dét} \beta=0 . \quad \tilde{\beta} \sim\left(\begin{array}{ll}\lambda & 0 \\ 0 & \lambda\end{array}\right), \lambda \neq 0$.

$$
\begin{aligned}
f & =\sum_{1 \leq t \leq 2} f_{1}^{t}+f_{2} \\
f_{1}^{t} & =e^{\psi^{t}}\left[Y_{0}^{t}+\cdots+Y_{k}^{t}+\cdots\right]
\end{aligned}
$$

$\left(D_{0} \psi^{t}\right)^{2}=\lambda|\eta| ; \psi^{t}$ est d'ordre $1 / 2 ; Y_{k}$ est d'ordre $\frac{1-k}{2} ;$

$$
f_{2}=Z_{0}+\cdots+Z_{k}+\cdots
$$

$Z_{k}$ est d'ordre $-k / 2$.

On obtient:

$$
2 D_{0} \psi D_{0} \tilde{\mathcal{Y}}_{0}+W \tilde{\mathcal{Y}}_{0}=0
$$

il reste à déterminer les $\tilde{\mathcal{Y}}_{0} \mid$; on a encore:

$$
D_{0} Z_{0}^{5}+W Z_{0}^{5}=0
$$

et $Z_{0}^{5} \mid$ reste à déterminer.

On impose:

$$
\sum_{t} Y_{0}^{t}\left|=0 ; \quad \sum_{t} Y_{1}^{t}\right|+Z_{0} \mid=I ; \quad \cdots
$$

et on déterminer tous les coefficients.

1d) $\operatorname{dét} \beta \neq 0,(\operatorname{Tr} \beta)^{2}-4$ dét $\beta=0$ et $\tilde{\beta} \sim\left(\begin{array}{cc}\lambda & 1 \\ 0 & \lambda\end{array}\right), \lambda \neq 0, C_{3}^{\prime} \neq 0$.

$$
\begin{aligned}
f & =\sum_{\substack{1 \leq t \leq 2 \\
1 \leq t^{\prime} \leq 2}} f_{1}^{t t^{\prime}}+f_{2} \\
f_{1}^{t t^{\prime}} & =e^{\psi_{1}^{t}+\psi_{1}^{t t^{\prime}}}\left[Y_{0}^{t t^{\prime}}+\cdots+Y_{k}^{t t^{\prime}}+\cdots\right]
\end{aligned}
$$

$\left(D_{0} \psi_{1}^{t}\right)^{2}=\lambda|\eta| ; \psi_{1}^{t}$ est d'ordre $1 / 2 ;\left(D_{0} \psi_{2}^{t t^{\prime}}\right)^{2}=\frac{-C_{3}^{\prime}}{4 \lambda^{2}}\left(D_{0} \psi_{1}^{t}\right) ; \psi_{2}^{t t^{\prime}}$ est d'ordre $1 / 4$ $Y_{k}^{t t^{\prime}}$ est d'ordre $\frac{3-k}{4}$;

$$
f_{2}=Z_{0}+\cdots+Z_{k}+\cdots
$$

$Z_{k}$ est d'ordre $-\frac{k}{4}$.

Les systèmes en $Y$ et $Z$ sont ceux du $\S 1.51$ c). 
On obtient:

(0) (1) $Y_{0}^{C+1}=Y_{1}^{C+1}=0 ; \quad$ (2) $|\eta| Y_{2}^{C+1}+D_{0} \psi_{1} Y_{0}^{C}=0, \quad Y_{0}^{5}=0$;

(3) $|\eta| Y_{3}^{C+1}+D_{0} \psi_{1} Y_{1}^{C}+D_{0} \psi_{2} Y_{0}^{C}=0, \quad Y_{1}^{5}=0$;

(4) $\tilde{\mathcal{Y}}_{0}^{3}=0$

$$
\begin{aligned}
& |\eta| Y_{4}^{C+1}+D_{0} \psi_{1} Y_{2}^{C}+D_{0} \psi_{2} Y_{1}^{C}+D_{0} Y_{0}^{C}+\left[-\frac{D_{*} \psi_{1} D_{0} \psi_{1}}{|\eta|}+\beta^{\prime}\right] Y_{0}^{C}=0 \\
& D_{0} \psi_{1} Y_{2}^{5}+b_{05}^{5} Y_{0}^{C}=0 \quad \text { (5) } \tilde{\mathcal{Y}}_{1}^{3}-\frac{2 D_{0} \psi_{1} D_{0} \psi_{2}}{|\eta|} \tilde{\mathcal{Y}}_{0}^{1}=0 ; \quad \ldots ;
\end{aligned}
$$

(6) On considère les lignes $C+1$; après la transformation par $U$, on obtient à la $3^{\text {ème }}$ ligne:

$$
\left[4 \lambda^{2}\left(D_{0} \psi_{2}\right)^{2}+C_{3}^{\prime} D_{0} \psi_{1}\right] \tilde{\mathcal{Y}}_{0}^{1}=0
$$

qui laisse $\tilde{\mathcal{Y}}_{0}^{1}$ libre; on a aussi:

$$
\tilde{\mathcal{Y}}_{2}^{3}+\cdots-\frac{2 D_{0} \psi_{1}}{|\eta|} D_{0} \tilde{\mathcal{Y}}_{0}^{1}+W \tilde{\mathcal{Y}}_{0}^{1}=0:
$$

(7) On considère les lignes $C+1$; en fait la transformation $U$ et on obtient, en utilisant les formules précédentes:

$$
-\frac{8\left(D_{0} \psi_{1}\right)^{2} D_{0} \psi_{2}}{|\eta|} D_{0} \tilde{\mathcal{Y}}_{0}^{1}+W \tilde{\mathcal{Y}}_{0}^{1}=0
$$

il reste à déterminer la condition initiale $\tilde{\mathcal{Y}}_{0}^{1} \mid$.

Pour $Z$, on a:
(0)
(1) (2)
(3) $Z_{0}^{C+1}=\cdots=Z_{3}^{C+1}=0$;
(4) $\beta Z_{0}+b_{05}^{C+1} Z_{0}^{5}=0$,

d'où:

$$
\begin{array}{ll}
\lambda \tilde{\mathcal{Z}}_{0}^{1}+\tilde{\mathcal{Z}}_{0}^{3}+\tilde{b}_{05}^{2} Z_{0}^{5}=0, & \lambda \tilde{\mathcal{Z}}_{0}^{3}+\tilde{b}_{5}^{4} Z_{0}^{5}=0 \\
D_{0} Z_{0}^{5}+\tilde{b}_{0 C}^{5} \tilde{\mathcal{Z}}_{0}^{C}=0 ; &
\end{array}
$$

on en déduit:

$$
D_{0} Z_{0}^{5}+W Z_{0}^{5}=0
$$

il reste à déterminer $Z_{0}^{5} \mid$. 
On impose:

$\sum_{t t^{\prime}} Y_{0}^{t t^{\prime}}\left|=0 ; \quad \sum_{t t^{\prime}} Y_{1}^{t t^{\prime}}\right|=0 ; \quad \sum_{t t^{\prime}} Y_{2}^{t t^{\prime}}\left|=0 ; \quad \sum_{t t^{\prime}} Y_{3}^{t t^{\prime}}\right|+Z_{0} \mid=I ; \quad \ldots ;$

L'équation (0) implique:

$$
\sum_{t t^{\prime}} \tilde{\mathcal{Y}}_{0}^{t t^{\prime} 1} \mid=0
$$

(1) implique:

$$
\sum_{t t^{\prime}} D_{0} \psi_{1}^{t} D_{0} \psi_{2}^{t t^{\prime}} \tilde{\mathcal{Y}}_{0}^{t t^{\prime} 1} \mid=0
$$

(2) implique:

$$
\sum_{t t^{\prime}} D_{0} \psi_{1}^{t} \tilde{\mathcal{Y}}_{0}^{t t^{\prime} 1} \mid=0
$$

(3) implique:

$$
\sum_{t t^{\prime}} D_{0} \psi_{2}^{t t^{\prime}} \tilde{\mathcal{Y}}_{0}^{t t^{\prime} 1} \mid=-\frac{|\eta|}{2 \lambda}\left[\left(U^{-1}\right)_{1}^{3} I^{2}+\left(U^{-1}\right)_{3}^{3} I^{4}\right]
$$

d'où, comme précédemment, on détermine les conditions initiales et tous les $Y$; on

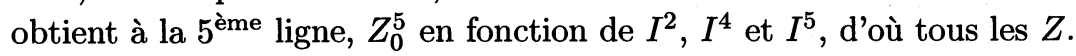

2) $\operatorname{dét} \beta=0, \operatorname{Tr} \beta \neq 0$ une valeur propre est nulle, l'autre est:

$$
\lambda=\operatorname{Tr} \beta \neq 0 ; \quad \beta \sim\left(\begin{array}{cc}
\lambda & 0 \\
0 &
\end{array}\right)=\tilde{\beta}=U^{-1} \beta U .
$$

On distingue deux cas:

2a) $\tilde{\gamma}_{3}^{3} \neq 0$.

$$
\begin{aligned}
f & =\sum_{1 \leq t \leq 2} f_{1}^{t}+\sum_{1 \leq t^{\prime} \leq 3} f_{2}^{t^{\prime}} \\
f_{1}^{t} & =e^{\psi^{t}}\left[Y_{0}^{t}+\cdots+Y_{k}^{t}+\cdots\right]
\end{aligned}
$$

$\left(D_{0} \psi^{t}\right)^{2}=\lambda|\eta| ; \psi^{t}$ est d'ordre $1 / 2 ; Y_{k}^{t}$ est d'ordre: $\frac{3-k}{6}$;

$$
f_{2}^{t^{\prime}}=e^{\psi^{t^{\prime}}}\left[Z_{0}^{t^{\prime}}+\cdots+Z_{k}^{t^{\prime}}+\cdots\right]
$$

$\left(D_{0} \chi^{t^{\prime}}\right)^{3}=-\tilde{\gamma}_{3}^{3}|\eta| ; \chi^{t_{1}}$ est d'ordre $1 / 3 ; Z_{k}^{t}$ est d'ordre: $\frac{2-k}{6}$.

Les systèmes en $Y, Z$ sont ceux du $\S 1.52 \mathrm{a}$ ).

On a, en bref:

$$
Y_{0}^{C+1}=0 ; \quad Y_{0}^{5}=0
$$




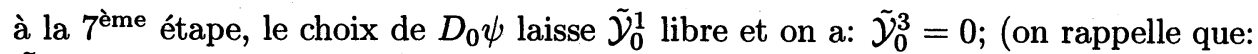
$\left.\tilde{\mathcal{Y}}_{0}^{C}=\left(U^{-1} Y_{0}\right)^{C}\right)$; à la $9^{\text {ème }}$ étape, on obtient:

$$
D_{0} \tilde{\mathcal{Y}}_{0}^{1}+W \tilde{\mathcal{Y}}_{0}^{1}=0
$$

On a:

$$
Z_{0}^{C+1}=0 ; Z_{0}^{5}=0
$$

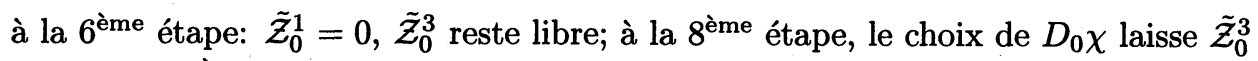
libre; à la $10^{\text {ème }}$ étape, on a:

$$
3 D_{0} \chi D_{0} \tilde{\mathcal{Z}}_{0}^{3}+W \tilde{\mathcal{Z}}_{0}^{3}=0 .
$$

Il reste donc à déterminer $\tilde{\mathcal{Y}}_{0}^{1} \mid$ et $\tilde{\mathcal{Z}}_{0}^{3} \mid$.

On impose:

$$
\sum_{t^{\prime}} Z_{0}^{t^{\prime}}\left|=0 ; \quad \sum_{t} Y_{0}^{t}\right|+\sum_{t^{\prime}} Z_{1}^{t^{\prime}}\left|=0 ; \quad \ldots ; \quad \sum_{t} Y_{3}^{t}\right|+\sum_{t^{\prime}} Z_{4}^{t^{\prime}} \mid=I ; \quad \ldots ;
$$

on obtient:

$$
\begin{aligned}
& \sum_{t^{\prime}} \tilde{\mathcal{Z}}_{0}^{t^{\prime} 3}=0 ; \quad \sum_{t} \tilde{\mathcal{Y}}_{0}^{t 1}=0 ; \quad \sum_{t^{\prime}}\left(D_{0} \chi\right)^{2} \tilde{\mathcal{Z}}_{0}^{t^{\prime} 3} \mid=0 \\
& \sum_{t} D_{0} \psi \tilde{\mathcal{Y}}_{0}^{t 1}|=-| \eta \mid \tilde{I}^{2}
\end{aligned}
$$

(en posant $\left.U^{-1} I^{C}=\tilde{I}^{C+1}\right)$

$$
\sum_{t^{\prime}} D_{0} \chi \tilde{\mathcal{Z}}_{0}^{t^{\prime} 3}|=-| \eta \mid \tilde{I}^{4}
$$

d'où les données initiales et tous les coefficients.

2b) $\tilde{\gamma}_{3}^{3}=0$.

$$
\begin{aligned}
f & =\sum_{t} f_{1}^{t}+f_{2} \\
f_{1}^{t} & =e^{\psi^{t}}\left[Y_{0}^{t}+\cdots+Y_{k}^{t}+\cdots\right] ;
\end{aligned}
$$

$\left(D_{0} \psi^{t}\right)^{2}=\lambda|\eta| ; \psi^{t}$ est d'ordre $1 / 2 ; Y_{k}^{t}$ est d'ordre $\frac{1-k}{2}$;

$$
f_{2}=Z_{0}+\cdots+Z_{k}+\cdots
$$

$Z_{k}^{t}$ est d'ordre: $\frac{2-k}{2}$.

La détermination des $Y$ et des $Z$ se fait en considérant les systèmes en $Y$ et $Z$ $\mathrm{du} 1 \mathrm{a})$ avec les nouvelles hypothèses

$$
\begin{aligned}
& Y_{0}^{C+1}=0 ; \quad \tilde{\mathcal{Y}}_{0}^{3}=0 ; \quad 2 D_{0} \psi D_{0} \tilde{\mathcal{Y}}_{0}^{1}+W \tilde{\mathcal{Y}}_{0}^{1}=0 ; \\
& Z_{0}^{C+1}=0 ; \quad \lambda \tilde{\mathcal{Z}}_{0}^{1}+\tilde{b}_{05}^{2} Z_{0}^{5}=0, \quad \tilde{b}_{05}^{4} Z_{0}^{5}=0, \quad D_{0} Z_{0}^{5}+\tilde{b}_{01}^{5} \tilde{\mathcal{Z}}_{0}^{1}+\tilde{b}_{03}^{5} \tilde{\mathcal{Z}}_{0}^{3}=0 ;
\end{aligned}
$$

on distingue: 
i) $\tilde{b}_{05}^{4} \neq 0, \tilde{b}_{03}^{5}=0$, d'où: $Z_{0}^{5}$ et $\tilde{\mathcal{Z}}_{0}^{1}=0$; à l'étape (4), on obtient:

$$
\begin{aligned}
& W D_{0} \tilde{\mathcal{Z}}_{0}^{3}+W^{\prime} \tilde{\mathcal{Z}}_{0}^{3}+\lambda \tilde{\mathcal{Z}}_{2}^{1}+\tilde{b}_{05}^{2} Z_{0}^{5}=0 \\
& -\frac{D_{0}^{2} \tilde{\mathcal{Z}}_{0}^{3}}{|\eta|}+W^{\prime \prime} D_{0} \tilde{\mathcal{Z}}_{0}^{3}+W^{\prime \prime \prime} \tilde{\mathcal{Z}}_{0}^{3}+\tilde{b}_{05}^{4} Z_{2}^{5}=0 \\
& D_{0} Z_{2}^{5}+\tilde{b}_{01}^{5} \tilde{\mathcal{Z}}_{2}^{1}+\tilde{b}_{13}^{5} \tilde{\mathcal{Z}}_{0}^{3}=0
\end{aligned}
$$

d'où en éliminant $\tilde{\mathcal{Z}}_{2}^{1}$ et $Z_{2}^{5}$ :

$$
\frac{1}{b_{05}^{4}} D_{0}^{3} \tilde{\mathcal{Z}}_{0}^{3}+W D_{0}^{2} \tilde{\mathcal{Z}}_{0}^{3}+W^{\prime} D_{0} \tilde{\mathcal{Z}}_{0}^{3}+W^{\prime \prime} \tilde{\mathcal{Z}}_{0}^{3}=0
$$

On impose les conditions initiales:

$$
Z_{0}\left|=0 ; \quad \sum_{t} Y_{0}^{t}\right|+Z_{1}=0 ; \quad \sum_{t} Y_{1}^{t}+Z_{2}=I ; \quad \ldots ;
$$

d'où:

$$
\tilde{\mathcal{Z}}_{0}^{3}\left|=0 ; \quad \sum_{t} \mathcal{Y}_{0}^{1}\right|=0 ; \quad \sum_{t} D_{0} \psi^{t} \tilde{\mathcal{Y}}_{0}^{t 1}|=-| \eta\left|\tilde{I}^{2} ; \quad D_{0} \tilde{\mathcal{Z}}_{0}^{3}\right|=-\tilde{I}^{4}
$$

$D_{0}^{2} \tilde{\mathcal{Z}}_{0}^{3} \mid$ s'exprime à l'aide de $\tilde{I}_{2}$ et $\tilde{I}^{4}$; on obtient ainsi tous les coefficients.

ii) $\tilde{b}_{05}^{4}=0$; on a:

$$
\tilde{\mathcal{Z}}_{0}^{1}=-\frac{\tilde{b}_{05}^{2}}{|\lambda|} Z_{0}^{5} ; \quad D_{0} Z_{0}^{5}-\frac{\tilde{\gamma}_{1}^{1}}{\lambda} Z_{0}^{5}+\tilde{b}_{03}^{5} \tilde{\mathcal{Z}}_{0}^{3}=0
$$

à la $4^{\text {ème }}$ étape:

$$
D_{0}^{2} \tilde{\mathcal{Z}}_{0}^{3}+W D_{0} \tilde{\mathcal{Z}}_{0}^{3}+W^{\prime} \tilde{\mathcal{Z}}_{0}^{3}+Z_{0}^{5}=0
$$

on obtient un système en $\tilde{\mathcal{Z}}_{0}^{3}$ et $Z_{0}^{5}$.

On impose les mêmes conditions initiales que dans le cas i). On obtient:

$$
\begin{gathered}
\tilde{\mathcal{Z}}_{0}^{3}\left|=0 ; \quad Z_{0}^{5}\right|=0 ; \quad \tilde{\mathcal{Y}}_{0}^{1} \mid=0 \\
\sum_{t} D_{0} \psi^{t} \tilde{\mathcal{Y}}_{0}^{t 1}|=-| \eta\left|\tilde{I}^{2} ; \quad D_{0} \tilde{\mathcal{Z}}_{0}^{3}\right|=-|\eta| \tilde{I}^{4}
\end{gathered}
$$

d'où tous les coefficients $Y$ et $Z$. 
5.2. $\operatorname{Cas}(\mathbf{L G})_{5 / 2} \cdot \beta^{2}=0 ; \beta \gamma \beta \neq 0$.

On a donc: $\beta \sim \tilde{\beta}=\left(\begin{array}{cc}0 & 1 \\ 0 & 0\end{array}\right) ; \tilde{\gamma}_{1}^{3} \neq 0$

$$
\begin{aligned}
f & =\sum_{1 \leq t \leq 5} f_{1}^{t} ; \\
f_{1}^{t} & =e^{\psi_{1}^{t}+\psi_{2}^{t}}\left[Y_{0}^{t}+\cdots+Y_{k}^{t}\right]
\end{aligned}
$$

$\left(D_{0} \psi_{1}^{t}\right)^{5}=-\tilde{\gamma}_{1}^{3}|\eta|^{2} ; \psi_{1}^{t}$ est d'ordre $\frac{2}{5}$

$$
D_{0} \psi_{2}^{t}=\frac{\left(D_{0} \psi_{1}^{t}\right)^{3}}{|\eta|} \cdot \frac{\left[\tilde{\gamma}_{1}^{1}+\tilde{\gamma}_{3}^{3}+\tilde{\hat{\beta}}_{1}^{3}+2 \mathcal{C}_{1}^{3}\right]}{5 \tilde{\gamma}_{1}^{3}}
$$

$\psi_{2}^{t}$ est d'ordre $1 / 5 ; Y_{k}^{t}$ est d'ordre: $\frac{4-k}{5}$.

On obtient le système différentiel en $Y$ du $§ 1.6$. On résume les calculs:
(0) $Y_{0}^{C+1}=0 ; \quad \ldots$;
$(3) Y_{0}^{5}=0 ; \quad \ldots ;$
(5) $\tilde{\mathcal{Y}}_{0}^{3}=0 ; \quad \ldots ;$
(7) $\left[\frac{\left(D_{0} \psi_{1}\right)^{5}}{|\eta|^{2}}+\tilde{\gamma}_{1}^{3}\right] \tilde{\mathcal{Y}}_{0}^{1}=0$,

laisse $\tilde{\mathcal{Y}}_{0}^{1}$ libre;

(8) le choix de $D_{0} \psi_{2}$ laisse $\tilde{\mathcal{Y}}_{0}^{1}$ libre;

(9) on obtient:

$$
\frac{5\left(D_{0} \psi_{1}\right)^{3}}{|\eta|^{2}} D_{0} \tilde{\mathcal{Y}}_{0}^{1}+W \tilde{\mathcal{Y}}_{0}^{1}=0
$$

On impose les conditions initiales:

$$
\sum_{t} Y_{0}^{t}\left|=\sum_{t} Y_{1}^{t}\right|=\sum_{t} Y_{2}^{t}\left|=\sum_{t} Y_{3}^{t}\right|=0 ; \quad \sum_{t} Y_{4}^{t} \mid=I ; \quad \ldots
$$

On en déduit:

$$
\begin{aligned}
& \sum_{t} \tilde{\mathcal{Y}}_{0}^{t 1}\left|=\sum_{t} D_{0} \psi^{t} \tilde{\mathcal{Y}}_{0}^{t 1}\right|=\sum_{t}\left(D_{0} \psi^{t}\right)^{2} \tilde{\mathcal{Y}}_{0}^{t 1}\left|=\sum_{t}\left(D_{0} \psi^{t}\right)^{4} \tilde{\mathcal{Y}}_{0}^{t 1}\right|=0 \\
& \left.\sum_{t}\left(D_{0} \psi^{t}\right)^{3} \tilde{\mathcal{Y}}_{0}^{t 1}|=-| \eta\right|^{2} \tilde{I}^{4}
\end{aligned}
$$

d'où les $\tilde{\mathcal{Y}}_{0}^{t 1}$ et tous les $Y$. 
5.3. Cas $(\mathbf{L G})_{3}$. On a distingué deux sous-cas

a) $\beta^{2}=0, \beta \neq 0 . \quad \beta \sim \tilde{\beta}=\left(\begin{array}{ll}0 & 1 \\ 0 & 0\end{array}\right), \tilde{\gamma}_{1}^{3}=0$,

$$
\begin{aligned}
f & =\sum_{1 \leq t \leq 3} f_{1}^{t}+f_{2} ; \\
f_{1}^{t} & =e^{\psi^{t}}\left[Y_{0}^{t}+\cdots Y_{k}^{t}+\cdots\right]
\end{aligned}
$$

$\left(D_{0} \psi^{t}\right)^{3}=-|\eta| C_{3} \neq 0 ; \psi^{t}$ est d'ordre $\frac{1}{3}, Y_{k}^{t}$ est d'ordre $\frac{3-k}{3}$;

$$
f_{2}=Z_{0}^{t}+\cdots+Z_{k}+\cdots
$$

$Z_{k}$ est d'ordre $\frac{3-k}{3}$.

On a les systèmes en $Y$ et $Z$ du $§ 1.7$.

On obtient, en résumé:

(0) $Y_{0}^{C+1}=0 ; \quad(1) \quad \ldots ; \quad$ (2) $\quad Y_{0}^{5}=0 ; \quad \ldots ; \quad(3) \quad \tilde{\mathcal{Y}}_{0}^{3}=0 ; \quad \ldots$;

(5) $\left[\left(D_{0} \psi\right)^{3}+|\eta|\left(\tilde{\gamma}_{1}^{1}+\tilde{\gamma}_{3}^{3}+\tilde{\hat{\beta}}_{1}^{3}+2 \mathcal{C}_{1}^{3}\right)\right] \tilde{\mathcal{Y}}_{0}^{1}=0 \quad$ laisse $\tilde{\mathcal{Y}}_{0}^{1}$ libre;

on obtient

(6) $3\left(D_{0} \psi\right)^{3} D_{0} \tilde{\mathcal{Y}}_{0}^{1}+W \tilde{\mathcal{Y}}_{0}^{1}=0$;

(0) $\quad Z_{0}^{C+1}=0 ; \quad \ldots ; \quad \tilde{\gamma}^{\prime 3} Z_{0}^{5}=0, \quad \tilde{\mathcal{Z}}_{0}^{3}+\tilde{\gamma}^{\prime 1} Z_{0}^{5}=0$;

$$
D_{0} Z_{0}^{5}+\tilde{\gamma}_{1}^{\prime \prime} \tilde{\mathcal{Z}}_{0}^{1}+\tilde{\gamma}_{3}^{\prime \prime} \tilde{\mathcal{Z}}_{0}^{3}=0 \text {. }
$$

On distingue deux cas

i) ${\tilde{\gamma^{\prime}}}^{3} \neq 0 . \quad{\tilde{\gamma^{\prime \prime}}}_{1}=0, Z_{0}^{5}=0, \tilde{\mathcal{Z}}_{0}^{3}=0$; à l'étape $(6)$ on obtient:

$$
D_{0}^{2} \tilde{\mathcal{Z}}_{0}^{1}+W D_{0} \tilde{\mathcal{Z}}_{0}^{1}+W \tilde{Z}_{0}^{1}=0
$$

ii) ${\tilde{\gamma^{\prime}}}^{3}=0 . \quad$ On a deux inconnues $Z_{0}^{5}$ et $\tilde{Z}_{0}^{1}$; à l'étape (6) on obtient:

$$
D_{0} \tilde{\mathcal{Z}}_{0}^{1}+W D_{0} \tilde{\mathcal{Z}}_{0}^{1}+W Z_{0}^{5}=0
$$

il reste à déterminer $\tilde{\mathcal{Z}}_{0}^{1} \mid$ et $Z_{0}^{5} \mid$.

On impose les conditions initiales:

$$
\begin{aligned}
\sum_{t} Y_{0}^{t}\left|+Z_{0}\right| & =0 ; \quad \sum_{t} Y_{1}^{t}\left|+Z_{1}\right|=0 ; \quad \sum_{t} Y_{2}^{t}\left|+Z_{2}\right|=0 \\
\sum_{t} Y_{3}^{t}\left|+Z_{3}\right| & =I ; \quad \cdots
\end{aligned}
$$

On distingue les deux sous-cas: 
i) $\quad \tilde{\gamma}^{\prime 3} \neq 0 . \quad$ On obtient:

$$
\begin{aligned}
& \sum_{t} \tilde{\mathcal{Y}}_{0}^{t 1}\left|+\tilde{\mathcal{Z}}_{0}^{1}\right|=0 \\
& \sum_{t}\left(D_{0} \psi^{t}\right)^{2} \tilde{\mathcal{Y}}_{0}^{t 1} \mid=0 \\
& \sum_{t} \tilde{\mathcal{Y}}_{0}^{t 1} \mid=\frac{|\eta| \tilde{I}^{4}}{\widetilde{\widehat{\beta}}_{1}^{3}+\tilde{\gamma}_{1}^{1}+\tilde{\gamma}_{3}^{3}+2 \mathcal{C}_{1}^{3}} ;
\end{aligned}
$$

$D_{0} \tilde{Z}_{0}^{1}$ est connu ensuite en fonction de $\tilde{I}^{2}, \tilde{I}^{5}$ et $\tilde{I}^{4}$; on obtient ainsi toutes les conditions initiales.

ii) $\quad \tilde{\gamma}^{\prime 3}=0$.

$$
\begin{array}{ll}
Z_{0}^{5} \mid=0 ; & \sum_{t} \mathcal{Y}_{0}^{t 1}\left|+\mathcal{Z}_{0}^{1}\right|=0 ; \\
\sum_{t}\left(D_{0} \psi^{t}\right)^{2} \tilde{\mathcal{Y}}_{0}^{t 1} \mid=0 ; & \sum_{t} D_{0} \psi^{t} \tilde{\mathcal{Y}}_{0}^{t 1} \mid=0
\end{array}
$$

$\sum_{t} \tilde{\mathcal{Y}}_{0}^{t 1} \mid$ est déterminé en fonction de $\tilde{I}_{4}$; on obtient toutes les conditions initiales.

b) $\beta=0, \operatorname{Tr} \gamma \neq 0$. On a donc, comme dét $\gamma=0: \gamma \sim \tilde{\gamma}=\left(\begin{array}{ll}\lambda & 0 \\ 0 & 0\end{array}\right)=U^{-1} \gamma U$, $\lambda=\operatorname{Tr} \gamma=\operatorname{Tr} \tilde{\gamma}=\tilde{\gamma}^{\prime 1} \tilde{\gamma}_{1}^{\prime \prime}$.

$$
\begin{aligned}
f & =\sum_{1 \leq t \leq 3} f_{1}^{t}+f_{2} \\
f_{1}^{t} & =e^{\psi^{t}}\left[Y_{0}^{t}+\cdots Y_{k}^{t}+\cdots\right]
\end{aligned}
$$

$\left(D_{0} \psi^{t}\right)^{3}=-\lambda|\eta| ; \psi^{t}$ est d'ordre $\frac{1}{3}, Y_{k}^{t}$ est d'ordre $\frac{2-k}{3} ;$

$$
f_{2}=Z_{0}^{t}+\cdots+Z_{k}^{t}+\cdots,
$$

$Z_{k}$ est d'ordre $\frac{3-k}{3}$.

On a

$$
\begin{aligned}
& Y_{0}^{C+1}=0 ; \quad \ldots ; \quad Y_{0}^{5}=0 ; \quad \ldots \\
& {\left[\gamma|\eta|+\left(D_{0} \psi\right)^{3} I\right] \mathcal{Y}_{0}^{C}=0}
\end{aligned}
$$

d'où:

$$
\left[\tilde{\gamma}|\eta|+\left(D_{0} \psi\right)^{3} I\right] \tilde{\mathcal{Y}}_{0}^{C}=0
$$

$\tilde{\mathcal{Y}}_{0}^{3}=0 ;$ à l'étape $(5)$

$$
3 D_{0} \psi D_{0} \tilde{\mathcal{Y}}_{0}^{1}+W \tilde{\mathcal{Y}}_{0}^{1}=0
$$


on a aussi

$$
\begin{aligned}
& \text { (0) } Z_{0}^{C+1}=0 ; \quad \ldots ; \quad \tilde{\gamma}^{\prime 1} Z_{0}^{5}=0, \text { d'où } Z_{0}^{5}=0 \\
& \tilde{\gamma}_{1}^{\prime \prime} \tilde{\mathcal{Z}}_{0}^{1}=0, \text { d'où } \tilde{\mathcal{Z}}_{0}^{1}=0 ; \quad \ldots \text {; }
\end{aligned}
$$

à l'étape (6), on a

$$
D_{0}^{2} \tilde{\mathcal{Z}}_{0}^{3}+W D_{0} \tilde{\mathcal{Z}}_{0}^{3}+W^{\prime} \tilde{Z}_{0}^{3}=0
$$

On impose les conditions initiales:

$$
Z_{0}\left|=0 ; \quad \sum_{t} Y_{0}^{t}\right|+Z_{1}\left|=0 ; \quad \sum_{t} Y_{1}^{t}\right|+Z_{2}\left|=0 ; \quad \sum_{t} Y_{2}^{t}\right|+Z_{3} \mid=I ; \quad \cdots
$$

on en déduit

$$
\begin{array}{ll}
\tilde{\mathcal{Z}}_{0}^{3}=0 ; & \sum_{t} \tilde{\mathcal{Y}}_{0}^{t 1}\left|=0 ; \quad \sum_{t}\left(D_{0} \psi^{t}\right)^{2} \tilde{\mathcal{Y}}_{0}^{t 1}\right|=0 \\
\sum_{t} D_{0} \psi^{t} \tilde{\mathcal{Y}}_{0}^{t 1}|=-| \eta \mid \tilde{I}^{2} ; & D_{0} \tilde{\mathcal{Z}}_{0}^{3}=-|\eta| \tilde{I}^{4}
\end{array}
$$

d'où tous les coefficients $Y$ et $Z$.

5.4. Cas $(\mathbf{L G})_{4}$. On distingue les deux cas:

a) $\beta^{2}=0, \beta \neq 0, \quad$ c'est-à-dire $\beta \sim \tilde{\beta}=\left(\begin{array}{ll}0 & 1 \\ 0 & 0\end{array}\right) ; \tilde{\gamma}_{1}^{3}=0 ; C_{3}=0, C_{4} \neq 0$.

$$
\begin{aligned}
f & =\sum_{1 \leq t \leq 4} f_{1}^{t}+f_{2} \\
f_{1}^{t} & =e^{\psi^{t}}\left[Y_{0}^{t}+\cdots Y_{k}^{t}+\cdots\right]
\end{aligned}
$$

$\left(D_{0} \psi^{t}\right)^{4}=C_{4}|\eta| ; \psi^{t}$ est d'ordre $\frac{1}{4}, Y_{k}^{t}$ est d'ordre $\frac{5-k}{4} ;$

$$
f_{2}=Z_{0}^{t}+\cdots+Z_{k}^{t}+\cdots
$$

$Z_{k}$ est d'ordre $\frac{4-k}{4}$.

On a les systèmes en $Y$ et $Z$ du $§ 1.8$. On a en résumé:

(0) $Y_{0}^{C+1}=0 ; \quad \ldots$;

(3) $Y_{0}^{5}=0$,

(4) $\tilde{Y}_{0}^{3}=0$,

(5) $\tilde{\mathcal{Y}}_{1}^{3}=\frac{\tilde{\gamma}_{1}^{1}}{D_{0} \psi} \tilde{\mathcal{Y}}_{0}^{1} \quad \ldots$;

(6) $\tilde{\mathcal{Y}}_{2}^{3}=\cdots-\frac{\tilde{\gamma}_{1}^{1}}{\left(D_{0} \psi\right)^{2}}+\left[\frac{\tilde{\gamma}_{3}^{1} \tilde{\gamma}_{1}^{1}}{\left(D_{0} \psi\right)^{2}}+\frac{\left(D_{0} \psi\right)^{2}}{|\eta|}-\frac{\tilde{\gamma}^{\prime 1}}{D_{0} \psi} D_{0}\left(\frac{\tilde{\gamma}_{1}^{\prime \prime}}{D_{0} \psi}\right)\right] \tilde{\mathcal{Y}}_{0}^{1}$ 
(7) $\tilde{\mathcal{Y}}_{3}^{3}$ est calculé à l'aide de $D_{0}^{2} \tilde{\mathcal{Y}}_{0}^{1}, D_{0} \tilde{\mathcal{Y}}_{0}^{1}, \tilde{\mathcal{Y}}_{0}^{1}$, et des termes de récurrence $\tilde{\mathcal{Y}}_{2}, \tilde{\mathcal{Y}}_{1}$;

(8) on obtient:

$$
\left[\left(D_{0} \psi\right)^{4}-\eta C_{4}\right] \tilde{\mathcal{Y}}_{0}^{1}=0
$$

$\tilde{\mathcal{Y}}_{0}^{1}$ reste libre; (9) on obtient:

$$
4 \frac{\left(D_{0} \psi\right)^{3}}{|\eta|} D_{0} \tilde{\mathcal{Y}}_{0}^{1}+W \tilde{\mathcal{Y}}_{0}^{1}=0
$$

il reste à déterminer $\tilde{\mathcal{Y}}_{0}^{1} \mid$.

Pour les $Z$, on a

$$
\begin{array}{ll}
\text { (0) } & (1) \quad(2) \quad(3) \quad Z_{0}^{C+1}=\cdots=Z_{3}^{C+1}=0 ; \quad \text { (4) } \beta Z_{0}+b_{05}^{C+1} Z_{0}^{5}=0 \\
& D_{0} Z_{0}^{5}+b_{0 C}^{5} Z_{0}^{C}=0 \\
& |\eta| Z_{4}^{C+1}+D_{0} Z_{0}^{C}+\left(\beta^{\prime} Z_{0}\right) C=0
\end{array}
$$

On distingue deux cas

i) $\quad \tilde{\gamma}^{\prime 3} \neq 0, \quad$ d'où $Z_{0}^{5}=0, \tilde{\gamma}_{1}^{\prime \prime}=0$ et $\tilde{\mathcal{Z}}_{0}^{3}=0$;

$$
\begin{array}{ll}
\text { (5) } Z_{1}^{5}=0, \tilde{\mathcal{Z}}_{1}^{3}=0 ; \quad(6) \quad Z_{2}^{5}=0, \tilde{\mathcal{Z}}_{2}^{3}=0 ; \quad \text { (7) } Z_{3}^{5}=0, \tilde{\mathcal{Z}}_{3}^{3}=0 ; \\
\text { (8) } \quad\left(\beta Z_{4}\right)^{C}+b_{05}^{C+1} Z_{4}^{5}-\frac{D_{0}^{2} Z_{0}^{C}}{|\eta|}-\frac{\left(\hat{\beta} D_{0} Z_{0}\right)^{C}}{|\eta|} \\
\quad+\left(-\frac{\beta^{\prime \prime} \beta^{\prime}}{|\eta|}-\frac{D_{0} \beta^{\prime}}{|\eta|}+\beta_{1}\right) Z_{0}^{C}+b_{15}^{C+1} Z_{0}^{5}+(D \beta)_{*}\left(Z_{0}\right)^{C}=0 \\
D_{0} Z_{4}^{5}+b_{0 C}^{5} Z_{4}^{C}+b_{1 C}^{5} Z_{0}^{C}=0
\end{array}
$$

On remplaçe par les termes transformés en $\sim$ la ligne $C=3$ donne $\tilde{\gamma}^{\prime 3} Z_{4}^{5}$ en fonction de $D_{0} \tilde{\mathcal{Z}}_{0}^{1}$ et $\tilde{\mathcal{Z}}_{0}^{1}$; la ligne $C=1$ donne $\tilde{\mathcal{Z}}_{4}^{3}$ en fonction de $D_{0}^{2} \tilde{\mathcal{Z}}_{0}^{1}, D_{0} \tilde{\mathcal{Z}}_{0}^{1}$ et $\tilde{\mathcal{Z}}_{0}^{1}$; on reporte la dernière équation et on obtient:

$$
C_{4} D_{0} \tilde{\mathcal{Z}}_{0}^{1}+W \tilde{\mathcal{Z}}_{0}^{1}=0
$$

il reste à déterminer $\tilde{Z}_{0}^{1} \mid$. On impose les conditions initiales:

$$
\sum_{t} Y_{0}^{t}\left|=0 ; \quad \sum_{t} Y_{1}^{t}\right|+Z_{0}^{t}\left|=0 ; \quad \ldots ; \quad \sum_{t} Y_{5}^{t}\right|+Z_{4}^{t} \mid=I ; \quad \ldots ;
$$

on obtient ainsi $\tilde{\mathcal{Z}}_{0}^{1}$ et $\tilde{\mathcal{Y}}_{0}^{1}$, puis tous les coefficients $Y$ et $Z$. 
ii) $\quad \tilde{\gamma}^{\prime 3}=0 . \quad$ On a

$$
\tilde{\mathcal{Z}}_{0}^{3}+\tilde{b}_{05}^{2} Z_{0}^{5}=0
$$

$\tilde{\mathcal{Z}}_{0}^{1}$ s'exprime aussi à l'aide de $Z_{0}^{5}$ et on obtient finalement:

$$
D_{0} Z_{0}^{5}+W Z_{0}^{5}=0
$$

On impose les conditions initiales du (i) et on obtient $\tilde{\mathcal{Y}}_{0}^{1}$ et $Z_{0}^{5}$, puis toutes les coefficients $Y$ et $Z$.

b) $\beta=0, \operatorname{Tr} \gamma=0, M_{4}^{\prime} \neq 0 . \quad$ On a: $\gamma \sim \tilde{\gamma}=\left(\begin{array}{ll}0 & 1 \\ 0 & 0\end{array}\right)=U^{-1} \gamma U$; on transforme aussi $\hat{\beta}$ par cette transformation:

$$
\begin{gathered}
\widetilde{\widehat{\beta}}_{1}^{3}+2 \mathcal{C}_{1}^{3} \neq 0 . \\
f=\sum_{1 \leq t \leq 4} f_{1}^{t}+f_{2} \\
f_{1}^{t}=e^{\psi^{t}\left[Y_{0}^{t}+\cdots Y_{k}^{t}+\cdots\right]}
\end{gathered}
$$

$\left(D_{0} \psi^{t}\right)^{4}=|\eta|\left(\widetilde{\widehat{\beta}}_{1}^{3}+2 \mathcal{C}_{1}^{3}\right) \neq 0 ; \psi^{t}$ est d'ordre $\frac{1}{4}, Y_{k}^{t}$ est d'ordre $\frac{4-k}{4} ;$

$$
f_{2}=Z_{0}^{t}+\cdots+Z_{k}+\cdots ;
$$

$Z_{k}$ est d'ordre $\frac{4-k}{4}$.

On résume les calculs en $Y$ :
(0) $Y_{0}^{C+1}=0$;
(3) $Y_{0}^{5}=0 \ldots$;
(5) $\gamma Y_{0}=0$, d'où $\tilde{\mathcal{Y}}_{0}^{3}=0$;
(6) $\tilde{\mathcal{Y}}_{1}^{3}+\frac{\left(D_{0} \psi\right)^{3}}{|\eta|} \tilde{\mathcal{Y}}_{0}^{1}=0$;
(7) $\left[\left(D_{0} \psi^{t}\right)^{4}-|\eta|\left(\widetilde{\widehat{\beta}}_{1}^{3}+2 \mathcal{C}_{1}^{3}\right)\right] \tilde{\mathcal{Y}}_{0}^{1}=0$;

$\tilde{\mathcal{Y}}_{0}^{1}$ reste libre; on obtient aussi $\tilde{\mathcal{Y}}_{3}^{2}$ en fonction de $D_{0} \tilde{\mathcal{Y}}_{0}^{1}$;

(8) $D_{0} \tilde{\mathcal{Y}}_{0}^{1}+W \tilde{\mathcal{Y}}_{0}^{1}=0$

Pour les $Z$ on a

$$
Z_{0}^{5}=0 ; \quad \tilde{\mathcal{Z}}_{0}^{3}=0 ; \quad D_{0} \tilde{\mathcal{Z}}_{0}^{1}+W \tilde{\mathcal{Z}}_{0}^{1}=0 .
$$

On impose les conditions initiales:

$$
\sum_{t} Y_{0}^{t}\left|+Z_{0}^{t}\right|=0 ; \quad \ldots ; \quad \sum_{t} Y_{4}^{t}\left|+Z_{4}^{t}\right|=I ; \quad \ldots ;
$$

et on détermine $\tilde{\mathcal{Z}}_{0}^{1}$ et $\tilde{\mathcal{Y}}_{0}^{1}$ et tous les coefficients. 
5.5. Cas $(\mathbf{L G})_{5}$. On distingue les 2 cas

a) $\beta^{2}=0, \beta \neq 0 . \quad$ c'est-à-dire $\beta \sim \tilde{\beta}=\left(\begin{array}{cc}0 & 1 \\ 0 & 0\end{array}\right) . \tilde{\gamma}_{1}^{3}=0 ; C_{3}=0 ; C_{4}=0$; $C_{5} \neq 0$.

$$
\begin{aligned}
f & =\sum_{1 \leq t \leq 5} f_{1}^{t} \\
f_{1}^{t} & =e^{\psi^{t}}\left[Y_{0}^{t}+\cdots Y_{k}^{t}+\cdots\right]
\end{aligned}
$$

$\left(D_{0} \psi^{t}\right)^{5}=C_{5}|\eta| ; \psi^{t}$ est d'ordre $\frac{1}{5}, Y_{k}^{t}$ est d'ordre $\frac{7-k}{5}$.

On a le système en $Y$ du $\S 1.9$; les calculs sont longs et nous ne le détaillons pas.

On résume les calculs en $Y$ :
(0) $Y_{0}^{C+1}=0$;
(4) $Y_{0}^{5}=0 ; \quad \ldots$;
(5) $\tilde{Y}_{0}^{3}=0 ; \quad \ldots ;$
(11) $\left[\left(D_{0} \psi^{t}\right)^{5}-C_{5}|\eta|\right] \tilde{\mathcal{Y}}_{0}^{1}=0$

$\tilde{\mathcal{Y}}_{0}^{1}$ reste libre;

(12) $5 C_{5} D_{0} \tilde{\mathcal{Y}}_{0}^{1}+W \tilde{\mathcal{Y}}_{0}^{1}=0$

On impose les conditions initiales:

$$
\sum_{t} Y_{0}^{t}\left|=\cdots=\sum_{t} Y_{0}^{t}\right|=0 ; \quad \sum_{t} Y_{7}^{t} \mid=I ; \quad \cdots
$$

On distingue les deux cas $\tilde{\gamma}^{\prime 3} \neq 0$ et $\tilde{\gamma}^{\prime 3}=0$; on obtient dans chaque cas

$$
\begin{array}{lll}
\sum_{t} \tilde{Y}_{0}^{t 1} \mid=0 ; & \sum_{t} D_{0} \psi^{t} \tilde{Y}_{0}^{t 1} \mid=0 ; \quad & \sum_{t}\left(D_{0} \psi^{t}\right)^{2} \tilde{Y}_{0}^{t 1} \mid=0 \\
\sum_{t}\left(D_{0} \psi^{t}\right)^{4} \tilde{Y}_{0}^{t 1} \mid=0 ; & \left.\sum_{t}\left(D_{0} \psi^{t}\right)^{3} \tilde{Y}_{0}^{t 1}|=| \eta\right|^{2} \tilde{I}^{4}
\end{array}
$$

On en déduit la détermination des $\tilde{\mathcal{Y}}_{0}^{t 1}$ et de tous les coefficients.

b) $\beta=0, \operatorname{Tr} \gamma=0 . \quad$ On a $\gamma \sim \tilde{\gamma}=\left(\begin{array}{ll}0 & 1 \\ 0 & 0\end{array}\right) \cdot \widetilde{\widehat{\beta}}_{1}^{3}+2 \mathcal{C}_{1}^{3}=0 ; C_{5}^{\prime} \neq 0$.

$$
\begin{aligned}
f & =\sum_{1 \leq t \leq 5} f_{1}^{t} \\
f_{1}^{t} & =e^{\psi^{t}}\left[Y_{0}^{t}+\cdots Y_{k}^{t}+\cdots\right]
\end{aligned}
$$

$\left(D_{0} \psi^{t}\right)^{5}=C_{5}^{\prime}|\eta| ; \psi^{t}$ est d'ordre $\frac{1}{5}, Y_{k}^{t}$ est d'ordre $\frac{6-k}{5}$. 
On a:
(0) $Y_{0}^{C+1}=0$;
(4) $Y_{0}^{5}=0$
(5) $\tilde{Y}_{0}^{3}=0 ; \quad \ldots$;
(10) $\quad\left[\left(D_{0} \psi^{t}\right)^{5}-C_{5}^{\prime}|\eta|\right] \tilde{\mathcal{Y}}_{0}^{1}=0$;

$\tilde{\mathcal{Y}}_{0}^{1}$ reste libre;

(11) $5 C_{5} D_{0} \tilde{\mathcal{Y}}_{0}^{1}+W \tilde{\mathcal{Y}}_{0}^{1}=0$

On impose les conditions initiales:

$$
\sum_{t} Y_{0}^{t}\left|=\cdots=\sum_{t} Y_{5}^{t}\right|=0 ; \quad \sum_{t} Y_{6}^{t} \mid=I ; \quad \cdots
$$

On obtient:

$$
\begin{gathered}
\sum_{t} \tilde{Y}_{0}^{t 1}\left|=0 ; \quad \sum_{t} D_{0} \psi^{t} \tilde{Y}_{0}^{t 1}\right|=0 ; \quad \sum_{t}\left(D_{0} \psi^{t}\right)^{2} \tilde{Y}_{0}^{t 1} \mid=0 \\
\sum_{t}\left(D_{0} \psi^{t}\right)^{3} \tilde{Y}_{0}^{t 1}\left|=0 ; \quad \sum_{t}\left(D_{0} \psi^{t}\right)^{4} \tilde{Y}_{0}^{t 1}\right|=|\eta|^{2} \tilde{I}^{4}
\end{gathered}
$$

d'où tous les coefficients.

5.6. Cas L. Toutes les conditions sont satisfaites. Le diagramme est vide.

$$
f=Y_{0}+\cdots+Y_{k}+\cdots
$$

Le système en $Y$ est le même que celui des cas L précédents.

a) $\beta \neq 0 . \quad Y_{k}$ est d'ordre $2-k$. On a $\beta \sim \tilde{\beta}=\left(\begin{array}{ll}0 & 1 \\ 0 & 0\end{array}\right)$; on obtient:

$$
\text { (0) } Y_{0}^{C+1}=0 ; \quad(1) \quad|\eta| Y_{1}^{C+1}+D_{0} Y_{0}^{C}+\beta^{\prime} Y_{0}^{C}=0 ; \quad \tilde{\gamma}^{3} Y_{0}^{5}=0 ;
$$

on est donc conduit à distinguer deux sous-cas.

i) $\quad \tilde{\gamma}^{3} \neq 0 . \quad \tilde{Y}_{0}^{5}=0, \widetilde{\widehat{\gamma}}_{1}^{\prime \prime}=0, \tilde{Y}_{0}^{3}=0 ;$ à l'étape $(2)$ on a

$$
\tilde{\mathcal{Y}}_{1}^{3}+\tilde{\gamma}^{\prime 1} Y_{1}^{5}-\frac{D_{0}^{2} \tilde{\mathcal{Y}}_{0}^{1}}{|\eta|}-\frac{\left({\widetilde{\beta^{\prime \prime}}}_{1}^{1}+2 \mathcal{C}_{1}^{1}\right)}{|\eta|} D_{0} \tilde{\mathcal{Y}}_{0}^{1}+W \tilde{\mathcal{Y}}_{0}^{1}=0
$$

(on doit, en fait, préciser les coefficients de $\tilde{\mathcal{Y}}_{0}^{1}$, nous laissons ce calcul au lecteur)

$$
\begin{gathered}
\tilde{\gamma}^{3} Y_{1}^{5}-\frac{\left(\widetilde{\widehat{\beta}}_{1}^{3}+2 \mathcal{C}_{1}^{3}\right)}{|\eta|} D_{0} \tilde{\mathcal{Y}}_{0}^{1}+W \tilde{\mathcal{Y}}_{0}^{1}=0 \\
D_{0} Y_{1}^{5}+\tilde{\gamma}_{3}^{\prime \prime} \tilde{\mathcal{Y}}_{1}^{3}+W \mathcal{Y}_{0}^{1}-\tilde{\gamma}_{3}^{\prime \prime} \mathcal{C}_{\xi 1}^{3} D_{x^{\prime}} Y_{0}^{1}=0
\end{gathered}
$$


on reporte $\tilde{\mathcal{Y}}_{1}^{3}$ et $Y_{1}^{5}$ dans la dernière équation; compte tenu des conditions $\mathrm{L}$, les coefficients de $D_{0}^{2} \tilde{\mathcal{Y}}_{0}^{1}, D_{0} \tilde{\mathcal{Y}}_{0}^{1}$ et $\tilde{\mathcal{Y}}_{0}^{1}$ sont nuls; à l'étape (3) par des calculs analogues mais compliqués, on obtient l'équation de propagation

$$
D_{0}^{5} \tilde{\mathcal{Y}}_{0}^{1}+W D_{0}^{4} \tilde{\mathcal{Y}}_{0}^{1}+W^{\prime} D_{0}^{3} \tilde{\mathcal{Y}}_{0}^{1}+W^{\prime \prime} D_{0}^{2} \tilde{\mathcal{Y}}_{0}^{1}+W^{\prime \prime \prime} D_{0} \tilde{\mathcal{Y}}_{0}^{1}+W^{I V} \tilde{\mathcal{Y}}_{0}^{1}=0
$$

On impose les conditions initiales

$$
Y_{0}\left|=0 ; \quad Y_{1}\right|=0 ; \quad Y_{2} \mid=I ; \quad \cdots ;
$$

on en déduit
(0) $\tilde{\mathcal{Y}}_{0}^{1} \mid=0$;
(1) $D_{0} \tilde{\mathcal{Y}}_{0}^{1} \mid=0$
$D_{0}^{2} \tilde{\mathcal{Y}}_{0}^{1} \mid=0$
(2) $\left.\quad D_{0}^{3} \tilde{\mathcal{Y}}_{0}^{1}|=-| \eta\right|^{2} \tilde{I}^{4}$

$D_{0}^{4} \tilde{\mathcal{Y}}_{0}^{1} \mid$ s'exprime à l'aide de $\tilde{I}^{4}$ et de $I^{5}$; on obtient ainsi $\tilde{\mathcal{Y}}_{0}^{1}$ et tous les $Y_{0}$, puis tous les coefficients.

ii) $\tilde{\gamma}^{3}=0 . \quad$ On a

$$
\text { (1) } \tilde{Y}_{0}^{3}+\tilde{\gamma}^{\prime 1} Y_{0}^{5}=0, D_{0} Y_{0}^{5}+\tilde{\gamma}^{\prime \prime}{ }_{1} Y_{0}^{1}-\tilde{\gamma}_{3}^{1} Y_{0}^{5}=0
$$

les "inconnues" sont a priori $\tilde{\mathcal{Y}}_{0}^{1}$ et $Y_{0}^{5}$; à la deuxième étape, on doit encore diviser en sous-cas:

ii) ${ }^{\prime} \tilde{\gamma}_{1}^{\prime \prime} \neq 0 . \quad$ On a $K=0$, d'après la condition $C_{5} \equiv K \tilde{\gamma}_{1}^{\prime \prime}=0 ; Y_{0}^{5}$ reste libre. À la troisième étape, on obtient, en considérant les $(C+1)$-ièmes lignes

$$
\beta \mathcal{Y}_{2}+\gamma^{\prime} Y_{2}^{5}-\frac{D_{0}^{2} \mathcal{Y}_{1}}{|\eta|}+\text { d'autres terme qu'on doit expliciter }=0
$$

on transforme par multiplication par $U^{-1}$ :

$$
\tilde{\beta} \tilde{\mathcal{Y}}_{2}-\frac{D_{0}^{2} \tilde{\mathcal{Y}}_{1}}{|\eta|}+\cdots=0
$$

dans la ligne $C=3$, le terme en $\left(\tilde{\beta} \tilde{\mathcal{Y}}_{2}\right)^{3}$ disparait et il reste:

$$
D_{0}^{4} \tilde{\mathcal{Y}}_{0}^{1}+W^{\prime} D_{0}^{3} \tilde{\mathcal{Y}}_{0}^{1}+W^{\prime \prime} D_{0}^{2} \tilde{\mathcal{Y}}_{0}^{1}+W^{\prime \prime \prime} D_{0} \tilde{\mathcal{Y}}_{0}^{1}+W^{I V} \tilde{\mathcal{Y}}_{0}^{5}=0
$$

on doit obtenir les conditions initiales:

$$
\mathcal{Y}_{0}^{1}\left|, D_{0} \tilde{\mathcal{Y}}_{0}^{1}\right|, D_{0}^{2} \tilde{\mathcal{Y}}_{0}^{1}\left|, D_{0}^{3} \tilde{\mathcal{Y}}_{0}^{1}\right|, Y_{0}^{5} \mid
$$

On impose les conditions initiales précédentes; on en déduit

$$
\mathcal{Y}_{0}^{1}\left|=0, Y_{0}^{5}\right|=0, D_{0} \mathcal{Y}_{0}^{1}\left|=0, D_{0}^{2} \tilde{\mathcal{Y}}_{0}^{1}\right|=0,\left.D_{0}^{3} \tilde{\mathcal{Y}}_{0}^{1}|=-| \eta\right|^{2} \tilde{I}^{4}
$$


ii) $\tilde{\gamma}_{1}^{\prime \prime}=0 . \quad$ On doit distinguer deux sous-cas

ii $\left._{1}^{\prime \prime}\right) K \neq 0$. On a $Y_{0}^{5}=0$. À la troisième étape, on obtient

$$
D_{0}^{4} \tilde{\mathcal{Y}}_{0}^{1}+W^{\prime} D_{0}^{3} \tilde{\mathcal{Y}}_{0}^{1}+W^{\prime \prime} D_{0}^{2} \tilde{\mathcal{Y}}_{0}^{1}+W^{\prime \prime \prime} D_{0} \tilde{\mathcal{Y}}_{0}^{1}+W^{I V} \tilde{\mathcal{Y}}_{0}^{1}=0
$$

On impose les mêmes conditions initiales. On obtient

$$
\tilde{\mathcal{Y}}_{0}^{1}\left|, D_{0} \tilde{\mathcal{Y}}_{0}^{1}\right|, D_{0}^{2} \tilde{\mathcal{Y}}_{0}^{1}\left|, D_{0}^{3} \tilde{\mathcal{Y}}_{0}^{1}\right|=-|\eta|^{2} \tilde{I}^{4}
$$

$\left.\mathrm{ii}_{2}^{\prime \prime}\right) K=0$. À la troisième étape, on obtient, comme au cas ii)' un'équation d'ordre 4 en $\tilde{\mathcal{Y}}_{0}^{1}$ et d'ordre 0 en $Y_{0}^{5}$; la détermination des conditions initiales se fait aussi comme dans le cas ii)'.

b) $\beta=0, \gamma \neq 0 . \quad Y_{k}$ est d'ordre $2-k . \gamma \sim \tilde{\gamma}=\left(\begin{array}{ll}0 & 1 \\ 0 & 0\end{array}\right)$.

On obtient $Y_{0}^{C+1}=0 ; Y_{0}^{5}=0, \tilde{\mathcal{Y}}_{0}^{3}=0$; à la troisième étape, on a

$$
D_{0}^{5} \tilde{\mathcal{Y}}_{0}^{1}+W D_{0}^{4} \tilde{\mathcal{Y}}_{0}^{1}+W^{\prime} D_{0}^{3} \tilde{\mathcal{Y}}_{0}^{1}+W^{\prime \prime} D_{0}^{2} \tilde{\mathcal{Y}}_{0}^{1}+W^{\prime \prime \prime} D_{0} \tilde{\mathcal{Y}}_{0}^{1}+W^{I V} \tilde{\mathcal{Y}}_{0}^{1}=0
$$

On impose

$$
Y_{0}\left|=0 ; \quad Y_{1}\right|=0 ; \quad Y_{2} \mid=I ; \quad \ldots ;
$$

on obtient

$$
\tilde{\mathcal{Y}}_{0}^{1}\left|=0=D_{0} \tilde{\mathcal{Y}}_{0}^{1}\right|=D_{0}^{2} \tilde{\mathcal{Y}}_{0}^{1}\left|=D_{0}^{3} \tilde{\mathcal{Y}}_{0}^{1}\right|
$$

et

$$
D_{0}^{4} \tilde{\mathcal{Y}}_{0}^{1}=|\eta|^{2} \tilde{I}^{4}
$$

c) $\beta=0, \gamma=0 . \quad Y_{k}$ est d'ordre $1-k$. On distingue deux cas.

i) $b_{05}^{4} \neq 0$. d'où $b_{01}^{5}=b_{03}^{5}=0$. On a $Y_{0}^{C+1}=0, Y_{0}^{5}=0$; on obtient:

$$
\begin{aligned}
& D_{0}^{3} Y_{0}^{3}+W D_{0}^{2} Y_{0}^{3}+W^{\prime} D_{0} Y_{0}^{3}+W^{\prime \prime} D_{0}^{2} Y_{0}^{1}+W^{\prime \prime \prime} Y_{0}^{3}+W^{I V} Y_{0}^{1}=0 \\
& b_{05}^{4} D_{0}^{2} Y_{0}^{1}-b_{05}^{2} D_{0}^{2} Y_{0}^{3}+W^{V} D_{0} Y_{0}^{3}+W^{V I} D_{0} Y_{0}^{1}+W^{V I I} Y_{0}^{3}+W^{V I I I} Y_{0}^{1}=0
\end{aligned}
$$

On impose:

$$
Y_{0}\left|=0 ; \quad Y_{1}\right|=I ; \quad \ldots ;
$$

on obtient

$$
\begin{gathered}
Y_{0}^{1}=0=Y_{0}^{3}\left|; \quad D_{0} Y_{0}^{1}\right|=-|\eta| I^{2} ; \quad D_{0} Y_{0}^{3}|=-| \eta \mid I^{4} \\
D_{0}^{2} Y_{0}^{3}=|\eta|\left(b_{05}^{4} I^{5}-\hat{\beta}_{1}^{3} I^{2}-\hat{\beta}_{3}^{3} I^{4}\right),
\end{gathered}
$$

d'où les coefficients. 
ii) $b_{05}^{4}=0 . \quad$ On distingue

ii) ${ }^{\prime} b_{05}^{2} \neq 0, b_{01}^{5}=b_{03}^{5}=0 . \quad$ On a $Y_{0}^{C+1}=0, Y_{0}^{5}=0$;

$$
\begin{aligned}
& b_{05}^{2} Y_{1}^{5}-\frac{D_{0}^{2} Y_{0}^{1}}{|\eta|}+W D_{0} Y_{0}^{1}+W^{\prime} D_{0} Y_{0}^{3}+W^{\prime \prime} Y_{0}^{1}+W^{\prime \prime \prime} Y_{0}^{3}=0 \\
& -\frac{D_{0}^{2} Y_{0}^{3}}{|\eta|}+W^{I V} D_{0} Y_{0}^{1}+W^{V} D_{0} Y_{0}^{3}+W^{V I} Y_{0}^{1}+W^{V I I} Y_{0}^{3}=0 \\
& D_{0} Y_{1}^{5}+W^{V I I I} Y_{0}^{1}+W^{I X} Y_{0}^{3}=0
\end{aligned}
$$

d'où

$$
D_{0}^{3} Y_{0}^{1}+W D_{0}^{2} Y_{0}^{1}+W^{\prime} D_{0} Y_{0}^{1}+W^{\prime \prime} D_{0} Y_{0}^{3}+W^{\prime \prime \prime} Y_{0}^{1}+W^{I V} Y_{0}^{3}=0
$$

On impose les conditions initiales

$$
Y_{0}\left|=0 ; \quad Y_{1}\right|=I
$$

on obtient

$$
\begin{gathered}
Y_{0}^{1}\left|=Y_{0}^{3}\right|=O ; \quad D_{0} Y_{0}^{1}|=-| \eta\left|I^{2} ; \quad D_{0} Y_{0}^{3}\right|=-|\eta| I^{4} \\
D_{0}^{2} Y_{0}^{1}|=| \eta \mid\left(b_{05}^{2} I^{5}-\hat{\beta}_{1}^{1} I^{2}-\hat{\beta}_{3}^{1} I^{4}\right),
\end{gathered}
$$

d'où tous les coefficients.

ii) ${ }^{\prime \prime} b_{05}^{2}=0, b_{01}^{5} \neq 0$ ou $b_{03}^{5} \neq 0$.

$$
\begin{aligned}
& D_{0} Y_{0}^{5}+b_{01}^{5} Y_{0}^{1}+b_{03}^{5}=0 \\
& D_{0}^{2} Y_{0}^{1}+W^{\prime} D_{0} Y_{0}^{1}+W^{\prime \prime} D_{0} Y_{0}^{3}+W^{\prime \prime \prime} Y_{0}^{1}+W^{I V} Y_{0}^{3}+W^{V} Y_{0}^{5}=0 \\
& D_{0}^{2} Y_{0}^{3}+W^{V I} D_{0} Y_{0}^{1}+W^{V I I} D_{0} Y_{0}^{3}+W^{V I I I} Y_{0}^{1}+W^{I X} Y_{0}^{3}+W^{X} Y_{0}^{5}=0 .
\end{aligned}
$$

On impose les conditions initiales des cas précédents et on détermine tous les coefficients.

\section{§6. Étude du type $(2,1,1,1)$}

$$
h=\left(\begin{array}{ccccc}
D_{0} & \left|D^{\prime}\right| & 0 & 0 & 0 \\
b_{1}^{2} & D_{0} & b_{3}^{2} & b_{4}^{2} & b_{5}^{2} \\
b_{1}^{3} & 0 & D_{0} & b_{4}^{3} & b_{5}^{3} \\
b_{1}^{4} & 0 & b_{3}^{4} & D_{0} & b_{5}^{4} \\
b_{1}^{5} & 0 & b_{3}^{5} & b_{4}^{5} & D_{0}
\end{array}\right)
$$


Les conditions L s'écrivent

$$
\begin{aligned}
(\mathrm{LG})_{2}: b_{01}^{2} \neq & 0 ; \\
(\mathrm{LG})_{3}: b_{01}^{2} \equiv & 0 ; \quad b_{03}^{2} b_{01}^{3}+b_{04}^{2} b_{01}^{4}+b_{05}^{2} b_{01}^{5} \not \equiv 0 ; \\
(\mathrm{LG})_{4}: b_{01}^{2} \equiv & 0 ; \quad b_{03}^{2} b_{01}^{3}+b_{04}^{2} b_{01}^{4}+b_{05}^{2} b_{01}^{5} \equiv 0 ; \\
C_{4} \equiv & b_{03}^{2}\left(b_{04}^{3} b_{01}^{4}+b_{05}^{3} b_{01}^{5}+D_{0} b_{01}^{3}\right)+b_{04}^{2}\left(b_{03}^{4} b_{01}^{3}+b_{05}^{4} b_{01}^{5}+D_{0} b_{01}^{4}\right) \\
& +b_{05}^{2}\left(b_{03}^{5} b_{01}^{3}+b_{04}^{5} b_{01}^{4}+D_{0} b_{01}^{5}\right) \not \equiv 0 ; \\
(\mathrm{LG})_{5}: b_{01}^{2} \equiv & 0 ; \quad b_{03}^{2} b_{01}^{3}+b_{04}^{2} b_{01}^{4}+b_{05}^{2} b_{01}^{5} \equiv 0 ; \quad C_{4} \equiv 0 ;
\end{aligned}
$$

en posant $\beta_{j}^{i}=\sum_{h=1}^{5} b_{0 h}^{i} b_{0 j}^{h}$,

$$
\begin{aligned}
C_{5} \equiv & \beta_{3}^{2} \beta_{1}^{3}+\beta_{4}^{2} \beta_{1}^{4}+\beta_{5}^{2} \beta_{1}^{5}+\beta_{3}^{2} D_{0} b_{01}^{3}+\beta_{4}^{2} D_{0} b_{01}^{4}+\beta_{5}^{2} D_{0} b_{01}^{5} \\
& +b_{03}^{2} D_{0} \beta_{1}^{3}+b_{04}^{2} D_{0} \beta_{1}^{4}+b_{05}^{2} D_{0} \beta_{1}^{5} \\
& +b_{03}^{2} D_{0}^{2} b_{01}^{3}+b_{04}^{2} D_{0}^{2} b_{01}^{4}+b_{05}^{2} D_{0}^{2} b_{01}^{5} \not \equiv 0 \\
\mathrm{~L}: b_{01}^{2} \equiv & 0 ; \quad b_{03}^{2} b_{01}^{3}+b_{04}^{2} b_{01}^{4}+b_{05}^{2} b_{01}^{5} \equiv 0 ; \quad C_{4} \equiv 0 ; \quad C_{5} \equiv 0 .
\end{aligned}
$$

\subsection{Cas $(\mathrm{LG})_{2}$.}

$$
\begin{aligned}
f & =\sum_{1 \leq t \leq 2} f_{1}^{t}+f_{2} ; \\
f_{1}^{t} & =e^{\psi^{t}}\left[Y_{0}^{t}+\cdots Y_{k}^{t}+\cdots\right] ;
\end{aligned}
$$

$\left(D_{0} \psi^{t}\right)^{2}=b_{01}^{2}|\eta| \neq 0 ; \psi^{t}$ est d'ordre $\frac{1}{2}, Y_{k}^{t}$ est d'ordre $\frac{1-k}{2}$;

$$
f_{2}=Z_{0}^{t}+\cdots+Z_{k}^{t}+\cdots \text {, }
$$

$Z_{k}$ est d'ordre $-\frac{k}{2}$.

On obtient, en bref:

(0) $Y_{0}^{2}=0$;

(2) le choix de $D_{0} \psi$ laisse $Y_{0}^{1}$ libre;

(1) $Y_{0}^{3}=Y_{0}^{4}=Y_{0}^{5}=0 ; \quad \ldots$;

(0) $Z_{0}^{2}=0$;

(3) $D_{0} Y_{0}^{1}+Y_{0}^{1}=0$;

(2) $b_{01}^{2} Z_{0}^{1}+b_{03}^{2} Z_{0}^{3}+b_{04}^{2} Z_{0}^{4}+b_{05}^{2} Z_{0}^{5}=0 \quad D_{0} Z_{0}^{3}+b_{01}^{3} Z_{0}^{1}+b_{04}^{3} Z_{0}^{4}+b_{05}^{3} Z_{0}^{5}=0$

$D_{0} Z_{0}^{4}+b_{01}^{4} Z_{0}^{1}+b_{03}^{4} Z_{0}^{4}+b_{05}^{4} Z_{0}^{5}=0 \quad D_{0} Z_{0}^{5}+b_{01}^{5} Z_{0}^{1}+b_{03}^{5} Z_{0}^{4}+b_{04}^{5} Z_{0}^{5}=0$.

On impose les conditions initiales

$$
\sum_{t} Y_{0}^{t}\left|=0 ; \quad \sum_{t} Y_{1}^{t}\right|+Z_{0}=I ; \quad \cdots
$$

on obtient aisément

$$
\begin{gathered}
\sum_{t} Y_{0}^{t 1}\left|=0 ; \quad \sum_{t} D_{0} \psi^{t} Y_{0}^{t 1}\right|=|\eta| I^{2} \\
Z_{0}^{3}\left|=I^{3} ; \quad Z_{0}^{4}\right|=I^{4} ; \quad Z_{0}^{5} \mid=I^{5}
\end{gathered}
$$

d'où tous les coefficients. 
6.2. Cas $(\mathrm{LG})_{3}$.

$$
\begin{aligned}
f & =\sum_{1 \leq t \leq 3} f_{1}^{t}+f_{2} \\
f_{1}^{t} & =e^{\psi^{t}}\left[Y_{0}^{t}+\cdots Y_{k}^{t}+\cdots\right]
\end{aligned}
$$

$\left(D_{0} \psi^{t}\right)^{3}=-\left(b_{03}^{2} b_{01}^{3}+b_{04}^{2} b_{01}^{4}+b_{05}^{2} b_{01}^{5}\right)|\eta| \neq 0 ; \psi^{t}$ est d'ordre $\frac{1}{3}, Y_{k}^{t}$ est d'ordre $\frac{2-k}{3} ;$

$$
f_{2}=Z_{0}^{t}+\cdots+Z_{k}^{t}+\cdots
$$

$Z_{k}$ est d'ordre $-\frac{k}{3}$.

On obtient, en bref:

(0) $Y_{0}^{2}=0 ; \quad \ldots ; \quad$ (2) $Y_{0}^{3}=Y_{0}^{4}=Y_{0}^{5}=0 \quad \ldots$;

(4) le choix de $D_{0} \psi$ laisse $Y_{0}^{1}$ libre; (5) $D_{0} Y_{0}^{1}+W Y_{0}^{1}=0$;

(0) $Z_{0}^{2}=0 ; \quad$ (3) on obtient aisément:

$$
\begin{aligned}
& b_{03}^{2} Z_{0}^{3}+b_{04}^{2} Z_{0}^{4}+b_{05}^{2} Z_{0}^{5}=0 \\
& \left(b_{03}^{2} b_{01}^{3}+b_{04}^{2} b_{01}^{4}+b_{05}^{2} b_{01}^{5}\right) Z_{0}^{1}+\left(b_{04}^{2} b_{03}^{4}+b_{05}^{2} b_{03}^{5}-D_{0} b_{03}^{2}\right) Z_{0}^{3} \\
& \quad+\left(b_{03}^{2} b_{04}^{3}+b_{05}^{2} b_{04}^{5}-D_{0} b_{04}^{2}\right) Z_{0}^{4}+\left(b_{03}^{2} b_{05}^{3}+b_{04}^{2} b_{05}^{4}-D_{0} b_{05}^{2}\right) Z_{0}^{5}=0 \\
& D_{0} Z_{0}^{3}+b_{01}^{3} Z_{0}^{1}+b_{04}^{3} Z_{0}^{4}+b_{05}^{3} Z_{0}^{5}=0 \quad D_{0} Z_{0}^{4}+b_{01}^{4} Z_{0}^{1}+b_{03}^{4} Z_{0}^{3}+b_{05}^{4} Z_{0}^{5}=0 \\
& D_{0} Z_{0}^{5}+b_{01}^{5} Z_{0}^{1}+b_{03}^{5} Z_{0}^{3}+b_{04}^{5} Z_{0}^{4}=0 .
\end{aligned}
$$

$Z_{0}^{1}$ s'exprime à l'aide de $Z_{0}^{3}, Z_{0}^{4}, Z_{0}^{5}$, et on obtient selon les cas deux équations différentielles, par exemple, si $b_{03}^{2} \neq 0$ :

$$
\begin{array}{r}
D_{0} Z_{0}^{4}+W Z_{0}^{4}+W^{\prime} Z_{0}^{5}=0 \\
D_{0} Z_{0}^{5}+W^{\prime \prime} Z_{0}^{4}+W^{\prime \prime \prime} Z_{0}^{5}=0
\end{array}
$$

On impose les conditions initiales

$$
\sum_{t} Y_{0}^{t}\left|=\sum_{t} Y_{1}^{t}\right|=0 ; \quad \sum_{t} Y_{2}^{t} \mid+Z_{0}=I ; \quad \cdots
$$

on détermine les données initiales et on obtient tous les coefficients.

6.3. Cas $(\mathrm{LG})_{4}$.

$$
\begin{aligned}
f & =\sum_{1 \leq t \leq 4} f_{1}^{t}+f_{2} \\
f_{1}^{t} & =e^{\psi^{t}}\left[Y_{0}^{t}+\cdots Y_{k}^{t}+\cdots\right]
\end{aligned}
$$


$\left(D_{0} \psi^{t}\right)^{4}=C_{4}|\eta| ; \psi^{t}$ est d'ordre $\frac{1}{4}, Y_{k}^{t}$ est d'ordre $\frac{3-k}{4}$

$$
f_{2}=Z_{0}^{t}+\cdots+Z_{k}+\cdots
$$

$Z_{k}$ est d'ordre $-\frac{k}{4}$.

On obtient:

(0) $Y_{0}^{2}=0 ; \quad \ldots ; \quad$ (3) $Y_{0}^{3}=Y_{0}^{4}=Y_{0}^{5}=0 \quad \ldots$;

(6) le choix de $D_{0} \psi$ laisse $Y_{0}^{1}$ libre; (7) $D_{0} Y_{0}^{1}+W Y_{0}^{1}=0$;

(0) $Z_{0}^{2}=0 ; \quad \ldots$;

(4) $b_{03}^{2} Z_{0}^{3}+b_{04}^{2} Z_{0}^{4}+b_{05}^{2} Z_{0}^{5}=0$

$$
\begin{gathered}
\left(b_{04}^{2} b_{03}^{4}+b_{05}^{2} b_{03}^{5}-D_{0} b_{03}^{2}\right) Z_{0}^{3}+\left(b_{03}^{2} b_{04}^{3}+b_{05}^{2} b_{04}^{5}-D_{0} b_{04}^{2}\right) Z_{0}^{4} \\
+\left(b_{03}^{2} b_{05}^{3}+b_{04}^{2} b_{05}^{4}-D_{0} b_{05}^{2}\right) Z_{0}^{5}=0 \\
{\left[\left(b_{04}^{2} b_{03}^{4}+b_{05}^{2} b_{03}^{5}-D_{0} b_{03}^{2}\right) b_{01}^{3}+\left(b_{03}^{2} b_{04}^{3}+b_{05}^{2} b_{04}^{5}-D_{0} b_{04}^{2}\right) b_{01}^{4}\right.} \\
\left.\quad+\left(b_{03}^{2} b_{05}^{3}+b_{04}^{2} b_{05}^{4}-D_{0} b_{05}^{2}\right) b_{01}^{5}\right] Z_{0}^{1} \\
+\left[\left(b_{03}^{2} b_{04}^{3}+b_{05}^{2} b_{04}^{5}-D_{0} b_{04}^{2}\right) b_{03}^{4}+\left(b_{03}^{2} b_{05}^{3}+b_{04}^{2} b_{05}^{4}-D_{0} b_{05}^{2}\right) b_{03}^{5}\right. \\
\left.\quad-D_{0}\left(b_{04}^{2} b_{03}^{4}+b_{05}^{2} b_{03}^{5}-D_{0} b_{03}^{2}\right)\right] Z_{0}^{3} \\
+[\ldots] Z_{0}^{4}+[\ldots] Z_{0}^{5}=0
\end{gathered}
$$

les coefficients de $Z_{0}^{4}$ et $Z_{0}^{5}$ se déduisent par une permutation facile; $Z_{0}^{1}$ s'exprime à l'aide de $Z_{0}^{3}, Z_{0}^{4}, Z_{0}^{5}$. On a aussi les équations $D_{0} Z_{0}^{3}+\cdots=0, D_{0} Z_{0}^{4}+\cdots=0$, $D_{0} Z_{0}^{5}+\cdots=0$, du cas $(\mathrm{LG})_{3}$.

On doit distinguer plusieurs cas; on obtient, par exemple:

$$
D_{0} Z_{0}^{5}+W Z_{0}^{5}=0 \text {. }
$$

On impose les conditions initiales

$$
\sum_{t} Y_{0}^{t}\left|=\sum_{t} Y_{1}^{t}\right|=\sum_{t} Y_{2}^{t}\left|=0 ; \quad \sum_{t} Y_{3}^{t}\right|+Z_{0}=I ; \quad \cdots
$$

on détermine les données initiales et on obtient tous les coefficients.

6.4. Cas $(\mathbf{L G})_{5}$.

$$
\begin{aligned}
f & =\sum_{1 \leq t \leq 5} f_{1}^{t} \\
f^{t} & =e^{\psi^{t}}\left[Y_{0}^{t}+\cdots Y_{k}^{t}+\cdots\right]
\end{aligned}
$$

$\left(D_{0} \psi^{t}\right)^{5}=C_{5}|\eta| \neq 0 ; \psi^{t}$ est d'ordre $\frac{1}{5}, Y_{k}^{t}$ est d'ordre $\frac{4-k}{5} ;$ 
On obtient:

(2) $Y_{0}^{2}=0 ; \quad \ldots ;$

(4) $Y_{0}^{3}=Y_{0}^{4}=Y_{0}^{5}=0 ; \quad \ldots$;

(8) le choix de $D_{0} \psi$ laisse $Y_{0}^{1}$ libre;

(9) $D_{0} Y_{0}^{1}+W Y_{0}^{1}=0$.

On impose les conditions initiales

$$
\sum_{t} Y_{0}^{t}\left|=\sum_{t} Y_{1}^{t}\right|=\sum_{t} Y_{2}^{t}\left|=\sum_{t} Y_{3}^{t}\right|=0 ; \quad \sum_{t} Y_{4}^{t} \mid=I ; \quad \cdots
$$

on détermine les données initiales et on obtient tous les coefficients.

\subsection{Cas L.}

$$
f_{1}=Y_{0}^{t}+\cdots Y_{k}^{t}+\cdots
$$

$Y_{k}^{t}$ est d'ordre $1-k ;$ On a

$$
\text { (0) } Y_{0}^{2}=0 \text {. }
$$

On distingue des cas en définissant:

$$
M=\left(\begin{array}{ccc}
b_{03}^{2} & \cdots & \cdots \\
b_{04}^{2} b_{03}^{4}+b_{05}^{2} b_{03}^{5}-D_{0} b_{03}^{2} & \cdots & \cdots \\
\left(\begin{array}{l}
b_{04}^{2} b_{05}^{4} b_{03}^{5}+b_{05}^{2} b_{04}^{5} b_{03}^{4}-2 D_{0} b_{04}^{2} b_{03}^{4}-2 D_{0} b_{05}^{2} b_{03}^{5} \\
+D_{0}^{2} b_{03}^{2}-b_{04}^{2} D_{0} b_{03}^{4}-b_{05}^{2} D_{0} b_{03}^{5}+b_{03}^{2} b_{04}^{3} b_{03}^{4}+b_{03}^{2} b_{05}^{3} b_{03}^{5}
\end{array}\right) & \cdots & \cdots
\end{array}\right)
$$

les 2 autres colonnes s'obtiennent par permutation circulaire en $3,4,5$.

a) dét $M \neq 0$. Dans ce cas on a donc: $b_{01}^{3}=b_{01}^{4}=b_{01}^{5}=0$; aucune ligne de $M$ n'est identiquement nulle.

L'étape (1) implique $Y_{0}^{3}=Y_{0}^{4}=Y_{0}^{5}=0$, compte tenu de l'hypothèse de ce cas et des conditions $\mathrm{L}$.

L'étape (2) donne:

$$
D_{0}^{5} Y_{0}^{1}+W D_{0}^{4} Y_{0}^{1}+W^{\prime} D_{0}^{3} Y_{0}^{1}+W^{\prime \prime} D_{0}^{2} Y_{0}^{1}+W^{\prime \prime \prime} D_{0} Y_{0}^{1}+W^{I V} Y_{0}^{1}=0 .
$$

On impose les conditions initiales:

$$
Y_{0}\left|=0 ; \quad Y_{1}\right|=I ; \quad \cdots
$$

on obtient

$$
\begin{aligned}
& \text { (0) } Y_{0}^{1} \mid=0 ; \quad \text { (1) } \quad D_{0} Y_{0}^{1}|=| \eta\left|I^{2} ; \quad D_{0}^{2} Y_{0}^{1}\right|=|\eta| \sum_{C} M_{C}^{1} I^{C}, \quad 3 \leq C \leq 5 \\
& D_{0}^{3} Y_{0}^{1}|=-| \eta\left|\sum_{C} M_{C}^{2} I^{C} ; \quad D_{0}^{4} Y_{0}^{1}\right|=|\eta| \sum_{C} M_{C}^{3} I^{C} .
\end{aligned}
$$


b) dét $M=0$ et un mineur d'ordre 2 est différente de 0 . On obtient, de même, par exemple

$$
\begin{aligned}
& D_{0} Y_{0}^{5}+W Y_{0}^{1}+W^{\prime} Y_{0}^{5}=0 \\
& D_{0}^{4} Y_{0}^{1}+W^{\prime \prime} D_{0}^{3} Y_{0}^{1}+W^{\prime \prime \prime} D_{0}^{2} Y_{0}^{1}+W^{I V} D_{0} Y_{0}^{1}+W^{V} Y_{0}^{1}=0
\end{aligned}
$$

On impose les conditions initiales et on détermine tous les coefficients.

c) Tous les mineur d'ordre 2 sont nuls. La $1^{0}$ ligne n'est pas nulle. On obtient, par exemple:

$$
\begin{aligned}
& D_{0} Y_{0}^{4}+W^{\prime} Y_{0}^{1}+W^{\prime \prime} Y_{0}^{4}+W^{\prime \prime \prime} Y_{0}^{5}=0 \\
& D_{0} Y_{0}^{5}+W^{I V} Y_{0}^{1}+W^{V} Y_{0}^{4}+W^{V I} Y_{0}^{5}=0 \\
& D_{0}^{3} Y_{0}^{1}+W^{V I I} D_{0}^{2} Y_{0}^{1}+W^{V I I I} D_{0} Y_{0}^{1}+W^{I X} Y_{0}^{1}+W^{X} Y_{0}^{4}+W^{X I} Y_{0}^{5}=0 .
\end{aligned}
$$

On impose les conditions initiales et on détermine tous les coefficients.

d) $b_{03}^{2}=b_{04}^{2}=b_{05}^{2}=0$. On obtient

$$
\begin{aligned}
& D_{0} Y_{0}^{3}+b_{01}^{3} Y_{0}^{1}+b_{04}^{3} Y_{0}^{4}+b_{05}^{3} Y_{0}^{5}=0 \\
& D_{0} Y_{0}^{4}+b_{01}^{4} Y_{0}^{4}+b_{03}^{4} Y_{0}^{3}+b_{05}^{4} Y_{0}^{5}=0 \\
& D_{0} Y_{0}^{5}+b_{01}^{5} Y_{0}^{1}+b_{03}^{5} Y_{0}^{3}+b_{04}^{5} Y_{0}^{4}=0 \\
& D_{0}^{2} Y_{0}^{1}+W D_{0} Y_{0}^{1}+W^{\prime} Y_{0}^{1}+W^{\prime \prime} Y_{0}^{3}+W^{\prime \prime \prime} Y_{0}^{4}+W^{I V} Y_{0}^{5}=0 .
\end{aligned}
$$

On impose les conditions initiales et on détermine tous les coefficients.

\section{§7. Étude du type $(1,1,1,1,1)$}

Le diagramme est vide. Le système est, en fait, fortement hyperbolique, comme le montre une démonstration directe, sans réduction, dans [1].

Il n'y a pas de conditions sur les termes d'ordre inférieur.

$\mathrm{Si}$ on réduit le système, on obtient:

$$
h=I D_{0}+b
$$

$Y=Y_{0}$ est d'ordre $0, D_{0} Y+b_{0} Y=0,\left.Y\right|_{x_{0}=\underline{x_{0}}}=I$; d'où $Y$ est la paramétrixe $F$.

\section{§8. Applications}

8.1. Dans tous les cas de multiplicité $\leq 5$, on a le:

ThÉORÈme 8.1. Une condition nécessaire et suffisante pour que le problème de Cauchy soit localement bien posé en $\mathcal{C}^{\infty}$ est que les conditions L soient satisfaites. 


\subsection{Résumé de la preuve de la condition nécessaire $[14,4,22]$.}

a) Pour un type donné, on peut définir une relation d'ordre entre les conditions $\mathrm{L}$ en suivant l'ordre de l'établissement des conditions $(\mathrm{LG})_{d}$. Par exemple, pour le type $(3,2) \S 3$, on les ordonne dans l'ordre (sous la forme réduite): $b_{01}^{3}=0$, $b_{04}^{3} b_{01}^{5}=0$, si $b_{04}^{3} \not \equiv 0,\left[b_{02}^{3}\right]^{2}+b_{04}^{3} b_{01}^{4}=0, b_{02}^{3}+b_{04}^{5}=0, C^{\prime}=0, C_{3}^{\prime}=0, C_{4}^{\prime}=0$, $C_{5}^{\prime}=0$; de même si $b_{01}^{5} \neq 0$, on a l'ordre indiqué dans le paragraphe $\S 3$; de même si $b_{04}^{3}=b_{01}^{5}=0$, on a l'ordre indiqué dans ce paragraphe.

Pour démontrer la nécessité d'une condition $\mathrm{L}_{i j}$, on supposera donc les conditions précédant $\mathrm{L}_{i j}$ vérifiées.

b) D'autre part, si le problème de Cauchy est bien posé en $\underline{x}$, on déduit du théorème du graphe fermé, qu'il existe un voisinage de $\underline{x}$, un entier $\tau$, un compact $\mathcal{K}$ du voisinage, une constante $C>0$ tels que, pour tout $u \in \mathcal{C}^{\infty}$, on ait

$$
|u|_{0, \mathcal{K}} \leq C\left\{|h u|_{\tau, \mathcal{K}}+\left|u\left(\underline{x_{0}}, x^{\prime}\right)\right|_{\tau, \mathcal{K} \cap\left\{x_{0}=\underline{x_{0}}\right.}\right\},
$$

où $|u|_{\tau, \mathcal{K}}$ désigne une semi-norme usuelle de $\mathcal{C}^{\infty}$ (la borne supérieure des dérivées jusqu'à l'ordre $\tau$ sur le compact $\mathcal{K}$ ).

On remarque aussi que les conditions $\mathrm{L}$ expriment l'annulation de fonctions continues. Il suffit donc, de démontrer que si la condition $\mathrm{L}_{j k}$ considérée n'est pas vérifiée, dans un ensemble partout dense, on peut trouver $u$ qui viole l'inégalité du graphe fermé.

c) Comme $\mathrm{L}_{j k}$ n'est pas vérifiée, il résulte facilement des calculs précédents, que l'on peut trouver, pour tout $k_{0}^{\prime}>0$ un développement

$$
u_{k_{0}}=\exp \left(i \omega \delta \varphi_{j}+\cdots+\omega^{1 / d} \psi_{1 j}\right)\left(\sum_{0 \leq k \leq k_{0}} Y_{k}(x) \omega^{-k / d^{\prime}}\right)
$$

tels que pour $k_{0}$ assez grand

$$
\left|h u_{k_{0}}\right|_{\tau, \mathcal{K}}=O\left(\omega^{-k_{0}^{\prime}}\right) \exp \left[\sup _{\mathcal{K}}\left(\operatorname{Re} \psi_{1 j}(x)\right) \omega^{1 / d}\right]
$$

$k_{0}^{\prime}>0, \omega$ tend vers $+\infty$.

Comme dans le $\S 0.5$, on a

$$
H\left(x, D_{0} \varphi_{j}, D^{\prime} \varphi_{j}\right)=0, \quad \varphi_{j}\left(\underline{x_{0}}, x^{\prime}, \eta^{\prime}\right)=x^{\prime} \cdot \eta^{\prime}
$$

( $\varphi_{j}$ est évidemment à valeurs réelles) et en utilisant les paragraphes suivants, on a choisi la phase $\psi_{1 j}$ de sorte que: $D_{0}\left(\operatorname{Re} \psi_{1 j}\right)>0,(\delta= \pm 1$, comme dans [1] est utile pour éviter le cas $\left(D_{0} \psi_{1}\right)^{2}=$ un réel $\left.<0\right)$. 
On a, donc, de plus:

$$
\left|u_{k_{0}, 0}\right|_{0, \mathcal{K}}=\exp \left[\sup _{\mathcal{K}}\left(\operatorname{Re} \psi_{1 j}(x)\right) \omega^{1 / d}\right](U+o(\omega)), \quad U \neq 0
$$

par le choix de $Y_{0}(x) \neq 0$ dans le voisinage considéré, et

$$
\left|u_{0}\left(\underline{x_{0}}, x^{\prime}\right)\right|_{\tau, \mathcal{K} \cap\left\{x_{0}=\underline{x_{0}}\right\}}=O\left(\omega^{\tau}\right) \exp \left[\sup _{\mathcal{K}}\left(\operatorname{Re} \psi_{1 j}\left(\underline{x_{0}}, x^{\prime}\right)\right) \omega^{1 / d}\right](U+o(\omega)) .
$$

Pour $\omega$ assez grand, on obtient une fonction $u_{k_{0}}$ qui viole l'inégalité du graphe fermé.

\subsection{Résumé de la preuve de la condition suffisante.}

a) Dans [12] Matsumoto et Yamahara construisent une solution fondamentale analytique pour l'opérateur $h$.

b) Pour les différents types, lorsque les conditions L sont vérifiées, on a construit, en dehors de l'ensemble $\Sigma$ du $\S 0.4$ des paramétrixes $F$ qui donnent un expression, modulo des opérateurs analytiques d'ordre $-\infty$, de la solution fondamentale.

En passant, comme usuellement, au complexe, les inégalité satisfaites par les coefficients $Y$ de ces paramétrixes, en dehors de $\Sigma$, se prolongent dans chaque voisinage conique [11], et on a construit une paramétrixe $F$ qui opère bien sur les fonctions $\mathcal{C}^{\infty}$ dans $x_{0}=x_{0}$ et est à valeurs dans les fonctions $\mathcal{C}^{\infty}$. On obtient alors aisément l'existence de la solution du problème de Cauchy $\mathcal{C}^{\infty}$. Comme l'opérateur transposé vérifie aussi les conditions $\mathrm{L}$, on a comme usuellement, l'unicité de la solution.

\section{4.}

ThÉORÈme 8.2. Le problème de Cauchy est localement bien posé dans la classe $\gamma^{d^{\prime}}, 1<d^{\prime} \leq d=\frac{p}{p-1}$, pour $m_{1} \leq 5$.

THÉORÈme 8.3. Les conditions sont définie au $§ 0.2 \S 0.3$.

On donne les conditions suffisantes pour que le problème de Cauchy soit localement bien posé dans $\gamma^{d^{\prime}}, 1<d^{\prime} \leq d$.

1. Type (5);
(a) Si $\mathrm{L}_{11}$ est vérifiée, $d=\frac{4}{3}$.
(b) $S i \mathrm{~L}_{12}$ est vérifiée, $d=\frac{3}{2}$.
(c) Si $\mathrm{L}_{13}$ est vérifiée, $d=\frac{5}{3}$.
(d) Si $\mathrm{L}_{13}$ et $\mathrm{L}_{(2) 3}$ sont vérifiées, $d=2$.
(e) $S i \mathrm{~L}_{1}, \mathrm{~L}_{23}$ sont vérifiées, $d=\frac{5}{2}$.
(f) $S i \mathrm{~L}_{1}, \mathrm{~L}_{23}$ et $\mathrm{L}_{(3) 4}$ sont vérifiées, $d=3$. 
(g) Si $\mathrm{L}_{1}, \mathrm{~L}_{2}$ et $\mathrm{L}_{33}$ sont vérifiées, $d=4$.

(h) Si $\mathrm{L}_{1}, \mathrm{~L}_{2}$ et $\mathrm{L}_{3}$ sont vérifiées, $d=5$.

(i) Si les conditions $\mathrm{L}$ sont vérifiées, $d$ est quelconque.

2. Type $(4,1)$;
(a) Si $\mathrm{L}_{11}$ est vérifiée, $d=\frac{3}{2}$.
(b) $S i \mathrm{~L}_{12}$ est vérifiée, $d=\frac{5}{3}$.
(c) Si $\left(\mathrm{L}_{12}\right.$ et $\left.\mathrm{L}_{(2) 2}\right)$ ou $\left(\mathrm{L}_{1}\right.$ et $\left.\mathrm{L}_{21}\right)$ sont vérifiées, $d=2$.
(d) Si $\mathrm{L}_{1}$ et $\mathrm{L}_{22}$ sont vérifiées, $d=\frac{5}{2}$.
(e) Si $\mathrm{L}_{1}, \mathrm{~L}_{22}$ et $\mathrm{L}_{(3) 3}$ sont vérifiées, $d=3$.
(f) $S i \mathrm{~L}_{1}, \mathrm{~L}_{2}$ et $\mathrm{L}_{32}$ sont vérifiées, $d=4$.
(g) Si $\mathrm{L}_{1}, \mathrm{~L}_{2}$ et $\mathrm{L}_{3}$ sont vérifiées, $d=5$.
(h) Si les conditions $\mathrm{L}$ sont vérifiées, d est quelconque.

3. Type $(3,2)$;
(a) Si $\mathrm{L}_{1}$ est vérifiée, $d=\frac{5}{3}$.
(b) $S i \mathrm{~L}_{1}$ et $\mathrm{L}_{21}$ sont vérifiées, $d=2$.
(c) Si $\mathrm{L}_{1}$ et $\mathrm{L}_{2}$ sont vérifiées et si $\mathrm{L}_{12}$ ne l'est pas, $d=\frac{5}{2}$.
(d) Si ( $\mathrm{L}_{12}$ n'est pas vérifiée et si $\mathrm{L}_{1}, \mathrm{~L}_{2}$ et $\mathrm{L}_{(4) 2}$ sont vérifiées) ou $\left(\mathrm{L}_{12}\right.$ et $\mathrm{L}_{3}$ sont vérifiées), $d=3$.
(e) $S i\left(\mathrm{~L}_{12}\right.$ n'est pas vérifiée $\mathrm{L}_{1}, \mathrm{~L}_{2}$ et $\mathrm{L}_{3}$ sont vérifiées) ou $\left(\mathrm{L}_{12}, \mathrm{~L}_{3}\right.$ et $\mathrm{L}_{4}$ sont vérifiées), $d=4$.
(f) Si $\left(\mathrm{L}_{12}\right.$ n'est pas vérifiée $\mathrm{L}_{1}, \mathrm{~L}_{2}, \mathrm{~L}_{3}$ et $\mathrm{L}_{4}$ sont vérifiées) ou $\left(\mathrm{L}_{12}, \mathrm{~L}_{3}, \mathrm{~L}_{4}\right.$ et $\mathrm{L}_{6}$ sont vérifiées), $d=5$.
(g) Si les conditions $\mathrm{L}$ sont vérifiées, $d$ est quelconque.

4. Type $(3,1,1)$;
(a) Si $\mathrm{L}_{11}$ est vérifiée, $d=2$.
(b) Si $\mathrm{L}_{1}$ et $\mathrm{L}_{21}$ sont vérifiées, $d=\frac{5}{2}$.
(c) Si $\mathrm{L}_{1}, \mathrm{~L}_{21}$ et $\mathrm{L}_{(3) 2}$ sont vérifiées, $d=3$.
(d) $S i \mathrm{~L}_{1}, \mathrm{~L}_{2}$ et $\mathrm{L}_{31}$ sont vérifiées, $d=4$.
(e) $S i \mathrm{~L}_{1}, \mathrm{~L}_{2}$ et $\mathrm{L}_{3}$ sont vérifiées, $d=5$.
(f) Si les conditions $\mathrm{L}$ sont vérifiées, $d$ est quelconque.

5. Type $(2,2,1)$;

(a) Si $\mathrm{L}_{2}$ est vérifiée et $\mathrm{L}_{11}$ ne l'est pas, $d=\frac{5}{2}$. 
(b) Si $\left(\mathrm{L}_{2}\right.$ et $\left.\mathrm{L}_{(4) 1}\right)$ ou $\mathrm{L}_{11}$ sont vérifiées, $d=3$.

(c) Si $\left(\mathrm{L}_{11}\right.$ n'est pas vérifiée, $\mathrm{L}_{2}$ et $\mathrm{L}_{3}$ sont vérifiées $)$ ou $\left(\mathrm{L}_{11}\right.$ et $\mathrm{L}_{4}$ sont vérifiées), $d=4$.

(d) Si ( $\mathrm{L}_{11}$ n'est pas vérifiée, $\mathrm{L}_{2}, \mathrm{~L}_{3}$ et $\mathrm{L}_{4}$ sont vérifiées) ou $\left(\mathrm{L}_{11}, \mathrm{~L}_{4}\right.$ et $\mathrm{L}_{5}$ sont vérifiées), $d=5$.

(e) Si les conditions $\mathrm{L}$ sont vérifiées, $d$ est quelconque.

6. Type $(2,1,1,1)$;

(a) $S i \mathrm{~L}_{1}$ est vérifiée, $d=3$.

(b) $S i \mathrm{~L}_{1}$ et $\mathrm{L}_{2}$ sont vérifiées, $d=4$.

(c) $S i \mathrm{~L}_{1}, \mathrm{~L}_{2}$ et $\mathrm{L}_{3}$ sont vérifiées, $d=5$.

(d) Si les conditions $\mathrm{L}$ sont vérifiées, $d$ est quelconque.

7. Type $(1,1,1,1,1)$; le système est fortement hyperbolique; $d$ est quelconque.

8.5. Résumé de la preuve des théorèmes 8.2 et 8.3. Elle est semblable à celle du $§ 8.3$. Dans chaque cas, les paramétrixes $F_{j}$ d'ordre infini [19, pp. 124, 180] ont des amplitudes $f_{j}$ analytiques, dont le terme principal est de la forme $\S 0.5$ :

$$
e^{\psi\left(x, \eta^{\prime}\right)} Y\left(x, \eta^{\prime}\right)
$$

le terme principal de la phase est d'ordre $\frac{1}{d}$ en $\eta^{\prime}$; on en déduit des majorations de l'exponentielle où apparaît:

$$
\exp \left(B\left|\eta^{\prime}\right|^{\frac{1}{d}}\right)
$$

La paramétrixe opère alors sur les fonctions de $\gamma^{d}$ dans $x_{0}=\underline{x_{0}}$ et est à valeurs dans les fonctions de $\gamma^{d}$. On en déduit le théorème d'existence et d'unicité dans $\gamma^{d}$.

\section{Références}

[ 1 ] R. Berzin and J. Vaillant, Systèmes hyperboliques à caractéristiques multiples, J. Math. Pures Appl., 58-2 (1979), 165-216.

[2] L.B. de Monvel and P. Kree, Pseudo-differential operators and Gevrey classes, Ann. Inst. Fourier (Grenoble), 17-1 (1967), 295-323.

[ 3 ] J. Chazarain, Propagation des singularités pour une classe d'operateurs à caractéristiques multiples et résolubilite locale, Ann. Inst. Fourier (Grenoble), 24-1 (1974), 203-223.

[ 4 ] H. Flaschka and G. Strang, The correctness of the Cauchy problem, Adv. in Math., 6 (1971), 347-379.

[ 5 ] Y. Hamada, J. Leray and C. Wagschal, Systèmes d'équations aux dérivée partielles à caractéristiques multiples: problème de Cauchy ramifié; hyperbolicité partielle, J. Math. Pures Appl., 55-3 (1976), 297-352. 
[ 6 ] K. Kajitani, Cauchy problem for non strictly hyperbolic systems, Publ. Res. Inst. Math. Sci., 15-2 (1979), 519-550.

[ 7 ] K. Kajitani, Leray-Volevich systems and Gevrey class, J. Math. Kyoto Univ., 21-3 (1981), 547-574.

[ 8 ] K. Kajitani and T. Nishitani, The hyperbolic Cauchy problem, Lecture Notes in Math., vol. 1505, Springer-Verlag, Berlin, 1991.

[ 9 ] H. Komatsu, Irregularity of hyperbolic operators, Hyperbolic equations and related topics (Katata/Kyoto, 1984), Academic Press, Boston, MA, 1986, pp. 155-179.

[10] J. Leray and Y. Ohya, Systèmes linéaires, hyperboliques non-stricts, Hyperbolic equations and waves (Rencontres, Battelle Res. Inst., Seattle, Wash., 1968), Springer-Verlag Berlin-Heidelberg-New York, 1970, pp. 283-322.

[11] W. Matsumoto, Normal form of systems of partial differential and pseudo-differential operators in formal symbol classes and applications, J. Math. Kyoto Univ., 34-1 (1994), 15-40.

[12] W. Matsumoto and H. Yamahara, On the Cauchy-Kowaleskaya theorem for systems, Proc. Japan Acad., 67-6 (1991), 181-185.

[13] M. Miyake, On Cauchy-Kowalevsky's theorem for general systems, Publ. Res. Inst. Math. Sci., 15-2 (1979), 315-337.

[14] S. Mizohata, On the Levi condition, Calcul d'opérateurs et fronts d'ondes (Jean Vaillant, ed.), no. 29, Hermann, Paris, 1988, pp. 39-64.

[15] T. Nishitani, On the Lax-Mizohata theorem in the analytic and Gevrey classes, J. Math. Kyoto Univ., 18-3 (1978), 509-521.

[16] Y. Ohya, Le problème de Cauchy pour les équations hyperboliques à caractéristiques multipes, Math. Soc. Japan, 16 (1964), 268-296.

[17] V.M. Petkov, Microlocal forms for hyperbolic systems, Math. Nachr., 93 (1979), 117-131.

[18] V.M. Petkov, The parametrix of the Cauchy problem for nonsymmetrisable hyperbolic systems with characteristics of constant multiplicity, Trans. Moscow Math. Soc., 37-1 (1980), 1-47.

[19] L. Rodino, Linear partial differential operators in Gevrey spaces, World Scientific Publishing Co., Inc., River Edge, NJ, 1993.

[20] G. Taglialatela and Jean Vaillant, Conditions invariantes d'hyperbolicité des systèmes et réduction des systèmes, Bull. Sci. Math., 120 (1996), 19-97.

[21] J. Vaillant, Données de Cauchy portées par une caractéristique double, dans le cas d'un système linéaire d'équations aux derivées partielles, rôle des bicaractéristiques, J. Math. Pures Appl., 47 (1968), 1-40.

[22] J. Vaillant, Conditions d'hyperbolicité des systèmes d'opérateurs aux dérivées partielles, Bull. Sci. Math., 114 (1990), 243-328.

[23] J. Vaillant, Conditions d'hyperbolicité des systèmes, C. R. Acad. Sci. Paris, 313-5 (1991), 227-230.

[24] J. Vaillant, Systèmes d'équations aux dérivées partielles et classes de Gevrey, C. R. Acad. Sci. Paris. 320-5 (1995), 1469-1474.

[25] J. Vaillant, Analytic hyperbolic systems, Workshop on General Theory of Partial Differential Equations and Microlocal Analysis (Luigi Rodino, ed.), Pitnam Research Notes, 1996, 4-15 September 1995, Trieste, pp. 209-229.

[26] J. Vaillant, Systèmes différentiels à multiplicité constante: un exemple, - (Jaime Carvalho e Silva and Jean Vaillant, eds.), Texto matematica (à paraître).

[27] J. Vaillant, Diagonalisation et décomposition d'un système, en preparation. 
UFR 920 - UMR 7586

MATHÉMATHIQUES, B.C.172

TOUR 46-0, $5^{\text {ĖME }}$ ÉTAGE

UNIVERSITÉ PARIS VI

4, Place Jussieu

75252 PARIS CEDEX 05

FRANCE 